\title{
STRUCTURAL CHARACTERIZATION OF SECONDARY METABOLITES PRODUCED BY FUNGI OBTAINED FROM DAMP CANADIAN BUILDINGS
}

\section{David Roderick McMullin}

B.Sc. Carleton University, 2008

\begin{abstract}
A thesis submitted to the Faculty of Graduate Studies and Research in partial fulfillment of the requirements for the degree of
\end{abstract}

\section{DOCTOR OF PHILOSOPHY}

\section{Chemistry with a Specialization in Chemical and Environmental Toxicology}

\author{
Ottawa-Carleton Institute of Chemistry \\ Carleton University \\ Ottawa, ON, Canada
}

(C) Copyright

2014. David R. McMullin 


\begin{abstract}
A comprehensive investigation of the secondary metabolites produced by Chaetomium globosum, Wallemia sebi, Penicillium corylophilum and four Trichoderma species obtained from Canadian buildings is presented. Atopic and non-atopic individuals occupying damp, moldy buildings are at increased risk of both allergic and non-allergic adverse health effects. There is now strong toxicological evidence showing that secondary metabolites, including mold specific glucan, present on spores and mycelial fragments are in part responsible for these effects. At the low doses that could be experienced by the human lung indoors, metabolites from fungi have been demonstrated to alter the expression of genes involved with asthma in vivo and in vitro. These genetic alterations are accompanied by histological disruptions and inflammatory responses.
\end{abstract}

The primary focus of this study was to identify and isolate the dominant toxins produced by the mentioned fungi obtained from Canadian buildings. Isolates were grown in liquid culture and screened for metabolite production. Metabolites were purified by various chromatographic methods and their structures were unambiguously determined by mass spectrometry and detailed analysis of spectroscopic data.

In this work, $C$. globosum primarily produced chaetoglobosin $\mathrm{A}, \mathrm{C}$ and $\mathrm{F}$, chaetomugilin $\mathrm{D}$ and chaetoviridin A. Other chaetoglobosins and azaphilones were produced in minor amounts including three new azaphilones. The meroterpenoids, andrastin A and citreohybridinol, koninginins A, E and G, the eremophilane sesquiterpene phomenone and seven new metabolites, four isochromans and three $\alpha$-pyrones, were isolated from $P$. corylophilum. This was the first 
report of meroterpenoids and $\alpha$-pyrones from this species as well as koninginins from any genus outside of Trichoderma. The xerophile $W$. sebi produced walleminone, a new alkaloid, wallimidione, tryptophol and other amino acid derived compounds. T. citrinoviride produced many sorbicillin derived compounds including spirosorbicillinol A, B and C, trichotetronine, bisvertinol and a new metabolite, the $(R)$ - stereoisomer of vertinolide. Koninginins and $\alpha$ pyrones were isolated from T. koningiopsis and T. atroviride, respectively.

To determine the adverse health effects caused by fungi indoors, isolates obtained directly from built environments must be studied which was achieved here. Some of the dominant toxins isolated, chaetoglobosin A, chaetomugilin D, phomenone, andrastin A, walleminone, tryptophol, koninginin A and trichotetronine, are being utilized as analytical standards to continue toxicological experiments aimed at determining how fungal metabolites alter human lung biology. These secondary metabolites encompass each the genera investigated, have known bioactivities and exhibit the diversity of fungal natural products. Information from these experiments will contribute to a more comprehensive understanding of the mechanism for nonatopic asthma associated with exposure to mold and dampness indoors. 


\section{ACKNOWLEDGMENTS}

The funding that made this project possible was provided by the Natural Sciences and Engineering Council of Canada (NSERC).

Most importantly, I want to express my gratitude to my supervisor Dr. J. David Miller for presenting me with the opportunity of working within his group and continued mentorship throughout my graduate studies. I appreciate that you allowed me to work through this fascinating project on my own but were always available to answer my questions or provide advice without hesitation. These experiences provided me with an understanding of how to do things the right way. I apologize for the so called "McMullin induced delays".

Secondly, I would like to thank Dr. Mark Sumarah who was tasked with teaching me about natural product chemistry but taught me more life lessons instead. I have not forgotten the important ones. Thank you for your continued advice, good times and all of the conversations, particularly the ones calming me down when I get worked up.

I need to thank Dr. Tienabe Nsiama for imparting his chemistry knowledge, convincing me to perform organic reactions on our precious compounds and always willing to offer his assistance; it was a pleasure working with you.

I sincerely thank and appreciate the efforts of Don Belisle, Paracel Inc., for providing indoor isolates, Dr. Dan Sørensen, McMaster University, for acquiring high quality NMR data that made many structural elucidations possible and for the invitation to Hamilton, Dr. Keith Seifert 
and Dr. Tharcisse Barasubiye, Agriculture and Agri-Food Canada, for providing fungal identifications and depositing many of the studied strains, Dr. Barbara Blackwell, Agriculture and Agri-Food Canada, for taking the time to help me decipher NMR spectra, Dr. Jeffery Manthorpe for his guidance with organic reactions and all the invitations to play hockey. A special thank you to Dr. Sue Twine, National Research Council, for not only facilitating mass spectrometry analysis but for writing me so many recommendation letters and convincing me to go to graduate school in the first place.

I not only thank but appreciate all of the past and present Miller group members that have helped me along the way and made this an enjoyable experience: Samantha Frasz, Blake Green, Natacha Provost, Natasha Prince, Gina Parker, Luke Johnson, Susan Richardson, Tamara Desroches, Shari Levac, Greg Slack and Aaron Wilson. I suppose I must apologize to a couple of you who know who you are for your retention within the group. The many friendships made while working in the Steacie building made the whole process a great personal experience. Particularly true of anyone who wanted to go for "a" drink.

I finally want to thank my family who has supported me throughout this endeavor. I think we can all agree, no one would have seen me here a decade ago, but I'm happy I am. 


\section{TABLE OF CONTENTS}

$\begin{array}{lr}\text { ABSTRACT } & 2\end{array}$

$\begin{array}{lr}\text { ACKNOWLEGMENTS } & 4\end{array}$

$\begin{array}{ll}\text { LIST OF FIGURES } & 7\end{array}$

$\begin{array}{ll}\text { LIST OF TABLES } & 11\end{array}$

$\begin{array}{ll}\text { SCENTIFIC CONTRIBUTIONS } & 13\end{array}$

$\begin{array}{ll}\text { CHAPTER I - GENERAL INTRODUCTION } & 15\end{array}$

CHAPTER II - ISOLATION, CHARACTERIZATION AND 40

QUANTIFICATION OF CHAETOGLOBOSINS AND AZAPHILONES

FROM CHAETOMIUM GLOBOSUM

CHAPTER III - ISOLATION AND STRUCTURAL ELUCIDATION OF

SECONDARY METABOLITES FROM PENICILLIUM CORYLOPHILUM

CHAPTER IV - CHARACTERIZATION OF METABOLITES FROM

WALLEMIA SEBI

CHAPTER V - ISOLATION AND STRUCTURAL ELUCIDATION OF SECONDARY METABOLITES FROM TRICHODERMA CITRINOVIRIDE, T. KONINGIOPSIS AND T. ATROVIRIDE

CHAPTER VI - GENERAL DISCUSSION

LITERATURE CITED 


\section{LIST OF FIGURES}

Figure Title

Page

Figure 1.1: Structure of the polyketides aflatoxin B1, zearalenone and griseofulvin

Figure 1.2: Structure of the sesquiterpenes deoxynivalenol and PR toxin

Figure 1.3 Structure of cinnaminc acid and roquefortine $\mathrm{C}$

Figure 1.4: Scheme of secondary metabolism

Figure 1.5: Structures of secalonic acid D, meleagrin and chrysogine isolated from either P. chrysogenum or P. rubens

Figure 1.6: Structures of the carcinogenic metabolites sterigmatocystin and 5methoxysterigmatocystin from $A$. versicolor and the trichothecene trichodermol from S. chartarum chemotype A and Tricoderma spp.

Figure 2.1: C. globosum grown on 2\% MEA

Figure 2.2: Structures of the toxin chetomin and purple pigment cochliodinol

Figure 2.3: Structures of chaetoglobosin A and C

Figure 2.4: Structures of chaetoviridin A-D

Figure 2.5: Structures of chaetomugilin A, D, I and M

Figure 2.6: Structures of chaetoglobosin U and chaetoglobin A

Figure 2.7: Structure of chaetoglobosin A

Figure 2.8: Structure of chaetoglobosin F

Figure 2.9: Structure of chaetoglobosin $\mathrm{C}$

Figure 2.10: Structure of chaetomugilin D

Figure 2.11: Structure of chaetoviridin A

Figure 2.12: HRESIMS spectra of chaetoglobosin A and chaetomugilin D

Figure 2.13: HPLC chromatogram of C. globosum DAOM 240349 filtrate extract at 
Figure 2.14: Calibration plots for chaetoglobosin A and chaetomugilin D 56

Figure 2.15: Structure of 4'-epi- $N$-2-hydroxyethyl-azachaetoviridin A 59

Figure 2.16: Observed COSY and key HMBC correlations for compound 4'-epi- $N$ - $\quad 60$ 2-hydroxyethyl-azachaetoviridin A

Figure 2.17: Structure of $N$-2-butyric-azochaetoviridin E $\quad 63$

Figure 2.18: Structure of isochromophilone XIII

Figure 3.1: P. corylophilum growing on 2\% MEA $\quad 80$

Figure 3.2: Structure of the iscohroman DHMI isolated and its synthetic derivative $\quad \mathbf{8 1}$ 8-methoxy-DHMI

Figure 3.3: Structure of the sesquiterpene phomenone and the isocoumarins $(+)-$ orthosporin and citreoisocoumarinol

Figure 3.4: Structures of citrinin, epoxyagroclavine-I and decarestrictine D 83

Figure 3.5: Structure of (1S,3S)-1,6,8-trihydroxy-3-(7-hydroxyheptyl) isochroman- $\quad \mathbf{8 5}$ 7-carboxylic acid

Figure 3.6: Methylation of compound 3.1 to its tetramethyl derivative 3.5 with diazomethane

Figure 3.7: Structure of CJ- 12,373 from an unidentified Penicillium species $\quad 87$

Figure 3.8: Structure of (1S,3S)-1,6,8-trihydroxy-3-((R)-6-hydroxyheptyl) $\mathbf{8 8}$ isochroman-7-carboxylic acid

Figure 3.9: $\Delta \delta\left(\delta_{\mathrm{S}}-\delta_{\mathrm{R}}\right)$ values (ppm) for the MTPA ester of compound 3.2 8

Figure 3.10: Structure of (S)-6,8-dihydroxy-3-(7-hydroxyheptyl) isochroman-7- 90 carboxylic acid

Figure 3.11: Structure of $(S)-6,8$-dihydroxy-3-((R)-6-hydroxyheptyl) isochroman-7carboxylic acid

Figure 3.12: Observed COSY and key HMBC correlations for 3.4 that are representative of the four new isochromans

Figure 3.13: Structure of (1S,3S)-methyl 3-(7-hydroxyheptyl)-1,6,8- 
Figure 3.14: Structure of 6-((2S,4R)-2,4-dihydroxyundecyl)-4-hydroxy-3-methyl2H-pyran-2-one

Figure 3.15: Structure of 6-((2S,4R)-2,4-dihydroxyundecyl)-4-hydroxy-3-methyl-

2H-pyran-2-one

Figure 3.16: Observed COSY and key HMBC correlations for 3.7 that are representative of the three new $\alpha$-pyrones

Figure 3.17: Structure of 4-hydroxy-3-methyl-6-((2S,4R)-2,4,11-trihydroxyundecyl)2H-pyran-2-one

Figure 3.18: Structure of phomenone

Figure 3.19: Structure of andrastin A

Figure 3.20: Structure of citreohybridonol

Figure 3.21: Structure of koninginin A

Figure 3.22: Structure of koninginin E

Figure 3.23: Structure of koninginin G

Figure 4.1: $W$. sebi growing MEA

Figure 4.2: Secondary metabolites previously reported from $W$. sebi

Figure 4.3: Structure of walleminone

Figure 4.4: Structure of wallimidione

Figure 4.5: Structure of tryptophol

Figure 4.6: Structure of tryptophol acetate

Figure 4.7: Structure of phenylacetic acid

Figure 4.8: Structure of $p$-hydroxybenzaldehyde

Figure 5.1: Structures of the trichothecenes trichodermin and harzianum A

Figure 5.2: Structures of gliotoxin and viridin

Figure 5.3: Structures of trichoviridine, isonitrin B, and a isocyanide isolated as a its methyl ester 
Figure 5.4: Structures of the simple pyrone, pyrone 6- pentyl-2H-pyran-2-one, koninginin A, sorbillin and bisvertinol

Figure 5.5: Structure of $(R)$ - vertinolide

Figure 5.6: Structure of spirosorbicillinol A

Figure 5.7: Structure of spirosorbicillinol B

Figure 5.8: Structure of spirosorbicillinol C

Figure 5.9: Structure of trichotetronine

Figure 5.10: Structure of bisvertinol

Figure 5.11: Structure of bisvertinol, bisvertinolone and dihydrobisvertinol

Figure 5.12: Structure of sorbicillin and sorbicillinol

165

Figure 5.13: Structure of various bisorbicillinoids derived from their postulated precursor sorbicillin illustrating their structural diversity

Figure 5.14: Structures of citrantifidiene and citrantifidiol

Figure 5.15: Structure of koninginin B

Figure 5.16: Structure of koninginin D

Figure 5.17: Structure of koninginin F

Figure 5.18: Structure of pyrone 6- pentyl-2H-pyran-2-one

Figure 5.19: Structure of 6- pent-1-enyl-2H-pyrane-2-one 


\section{LIST OF TABLES}

Table Title

Page

Table 2.1: ${ }^{1} \mathrm{H}(400 \mathrm{MHz})$ and ${ }^{13} \mathrm{C}$ NMR $(100 \mathrm{MHz})$ data for chaetoglobosin A, F and C

Table 2.2: ${ }^{1} \mathrm{H}(400 \mathrm{MHz})$ and ${ }^{13} \mathrm{C}$ NMR $(100 \mathrm{MHz})$ data for chaetomugilin $\mathrm{D}$ in $\mathrm{CD}_{3} \mathrm{CN}$

Table 2.3: ${ }^{1} \mathrm{H}(400 \mathrm{MHz})$ and ${ }^{13} \mathrm{C}$ NMR $(100 \mathrm{MHz})$ data for chaetoviridin A in $\mathrm{CD}_{3} \mathrm{CN}$

Table 2.4: Metabolite production by Canadian strains of C. globosum in the filtrate and mycelium extracts

Table 2.5: ${ }^{1} \mathrm{H}(400 \mathrm{MHz})$ and ${ }^{13} \mathrm{C}$ NMR $(100 \mathrm{MHz})$ data for 2.6 in $\mathrm{CD}_{3} \mathrm{CN}$

Table 2.6: ${ }^{1} \mathrm{H}(400 \mathrm{MHz})$ and ${ }^{13} \mathrm{C}$ NMR $(100 \mathrm{MHz})$ data for 2.7 in $\mathrm{CD}_{3} \mathrm{OD}$

Table 2.7: ${ }^{1} \mathrm{H}(400 \mathrm{MHz})$ and ${ }^{13} \mathrm{C}$ NMR $(100 \mathrm{MHz})$ data for 2.8 in $\mathrm{CD}_{3} \mathrm{CN}$

Table 2.8: Inhibition of B. subtilis, P. putida and S. cerevisiae by chaetoglobosins and azaphilones

Table 2.9: C. globosum strains examined for metabolite production

Table 3.1: ${ }^{1} \mathrm{H}(700 \mathrm{MHz})$ and ${ }^{13} \mathrm{C}$ NMR Data $(175 \mathrm{MHz})$ for new isochromans 3.13.4 in $\mathrm{CD}_{3} \mathrm{OD}$

Table 3.2: ${ }^{1} \mathrm{H}(700 \mathrm{MHz})$ and ${ }^{13} \mathrm{C}$ NMR Data $(175 \mathrm{MHz})$ for new $\alpha$-pyrones 3.6-3.8 in $\mathrm{CD}_{3} \mathrm{OD}$

Table 3.3: ${ }^{1} \mathrm{H}(400 \mathrm{MHz})$ and ${ }^{13} \mathrm{C}$ NMR Data (100 MHz) phomenone in $\mathrm{CD}_{3} \mathrm{OD}$

Table 3.4: ${ }^{1} \mathrm{H}(400 \mathrm{MHz})$ and ${ }^{13} \mathrm{C}$ NMR Data $(100 \mathrm{MHz})$ for andrastin $\mathrm{A}$ and

Table 3.5: ${ }^{1} \mathrm{H}(400 \mathrm{MHz})$ and ${ }^{13} \mathrm{C}$ NMR Data $(100 \mathrm{MHz})$ for koninginin A, koninginin $\mathrm{E}$ and koninginin $\mathrm{G}$ in $\mathrm{CD}_{3} \mathrm{OD}$

Table 3.6: Metabolite production by indoor $P$. corylophilum strains studied

Table 3.7: Indoor P. corylophilum strains examined for metabolite production

Table 4.1: ${ }^{1} \mathrm{H}(400 \mathrm{MHz})$ and ${ }^{13} \mathrm{C}$ NMR Data $(100 \mathrm{MHz})$ walleminone in $\mathrm{CD}_{3} \mathrm{OD}$

Table 4.2: ${ }^{1} \mathrm{H}(400 \mathrm{MHz})$ and ${ }^{13} \mathrm{C}$ NMR Data $(100 \mathrm{MHz})$ wallimidione in $\mathrm{CD}_{3} \mathrm{OD}$ 
Table 4.3: Metabolite production by indoor $W$. sebi strains examined

Table 4.4: $W$. sebi strains examined for metabolite production

Table 5.1: ${ }^{1} \mathrm{H}(400 \mathrm{MHz})$ and ${ }^{13} \mathrm{C}$ NMR Data $(100 \mathrm{MHz})$ for $(R)$ - vertinolide in $\mathrm{CD}_{3} \mathrm{OD}$

Table 5.2: ${ }^{1} \mathrm{H}(400 \mathrm{MHz})$ and ${ }^{13} \mathrm{C}$ NMR Data (100 MHz) for spirosorbicillinol A, B and $\mathrm{C}$ in $\mathrm{CD}_{3} \mathrm{OD}$

Table 5.3: ${ }^{1} \mathrm{H}(400 \mathrm{MHz})$ and ${ }^{13} \mathrm{C}$ NMR Data $(100 \mathrm{MHz})$ for trichotetronine in $\mathrm{CD}_{3} \mathrm{OD}$

Table 5.4: ${ }^{1} \mathrm{H}(400 \mathrm{MHz})$ and ${ }^{13} \mathrm{C}$ NMR Data (100 MHz) for bisvertinol in $\mathrm{CD}_{3} \mathrm{CN}$

Table 5.5: ${ }^{1} \mathrm{H}(400 \mathrm{MHz})$ and ${ }^{13} \mathrm{C}$ NMR Data (100 MHz) for koninginins A, B, D and $\mathrm{F}$ in $\mathrm{CD}_{3} \mathrm{OD}$

Table 5.6: ${ }^{1} \mathrm{H}(400 \mathrm{MHz})$ and ${ }^{13} \mathrm{C}$ NMR Data (100 MHz) pyrone 6- pentyl-2Hpyran-2-one in $\mathrm{CD}_{3} \mathrm{OD}$

Table 5.7: Indoor Trichoderma strains examined for metabolite production 


\section{SCIENTIFIC CONTRIBUTIONS}

\section{Peer Reviewed Contributions}

Tamara C. Desroches, David R. McMullin and J. David Miller (2014) Extrolites of Wallemia sebi, a very common fungus in the built environment. Indoor Air. Accepted.

David R. McMullin, Tienabe K. Nsiama and J. David Miller (2014) Secondary metabolites from Penicillium corylophilum isolated from damp buildings. Mycologia. Accepted.

David R. McMullin, Tienabe K.Nsiama and J. David Miller (2014) Isochromans and $\alpha$-pyrones isolated from Penicillium corylophilum. Journal of Natural Products, 77: 206-212.

David R. McMullin, Mark W. Sumarah, Barbara A. Blackwell, J. David Miller (2013) New azaphilones from Chaetomium globosum isolated from the built environment. Tetrahedron Letters, 54: 568-572.

David R. McMullin, Mark W. Sumarah, J. David Miller (2013) Chaetoglobosins and azaphilones produced by Canadian strains of Chaetomium globosum isolated from the indoor environment. Mycotoxin Research, 1: 47-54.

Susan M. Twine, Christopher W. Reid, Annie Aubry, David R. McMullin, Kelly M. Fulton, John Austin, Susan M. Logan (2009) Motility and flagellar glycosylation in Clostridium difficile Journal of Bacteriology, 19: 7052-7062.

Susan M. Twine, Catherine J. Paul, Evgeny Vinogradov, David J. McNally, Jean-Robert Brisson, James A. Mullen, David R. McMullin, Harold C. Jarrell, John W. Austin, John F. Kelly, Susan M. Logan (2008) Flagellar glycosylation in Clostridium botulinum. FEBS Journal, 17: 44284444.

\section{Non Peer Reviewed Contributions}

J. David Miller, Susan N. Richardson, David R. McMullin, Justin Falardeau (2013) Literature review on deoxynivalenol, zearalenone, T-2/H-T2 toxins, fumonisins and the fungi that produce them in Canada with a commentary on Alternaria alternate toxins in grains and the potential for Aspergillus flavus to become a problem in Ontario corn. Industry Branch, AAFC, Ottawa. pp. 233.

\section{Poster Presentations}

David R. McMullin, Tienabe K. Nsiama, J. David Miller (2013) New secondary metabolites produced by indoor strains of Penicillium corylophilum.

Gordon Research Conference: Mycotoxins and Phycotoxins. Easton, Massachusetts, USA. June 2013. 
David R. McMullin, Natasha C. Prince, Farah Hosseinian, J. David Miller (2012) Preliminary evidence that phenolic distribution is associated with tolerance to fusarium head blight.

MycoRed North America. Ottawa, Ontario, Canada. June 2012.

David R. McMullin and David Miller (2011) Isolation and quantification of secondary metabolites produced by Chaetomium globosum obtained from the built environment.

Gordon Research Conference: Mycotoxins and Phycotoxins. Waterville, Maine, USA. June 2011.

David R. McMullin and David Miller (2009) Characterization of secondary metabolites from Chaetomium globosum and Wallemia sebi.

Gordon Research Conference: Mycotoxins and Phycotoxins. New London, New Hampshire, USA. June 2009. 


\section{CHAPTER I}

\section{GENERAL INTRODUCTION}




\section{Fungi}

Fungi rank second only to insects in estimated species biodiversity. Conservative estimates indicate that there are likely more than 1.5 million fungal species where only a small percentage $(\sim 5 \%)$ has been described (Hawksworth 2001). This is more than five and fifty times the estimated number of plant and bacterial species respectively on the planet (Hawksworth 1991). However, using high throughput screening methods, a more recent estimate suggests up to 5.1 million fungal species may exist (Blackwell 2011). Coming from one of the most successful eukaryotic lineages, fungi are capable of thriving in competitive environments. Fungi have been shown to inhabit almost all niches on Earth including soil, marine systems, in cold and salty environments and as endophytes. Some of these very adaptable organisms have the ability to slow their metabolism when conditions do not permit growth and may stay dormant until favorable conditions return (Watling 2003).

From the time of Linnaeus, fungi have been categorized according to the nature of their reproduction as Ascomycetes, Basidiomycetes or Phycomycetes. Fungi may reproduce either asexually or sexually however, environmental conditions may alter the prevalence of a particular sexual state. Among other problems, this led to the situation where the same fungus could have several names because many fungi have both sexual (teleomorphic) and asexual (anamorphic) stages (Kendrick 2001). This has required a more extensive use of molecular methods. Today, molecular systematics plays a major role in delimiting species concepts. The ultimate expression of this trend was the so-called Amsterdam declaration which has allowed for a one fungus one name system (Hawksworth et al. 2011). 
Fungal spores are specialized structures for reproduction and dispersal. Asexual spores are produced by mitotic division whereas sexual spores are derived from meiosis causing genetic recombination (Kendrick 2001). Liberated spores may be dispersed by wind, water, agitation, insects, animals or humans allowing filamentous fungi to propagate into new environments including anthropogenic structures (Flannigan et al. 2001). These spores may remain dormant for an extended period of time until specific environmental conditions such as temperature, nutrient availability or water activity are satisfied (Smith and Berry 1974). If suitable environmental criteria are achieved, the spore will germinate into a germ tube that grows into a single filament, a hypha. The hypha branches to create hyphae where additional branching and aggregation of hyphae generates a mycelium (Watling 2003). Fungal spores constitute a large portion of both indoor and outdoor air and have been directly detected from the two separate environments. The spores found in buildings without mold damage are primarily derived from outdoor sources (Adams et al. 2013; Foto et al. 2005; Miller et al. 2008).

As heterotrophs, fungi obtain their nutrients by degrading organic matter through the excretion of degradative enzymes making them the planets natural recyclers. The uptake of carbohydrates and other accessible nutrients including nitrogen, phosphorus, potassium, sulphur, iron, magnesium and calcium is required for their growth. They lack chlorophyll and are not capable of photosynthesis. Fungi generally have a semi-rigid cell wall containing chitin, cellulose-like substances and glucans, that are permeable to water and solutes in solution (Kendrick 2000) 
Besides recycling decaying organic matter, an ecologically vital role, fungi have been exploited as a direct source of food, fermentation of alcoholic beverages, production of industrial enzymes, agricultural bio-control agents and pharmaceutically active compounds for the benefit of the human species. Two notable examples include the antibiotic penicillin and cholesterol lowering compounds, statins (Gloer 2007). However, fungi cause various unwanted, deleterious effects that directly affect human populations. Fungi are capable of causing mycotic infections, spoiling food and both crop and animal diseases that are acerbated by the production of mycotoxins. The production of mycotoxins is of particular importance due to their widespread occurrence as contaminants of food for both humans and livestock as well as their potent bioactivities (Jarvis and Miller 2005). Fungi found indoors may affect population health by generating spores, liberating hyphal fragments and aerosols comprised of several biologically active compounds including allergens, low molecular weight compounds, and $\beta$-1, 3-D-glucan (Nielsen et al. 2003; Miller et al. 2010; Rand et al. 2010).

\section{Primary and Secondary Metabolism}

All organisms synthesize and degrade organic and inorganic materials by series of anabolic and catabolic enzyme mediated reactions collectively known as metabolism (Betina 1995). Primary metabolism occurs in all living organisms as it is essential for growth and reproduction. Major primary metabolic pathways include glycolysis, Krebs cycle, beta oxidation, amino acid and nucleic acid production. The intermediates and end products of these biochemical pathways are known as primary metabolites and are required for the anabolism and catabolism of essential biomacromolecules including proteins, fatty acids, carbohydrates and nucleic acids (Mann 1987). 
Among the more fascinating properties of fungi is their ability to synthesize a wide variety of secondary metabolites typically defined as small molecules that are not necessary for normal growth or development (Fox and Howlett 2008). The functions that are observed or proposed today may not be the same as the evolutionary forces that resulted in their appearance (Davies 1990). Demain and Fang (2000) noted that these metabolites function (i) as competitive weapons used against other bacteria, fungi, amoebae, plants, insects, and large animals; (ii) as metal transporting agents; (iii) as agents of symbiosis between microbes and plants, nematodes, insects, and higher animals or other possible ecological functions. There is now strong evidence supporting secondary metabolites that allow organisms, particularly microorganisms, to carve out an ecological niche (Gloer 2007; Keller et al. 2005). Their ecological significance is demonstrated by the production of, often potent, secondary metabolites by fungi, bacteria, plants and other organisms as a stress response to other fungi, insects or animals. Fungi will inhabit an ecological niche provided there are nutrients available, however, these resources will be competed for by other organisms. Some form of interference competition by either behavioral or chemical interaction is hypothesized to occur before these resources can be exploited (Wicklow 1981). The production of toxic secondary metabolites by an organism would directly affect a competitor's ability to access the resource, increasing their own chance for survival. Fungi may colonize a resource and produce secondary metabolites that render the nutrient substrate unappealing to animals or other microorganisms.

This can be exemplified by fungal endophytes which live in plant vascular or leaf tissue without causing damage. Secondary metabolites produced by grass endophytes reduce populations of 
herbivorous insects that attack the plants thus increasing plant fitness. The endophytic, bioactive compounds allow the plant to defend itself directly affecting its fitness (Clay 1988; Cragg and Newman 2005). Similar observations have been made with regard to conifer endophytes. Foliar endophytes have been shown to produce an array of compounds toxic to herbivorous pests such as the Eastern spruce budworm. These compounds accumulate in the needles and reduce the growth rate of insect conferring increased tolerance to pests (Miller 2011; Sumarah et al. 2009).

As one or more environmental nutrient becomes limited, primary metabolites accumulate that can be incorporated into secondary metabolites. This includes acetyl-CoA, shikimic acid, mevalonic acid and amino acids (Mann 1987). As alluded to above, the production of secondary metabolites may also have to be induced by an environmental stressor such as a competitor or nutrient limitation (e.g. carbon or nitrogen). However, secondary metabolism is differentiated from primary metabolism since the organism must possess the required biosynthetic genes that can be strain specific (Miller et al. 1991).

Despite the vast structural diversity of secondary metabolites, the majority are derived from the mentioned simple primary metabolites. Acetyl-CoA is derived from the oxidative decarboxylation of pyruvic acid from the glycolytic pathway or by the beta hydroxylation of fatty acids. It is subsequently used in the acetate pathway to produce a variety of chemicals including polyketides, fatty acids, phenols and isoprenoids. Shikimic acid is derived from erythrose-4-phosphate (pentose phosphate pathway) and phosphoenolpyruvate (glycolytic pathway). Aromatic compounds including phenols, lignans, cinnaminic acid, alkaloids and amino acids (tyrosine, tryptophan or phenylalanine) are produced from the shikimate pathway. 
Mevalonic acid is generated from the condensation of three acetyl-CoA molecules. The mevalonate pathway yields structurally diverse terpenes and steroid precursors.

Polyketides are formed through the sequential condensation of acetate groups catalyzed by polyketide synthases (PKS's). Fungal polyketides are synthesized by type I PKS's that are multidomain enzyme complexes that function in a homologous manner compared to eukaryotic fatty acid synthases. Both pathways proceed by the addition of $\mathrm{C}_{2}$ units. Polyketides initially contain alternating $\mathrm{CO}-\mathrm{CH}_{2}$ groups as opposed to a saturated $\mathrm{CH}_{2}$ chain present in fatty acids (Staunton and Weissman 2001). Even from this simple precursor (acetate), great structural variability can be achieved. Polyketide synthesis begins with acetyl-CoA and the subsequent $\mathrm{C}_{2}$ units are derived from malonyl-CoA, an activated form of acetyl-CoA. This is produced by the carboxylation of acetyl-CoA. These large enzyme complexes then carry out a series of repeated biosynthetic reactions. An acyl carrier protein (ACP) is responsible for the transfer of various intermediates to different active sites found in the PKS complex. More specifically, a phosphopantethiene group tethered to a specific ACP serine residue facilitates the movement of intermediates in the complex. Once the first acetyl group is bound to a specific acetyl transferase serine residue, it is transferred to the free thiol group of the ACP and is subsequently transferred the ketoacyl synthase (KS). Now free, the thiol group of the ACP performs a nucleophilic attack on the carbonyl carbon of the malonyl attached to the malonyl transferase creating malonyl-ACP. Decarboxylation of the unit attached to the ACP creates a very nucleophilic carbanion that attacks the carbonyl carbon of the acetyl group connected to the KS. Subsequent additions of malonyl-CoA subunits utilizing the same enzymatic machinery elongates the chain in a head to tail fashion two carbons at a time until the polyketide chain is hydrolyzed from the ACP. The 
linear polyketide chain is additionally oxidized, reduced, rearranged and modified (e.g. methylated) by a variety of enzymes to generate the vast structurally diversity of polyketides.

Oxidative modifications by cytochromes and dehydrogenases are the most common enzymes that increase the structural diversity of these secondary metabolites. The mycotoxins aflatoxin B1 and zearalenone as well as the antifungal compound griseofulvin are examples of important polyketides; figure 1.1.
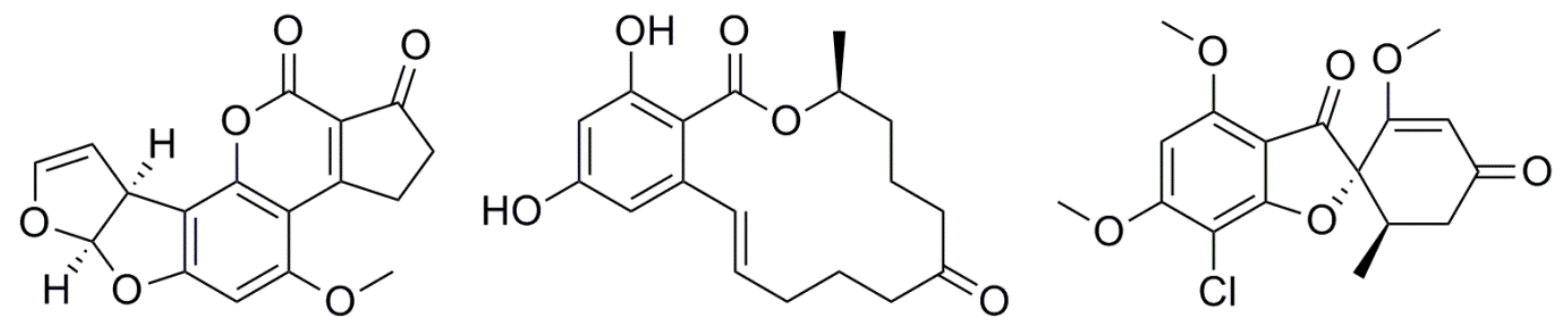

Figure 1.1: Structure of the polyketides aflatoxin B1, zearalenone and griseofulvin.

Terpenes are a very large class of secondary metabolites produced by fungi as well as plants and other animals. They can be produced by some insects and serve as precursors for steroids in many organisms using mevalonic acid as the key intermediate. All of this structural diversity originates from the simple hydrocarbon isoprene, $\mathrm{C}_{5} \mathrm{H}_{8}$. Terpenes can be classified by the number of $\mathrm{C}_{5}$ isoprene units that are incorporated into them as hemiterpenese (1 unit), monoterpenes ( 2 units), sesquiterpenes ( 3 units), diterpenes (4 units), sesterterpenes (5 units) and so on. However, isoprene cannot be directly incorporated into terpenes, the activated forms isopentenyl pyrophosphate (IPP) and dimethylallyl pyrophosphate (DMAPP) derived from mevalonic acid are. These intermediates are derived from two independent pathways, the 
mevalonic acid or HMG-CoA reductase pathway, which takes place in the cytosol, and the MEP pathway, that occurs in the plastids of plants and protozoa (Hanson 2007).

In the cytosolic pathway used by fungi, a thiolase converts two acetyl-CoA to acetoacetyl-CoA. An additional acetyl-CoA is condensed with acetoacetyl-CoA to form 3-hydroxy-3methylglutaryl-CoA (HMG-CoA) which is subsequently reduced by HMG reductase to mevalonic acid. Mevalonic acid is then phosphorylated by two kinases to 5pyrophosphomevalonate. Decarboxylation of this in the presence of ATP by pyrophosphomevalonate decarboxylase yields IPP. The MEP pathway found in plant plastids utilizes different precursors, pyruvate and glyceraldehyde-3-phosphate to yield IPP and DMAPP. These isoprenoid precursors can be condensed together in a step wise head to tail fashion with various numbers of $\mathrm{C}_{5}$ units incorporated to produce structurally diverse metabolites. These can additionally be modified by terpenoid synthases that cyclize, add prenyl moieties, oxidize or reduce the terpenoid precursors to increase their structural diversity. The trichothecene mycotoxin deoxynivalenol and eremophilane sesquiterpene PR toxin are illustrated in figure 1.2, both of which are sesquiterpenes.
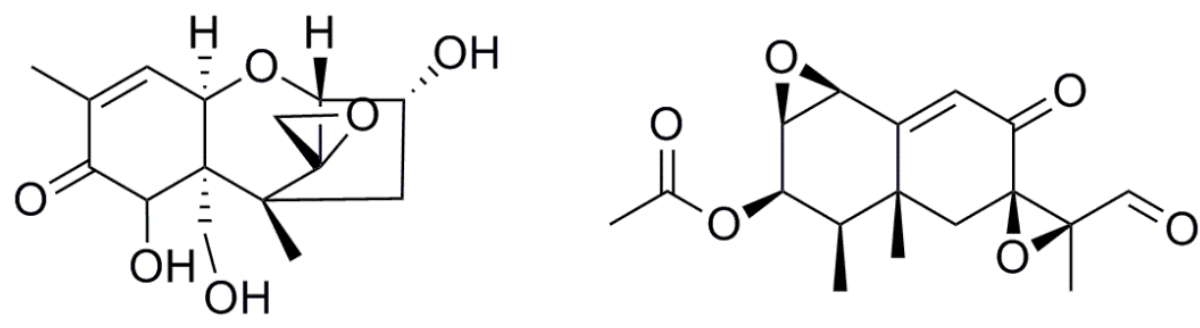

Figure 1.2: Structure of the sesquiterpenes deoxynivalenol and PR toxin.

The shikimate pathway produces a variety of aromatic compounds including some very important primary metabolites such as the amino acids phenylalanine, tyrosine and tryptophan. 
The biosynthesis of shikimic acid starts with the condensation of erythrose-4-phosphate derived from the pentosephosphate cycle and phosphoenolpyruvic acid from glycolysis. These two metabolites are essential for the metabolism carbohydrates. Shikimate derived compounds include coumarins, cinnaminc acid and other phenolic acids that typically do not contain nitrogen. Shikimate intermediates may also be directly incorporated into secondary metabolites. Figure 1.3 shows the structure of cinnamic acid as well as roquefortine $\mathrm{C}$ that is derived directly from tryptophan, histidine and dimethylallylpyrophosphate.
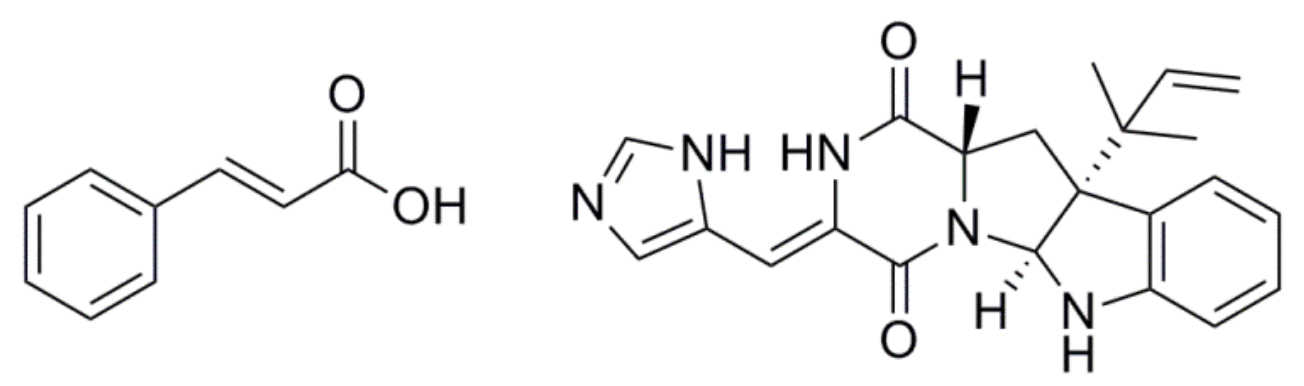

Figure 1.3: Structure of cinnamic acid and roquefortine $C$.

The principal metabolites derived from amino acids include alkaloids, peptides or metabolites with mixed biosynthesis. Compounds such as cocaine, ephedrine and nicotine are all examples of pharmacologically active alkaloids. Alkaloids contain at least one nitrogen atom and are derived primarily from the basic amino acids ornithine and lysine or the aromatic amino acids phenylalanine, tyrosine, and tryptophan which are formerly shikimate derived compounds (Mann 1987). Fungal secondary metabolites also include peptides that are synthesized from non- 


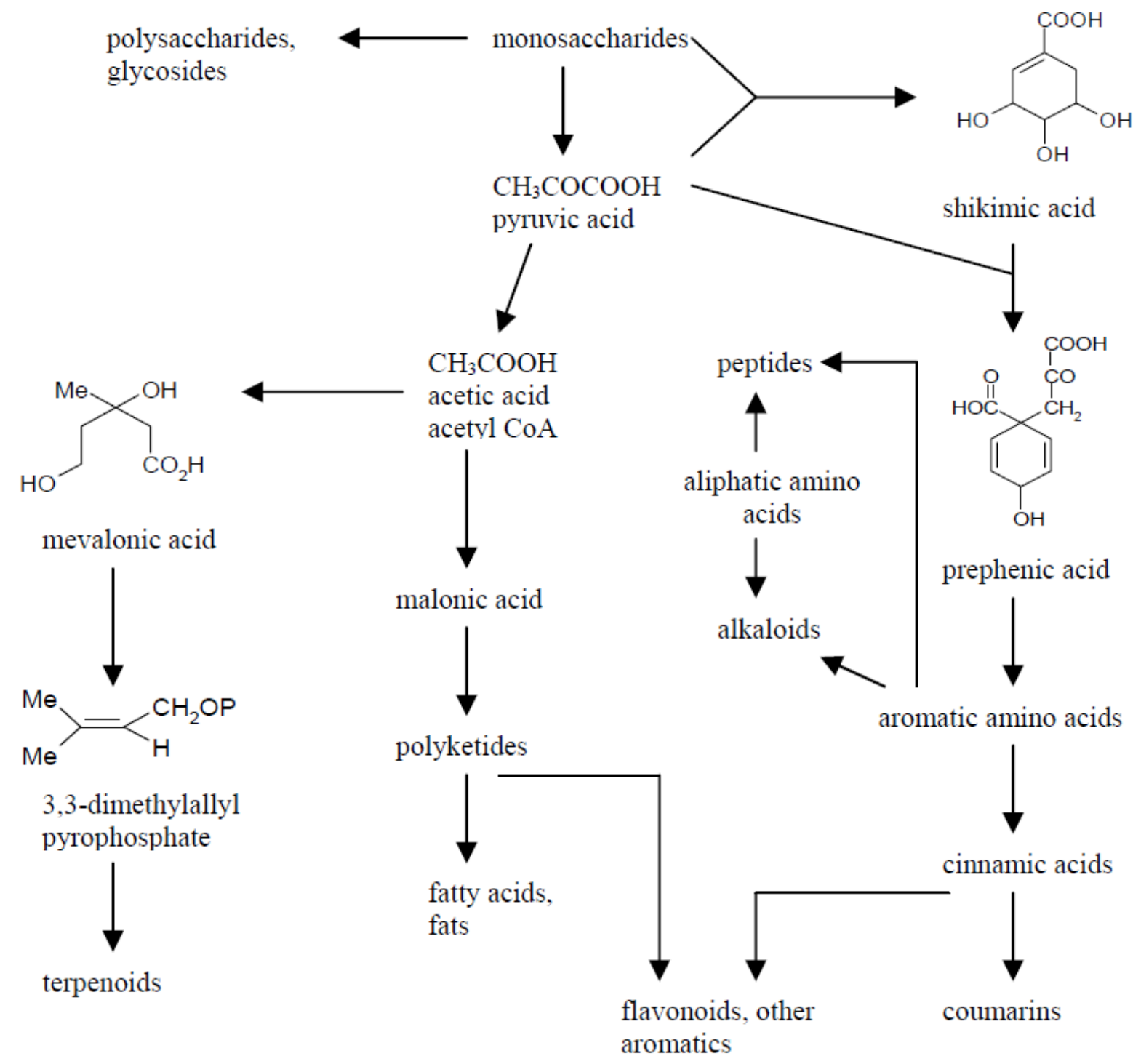

Figure 1.4: Scheme of secondary metabolism (adapted from Torssell 1983).

ribosomal pathways. They can either be linear or cyclic. Examples of cyclic peptides are the enniatins produced by Fusarium species (Sy-Cordaro et al. 2012) and the immunosuppressant drug cyclosporine (Bushley et al. 2013). The genus Trichoderma has so far been the richest source of modified non-ribosomal peptides, known as peptaibols, that contain both proteogenic and non-proteogenic amino acids (Leitgeb et al. 2007). The antibiotic penicillin is an example of 
a modified tripeptide. The nephrotoxic mycotoxin ochratoxin A and the cytochalasin chaetoglobosins are examples of secondary metabolites arising from a mixed polyketide-amino acid biosynthesis.

\section{Chemotaxonomy}

As described above, filamentous fungi are capable of synthesizing a vast number of structurally diverse secondary metabolites. Chemotaxonomy is a means of classifying or identifying organisms based on their chemical diversity and is often limited to qualitative or quantitative profiles of fatty acids, proteins, carbohydrates or secondary metabolites. For fungi, a secondary metabolite profile to a chemist encompasses all of the metabolites within an extract. These metabolites are typically produced by a limited number of fungal species within a genus, order or phylum meaning they cannot be applied to phylogenetic studies. However, the set of metabolites of a particular species are typically consistently produced (Larsen et al. 2005). Chemotaxonomy based on unique secondary metabolites within the profile and not a single chemical offers a means to differentiate fungal species (Frisvad et al. 2008). A chemotaxonomic approach utilizing secondary metabolites have been successfully applied to economically important Penicillium, Aspergillus and Fusarium species as well as lichens (Laresen et al. 2005). Secondary metabolite profiles are particularly useful differentiating species that are morphologically and physiologically similar as demonstrated with the P. roqueforti complex (Nielson et al. 2006).

When classifying fungi, a polyphasic approach incorporating secondary metabolite data, morphological, physiological and genetic characteristics should be utilized. A polyphasic 
approach will limit the number of systematic errors associated with fungal identifications. The literature is not short of erroneous claims of secondary metabolites produced by fungi. This can be caused by fungal misidentifications highlighting the importance of strains being deposited in recognized culture collections. This also ensures the correctly identified isolates are maintained properly. While fungal secondary metabolite production is typically species or even strain specific, the spontaneous production of atypical metabolites is not unheard of (Larsen et al. 2005). This is postulated to be due to poor growth conditions or improperly maintained strains. For these reasons, chemotaxonomic studies should utilize well characterized, deposited isolates (Frisvad et al. 2008). Polyphasic examinations of fungi have resulted in the taxonomic revisions of many fungal genera.

Advancements in analytical instrumentation including mass spectrometry, nuclear magnetic resonance and high performance liquid chromatography are allowing secondary metabolites to be mined like never before. Further, increased computing power with the ability to store and statistically evaluate large sets of chemical data will only increase the ability to probe fungal secondary metabolites. To properly investigate fungal chemotypes, secondary metabolite analytical standards derived from deposited isolates are required. Increased exploitation of fungi and their metabolites has many economic implications as they constitute a large number of pharmacologically active compounds and in the food sector due to the production of mycotoxins. Academically, a more complete description of the chemical profiles of fungi is of relevance as it is through the production of secondary metabolites that fungi interact with their environment. 


\section{Fungi in the Built Environment}

Modern buildings have been designed to be more energy efficient and are therefore constructed with excess insulation in a manner that limits ventilation rates compared to buildings constructed prior to the 1970's. While this "tightening" of North American dwellings and buildings was subsidized by governments, little consideration was given to the effects it would have on indoor air quality (Miller et al. 2007). These buildings are constructed with materials that are designed to absorb water however, when the amount of water vapor generated in a building exceeds its capacity to remove it, it is absorbed by the building materials. This promotes mold growth indoors (Johnson and Miller 2012; Miller et al. 2008) or may cause structural damage from wood rot. This is of particular importance from a human health perspective because individuals in industrial countries, including Canada, spend the majority of their time indoors (Leech et al. 2002).

Population studies over the last couple decades have demonstrated individuals inhabiting or working in damp and moldy buildings are at increased risk of adverse health effects caused by fungi. Dampness and mold in the built environment is associated with increased upper respiratory disease, allergic responses, asthma and many other non-specific symptoms such as airway infections, impaired immune function, and fatigue in both atopic and non-atopic individuals (Health Canada 2004; 2007; NIOSH 2012; WHO 2009). Allergy alone cannot explain this pattern of disease suggesting toxins produced by fungi play a role in the disease process (Mendell et al. 2011; Miller et al. 2010; Neveu et al. 2011; Rand et al. 2011). 
Fungal proteins may induce Type 1 allergic responses and non-immunoglobulin E ( $\operatorname{IgE})$ mediated histamine release. The form of glucan in the fungi most often referred to as molds, $\beta$ $(1,3)$-D-glucan, is able to trigger inflammatory responses that are similar to endotoxin exposure by activating the dectin receptor (Rand et al. 2010). Exposure to this particular triple helical glucan induces immunomodulatory changes in neutrophils, macrophages, eosinophils and increases levels of inflammatory biomarkers in both human and animal blood.

Fungi commonly found indoors are capable of synthesizing an array of structurally diverse secondary metabolites, most of which are non-volatile, indicating exposure is largely due to the inhalation of spores and mycelial fragments (Green et al. 2006; Salares et al. 2009). From agricultural experiences, inhalation exposures of mycotoxins can lead to human disease (Miller et al. 1991). Stachybotrys chartarum growing on straw has previously been demonstrated to cause disease in farm animals and workers by inhalation exposures to trichothecenes (Miller et al. 2003).

A large epidemiological study by Dales et al. (1991) indicated that the symptoms associated with indoor exposure to fungi could not be attributed to allergy alone and that secondary metabolites produced by fungi play an important role for "sick building syndrome". Additionally, asthma is normally associated with a genetic predisposition, known as atopy. However, individuals living or working for prolonged periods in damp and moldy buildings are at increased risk of developing asthma, i.e. non-atopic asthma (NIOSH 2012). This suggests that indoor inhalational exposure to fungal products is a mechanism for non-atopic asthma. This has been postulated to be due to the inflammatory effects caused by fungal metabolites present on spores or mycelial 
fragments. Inhalation exposures to secondary metabolites produced by fungi common on damp building materials that could be experienced in buildings with mold problems have been demonstrated to affect lung biology in vivo and in vitro (Akpina et al. 2013; Miller et al. 2010, Rand et al. 2010; Rand et al. 2011; Rand et al. 2013). Fungal spores and low doses of pure secondary metabolites from indoor fungi elicit acute lung inflammation in rodent models (Miller et al. 2010). Little is currently known about how fungal metabolites affect biologically relevant endpoints in lung cells however, induction of pro-inflammatory genes along with histopathological disruptions are observed in mouse alveolar cells at fungal toxin concentrations that can be experienced indoors, $4 \times 10^{-5} \mathrm{~mol} \mathrm{~kg}^{-1}$ lung (Miller et al. 2010). The physiological inflammation, molecular induction of inflammation and immunomodulatory effects caused by fungal metabolites on lung cells is still poorly understood biochemically.

\section{Fungal Contamination of Indoor Environments}

As previously mentioned, the species diversity of fungi is very high. However, the fungi that are associated with damp buildings or indoor air comprise a narrow group of species (Jarvis and Miller 2005). Common indoor fungi must be able to survive on the amount of biologically available water and nutrients made accessible from building materials. The lack of species variation indoors globally is most likely a reflection of the widespread use of similar building materials such as gypsum board in the developed world. Fungal spores originating from outdoors, primarily from soil, may enter the indoor environment by wind or various other vectors including pets, dirt, plants debris, or clothing. The germination of these spores indoors will be 
dependent upon various biotic and abiotic factors such as temperature, humidity, building material, nutrient availability, fungal age and species (Kildeso et al. 2003).

Fungi are capable of growing on many commonly used moist building materials. Damp building materials that support growth may result in the formation of a large fungal biomass, however if the material dries, this can promote spore and mycelial fragment dispersion (Nielsen 2003). Mold growth on water damaged plaster, concrete, wood, wallpaper, paint and gypsum is common whereas some species are found on more unlikely substrates such as fiberglass, brick, and linoleum. Painted plasterboard is an easily degraded carbon source but will not easily support mold growth unless damp (Andersen et al. 2011).

The water activity $\left(a_{\mathrm{w}}\right)$ of the damaged material is the most important factor determining if mold growth will be initiated and what species will proliferate. The $a_{\mathrm{w}}$ refers to the amount of biologically available water available in a substrate. It is defined as the vapor pressure of water on a particular substrate over the vapor pressure of pure water at the same temperature (Flannigan et al. 2001). The $a_{\mathrm{w}}$ value may be between 0-1.0 where the $a_{\mathrm{w}}$ of pure water is 1.0. The $a_{\mathrm{w}}$ of a particular building material, as well as temperature, are the most important abiotic factors affecting mold growth indoors as different fungi will tolerate different water activities (Andersen et al. 2011). Additionally, some molds will tolerate a lower $a_{\mathrm{w}}$ if the temperature is higher (Flannigan et al. 1991). Localized differences in ventilation and surface temperature are capable of generating microclimates of high $a_{\mathrm{w}}$ where the relative humidity is low. This makes relative humidity a poor indicator of mold growth indoors (Andersen et al. 2011). 
Moisture problems in the indoor environment can be caused by natural means such as flooding as a result of heavy rain, melting snow, sewer system overflow or human error (Andersen et al. 2011). Daily routine human activities such as showering, laundry and cooking are capable of causing water damage when vapor condenses on cold surfaces such as walls, windows and furniture. However, often the most serious mold problems indoors arise when moisture is allowed to accumulate due to insufficient ventilation or air circulation. Additional moisture problems arise from poorly constructed or old roofing, wall and floor construction or plumbing leaks. For many fungi, condensation on building materials is sufficient for colonization. The longer a building material has a $a_{\mathrm{w}}$ greater than 0.75 , the higher the risk of mold growth (AIHA 2008).

Fungi found in the built environment can be classified as primary, secondary or tertiary colonizers according to their ability to grow on substrates with different water activities (Andersen et al. 2011). Fungi that require a minimum $a_{\mathrm{w}}>0.90$ are referred to as hydrophilic or tertiary colonizers, $a_{\mathrm{w}} 0.80-0.89$ are mildly hydrophilic molds or secondary colonizers, and $a_{\mathrm{w}}<$ 0.80 are xerophilic fungi or primary colonizers (Miller et al. 2008).

Primary colonizers are capable of growing when the $a_{\mathrm{w}}$ of the damp buildings materials is $<0.8$. This includes many Penicillium, Eurotium and Aspergillus species. Primary colonizers commonly identified indoors include Penicillium chrysogenum, $P$. corylophilum, $P$. commune, $P$. brevicompactum, Aspergillus versicolor, A. fumigatus, A. niger and Wallemia sebi. Secondary colonizers typically require a $a_{\mathrm{w}}$ between $0.80-0.90$ and are capable of thriving when the water activity or humidity changes throughout the day. This group is composed of various Alternaria, 
Cladosporium, Phoma and Ulocladium species. Tertiary colonizers or water damage molds require an $a_{\mathrm{w}}>0.90$ and include some of the more hazardous fungi found in damp buildings. Examples include Chaetomium globosum, Stachybotrys chartarum and Trichoderma species (Nielsen et al. 2003). The water activity of a specific building material will determine what mold species will grow indoors.

\section{Secondary Metabolites from $\boldsymbol{P}$. chrysogenum and $\boldsymbol{A}$. versicolor in the Indoor Environment}

The diversity of molds growing on damp or water damaged building materials and what building materials each of these fungi were associated with was recently investigated (Andersen et al. 2011). It was demonstrated that $P$. chrysogenum and $A$. versiocolor are the two most common fungal species found growing on damp building materials. C. globosum was the third most prevalent species found on water damaged building material where Acremonium strictum and Ulocladium spp. are also common. Secondary metabolites from indoor derived Ulocladium spp. and Acremonium spp. have not been detected suggesting a thorough investigation of indoor isolates of these two genera is required (Nielsen et al. 1999). Additionally, roquefortine C, sterigmatocystin and chaetoglobosin A have been directly detected in air, dust, fungal biomass and wallpaper samples (Polizzi et al. 2009). These are all reliably characterized toxic metabolites produced by $P$. chrysogenum, A. versiocolor and C. globosum. Due to their prevalence on damaged building materials, the secondary metabolite produced by $P$. chrysogenum and $A$. versiocolor derived from the indoor environment are reviewed here. Metabolites produced by $C$. globosum are discussed in chapter II. 
Species in the genus Penicillium were identified as the most frequently isolated $(\sim 70 \%)$ from water damaged building materials where $70-75 \%$ of the isolates studied were identified as $P$. chrysogenum (Andersen et al. 2011). Penicillium species are commonly isolated from insulation, wood, gypsum wallboard, manufactured wood, ceiling tiles and textiles (Miller et al. 2008). Nielsen et al. (1999) reported meleagrin and chrysogine from naturally and artificially infested damp building materials by $P$. chrysogenum however did not observe any roquefortine $\mathrm{C}$, a meleagrin precursor. A study of 109 houses in Wallaceburg, ON and twenty five culture collection isolates resulted in the phylogenetic examination of 198 P. chrysogenum indoor strains by Scott et al. (2004). This phylogenetic analysis revealed 4 clades of which there were 3 resolved lineages. Clade 4 was dominant making up more than $90 \%$ of the isolates examined. Clade 1 and 2 were shown to be sister lineages accounting for 5.6 and $1.0 \%$ of the isolates respectively whereas clade 3 accounted for $3.0 \%$. Clade 1, uncommonly from indoor dust relative to clade 4 , was isolated on raw wood and paper. Clade 4 isolates were obtained from paper, soil, construction materials and various indoor environments. It is also noteworthy that the strain isolated as a plate contaminant by Alexander Fleming in 1929 belongs to this lineage. De la Campa et al. (2007) examined the metabolite production of $P$. chrysogenum clade 1 and 4 where 13 strains were also studied by Scott et al. (2004) and the 17 other isolates were derived from Canadian indoor air samples. The geographically distributed strains examined were also dominated by clade $4(90 \%)$. The production of meleagrin, roquefortine $\mathrm{C}$, penicillin $\mathrm{G}$ and xanthocillin $\mathrm{X}$ was quantitatively examined however no chemotype differences were observed between the two Scott clades. Strains left too long in culture collections were poor producers of the mentioned compounds and one isolate derived from gypsum wallboard produced only penicillin G. Remaining strains appeared to differentially sequester penicillin $\mathrm{G}$ in the mycelia. 
Recently, Houbraken et al. (2011) examined an isolate from the Scott et al. (2004) study belonging to clade 2 . They were able to confirm the presence of meleagrin, andrastin A and B, chrysogine, a lumpidin related metabolite as well as secalonic acid D and F by HPLC-DAD. Phylogenetic analysis aimed at determining whether the two most common Scott clades within P. chrysogenum sensu lato were represented by one or two species was conducted. They demonstrated that clades 1 and 4 are indeed two different species, $P$. chrysogenum and $P$. rubens respectively. According to this investigation, Scott's lineages 1 and 2 are $P$. chrysogenum, clade 4 is $P$. rubens and clade 3 remains unknown at this time. Their examination of metabolites from $P$. rubens revealed most of the same metabolites as $P$. chrysogenum; however, it did not produce the lumpidin-related metabolite or either secalonic acids D and F. Scott et al. (2004) was unable to find $P$. rubens in outdoor air in the Wallaceburg, ON s. The abundance of $P$. rubens indoors opposed to the other 3 Scott clades suggests it has a competitive advantage within the indoor environment.

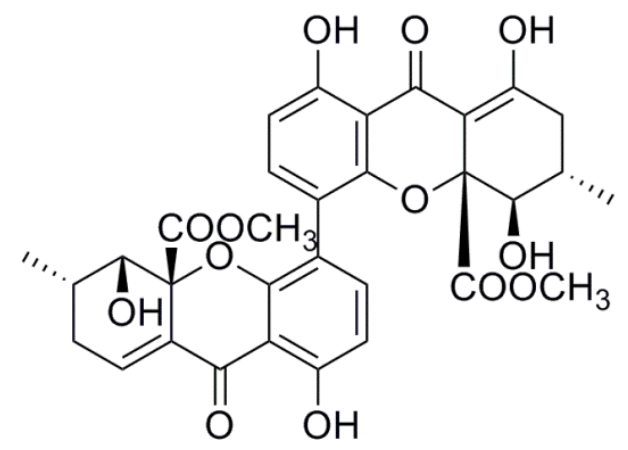<smiles>C=CC(C)(C)[C@]12C=C(O)C(=O)N(/C(=C/c3cnc[nH]3)C(=O)N1)[C@]21c2ccccc2N1OC</smiles><smiles>C[C@H](O)c1nc(=O)c2ccccc2[nH]1</smiles>

Figure 1.5: Structures of secalonic acid D, meleagrin and chrysogine isolated from either $P$. chrysogenum or P. rubens. 
Andersen et al. (2011) reports $A$. versiocolor as the most frequently identified mold species, $26.5 \%$ of all counts, from damp building materials; whereas, Nielsen et al. (1999) reports $P$. chrysogenum as the most common and $A$. versiocolor second. This species was evenly distributed on most damaged building materials however, was rarely found on wood. It is more commonly associated with some lower nutrient materials including concrete, vinyl, cork and glue while underrepresented on grout, wood, and plywood. A. versicolor is able to grow on a variety of artificially inoculated building materials examined but not on acoustic ceiling tiles (Nielsen et al. 1998). Besides growing on nutrient-poor substrates, it is a primary colonizer of building materials capable of proliferating on materials with a low $a_{w}$. Sterigmatocystin and 5methoxysterigmatocystin were detected on pine wood, wallpaper, gypsum board and chipboard inoculated with $A$. versicolor (Nielsen et al. 1998). All five strains examined produced these two toxins with the highest concentrations observed on wallpaper.

From these water damaged materials, up to approximately 7 and $20 \mu \mathrm{g} / \mathrm{cm}^{2}$ of 5 -methoxysterigmatocystin and sterigmatocystin, respectively were reported from A. versicolor. Typically, common indoor fungi produce relatively low amounts of secondary metabolites; however, sterigmatocystin may represent up to $1 \%$ of the $A$. versicolor biomass (Nielsen 1998). A. versicolor infested areas of non-sporulating red biomass yielded larger amounts of sterigmatocystin as opposed to the conidia (Nielsen et al. 1999). Tuomi et al. (2000) identified sterigmatocystin as the most prevalent toxin in mold damaged building materials. Of the sterigmatocystin-positive samples, $A$. versicolor was present in most of them. From eleven carpet dust samples, 49 of $50 \mathrm{~A}$. versicolor isolates were shown to produce sterigmatocystin. Further, from those eleven carpet dust samples, sterigmatocystin could be directly detected at up to $4 \mathrm{ng} / \mathrm{g}$ 
dust in two of the samples (Engelhart et al. 2002). Bloom et al. (2007) identified sterigmatocystin in 25 of 62 building material samples as well as one settled dust sample. The sterigmatocystin amounts in building material and settled dust samples were $110 \mathrm{pg} / \mathrm{mg}$ and $17 \mathrm{pg} / \mathrm{cm}^{2}$ respectively. A. versicolor was identified in the majority of sterigmatocystin positive samples.

In a follow up study by Bloom et al. (2009), 100 building materials, 18 settled dust and 37 cultured dust samples were collected over a one year period. The mycoflora and the amounts of selected potent mycotoxins were determined from the collected samples. The mean amounts of sterigmatocystin in building material samples was $7.1 \mathrm{ng} / \mathrm{mg}$, the third most frequent toxin observed; however was not observed in any of the settled dust samples. Most sterigmatocystinpositive samples were also positive for Aspergillus spp. Sterigmatocystin was less prevalent in the 2009 study compared to the one conducted in 2007; however, this is most likely a result of the different proportion of building materials sampled favoring growth of different molds.
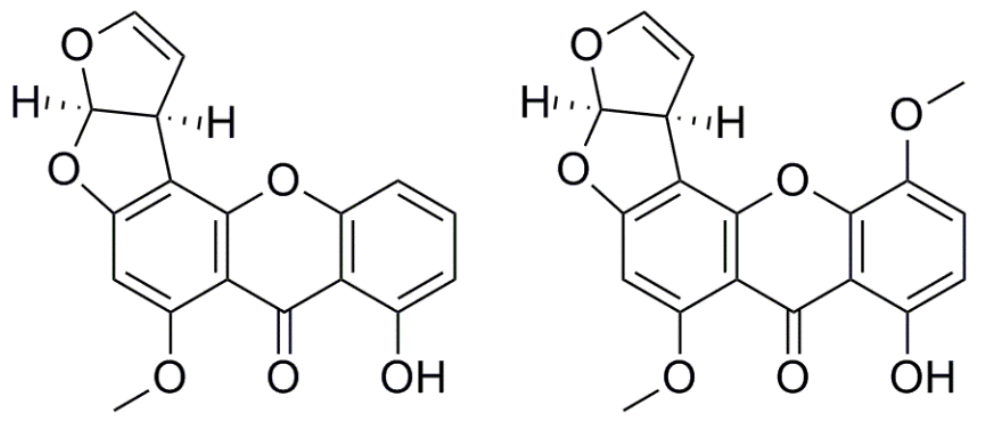

Figure 1.6: Structures of the carcinogenic metabolites, sterigmatocystin and 5methoxysterigmatocystin produced by $A$. versicolor. 


\section{PROJECT AIM}

It has been established that mold in damp buildings is associated with adverse human health consequences. The two primary effects observed in human population studies are increased upper respiratory disease and an allergic response in both atopic and non-atopic individuals. Allergy alone cannot explain this pattern of disease and secondary metabolites produced by fungi are now known to play a role in this disease process. At doses that could be experienced in buildings with mold problems, toxic secondary metabolites present on spores and mycelial fragments have been shown to affect lung biology in vivo. The goal of this investigation is to purify and structurally characterize the dominant toxic secondary metabolites produced by fungi commonly found growing in damp Canadian buildings. The secondary metabolite profiles of $C$. globosum, P. corylophilum, W. sebi and Trichoderma spp. obtained from the built environment will be investigated.

To determine how fungal secondary metabolites alter human lung biology in vivo requires the preparation of very pure metabolites for toxicity experiments (e.g. Miller et al. 2010; Rand et al. 2011; 2013). Purified metabolites will also be utilized as analytical standards to assess the toxigenic potential of strains of these common indoor species geographically representing Canada. The secondary metabolites isolated here will be purified and unambiguously characterized as opposed to being identified on a chromatogram which will aid mycologist when taking a polyphasic approach to identifying fungi. The existing literature concerning secondary metabolites from fungi is often convoluted due to dubious analytical methods or incorrect fungal 
identifications. Secondary metabolite profiles in polyphasic taxonomic studies are often utilized when classifying or identifying fungal species as they are typically very species specific.

Fermentation conditions that favor metabolite production of each species will be determined. Metabolite screening of crude filtrate extracts from each strain using the fermentation conditions elucidated will be achieved by HPLC-DAD or LC-UV-MS. Large scale fermentations of representative strains of each species will be performed to generate adequate amounts of metabolites for structural elucidation, toxicity assays and determination of various strains toxigenic potential. Major metabolites can be purified by a combination of normal phase column chromatography, Sephadex, SPE, prep-TLC or preparative reverse phase HPLC. Unambiguous structural characterization of purified metabolites will be achieved by high resolution mass spectrometry, nuclear magnetic resonance (NMR) spectroscopy, chemical derivatization, other spectroscopic methods (UV, OR, CD) and evaluation of the existing literature. 


\section{CHAPTER II}

ISOLATION, CHARACTERIZATION AND QUANTIFICATION OF CHAETOGLOBOSINS AND AZAPHILONES FROM CHAETOMIUM GLOBOSUM 


\section{INTRODUCTION}

Chaetomium globosum is one of the most common fungi found growing on wet or damp building materials in Canada and Europe (Flannigan and Miller 2011; Fogle et al. 2007; Miller et al. 2008). Andersen et al. (2011) list it as the third most common fungal species on damp building materials. This species, the most common species within the genus, is distributed worldwide, commonly found in soil and plant debris due to its strong cellulolytic activity. When the cellulolytic activity of several fungi was tested, $C$. globosum readily degraded cotton fabrics (Flannigan and Miller 2001). Chaetomium species are tertiary colonizers that require a $a_{w}>0.90$ to proliferate (Nielsen et al. 1999). It is common on wallboard, solid wood, textiles, manufactured wood, ceiling tiles and is frequently found on insulation (Flannigan and Miller 2011; Miller et al. 2008). C. globosum is also tolerant to calcium salts since it is regularly isolated from gypsum wallboard, a material that is primarily made up of these salts (Miller et al. 2008). As a hydrophilic mold, it is not surprising that this prominent indoor species is abundant in bathrooms, kitchens and around window frames (Piecková 2003). C. globosum has an optimum temperature for growth between $16-25^{\circ} \mathrm{C}$, will not grow above $37{ }^{\circ} \mathrm{C}$ and prefers a neutral pH (Fogle et al. 2008). It also has a characteristic appearance; distinct black or dark green parathecia, visible to the naked eye (Nielsen et al. 1999).

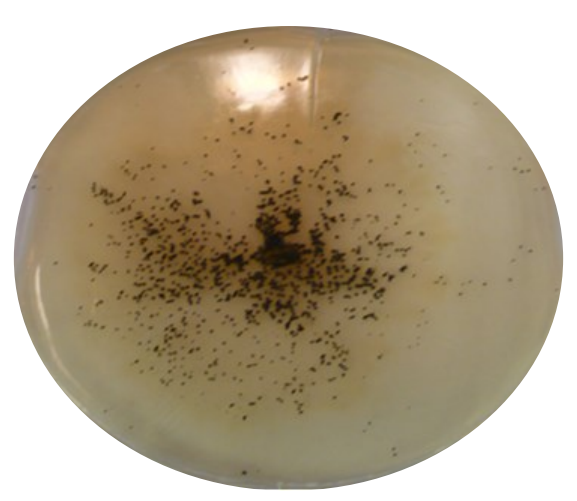

Figure 2.1: C. globosum grown on 2\% MEA. 
Previous studies of metabolites from C. globosum in Canada were done in an agricultural context, namely on identifying the causative agents of ill-thrift in sheep before 1973. Isolates obtained from pastures and moldy corn in Nova Scotia established that chetomin is at least partially responsible for the etiology of the ill-thrift (Brewer et al. 1972). Chetomin is moderately toxic to rats and ewes, exhibits strong antibacterial properties (Brewer et al. 1972) and is a potent inhibitor of HeLa and cultured epithelial cells (Trown 1968). These strains also produced the purple pigment cochliodinol that is moderately antifungal and antibiotic (Brewer et al. 1968). Cochliodinol was not cytotoxic to mammalian cells, nor did it demonstrate any toxicity in turkeys or rats (Brewer et al. 1970). The structures of chetomin and cochliodinol are shown in figure 2.2 .

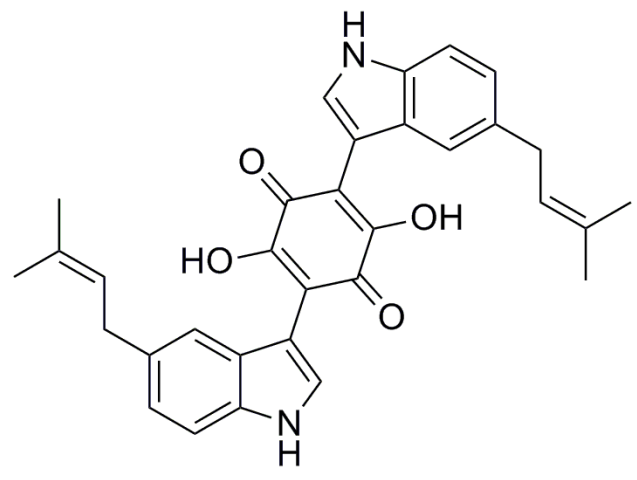

Cochliodinol

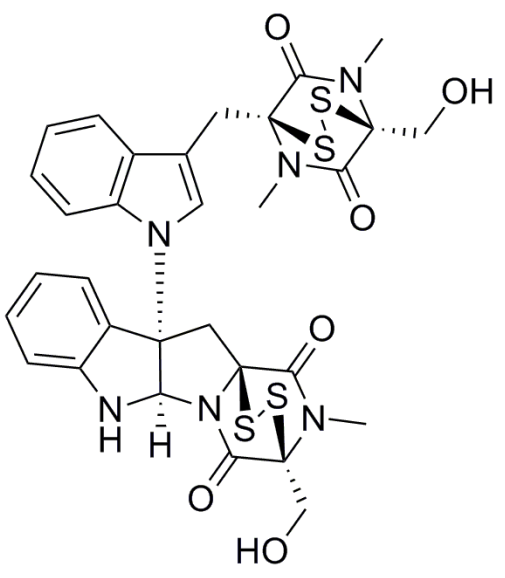

Chetomin

Figure 2.2: Structures of the toxin chetomin and the purple pigment cochliodinol.

C. globosum produces a variety of cytotoxic cytochalasans called chaetoglobosins. Chaetoglobosins A-B (Sekita et al. 1973), C-F (Sekita et al. 1976), G and J (Sekita et al. 1977), Q, R, and T (Jiao et al. 2004) have all been isolated from soil-derived C. globosum strains. The metabolites arise from a mixed polyketide-amino acid biosynthetic pathway and have a wide 
variety of biological activities (Scherlach et al. 2010). Chaetoglobosins all possess the amino acid tryptophan and a structurally variable thirteen-membered macrocycle. Other cytochalasans may bear the amino acid phenylalanine as opposed to tryptophan (Sekita et al. 1976).

Chaetoglobosins inhibits cytoplasmic cleavage and restricting movement in mammalian cells. This is achieved by capping actin, specifically within in the hydrophobic cleft between actin subunits 1 and 3, inhibiting the actin cytoskeletal proteins from polymerizing into microfilaments (Scherlach et al. 2010).

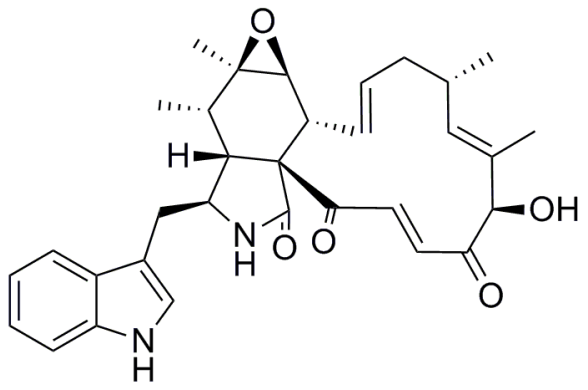

Chaetoglobosin A

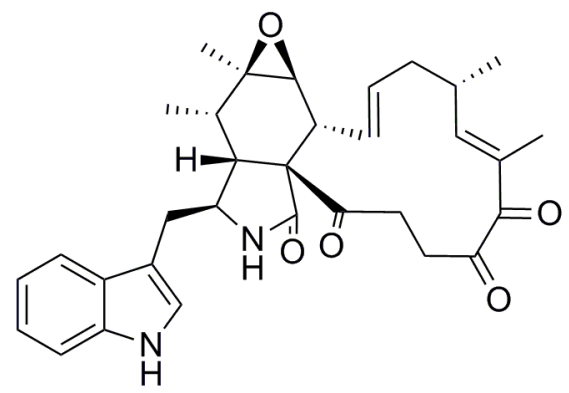

Chaetoglobosin C

Figure 2.3: Structures of chaetoglobosin A and C.

Chaetoglobosins are potently cytotoxic metabolites which cause polynucleation of HeLa cells and acute oral toxicity to experimental animals (Udagawa et al. 1978; Umeda et al. 1975). Cytotoxic effects are observed for chaetoglobosin A and $\mathrm{C}$ at $3.2-10 \mu \mathrm{g} / \mathrm{mL}$ and $10-32 \mu \mathrm{g} / \mathrm{mL}$ in HeLa cells, respectively (figure 2.3; Udagawa et al. 1978). However, the toxic effects elicited by chaetoglobosins depend on the mechanism of exposure which is lower when ingested orally compared to intravenous injection (Nielsen et al. 1999). LD $_{50}$ values for subcutaneous injections in mice were determined to be $6.5 \mathrm{mg} / \mathrm{kg}$ bw and $17.8 \mathrm{mg} / \mathrm{kg}$ bw for male and females respectively (Binder and Tamm 1973). Chaetoglobosin A has low toxicity to mice (Ohtsubo et 
al. 1978) but is potently antifungal (Amemiya et al. 1994; Betina et al. 1972), antibacterial and cytotoxic (Betina et al. 1972; Scherlach et al. 2010; Umeda et al. 1975).

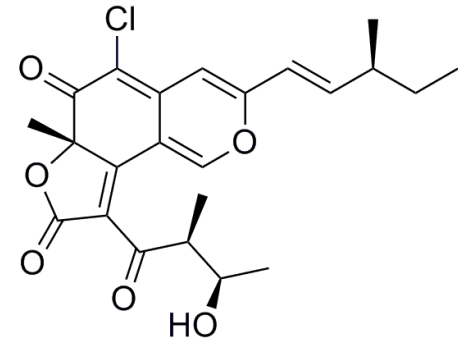

Chaetoviridin A

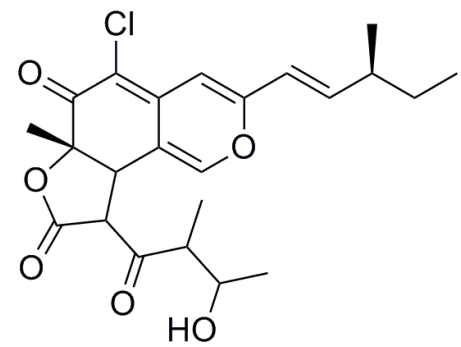

Chaetoviridin C<smiles>CC[C@H](C)/C=C/C1=CC2=C(Cl)C(=O)[C@@]3(C)OC(O)(C(C)C(C)O)C(C(=O)O)=C3C2=CO1</smiles>

Chaetoviridin B

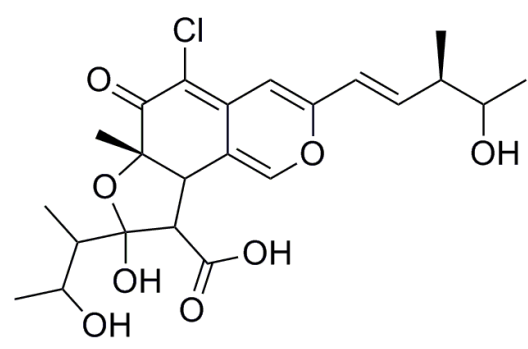

Chaetoviridin D

Figure 2.4: Structures of chaetoviridin A-D isolated from C. globosum.

The azaphilones chaetoviridin A-D (figure 2.4) were additionally isolated from a soil $C$. globosum isolate (Takahashi et al. 1990). Azaphilones are a structurally diverse group of highly conjugated polyketides that possess a pyrano-quinone core structure. They have been isolated from more than 23 fungal genera and have a variety of intriguing biological activities (Osmanova et al. 2010). Azaphilones isolated from C. globosum include chaetoviridins as well as chaetomugilins, chaetoglobins, and isochromophilones (Ming et al. 2008; McMullin et al. 2013). Chaetoviridins demonstrate weak monoamine oxidase inhibition (Takahashi et al. 1990), potent antifungal activity (Park et al. 2005) and inhibit cholesteryl ester transferase (Tomoda et al. 1999). Chaetomugilin metabolites have been shown to exhibit cytotoxic growth inhibition 
activity against murine P388, human HL-60, murine L1210 and human KB cell lines (Muroga et al. 2009). Isochromophilones inhibit acyl-CoA acytltransferase (Arai et al. 1995). However, the non-selective cytotoxicity most azaphilones elicit prevents them from being pursued as useful pharmaceutical targets (Osmanova et al. 2010).

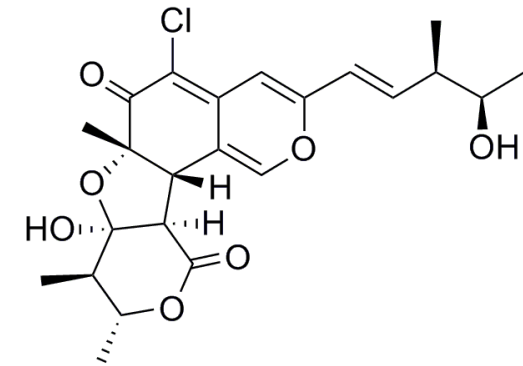

Chaetomugilin A

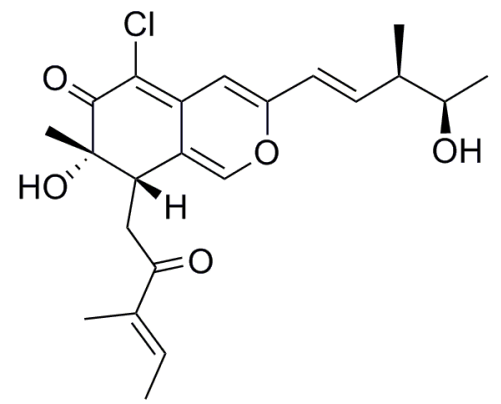

Chaetomugilin I

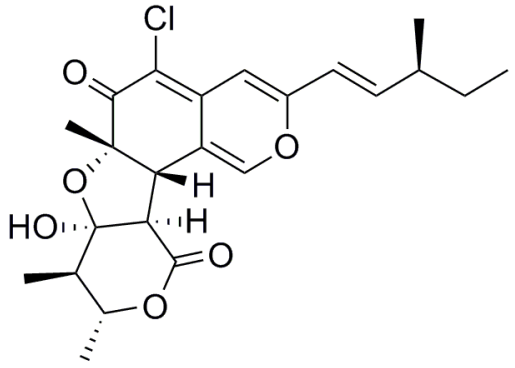

Chaetomugilin D

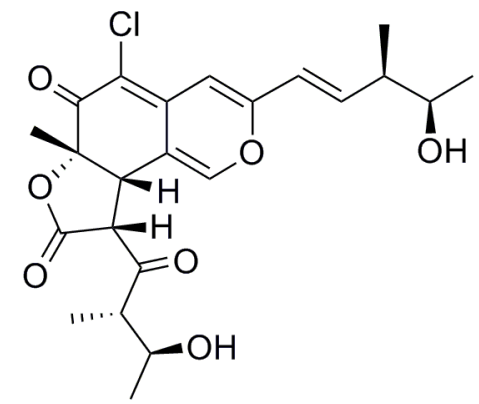

Chaetomugilin M

Figure 2.5: Structures of chaetomugilin A, D, I and M isolated from C. globosum.

Examination of C. globosum strains reported from marine environments or as endophytes has yielded chaetomugilins A-F (Yasuhide et al. 2008), I-O (Muroga et al. 2009) and secochaetomugilins A-D (Yamada et al. 2008) from the marine fish Mugil cephalus, the indole alkaloid chaetoglobosin U (Ding et al. 2006) and chaetoglobins A and B (Ming et al. 2008) from the normal stem of Imperata cylindrical, the benzaldehyde derivative chaetopyranin from the marine red algae Polysiphonia urceolata (Wang et al. 2006), globosumones A-C from the stem 
tissue of Ephedra fasiculata (Bharat et al. 2005) and various azaphilones from the leaves of a Viguiera robusta endophytic strain (Borges et al. 2011). The structures of chaetoglobosin U and chaetoglobin A characterized from endophytic C. globosum isolates can be found in figure 2.6. Chaetoglobins A and B inhibited the breast cancer cell line MCF-7 as well as the colon cancer cell line SW1116 with $\mathrm{IC}_{50}$ values of 26.8 and $35.4 \mu \mathrm{g} \mathrm{mL}^{-1}$, respectively (Ming et al. 2008).

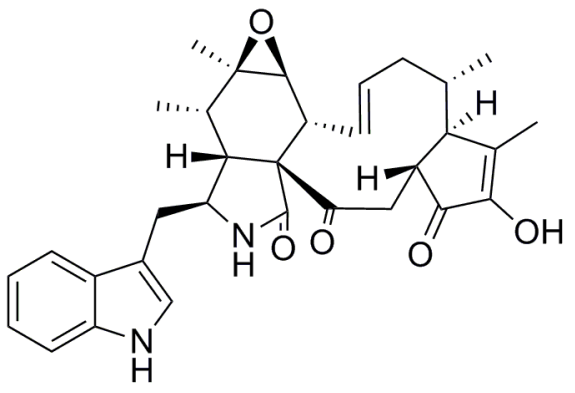

Chaetoglobosin $U$<smiles>CC(=O)O[C@H]1C(=O)C2=CNC(CCC[C@H](C)O)=CC2=C(C2=C3C=C(CCC[C@H](C)O)NC=C3C(=O)[C@](C)(OC(C)=O)C2=O)C1=O</smiles>

Chaetoglobin A

Figure 2.6: Structures of chaetoglobosin $U$ and chaetoglobin A.

Chaetoglobosin A and C, were shown to be produced on various naturally and artificially infested damp building materials in the US and Denmark (Fogle et al. 2007; Nielsen et al. 1999). Numerous unidentified C. globosum metabolites were additionally reported to be produced on damp wallpaper by Nielsen et al. (1999). More recently, chaetoglobosin A and chetomin were reported from a moldy wooden wall scraping from Vienna (Vishwanath et al. 2009) and chaetoglobosin A was additionally detected from moldy building materials in Finland (Täubel et al. 2011). Strains were collected from the built environment in Canada and a comprehensive examination of the secondary metabolite profiles of Canadian strains derived from damp buildings was conducted herein. 


\section{RESULTS AND DISCUSSION}

In order to structurally characterize the major metabolites from the strains of $C$. globosum obtained, large scale fermentations of a representative strain, DAOM 240349, were carried out. The crude EtOAc extract was initially separated by column chromatography and the major components were further purified by reverse phase semi-preparative HPLC. Each major metabolite was structurally characterized by high resolution mass spectrometry, NMR, UV, OR and comparisons to the literature. This work was highlighted by the characterization of several previously reported chaetoglobosins and azaphilones as well as three new azaphilones. Major metabolites isolated were utilized as analytical standards to quantify each compound from the twenty five indoor C. globosum isolates within the labs possession. No evidence of chemotypes was observed in the strains investigated here, refer to table 2.4. Each strain obtained from Canadian buildings was characterized by morphology, sequencing of their internal transcribed spacer (ITS), and secondary metabolite profile (see materials and methods).

\section{Major Metabolites: Chaetoglobosins and Azaphilones}

Chaetoglobosin A (2.1); pale yellow solid; $[\alpha]_{\mathrm{D}}-269$ (c 0.2, MeOH) [-270 (MeOH), Sekita et al. 1973]; UV (MeOH)/nm $\lambda_{\max }(\log \varepsilon) 280$ and (3.51) 220 (3.52); HRESIMS $m / z$ 529.2707 [M+H] ${ }^{+}$ (calculated for $\left.\mathrm{C}_{32} \mathrm{H}_{37} \mathrm{~N}_{2} \mathrm{O}_{5}, 529.2702\right) ;{ }^{1}$ Hand ${ }^{13} \mathrm{C}$ NMR data were consistent with published data (Cole and Schweikert 2003) and are reported in table 2.1. The presence of chaetoglobosin A in the filtrate extracts of C. globosum strains examined was confirmed by an analytical standard (Alexis Biochemicals). The HRESIMS spectrum for chaetoglobosin A can be found in figure 2.12. The structure of chaetoglobosin A can be found in figure 2.7. 


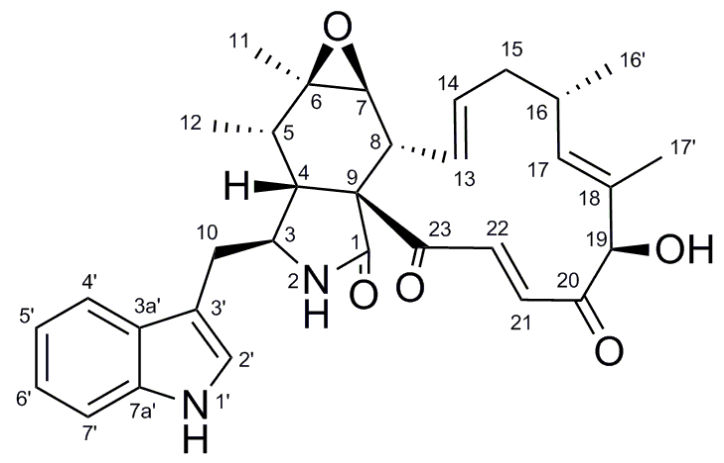

Figure 2.7: Structure of chaetoglobosin A (2.1).

Chaetoglobosin F (2.2); pale yellow solid; $[\alpha]_{\mathrm{D}}-42\left(\mathrm{c} 0.4, \mathrm{CHCl}_{3}\right)\left[-69\left(\mathrm{CHCl}_{3}\right)\right.$, Sekita et al. 1976]; UV (MeOH)/nm $\lambda_{\max } 290$ (3.44), 280 (3.50), and 220 (3.74); HRESIMS $m / z 531.2865$ $[\mathrm{M}+\mathrm{H}]^{+}$(calculated for $\mathrm{C}_{32} \mathrm{H}_{39} \mathrm{~N}_{2} \mathrm{O}_{5}, 531.2859$ ); ${ }^{1} \mathrm{Hand}{ }^{13} \mathrm{C}$ NMR data were consistent with published data (Cole and Schweikert 2003) and are reported in table 2.1. The structure of chaetoglobosin F can be found in figure 2.8 .

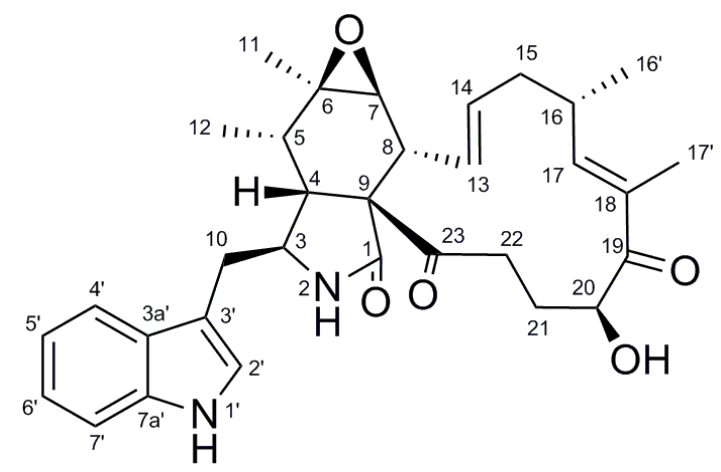

Figure 2.8: Structure of chaetoglobosin F (2.4).

Chaetoglobosin C (2.3); colorless solid; $[\alpha]_{\mathrm{D}}-40(\mathrm{c} 0.05, \mathrm{MeOH})[-30(\mathrm{MeOH})$, Sekita et al. 1976]; $\mathrm{UV}(\mathrm{MeOH}) / \mathrm{nm} \lambda_{\max } 280$ (2.66) and 220 (3.05); HRESIMS m/z 529.2706 [M+H] $]^{+}$ (calculated for $\mathrm{C}_{32} \mathrm{H}_{37} \mathrm{~N}_{2} \mathrm{O}_{5}, 529.2702$ ); ${ }^{1} \mathrm{H}$ data were consistent with published data (Cole and Schweikert 2003; Sekita et al. 1976) and are reported in table 2.1. The presence of 
chaetoglobosin $\mathrm{C}$ in the extracts of $C$. globosum was confirmed by an analytical standard (Alexis Biochemicals). The ${ }^{13} \mathrm{C}$ data for $\mathbf{2 . 3}$ are adapted from Cole and Schweikert (2003). The structure of chaetoglobosin $\mathrm{C}$ can be found in figure 2.9.

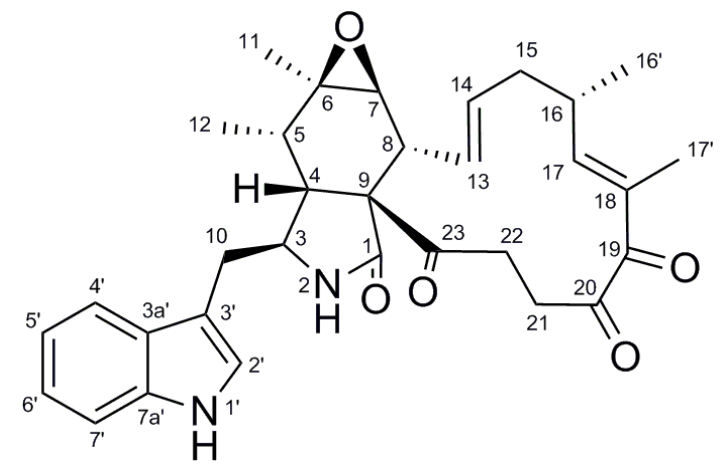

Figure 2.9: Structure of chaetoglobosin C (2.3).

Chaetomugilin D (2.4); optically active yellow gum; $[\alpha]_{\mathrm{D}}-89$ (c $\left.0.1, \mathrm{MeOH}\right)[-170$ (c 0.1 , EtOH), Yasuhide et al. 2008]; UV (MeOH)/nm $\lambda \max (\log \varepsilon) 385$ (3.10), 290 (2.90) and 220 (3.03); HRESIMS $m / z 435.1600[\mathrm{M}+\mathrm{H}]^{+}$(calculated for $\mathrm{C}_{23} \mathrm{H}_{28} \mathrm{O}_{6} \mathrm{Cl}, 435.1574$ ); ${ }^{1} \mathrm{H}$ and ${ }^{13} \mathrm{C}$ NMR data were consistent with published data (Yasuhide et al. 2008) and are reported in table 2.2. The approximate $3: 1$ peak ratio of $[\mathrm{M}+\mathrm{H}]^{+}:[\mathrm{M}+\mathrm{H}+2]^{+}$indicates the presence of a single chlorine atom in the molecule, figure 2.12. The structure of chaetomugilin D can be found in figure 2.10 . 
Table 2.1: ${ }^{1} \mathrm{H}(400 \mathrm{MHz})$ and ${ }^{13} \mathrm{C}$ NMR $(100 \mathrm{MHz})$ data for chaetoglobosin A (2.1), F (2.2) and C (2.3).

\begin{tabular}{|c|c|c|c|c|c|c|}
\hline \multirow[b]{2}{*}{ Position } & \multicolumn{2}{|r|}{$(2.1), \mathrm{CD}_{3} \mathrm{CN}$} & \multicolumn{2}{|r|}{ (2.2), $\mathrm{CDCl}_{3}$} & \multicolumn{2}{|r|}{ (2.3), $\mathrm{CD}_{3} \mathrm{OD}$} \\
\hline & $\delta_{\mathrm{C},}$ type & $\delta_{\mathrm{H}}(J, \mathrm{~Hz})$ & $\delta_{\mathrm{C}}$ type & $\delta_{\mathrm{H}}(J, \mathrm{~Hz})$ & $\delta_{\mathrm{C},}$ type & $\delta_{\mathrm{H}}(J, \mathrm{~Hz})$ \\
\hline 1 & $173.9, \mathrm{C}$ & & $174.8, \mathrm{C}$ & & $173.8, \mathrm{C}$ & \\
\hline 2 & & $6.65, \mathrm{~s}$ & & $6.08, \mathrm{~s}$ & & \\
\hline 3 & $53.2, \mathrm{CH}$ & $3.80, \mathrm{~m}$ & $52.5, \mathrm{CH}$ & $3.75, \mathrm{~m}$ & $52.3, \mathrm{CH}$ & $3.80, \mathrm{~m}$ \\
\hline 4 & $47.5, \mathrm{CH}$ & $2.73, \mathrm{o}$ & $49.4, \mathrm{CH}$ & $2.66, \mathrm{~m}$ & $48.4, \mathrm{CH}$ & $2.82, \mathrm{~m}$ \\
\hline 5 & $37.1, \mathrm{CH}$ & $1.71, \mathrm{dq}(5.5,7.2)$ & $36.4, \mathrm{CH}$ & $1.80, \mathrm{~m}$ & $36.1, \mathrm{CH}$ & $1.82, \mathrm{~m}$ \\
\hline 6 & $58.4, \mathrm{C}$ & & $57.4, \mathrm{C}$ & & $56.6, \mathrm{C}$ & \\
\hline 7 & $62.7, \mathrm{CH}$ & $2.70, \mathrm{~d}(5.2)$ & $61.8, \mathrm{CH}$ & $2.82, \mathrm{~d}(5.7)$ & $60.3, \mathrm{CH}$ & $2.68, \mathrm{~d}(5.7)$ \\
\hline 8 & $48.7, \mathrm{CH}$ & $2.08, \mathrm{dd}(5.2,9.7)$ & $48.4, \mathrm{CH}$ & $2.26, \mathrm{dd}(5.7,10.0)$ & $48.4, \mathrm{CH}$ & $2.26(5.6,10.0)$ \\
\hline 9 & $64.0, \mathrm{C}$ & & $64.5, \mathrm{C}$ & & $62.2, \mathrm{C}$ & \\
\hline 10 & $33.5, \mathrm{CH}_{2}$ & $2.73, \mathrm{o}$ & $34.5, \mathrm{CH}_{2}$ & $2.64, \mathrm{~m}$ & $39.5, \mathrm{CH}_{2}$ & $2.68, \mathrm{~m}$ \\
\hline 11 & $13.1, \mathrm{CH}_{3}$ & $1.19, \mathrm{~s}$ & $12.8, \mathrm{CH}_{3}$ & $1.19, \mathrm{~s}$ & 9.9, $\mathrm{CH}_{3}$ & $1.24, \mathrm{~s}$ \\
\hline 12 & $19.6, \mathrm{CH}_{3}$ & $0.97, \mathrm{~d}(7.2)$ & $19.5, \mathrm{CH}_{3}$ & $1.11, \mathrm{~d}(7.2)$ & $19.3, \mathrm{CH}_{3}$ & $1.11, \mathrm{~d}(7.3)$ \\
\hline 13 & $129.0, \mathrm{CH}$ & 6.05, ddd $(1.8,9.6,15.5)$ & $128.7, \mathrm{CH}$ & 6.35, ddd $(2.0,9.8,15.2)$ & $127.0, \mathrm{CH}$ & $6.06, \mathrm{dd}(10.1,15.4)$ \\
\hline 14 & $134.2, \mathrm{CH}$ & 5.17, ddd $(3.6,10.2,15.5)$ & $133.5, \mathrm{CH}$ & $5.24, \mathrm{~m}$ & $133.1, \mathrm{CH}$ & $5.03, \mathrm{~m}$ \\
\hline \multirow[t]{2}{*}{15} & $41.8, \mathrm{CH}_{2}$ & $1.93, \mathrm{~m}$ & $41.1, \mathrm{CH}_{2}$ & $2.05, \mathrm{~m}$ & $32.6, \mathrm{CH}_{2}$ & \\
\hline & & $2.22, \mathrm{~m}$ & & $2.40, \mathrm{~m}$ & & \\
\hline 16 & $32.8, \mathrm{CH}$ & $2.48, \mathrm{~m}$ & $33.3, \mathrm{CH}$ & $2.74, \mathrm{~m}$ & $37.0, \mathrm{CH}$ & $2.72, \mathrm{~m}$ \\
\hline 17 & $140.2, \mathrm{CH}$ & $5.45, \mathrm{dd}(1.7,9.5)$ & $149.5, \mathrm{CH}$ & $6.11, \mathrm{~d}(9.0)$ & $155.8, \mathrm{CH}$ & $5.92, \mathrm{~d}(9.6)$ \\
\hline 18 & $130.0, \mathrm{C}$ & & $127.0, \mathrm{C}$ & & $130.9, \mathrm{C}$ & \\
\hline 19 & $82.7, \mathrm{CH}$ & $4.88, \mathrm{~s}$ & $203.5, \mathrm{C}$ & & $196.1, \mathrm{C}$ & \\
\hline 20 & 201.8, C & & $71.8, \mathrm{CH}$ & $4.68, \mathrm{t}(4.7)$ & $205.2, \mathrm{C}$ & \\
\hline \multirow[t]{2}{*}{21} & $133.5, \mathrm{CH}$ & $6.35, \mathrm{~d}(16.5)$ & $31.5, \mathrm{CH}_{2}$ & $1.70, \mathrm{~m}$ & $31.9, \mathrm{CH}_{2}$ & $2.22, \mathrm{~m}$ \\
\hline & & & & $1.82, \mathrm{~m}$ & & $2.40, \mathrm{~m}$ \\
\hline \multirow[t]{2}{*}{22} & $136.3, \mathrm{CH}$ & $7.40, \mathrm{~d}(16.5)$ & $38.1, \mathrm{CH}_{2}$ & $2.44, \mathrm{~m}$ & $31.9, \mathrm{CH}_{2}$ & $2.44, \mathrm{~m}$ \\
\hline & & & & $2.88, \mathrm{~m}$ & & $2.81, \mathrm{dd}(3.54,15.5)$ \\
\hline 23 & $198.9, \mathrm{C}$ & & 208.3, C & & 208.1, C & \\
\hline 1 ' & & $9.15, \mathrm{~s}$ & & $8.46, \mathrm{~s}$ & & \\
\hline 2 & $125.1, \mathrm{CH}$ & $6.98, \mathrm{~d}(2.4)$ & $123.3, \mathrm{CH}$ & $6.98, \mathrm{~d}(2.2)$ & $125.1, \mathrm{CH}$ & 7.02, s \\
\hline 3 , & $110.4, \mathrm{C}$ & & $110.4, \mathrm{C}$ & & $108.0, \mathrm{C}$ & \\
\hline 4 ' & $119.3, \mathrm{CH}$ & $7.49, \mathrm{~d}(7.8)$ & $118.2, \mathrm{CH}$ & $7.49, \mathrm{~d}(7.8)$ & $118.3, \mathrm{CH}$ & $7.54, \mathrm{~d}(8.0)$ \\
\hline 5, & $119.9, \mathrm{CH}$ & $7.02, \mathrm{~m}$ & $119.9, \mathrm{CH}$ & $7.14, \mathrm{~m}$ & $118.6, \mathrm{CH}$ & $7.06, \mathrm{~m}$ \\
\hline 6 ' & $122.3, \mathrm{CH}$ & $7.07, \mathrm{~m}$ & $122.4, \mathrm{CH}$ & $7.20, \mathrm{~m}$ & $120.9, \mathrm{CH}$ & $7.12, \mathrm{~m}$ \\
\hline 7 & $112.3, \mathrm{CH}$ & $7.33, \mathrm{~d}(8.0)$ & $111.5, \mathrm{CH}$ & $7.37, \mathrm{~d}(8.1)$ & $111.3, \mathrm{CH}$ & $7.33, \mathrm{~d}(8.1)$ \\
\hline 16 ' & $21.1, \mathrm{CH}_{3}$ & $0.95, \mathrm{~d}(6.4)$ & $19.9, \mathrm{CH}_{3}$ & $1.02, \mathrm{~d}(6.5)$ & $19.1, \mathrm{CH}_{3}$ & $0.99, \mathrm{~d}(6.63)$ \\
\hline 18 ' & $10.7, \mathrm{CH}_{3}$ & $1.28, \mathrm{~s}$ & $12.2, \mathrm{CH}_{3}$ & $1.81, \mathrm{~s}$ & $12.3, \mathrm{CH}_{3}$ & $1.74, \mathrm{~s}$ \\
\hline $3 \mathbf{a}^{\prime}$ & $128.6, \mathrm{C}$ & & $134.2, \mathrm{C}$ & & 127.6, C & \\
\hline $7 a^{\prime}$ & $137.3, \mathrm{C}$ & & $136.2, \mathrm{C}$ & & $135.8, \mathrm{C}$ & \\
\hline
\end{tabular}




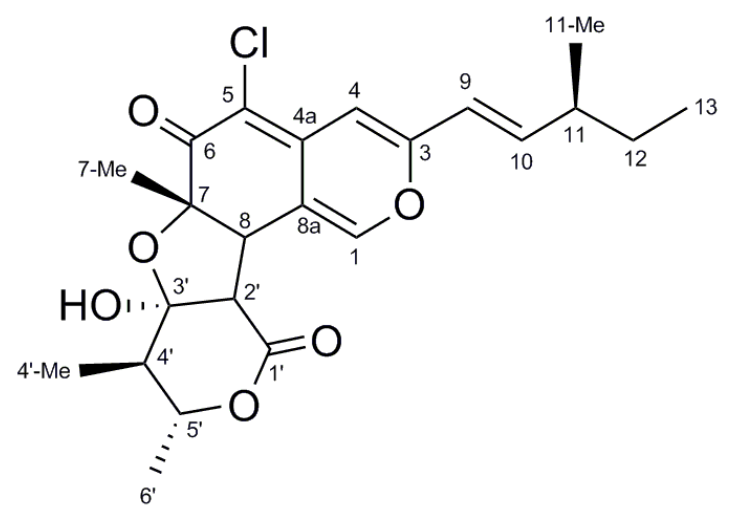

Figure 2.10: Structure of chaetomugilin D (2.4).

Table 2.2: ${ }^{1} \mathrm{H}(400 \mathrm{MHz})$ and ${ }^{13} \mathrm{C}$ NMR $(100 \mathrm{MHz})$ data for chaetomugilin $\mathrm{D}(\mathbf{2 . 4})$ in $\mathrm{CD}_{3} \mathrm{CN}$.

\begin{tabular}{|c|c|c|}
\hline \multirow[b]{2}{*}{ Position } & \multicolumn{2}{|c|}{$(2.4)$} \\
\hline & $\delta_{\mathrm{C}}$ type & $\delta_{\mathrm{H}}(J, \mathrm{~Hz})$ \\
\hline 1 & $146.3, \mathrm{CH}$ & $7.30, \mathrm{~s}$ \\
\hline 3 & $158.3, \mathrm{C}$ & \\
\hline 4 & $105.4, \mathrm{CH}$ & $6.60, \mathrm{~s}$ \\
\hline $4 a$ & $140.7, \mathrm{C}$ & \\
\hline 5 & $110.8, \mathrm{C}$ & \\
\hline 6 & 188.9, C & \\
\hline 7 & $84.1, \mathrm{C}$ & \\
\hline 8 & $50.1, \mathrm{CH}$ & $3.20, \mathrm{~d}(10.5)$ \\
\hline $8 \mathbf{a}$ & $115.3, \mathrm{C}$ & \\
\hline 9 & $121.2, \mathrm{CH}$ & $6.22, \mathrm{~d}(15.8)$ \\
\hline 10 & $146.6, \mathrm{CH}$ & $6.54, \mathrm{dd}(8.0,15.8)$ \\
\hline 11 & $39.3, \mathrm{CH}$ & $2.27, \mathrm{~m}$ \\
\hline 12 & $29.7, \mathrm{CH}_{2}$ & $1.43, \mathrm{~m}$ \\
\hline 13 & $11.9, \mathrm{CH}_{3}$ & $0.89, \mathrm{t}(7.4)$ \\
\hline 7- $\mathrm{CH}_{3}$ & $23.2, \mathrm{CH}_{3}$ & $1.29, \mathrm{~s}$ \\
\hline $11-\mathrm{CH}_{3}$ & $19.5, \mathrm{CH}_{3}$ & $1.06, \mathrm{~d}(6.7)$ \\
\hline 1 ' & $170.7, \mathrm{C}$ & \\
\hline 2', & $59.1, \mathrm{CH}$ & $2.88, \mathrm{~d}(10.5)$ \\
\hline 3, & 104.7, C & \\
\hline 4 , & $46.1, \mathrm{CH}$ & $1.80, \mathrm{dq}(6.4,10.2)$ \\
\hline 5 , & $77.2, \mathrm{CH}$ & 4.33 , dq $(6.9,10.2)$ \\
\hline 6 ' & $18.6, \mathrm{CH}_{3}$ & $1.32, \mathrm{~d}(6.9)$ \\
\hline $4^{\prime}-\mathrm{CH}_{3}$ & $8.9, \mathrm{CH}_{3}$ & $1.04, \mathrm{~d}(6.4)$ \\
\hline
\end{tabular}

Chaetoviridin A (2.5); optically active yellow gum; $[\alpha]_{\mathrm{D}}+80$ (c $\left.0.01, \mathrm{MeOH}\right)[+98(\mathrm{c} 0.05$, $\mathrm{CHCl}_{3}$ ), Takahashi et al. 1990]; UV (MeOH)/nm $\lambda_{\max }(\log \varepsilon) 370$ (3.81) and 320 (3.81); HRESIMS $m / z 433.1437[\mathrm{M}+\mathrm{H}]^{+}$(calculated for $\mathrm{C}_{23} \mathrm{H}_{26} \mathrm{O}_{6} \mathrm{Cl}, 433.1418$ ); ${ }^{1} \mathrm{H}$ and ${ }^{13} \mathrm{C}$ NMR data 
was consistent with published data (Takahashi et al. 1990) and are reported in table 2.3. An approximate $3: 1$ peak ratio of $[\mathrm{M}+\mathrm{H}]^{+}:[\mathrm{M}+\mathrm{H}+2]^{+}$was also observed indicating the presence of a single chlorine atom in the molecule. The structure of chaetoviridin A can be found in figure 2.11.

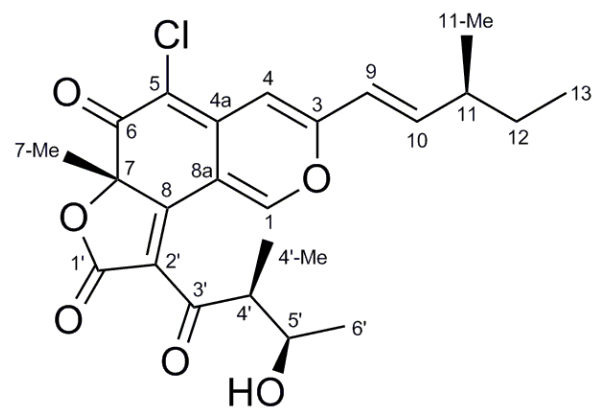

Figure 2.11: Structure of chaetoviridin A (2.5).

Table 2.3: ${ }^{1} \mathrm{H}(400 \mathrm{MHz})$ and ${ }^{13} \mathrm{C}$ NMR $(100 \mathrm{MHz})$ data for chaetoviridin $\mathrm{A}(\mathbf{2 . 5})$ in $\mathrm{CD}_{3} \mathrm{CN}$.

\begin{tabular}{|c|c|c|}
\hline \multirow[b]{2}{*}{ Position } & \multicolumn{2}{|c|}{$(2.5)$} \\
\hline & $\delta_{\mathrm{C}, \text { type }}$ & $\delta_{\mathrm{H}}(J, \mathrm{~Hz})$ \\
\hline 1 & $152.0, \mathrm{CH}$ & $8.59, \mathrm{~s}$ \\
\hline 3 & $158.2, \mathrm{C}$ & \\
\hline 4 & $105.9, \mathrm{CH}$ & $6.66, \mathrm{~s}$ \\
\hline $4 a$ & $141.4, \mathrm{C}$ & \\
\hline 5 & 108.7, C & \\
\hline 6 & 184.3, C & \\
\hline 7 & $88.3, \mathrm{C}$ & \\
\hline 8 & $162.2, \mathrm{C}$ & \\
\hline $8 \mathbf{a}$ & $111.3, \mathrm{C}$ & \\
\hline 9 & $120.8, \mathrm{CH}$ & $6.25, \mathrm{~d}(15.4)$ \\
\hline 10 & $148.2, \mathrm{CH}$ & $6.65, \mathrm{dd}(7.1,15.6)$ \\
\hline 11 & $39.5, \mathrm{CH}$ & $2.29, \mathrm{~m}$ \\
\hline 12 & 29.6, $\mathrm{CH}_{2}$ & $1.43, \mathrm{~m}$ \\
\hline 13 & $11.9, \mathrm{CH}_{3}$ & $0.89, \mathrm{t}(7.4)$ \\
\hline 7- $\mathrm{CH}_{3}$ & $25.7, \mathrm{CH}_{3}$ & $1.63, \mathrm{~s}$ \\
\hline 11- $\mathrm{CH}_{3}$ & $19.3, \mathrm{CH}_{3}$ & $1.08, \mathrm{~d}(7.0)$ \\
\hline 1 & $169.1, \mathrm{C}$ & \\
\hline 2 , & $126.7, \mathrm{C}$ & \\
\hline 3 , & 104.7, C & \\
\hline 4 , & $51.8, \mathrm{CH}$ & $3.48, \mathrm{p}(7.0)$ \\
\hline 5 , & $70.8, \mathrm{CH}$ & $3.69, \mathrm{p}(6.4)$ \\
\hline 6 , & $21.4, \mathrm{CH}_{3}$ & $1.06, \mathrm{~d}(6.4)$ \\
\hline 4'- $-\mathrm{CH}_{3}$ & $12.9, \mathrm{CH}_{3}$ & $1.04, \mathrm{~d}(7.0)$ \\
\hline
\end{tabular}




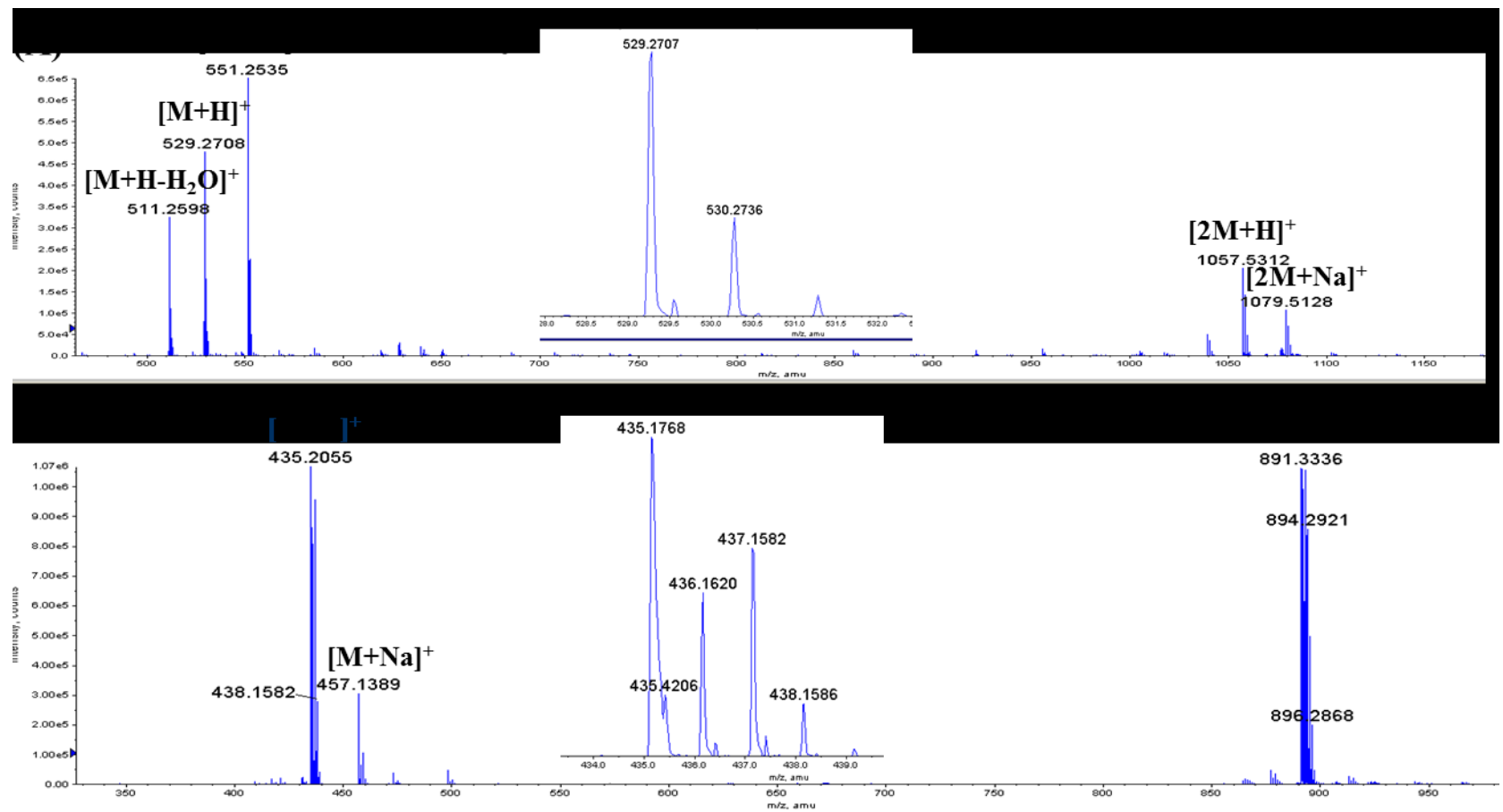

Figure 2.12: HRESIMS spectra of compounds 2.1 (A) and 2.4 (B) in positive mode. The $[\mathrm{M}+\mathrm{H}]^{+}$of each secondary metabolite is visualized to show the isotopic peak profile.

\section{Quantification of Major Secondary Metabolites}

The major metabolites quantified in the filtrate and mycelial extracts were chaetoglobosin A, chaetoglobosin F, chaetoglobosin C, chaetomugilin D, and chaetoviridin A (Table 2.4). Figure 2.13 shows a characteristic HPLC chromatogram from strain DAOM 240349 at 254 and $400 \mathrm{~nm}$ identifying each of the major metabolites. For quantification of metabolites, results are reported as the mean of three fermentations in Roux bottles normalized for mycelial mass.

The standard curves for each major metabolite were plotted as peak area counts versus the amount (mg) of compound on the column. The standard curves generated from the gradient elution program for chaetoglobosin $\mathrm{A}$, chaetoglobosin $\mathrm{F}$, chaetoglobosin $\mathrm{C}$, chaetomugilin $\mathrm{D}$, 
and chaetoviridin A each showed a linear relationship over the entire concentration range with $\mathrm{R}^{2}$ values of $0.99,1.00,0.99,0.99$, and 0.99 respectfully. The calibration curves of chaetoglobosin A and chaetomugilin D are displayed in figure 2.14. Using the standard curves, the recovery from spiked medium for chaetoglobosin A was $78.5 \pm 5.9 \%$, and, for chaetomugilin D $80.7 \pm$ 8.1\%. The limits of detection were: for chaetoglobosin A and F and chaetoviridin A, $10 \mathrm{ng}$; chaetoglobosin C, $100 \mathrm{ng}$; chaetomugilin D, $1 \mathrm{ng}$ and the corresponding limits of quantification were $15 \mathrm{ng}, 15 \mathrm{ng}, 20 \mathrm{ng}, 150 \mathrm{ng}$ and $3 \mathrm{ng}$. When all C. globosum strains were screened, the major metabolites observed in the filtrate extracts were chaetoglobosin A, chaetoglobosin F, chaetoglobosin $\mathrm{C}$, chaetomugilin $\mathrm{D}$, and chaetoviridin A (Table 2.4). Yields varied but the amounts of toxins other than chaetoviridin A were generally similar. Eight strains produced only trace amounts of chaetoviridin A. There was no evidence of chemotypes in the strains using cluster analysis with the Ward linkage method (SYSTAT v. 13). The same metabolites were retained in the mycelia at about $50 \%$ of the normalized values for liquid culture (Table 2.4). Chaetoviridin A was not observed in the mycelium and a number of strains only produced trace amounts. 
Table 2.4: Metabolite production by Canadian strains of C. globosum in the filtrate and mycelium extracts $\left(\mathrm{mg}^{-1} \mathrm{~g} \mathrm{dry} \mathrm{mycelium}^{-2}\right)^{\mathrm{a}}$.

\begin{tabular}{|c|c|c|c|c|c|c|c|c|c|}
\hline \multirow[t]{2}{*}{ Strain } & \multicolumn{5}{|c|}{ Filtrate } & \multicolumn{4}{|c|}{ Mycelium } \\
\hline & (2.1) & (2.2) & (2.3) & (2.4) & (2.5) & (2.1) & (2.2) & (2.3) & (2.4) \\
\hline 224120 & $7.84 \pm 1.77$ & $9.70 \pm 0.37$ & $3.24 \pm 1.02$ & $1.03 \pm 0.68$ & - & $0.54 \pm 0.18$ & $0.86 \pm 0.28$ & $1.61 \pm 0.52$ & $0.01 \pm 0.002$ \\
\hline 234120 & $16.50 \pm 2.90$ & $13.32 \pm 2.80$ & $6.11 \pm 1.58$ & $0.44 \pm 0.18$ & $0.12 \pm 0.06$ & $6.57 \pm 0.74$ & $1.90 \pm 0.25$ & $6.61 \pm 0.80$ & - \\
\hline 240348 & $11.66 \pm 2.60$ & $9.88 \pm 3.13$ & $11.54 \pm 4.78$ & $0.58 \pm 0.08$ & $0.04 \pm 0.02$ & $3.76 \pm 0.96$ & $1.66 \pm 0.44$ & $5.34 \pm 1.58$ & $0.05 \pm 0.02$ \\
\hline 240349 & $25.69 \pm 2.34$ & $6.39 \pm 0.49$ & $9.88 \pm 1.41$ & $2.69 \pm 0.36$ & $0.56 \pm 0.09$ & $4.07 \pm 1.03$ & $1.40 \pm 0.25$ & $3.22 \pm 0.54$ & - \\
\hline 240350 & $15.57 \pm 1.19$ & $4.21 \pm 1.36$ & $5.86 \pm 1.14$ & $1.05 \pm 0.17$ & $0.30 \pm 0.09$ & $6.38 \pm 0.47$ & $2.11 \pm 0.33$ & $12.48 \pm 0.91$ & - \\
\hline 240351 & $2.48 \pm 0.07$ & $6.28 \pm 1.56$ & $1.20 \pm 0.10$ & $1.64 \pm 0.21$ & $0.90 \pm 0.20$ & $0.10 \pm 0.005$ & $0.59 \pm 0.01$ & $0.72 \pm 0.02$ & $0.05 \pm 0.002$ \\
\hline 240352 & $8.12 \pm 2.73$ & $8.98 \pm 0.64$ & $6.63 \pm 3.38$ & $1.25 \pm 0.25$ & $1.55 \pm 1.20$ & $2.07 \pm 0.23$ & $0.83 \pm 0.11$ & $3.63 \pm 0.41$ & $0.33 \pm 0.05$ \\
\hline 240353 & $10.45 \pm 1.59$ & $9.97 \pm 0.44$ & $5.84 \pm 0.85$ & $1.09 \pm 0.44$ & $0.32 \pm 0.17$ & $7.85 \pm 1.03$ & $2.38 \pm 0.24$ & $5.43 \pm 0.34$ & - \\
\hline 240354 & $2.20 \pm 0.61$ & $3.97 \pm 0.55$ & $1.82 \pm 0.33$ & $1.40 \pm 0.03$ & $1.84 \pm 0.78$ & $0.35 \pm 0.03$ & $0.34 \pm 0.03$ & $0.87 \pm 0.07$ & $0.03 \pm 0.005$ \\
\hline 240355 & $4.47 \pm 0.36$ & $4.26 \pm 0.07$ & $6.13 \pm 0.20$ & $1.68 \pm 0.04$ & $1.19 \pm 0.12$ & $9.97 \pm 0.29$ & $1.71 \pm 0.19$ & $16.43 \pm 0.94$ & - \\
\hline 240356 & $11.33 \pm 2.21$ & $8.06 \pm 1.36$ & $8.87 \pm 2.66$ & $0.92 \pm 0.64$ & $1.14 \pm 1.10$ & $4.30 \pm 0.50$ & $2.10 \pm 0.20$ & $9.43 \pm 0.80$ & $0.13 \pm 0.006$ \\
\hline 240357 & $1.67 \pm 0.27$ & $3.13 \pm 1.04$ & $4.41 \pm 1.26$ & $1.91 \pm 0.04$ & - & $1.88 \pm 0.21$ & $1.72 \pm 0.15$ & $4.73 \pm 0.42$ & - \\
\hline 240358 & $2.28 \pm 1.05$ & $2.79 \pm 0.14$ & $0.96 \pm 0.22$ & $0.12 \pm 0.04$ & - & $1.81 \pm 063$ & $0.39 \pm 0.06$ & $0.84 \pm 0.45$ & - \\
\hline 240359 & $3.44 \pm 1.06$ & $1.10 \pm 0.48$ & $1.41 \pm 0.30$ & $1.32 \pm 0.07$ & $2.02 \pm 0.65$ & $2.40 \pm 1.42$ & $0.30 \pm 0.13$ & $1.01 \pm 0.51$ & $0.36 \pm 0.13$ \\
\hline 242036 & $7.59 \pm 1.25$ & $12.81 \pm 1.09$ & $7.99 \pm 2.18$ & $0.56 \pm 0.13$ & $0.39 \pm 0.15$ & $5.74 \pm 0.21$ & $4.06 \pm 0.37$ & $8.37 \pm 0.37$ & - \\
\hline 242037 & $9.80 \pm 1.13$ & $7.71 \pm 0.60$ & $5.50 \pm 0.39$ & $0.50 \pm 0.34$ & - & $7.45 \pm 1.64$ & $1.38 \pm 0.30$ & $5.14 \pm 1.49$ & $0.03 \pm 0.02$ \\
\hline 242038 & $7.98 \pm 0.79$ & $12.58 \pm 2.56$ & $8.63 \pm 0.77$ & $1.61 \pm 0.43$ & $1.19 \pm 0.59$ & $2.44 \pm 0.10$ & $2.10 \pm 0.09$ & $5.45 \pm 0.29$ & - \\
\hline 242039 & $4.24 \pm 1.30$ & $10.65 \pm 0.82$ & $4.06 \pm 1.92$ & $1.55 \pm 0.82$ & $5.82 \pm 2.09$ & $0.60 \pm 0.11$ & $1.45 \pm 0.23$ & $3.21 \pm 0.32$ & $0.37 \pm 0.02$ \\
\hline 242040 & $5.41 \pm 1.05$ & $5.66 \pm 0.35$ & $2.13 \pm 0.14$ & $1.14 \pm 0.11$ & $0.52 \pm 0.35$ & $1.29 \pm 0.14$ & $0.36 \pm 0.06$ & $0.90 \pm 0.09$ & $0.04 \pm 0.006$ \\
\hline 242041 & $1.04 \pm 0.04$ & $4.25 \pm 1.02$ & $7.99 \pm 0.66$ & $2.93 \pm 0.19$ & - & $0.98 \pm 0.10$ & $1.06 \pm 0.06$ & $3.02 \pm 0.24$ & $0.42 \pm 0.02$ \\
\hline 242042 & $4.09 \pm 0.83$ & $2.56 \pm 0.16$ & $1.98 \pm 0.25$ & $0.99 \pm 0.48$ & $1.06 \pm 0.28$ & $0.70 \pm 0.26$ & $0.17 \pm 0.08$ & $0.51 \pm 0.02$ & $0.08 \pm 0.03$ \\
\hline 242043 & $9.93 \pm 3.10$ & $12.08 \pm 3.53$ & $8.36 \pm 3.36$ & $1.19 \pm 0.83$ & $0.93 \pm 0.62$ & $2.24 \pm 0.37$ & $2.40 \pm 0.43$ & $7.32 \pm 1.23$ & $0.47 \pm 0.09$ \\
\hline 242044 & $11.99 \pm 2.04$ & $4.26 \pm 0.81$ & $5.41 \pm 1.47$ & $0.14 \pm 0.06$ & - & $13.08 \pm 1.07$ & $1.76 \pm 0.27$ & $11.70 \pm 1.81$ & - \\
\hline $7142^{b}$ & $8.39 \pm 2.75$ & $10.49 \pm 2.98$ & $5.32 \pm 1.75$ & $1.11 \pm 0.54$ & $0.26 \pm 0.15$ & $3.06 \pm 0.31$ & $0.72 \pm 0.12$ & $1.85 \pm 0.29$ & $0.04 \pm 0.01$ \\
\hline $7773^{b}$ & $4.05 \pm 0.65$ & $6.15 \pm 0.60$ & $2.39 \pm 0.29$ & $0.84 \pm 0.32$ & $0.14 \pm 0.05$ & $0.10 \pm 0.04$ & $0.72 \pm 0.26$ & $0.99 \pm 0.35$ & $0.01 \pm 0.002$ \\
\hline Mean & $7.93 \pm 1.43$ & $7.24 \pm 1.16$ & $5.35 \pm 1.29$ & $1.18 \pm 0.30$ & $1.07 \pm 0.46$ & $3.59 \pm 0.48$ & $1.38 \pm 0.20$ & $4.83 \pm 0.59$ & $0.16 \pm 0.04$ \\
\hline
\end{tabular}

a. mean of three replicate fermentations

b. UAMH, all other strains deposited with DAOM

- Below limit of detection. 


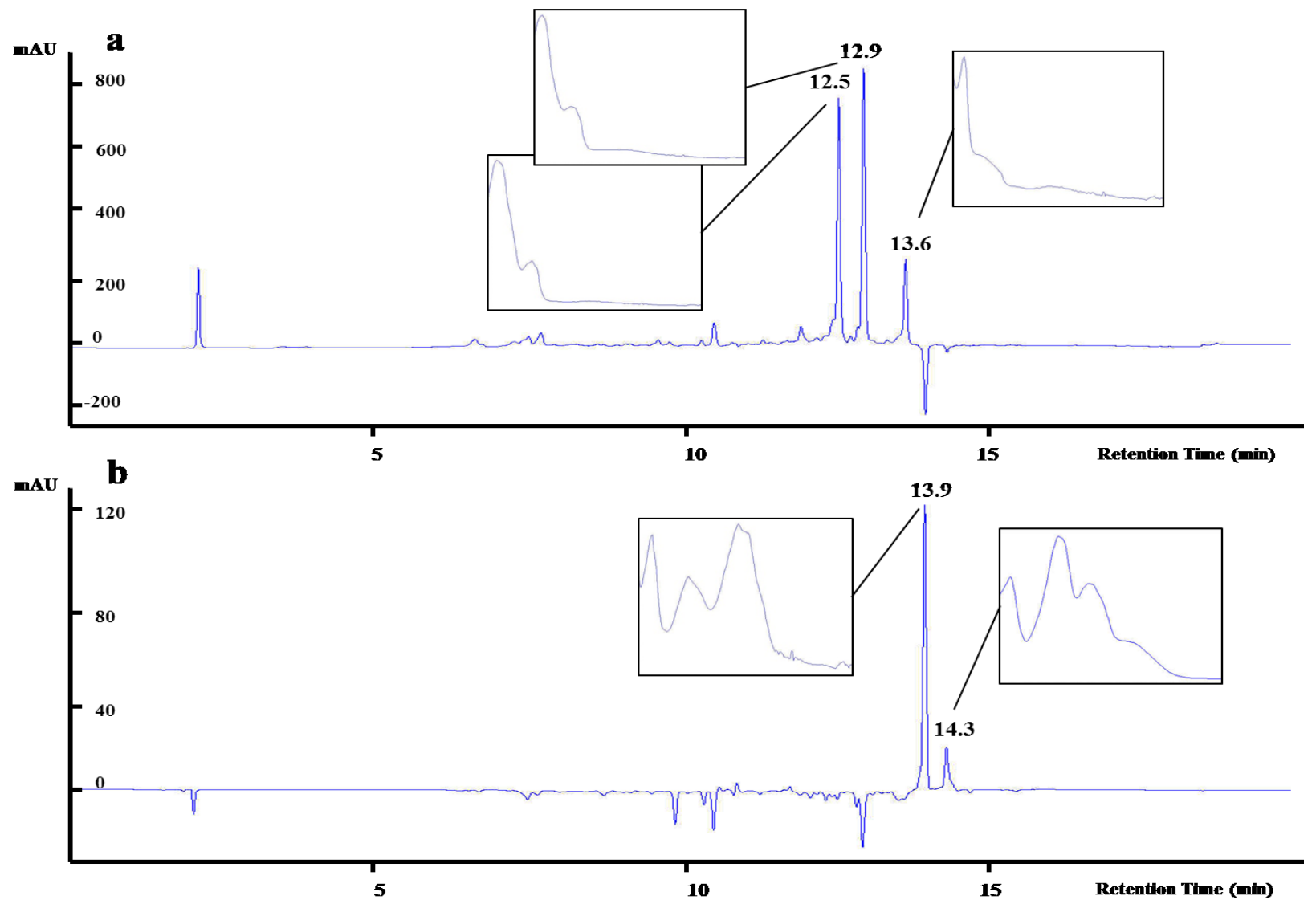

Figure 2.13: HPLC chromatogram of C. globosum DAOM 240349 filtrate extract at $254 \mathrm{~nm}$ (a) and $400 \mathrm{~nm}$ (b) with associated UV spectra (200-600nm) of major metabolites.
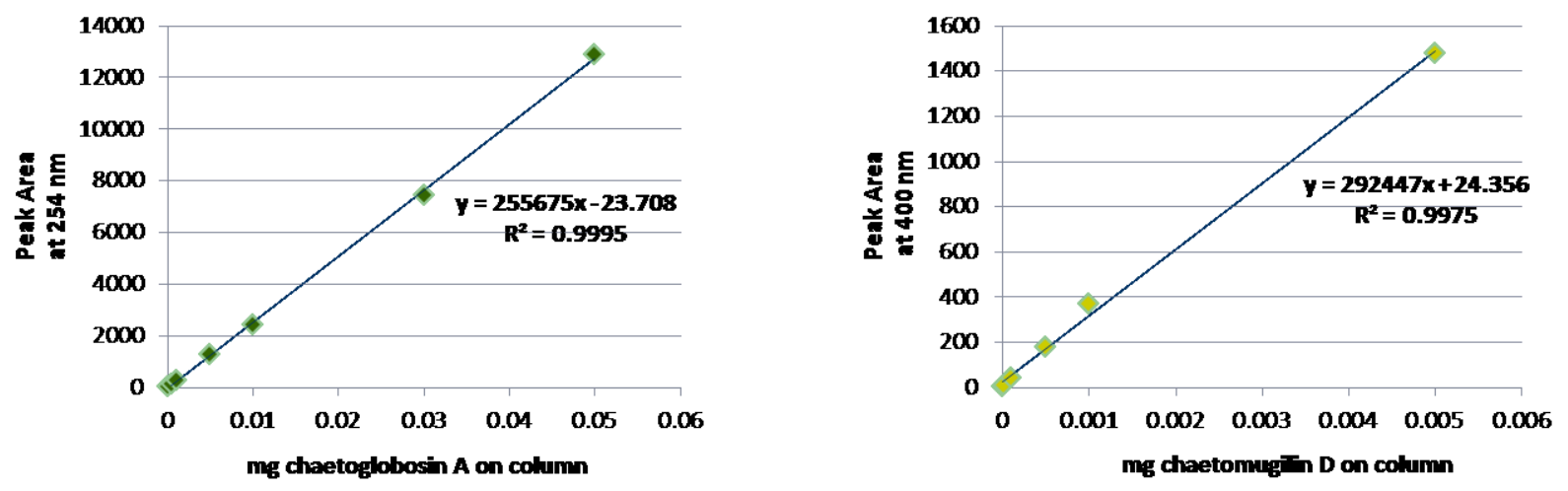

Figure 2.14: Calibration plots for chaetoglobosin A (2.1) and chaetomugilin D (2.4). 


\section{Structural Characterization of New Azaphilones from C. globosum DAOM 240359}

Compound 2.6 was isolated as an optically active dark red gum with the molecular formula $\mathrm{C}_{25} \mathrm{H}_{30} \mathrm{NO}_{6} \mathrm{Cl}$ determined by the HRESIMS in positive mode at $m / z 476.1847[\mathrm{M}+\mathrm{H}]^{+}$. A 3:1 isotopic peak ratio for $[\mathrm{M}+\mathrm{H}]^{+}:[\mathrm{M}+\mathrm{H}+2]^{+}$was observed indicating the presence of a single

chlorine in the molecule. The odd molecular weight in addition to the interpretation of the ${ }^{13} \mathrm{C}$ and ${ }^{1} \mathrm{H}$ NMR spectra suggest the presence of a single nitrogen atom and eleven units of unsaturation attributed to three rings and eight double bonds.

The ${ }^{1} \mathrm{H}$ spectrum indicated the presence of four aliphatic methyl groups at $\delta 0.91(\mathrm{t}, J=7.5), \delta$ $1.03(\mathrm{~d}, 7.0), \delta 1.09(\mathrm{~d}, J=6.7), \delta 1.11(\mathrm{~d}, J=6.4)$, a downfield tertiary methyl at $\delta 1.67$, a multiplet methylene at $\delta 1.47$ and a multiplet methine at $\delta 2.33$. Two methines were observed at $\delta 3.51(\mathrm{p}, J=6.4 / 7.0)$ and $\delta 3.95(\mathrm{p}, J=6.4 / 7.0)$ suggesting they were vicinal. Two additional substituted methylenes that were not observed in the ${ }^{1} \mathrm{H}$ spectrum of chaetoviridin $\mathrm{A}(\mathbf{2 . 5})$ appeared at $\delta 3.75(\mathrm{t}, J=5.1)$ and $\delta 4.08(\mathrm{~m})$. Two proton signals at $\delta 6.44(\mathrm{~d}, J=15.4)$ and $\delta 6.49$ $(\mathrm{dd}, J=6.2,15.4)$ illustrate a trans-olefinic group due their chemical shifts and large coupling constants. Two unsaturated proton singlets at $\delta 6.99$ and $\delta 7.92$ are similar to those of chaetoviridin A (2.5) but chemical shift differences suggested the nitrogen at position 2 instead of an oxygen.

The ${ }^{13} \mathrm{C}$ spectrum displayed twenty five carbon signals of which ten were quaternary which is indicative of a highly conjugated, planar structure. These signals were a result of a chlorine bearing carbon at $\delta 95.3$, three carbonyls at $\delta 171.0, \delta 193.4$, and $\delta 201.1$, an oxygenated carbon at $\delta 87.7$, and five conjugated $\mathrm{sp}^{2}$ carbons at $\delta 109.0, \delta 116.6, \delta 145.7, \delta 151.2$, and $\delta 165.5$. 
These data are similar to that of chaetoviridin A (2.5) reported by Takahashi et al. (1990) but again chemical shift differences suggests that the lone nitrogen atom occurs at position 2 .

Analysis of the COSY, HSQC, and HMBC identified the 3-methyl-1-pentenyl and 2-butanol-3yl moieties as in chaetoviridin A (2.5). The HMBC correlation between H-9 and C-3 attaches the 3-methyl-1-pentyl chain to C-3. HMBC correlations to C-3' from H-4', H-4'-Me, and H-5' attaches the 2-butanol-3-yl substituent to the conjugated carbonyl. The COSY spectrum showed that the two substituted methylenes $(\delta 3.75$ and $\delta 4.08)$ not present in chaetoviridin $\mathrm{A}(\mathbf{2 . 5})$ are vicinal and an $\mathrm{HMBC}$ correlation from $\mathrm{H}-1$ to $\mathrm{C}-1$ " indicates the nitrogen occupies position 2 and bears a hydroxy ethyl group analogous to isochromophilone VI (Arai et al. 1995). The ${ }^{1} \mathrm{H}$ and ${ }^{13} \mathrm{C}$ assignments of $\mathbf{2 . 6}$ are reported in table 2.5 and observed COSY and HMBC correlations are illustrated in figure 2.16 .

The structure of $\mathbf{2 . 6}$ was established as a $N$-2- hydroxy ethyl derivative of chaetoviridin A. The optical rotation of 2.6, $[\alpha]_{\mathrm{D}}-40(\mathrm{c} 0.01, \mathrm{MeOH})$, has the opposite sign compared to chaetoviridin

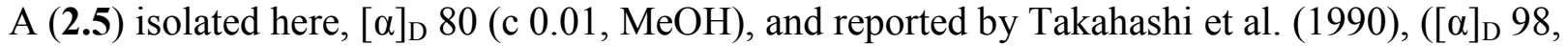
c 0.05), suggesting a change in stereochemistry. The stereochemistry of the C-7 and C-11 methyl moieties were both determined to be of the $(S)$ configuration by Takahashi et al. (1990) and later confirmed by X-ray crystallography (Borges et al. 2011).

Comparison of our ${ }^{1} \mathrm{H},{ }^{13} \mathrm{C}$ (Table 2.5) and NOE data for compounds 2.5 and $\mathbf{2 . 6}$ demonstrate the same $(S)$ configuration at these two stereocenters. A strong NOE correlation between $\mathrm{H}-9 / \mathrm{H}-11$ and a weak correlation between $\mathrm{H}-9 / \mathrm{H}-11-\mathrm{Me}$ in both compounds $\mathbf{2 . 5}$ and $\mathbf{2 . 6}$ also confirms the 
stereochemistry at C-11 as $(S)$, consistent with reported chaetovirdins (Takahashi et al. 1990), chaetomugilins (Muroga et al. 2009; Yasuhide et al. 2008) and isochromophilones (Arai et al. 1995). Germain et al. (2011) who examined stereoselective synthesis of sclerotiorin compounds at the C-7 position showed that a nitrogen bearing a non-chiral moiety does not affect the sign of the optical rotation. Together, this data suggests that the difference in stereochemistry accounting

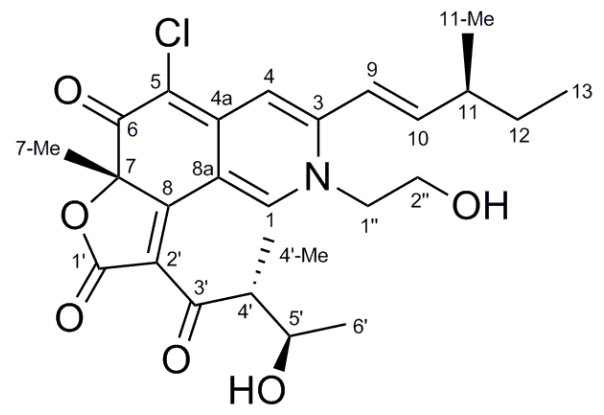

Figure 2.15: Structure of 4'-epi- $N$-2-hydroxyethyl-azachaetoviridin A (2.6).

Table 2.5: ${ }^{1} \mathrm{H}(400 \mathrm{MHz})$ and ${ }^{13} \mathrm{C}$ NMR $(100 \mathrm{MHz})$ data for 2.6 in $\mathrm{CD}_{3} \mathrm{CN}$.

\begin{tabular}{|c|c|c|}
\hline \multirow[b]{2}{*}{ Position } & \multicolumn{2}{|c|}{$(2.6)$} \\
\hline & $\delta_{\mathrm{C}}$, type & $\delta_{\mathrm{H}}(J, \mathbf{H z})$ \\
\hline 1 & $143.2, \mathrm{CH}$ & $7.92, \mathrm{~s}$ \\
\hline 3 & $151.2, \mathrm{C}$ & \\
\hline 4 & $113.0, \mathrm{CH}$ & $6.99, \mathrm{~s}$ \\
\hline $4 a$ & $145.7, \mathrm{C}$ & \\
\hline 5 & $95.3, \mathrm{C}$ & \\
\hline 6 & $193.4, \mathrm{C}$ & \\
\hline 7 & $87.7, \mathrm{C}$ & \\
\hline 8 & $165.5, \mathrm{C}$ & \\
\hline $8 \mathbf{8}$ & $116.6, \mathrm{C}$ & \\
\hline 9 & $121.1, \mathrm{CH}$ & $6.44, \mathrm{~d}(15.4)$ \\
\hline 10 & $150.5, \mathrm{CH}$ & $6.49, \mathrm{dd}(6.2,15.5)$ \\
\hline 11 & $40.0, \mathrm{CH}$ & $2.33, \mathrm{~m}$ \\
\hline 12 & $29.6, \mathrm{CH}_{2}$ & $1.47, \mathrm{~m}$ \\
\hline 13 & $12.0, \mathrm{CH}_{3}$ & $0.91, \mathrm{t}(7.5)$ \\
\hline 7- $\mathrm{CH}_{3}$ & $30.0, \mathrm{CH}_{3}$ & $1.67, \mathrm{~s}$ \\
\hline $11-\mathrm{CH}_{3}$ & $19.5, \mathrm{CH}_{3}$ & $1.09, \mathrm{~d}(6.7)$ \\
\hline 1 ' & 171.0, C & \\
\hline 2' & $109.0, \mathrm{C}$ & \\
\hline 3, & 201.1, C & \\
\hline 4 ' & $52.5, \mathrm{CH}$ & $3.51, \mathrm{p}(6.4,7.0)$ \\
\hline 5 , & $69.7, \mathrm{CH}$ & $3.69, p(6.4,7.0)$ \\
\hline $6^{\prime}$ & $20.6, \mathrm{CH}_{3}$ & $1.11, \mathrm{~d}(6.4)$ \\
\hline 4'- $\mathrm{CH}_{3}$ & $12.5, \mathrm{CH}_{3}$ & $1.02, \mathrm{~d}(7.0)$ \\
\hline $1 "$ & $57.2, \mathrm{CH}_{2}$ & $4.08, \mathrm{~m}$ \\
\hline 2" & $60.5, \mathrm{CH}_{2}$ & $3.75, \mathrm{t}(5.1)$ \\
\hline
\end{tabular}


for the opposite sign of the optical rotation between $\mathbf{2 . 5}$ and $\mathbf{2 . 6}$ is attributed to the 2-butanol-3-yl moiety. A difference was observed between the H-5' chemical shifts of $2.5(\delta 3.69)$ and $\mathbf{2 . 6}(\delta$ 3.95). Comparison of chemical shifts for positions C-4', C-5', and C-6' of compound $\mathbf{2} .6$ to that of chaetoviridin A, 4'-epi-chaetoviridin A, and 5'-epi-chaetoviridin A as reported by Borges et al. (2011) suggests the $(R)$ configuration for position C-5'. NOE correlations were observed between $\mathrm{H}-4^{\prime} / \mathrm{H}-6^{\prime}$ and $\mathrm{H}-4^{\prime}-\mathrm{Me} / \mathrm{H}-5^{\prime}$ ' but no NOE was observed between $\mathrm{H}-4^{\prime}$ ' and $\mathrm{H}-5^{\prime}$ ' that is consistent with data for 4'-epi-chaetoviridin A indicating the stereochemistry at C-4' and C5' are $(R)$ and $(R)$ respectively for 2.6. Furthermore, invoking a hydrogen bond stabilization between the C-3' carbonyl and 5' hydroxyl confirms the observed NOE's and the coupling constant between H-4' and H-5' of 7.0 Hz. A similar argument was utilized by Takahashi et al. (1990) to establish the stereochemistry of C-4' and C-5' as $(S)$ and $(R)$ respectively of chaetoviridin A. The single point stereochemical difference at position C-4' accounts for the opposite sign of the optical rotation compared to chaetoviridin A. Thus, the structure of $\mathbf{2 . 6}$ was established as 4'-epi$N$-2-hydroxyethyl-azachaetoviridin A, figure 2.15.
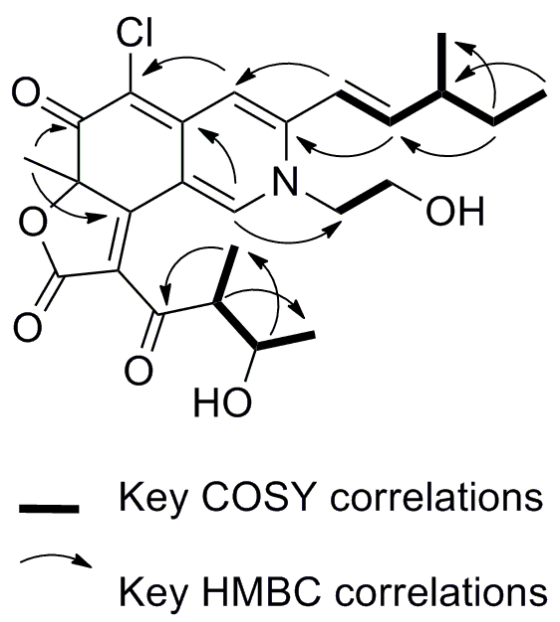

Figure 2.16: Observed COSY and key HMBC correlations for compound 2.6. 
4'-epi- $N$-2-hydroxyethyl-azachaetoviridin A (2.6); $4.1 \mathrm{mg}$; optically active red gum; $[\alpha]_{\mathrm{D}}-40$ (c 0.01, MeOH); UV (MeOH)/nm $\lambda_{\max }(\log \varepsilon) 267$ (4.22), 435 (3.98), 515 (4.01); HRESIMS $m / z$ $476.1847[\mathrm{M}+\mathrm{H}]^{+}$(calculated for $\mathrm{C}_{25} \mathrm{H}_{31} \mathrm{NO}_{6} \mathrm{Cl}, 476.1840$ ). ${ }^{1}$ Hand ${ }^{13} \mathrm{C}$ NMR data and are reported in table 2.5. The structure of $\mathbf{2 . 6}$ can be found in figure 2.15 .

Compound 2.7 was isolated as an optically active orange gum with the molecular formula $\mathrm{C}_{27} \mathrm{H}_{30} \mathrm{NO}_{6} \mathrm{Cl}$ determined from the HRESI-MS in positive mode at $m / z 500.1867[\mathrm{M}+\mathrm{H}]^{+}$. A similar 3:1 isotopic peak ratio was observed and the odd molecular weight suggests a single chlorine and nitrogen in the molecule. The thirteen units of unsaturation were attributed to ten double bonds and three rings indicating two additional double bonds compared to compound $\mathbf{2 . 6}$ plus the addition of two carbon atoms.

The ${ }^{1} \mathrm{H}$ spectrum displayed many of the features of compound 2.7 including two aliphatic methyl groups at $\delta 0.95(\mathrm{t}, J=7.5), \delta 1.14(\mathrm{~d}, J=7.0)$, a tertiary methyl at $\delta 1.65$, a multiplet methylene at $\delta 1.50$, and a multiplet methine at $\delta 2.38$. The proton singlets at $\delta 7.02, \delta 7.89$ as well as the two proton signals at $\delta 6.51(\mathrm{dd}, J=7.6,15.5)$ and $\delta 6.64(\mathrm{~d}, J=15.5)$ that are similar to compounds $\mathbf{2 . 6}$ and chaetoviridin A (2.5) indicate the same chlorinated, conjugated lactone structure with a 9,10 trans double bond. In contrast to $\mathbf{2 . 6}$, two downfield methyl groups at $\delta 1.86$ and $\delta 1.87$ (d, $J=6.7)$ as well as a quartet methine at $\delta 6.60(\mathrm{q}, J=6.8)$ identify a 2-butene-3-yl moiety as observed in chaetoviridin E (Phonkerd et al. 2008). Finally, three vicinal methylene groups were observed at $\delta 4.12, \delta 2.00$, and $\delta 2.36$ suggesting a structurally different chain from the $N-2$ position compared to the hydroxyethyl moiety observed in compound $\mathbf{2 . 6}$. 
The ${ }^{13} \mathrm{C}$ spectrum of $\mathbf{2 . 7}$ was similar to that of $\mathbf{2 . 6}$ (refer to Table 2.6 and 2.5 respectively) however two additional resonances were observed including a carboxylic acid functionality at $\delta$ 176.3 and methylene at $\delta 31.4$. A new carbon double bond was observed at $\delta 139.1$ and $\delta 147.5$, concomitant with the absence of the C-4' and C-5' methines of 2.6. The C-2" of 2.6 at $\delta 60.5$ has been replaced by a methylene at $\delta 26.6$ indicating the loss of the hydroxy group at this position.

Analysis of the COSY, HSQC and HMBC revealed the same core structure as $\mathbf{2 . 6}$ but with differences in the N-2 and C-3' conjugated carbonyl side chains. COSY correlations from H-2" to H-1" and H-3" as well as HMBC correlations from H-2" and H-3" to the carboxylic acid C-4" ( $\delta$ 176.3) establishes the $\mathrm{N}-2$ side chain as gamma butyric acid. HMBC correlations between C4'-Me singlet $(\delta 1.86)$ to ${ }^{13} \mathrm{C}$ resonances at $\delta 192.1, \delta 139.1$, and $\delta 147.5$ as well as between the methyl doublet at $\delta 1.87$ to $\delta 139.1$ and $\delta 147.5$ establishes a 2-butene-3-yl function at C-3'. This represents a beta oxidation at the C-4', C-5' over $\mathbf{2 . 6}$ as seen in chaetoviridin E (Phonkerd et al. 2008).

NOE correlations between $\mathrm{H}-9 / \mathrm{H}-11, \mathrm{H}-9 / \mathrm{H}-11-\mathrm{Me}$ and $\mathrm{H}-10 / \mathrm{H}-12$ establish the stereochemistry at the C-11 position as $(S)$ as in $\mathbf{2 . 5}$ and 2.6. Absence of an NOE between H-4'-Me and H-5' as well as similar chemical shifts indicates the configuration of the double bond as $(E)$ as in chaetoviridin E. The 4' and 6' methyl moieties are too close in chemical shift to observe the confirming NOE. Additional NOE correlations between $\mathrm{H}-1 / \mathrm{H}-1$ ” and $\mathrm{H}-2$ "/H-9 indicate interactions between the planar pyrano quinone core and the flexible side chain off the nitrogen, which was also observed in 2.6. The optical rotation of 2.7, $[\alpha]_{\mathrm{D}}-80(\mathrm{c} 0.01, \mathrm{MeOH})$, is of the 
same sign as chaetoviridin $\mathrm{E},[\alpha]_{\mathrm{D}}-385\left(\mathrm{c} 0.02, \mathrm{CHCl}_{3}\right)$ (Phonkerd et al. 2008), establishing the structure of $\mathbf{2 . 7}$ as $N$-2-butyric-azochaetoviridin E.

$N$-2-butyric-azochaetoviridin E (2.7); $6.1 \mathrm{mg}$; optically active orange gum; $[\alpha]_{\mathrm{D}}-80$ (c 0.01 , $\mathrm{MeOH}) ; \mathrm{UV}(\mathrm{MeOH}) / \mathrm{nm} \lambda_{\max }(\log \varepsilon) 238$ (2.66), 294 (2.48), 348 (2.54), 390 (1.77), 472 (0.41); HRESIMS $m / z 500.1867[\mathrm{M}+\mathrm{H}]]^{+}$(calculated for $\mathrm{C}_{27} \mathrm{H}_{31} \mathrm{NO}_{6} \mathrm{Cl}$ : 500.1840). ${ }^{1} \mathrm{Hand}{ }^{13} \mathrm{C}$ NMR data and are reported in table 2.6. The structure of $\mathbf{2 . 7}$ can be found in figure 2.17.

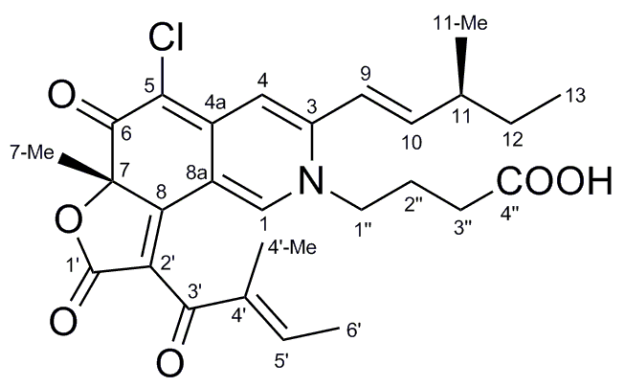

Figure 2.17: Structure of $N$-2-butyric-azochaetoviridin E (2.7).

Compound $\mathbf{2 . 8}$ was isolated as an optically active orange gum with the molecular formula $\mathrm{C}_{18} \mathrm{H}_{22} \mathrm{NO}_{4} \mathrm{Cl}$ determined by HRESI-MS at $m / z 352.1379[\mathrm{M}+\mathrm{H}]^{+}$. Again, the 3:1 isotopic ratio of $[\mathrm{M}+\mathrm{H}]^{+}:[\mathrm{M}+\mathrm{H}+2]^{+}$was observed in the mass spectrum and the odd molecular weight suggested the presence a single chlorine and nitrogen in the molecule. The eight units of unsaturation calculated from the molecular formula were a result of six double bonds and two rings indicating a planar, isochromophilone structure (Arai et al. 1995).

The ${ }^{1} \mathrm{H}$ spectrum of $\mathbf{2 . 8}$ showed characteristic chemical shifts of the pyrano quinone structure as in 2.4-2.7. Two vicinal substituted methylene signals at $\delta 3.76(\mathrm{t}, J=5.2)$ and $\delta 4.06(\mathrm{t}, J=5.2)$ were observed as in $\mathbf{2 . 6}$ identifying the same $\mathrm{N}$-2-hydroxy ethyl side chain. The ${ }^{13} \mathrm{C}$ spectrum 
Table 2.6: ${ }^{1} \mathrm{H}(400 \mathrm{MHz})$ and ${ }^{13} \mathrm{C}$ NMR $(100 \mathrm{MHz})$ data for 2.7 in $\mathrm{CD}_{3} \mathrm{OD}$.

\begin{tabular}{|c|c|c|}
\hline \multirow[b]{2}{*}{ Position } & \multicolumn{2}{|c|}{$(2.7)$} \\
\hline & $\delta_{\mathrm{C}, \text { type }}$ & $\delta_{\mathrm{H}}(J, \mathbf{H z})$ \\
\hline 1 & $140.3, \mathrm{CH}$ & $7.89, \mathrm{~s}$ \\
\hline 3 & 151.1, C & \\
\hline 4 & $111.9, \mathrm{CH}$ & $7.02, \mathrm{~s}$ \\
\hline $4 a$ & $148.2, \mathrm{C}$ & \\
\hline 5 & $99.4, \mathrm{C}$ & \\
\hline 6 & $182.0, \mathrm{C}$ & \\
\hline 7 & $89.9, \mathrm{C}$ & \\
\hline 8 & $164.3, \mathrm{C}$ & \\
\hline $8 a$ & $113.8, \mathrm{C}$ & \\
\hline 9 & $121.2, \mathrm{CH}$ & $6.64, \mathrm{~d}(15.3)$ \\
\hline 10 & $151.2, \mathrm{CH}$ & $6.51, \mathrm{dd}(7.6,15.5)$ \\
\hline 11 & $40.6, \mathrm{CH}$ & $2.38, \mathrm{~m}$ \\
\hline 12 & $30.2, \mathrm{CH}_{2}$ & $1.50, \mathrm{~m}$ \\
\hline 13 & $12.2, \mathrm{CH}_{3}$ & $0.95, \mathrm{t}(7.5)$ \\
\hline 7- $\mathrm{CH}_{3}$ & $26.4, \mathrm{CH}_{3}$ & $1.65, \mathrm{~s}$ \\
\hline 11- $\mathrm{CH}_{3}$ & $19.7, \mathrm{CH}_{3}$ & $1.14, \mathrm{~d}(7.0)$ \\
\hline 1 & $170.1, \mathrm{C}$ & \\
\hline 2 , & 125.7, C & \\
\hline 3 , & 192.1, C & \\
\hline 4 ' & $139.1 \mathrm{C}$ & \\
\hline 5 , & $147.5, \mathrm{CH}$ & $6.60, q(6.8)$ \\
\hline $6^{\prime}$ & $15.3, \mathrm{CH}_{3}$ & $1.87, \mathrm{~d}(6.7)$ \\
\hline $4^{\prime}-\mathrm{CH}_{3}$ & $10.7, \mathrm{CH}_{3}$ & $1.86, \mathrm{~s}$ \\
\hline $1 \%$ & $55.1, \mathrm{CH}_{2}$ & $4.12, \mathrm{t}(7.6)$ \\
\hline 2 ', & $26.6, \mathrm{CH}_{2}$ & $2.00, \mathrm{~m}$ \\
\hline 3"' & $31.4, \mathrm{CH}_{2}$ & $2.36, \mathrm{t}(5.6)$ \\
\hline 4 "' & $176.3, \mathrm{C}$ & \\
\hline
\end{tabular}

also showed pyrano-quinone characteristic chemical shifts as in 2.4-2.7. The same carbon chemical shifts for the N-2-hydroxy ethyl side chain were also observed as in $\mathbf{2 . 6}$ (Table 2.5 and Table 2.7). Interpretation of the COSY, HSQC and HMBC spectra of 2.8 identified the same 3methyl-1-pentyl moiety present in other characterized azaphilones. No other aliphatic moieties were observed and the C-8 chemical shift of $\delta 197.8$ indicates a ketone as opposed to the fused lactone ring observed in compounds 2.4-2.7 isolated from the same EtOAc filtrate extract.

The stereochemistry at C-7 and C-11 were determined to be of the $(S)$ configuration when comparing the optical rotation, $[\alpha]_{\mathrm{D}}-90(\mathrm{c} 0.2, \mathrm{MeOH})$, to that of compounds isolated here and to 
the literature (Arai et al. 1995). The negative value of the optical rotation is also consistent with Germain et al. (2011) who examined stereoselective synthesis of sclerotiorin compounds at the C-7 position. Similar natural products where both C-11 and C-7 are $(S)$ had a negative optical rotation; however, when $\mathrm{C}-7$ was $(R)$ the sign switched to positive with the same magnitude. This demonstrated that a nitrogen bearing, non-chiral moiety does not affect the sign of the optical rotation. The structure of $\mathbf{2 . 8}$ was determined to be (E)-5-chloro-7-hydroxy-2-(2hydroxyethyl)-7-methyl-3-(3-methylpent-1-en-1-yl)-isoquinoline-6, 8-(2H,7H)-dione but given the name isochromophilone XIII based on the pyrano quinone presence and the lack of fused lactone ring.

Isochromophilone XIII (2.8); $9.8 \mathrm{mg}$; optically active orange gum; $[\alpha]_{\mathrm{D}}-90$ (c $\left.0.2, \mathrm{MeOH}\right)$; UV $(\mathrm{MeOH}) / \mathrm{nm} \lambda_{\max }(\log \varepsilon) 204$ (2.66), 277 (2.48), 379 (2.54), 463 (1.77); HRESIMS m/z 352.1379 $[\mathrm{M}+\mathrm{H}]^{+}$(calculated for $\left.\mathrm{C}_{18} \mathrm{H}_{23} \mathrm{NO}_{4} \mathrm{Cl}: 352.1316\right)$. ${ }^{1} \mathrm{Hand}{ }^{13} \mathrm{C} \mathrm{NMR}$ data and are reported in table 2.7. The structure of $\mathbf{2 . 8}$ can be found in figure 2.18 .

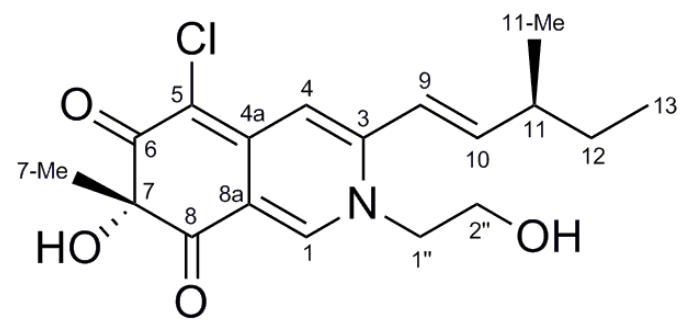

Figure 2.18: Structure of Isochromophilone XIII (2.8). 
Table 2.7: ${ }^{1} \mathrm{H}(400 \mathrm{MHz})$ and ${ }^{13} \mathrm{C}$ NMR $(100 \mathrm{MHz})$ data for 2.8 in $\mathrm{CD}_{3} \mathrm{CN}$.

\begin{tabular}{|c|c|c|}
\hline \multirow[b]{2}{*}{ Position } & \multicolumn{2}{|c|}{$(2.8)$} \\
\hline & $\delta_{\mathrm{C}}$, type & $\delta_{\mathrm{H}}(J, \mathbf{H z})$ \\
\hline 1 & $142.7, \mathrm{CH}$ & $7.87, \mathrm{~s}$ \\
\hline 3 & $150.7, \mathrm{C}$ & \\
\hline 4 & $111.6, \mathrm{CH}$ & $6.94, \mathrm{~s}$ \\
\hline $4 a$ & $147.7, \mathrm{C}$ & \\
\hline 5 & $98.8, \mathrm{C}$ & \\
\hline 6 & 187.7, C & \\
\hline 7 & $84.2, \mathrm{C}$ & \\
\hline 8 & $197.8, \mathrm{C}$ & \\
\hline $8 \mathbf{a}$ & $116.2, \mathrm{C}$ & \\
\hline 9 & $121.0, \mathrm{CH}$ & $6.43, \mathrm{~d}(15.5)$ \\
\hline 10 & $149.9, \mathrm{CH}$ & 6.44, dd $(6.1,15.5)$ \\
\hline 11 & $40.0, \mathrm{CH}$ & $2.32, \mathrm{~m}$ \\
\hline 12 & $29.8, \mathrm{CH}_{2}$ & $1.46, \mathrm{~m}$ \\
\hline 13 & $12.0, \mathrm{CH}_{3}$ & $0.91, \mathrm{t}(7.4)$ \\
\hline $7-\mathrm{CH}_{3}$ & $29.4, \mathrm{CH}_{3}$ & $1.43, \mathrm{~s}$ \\
\hline 11- $\mathrm{CH}_{3}$ & $19.5, \mathrm{CH}_{3}$ & $1.10, \mathrm{~d}(6.7)$ \\
\hline 1 , & $56.8, \mathrm{CH}_{2}$ & $4.06, \mathrm{t}(5.2)$ \\
\hline 2', & $60.7, \mathrm{CH}_{2}$ & $3.76, \mathrm{t}(5.2)$ \\
\hline
\end{tabular}

\section{Antimicrobial activity of purified $C$. globosum metabolites}

In vitro, $200 \mu \mathrm{M}$ of compounds 2.1, 2.2, 2.4, 2.7 and 2.8, respectively, resulted in significant reduction in the growth of the Gram positive bacterium Bacillus subtilis and the Gram negative bacterium Psuedomonas putida (ANOVA, Tukey's Test, $\mathrm{P}<0.05$ ) (refer to Table 2.8).

Additionally, $20 \mu \mathrm{M}$ concentrations of compounds 2.1, 2.2, 2.4 and 2.7 reduced the growth of both species. $200 \mu \mathrm{M}$ of $\mathbf{2 . 4}$ and $\mathbf{2 . 7}$, respectively, gave similar results compared to the same concentration of the positive control, chloramphenicol; whereas, the rest were less antibiotic. 200 $\mu \mathrm{M}$ of compounds 2.1, 2.2 and 2.7 Saccharomyces cerevisiae. Compound 2.4 showed antifungal activity at $2 \mathrm{mM}$. Structurally diverse azaphilones have been shown to be inhibitors of acetylCoA cholesterol acyltransferases, and here we demonstrate their antimicrobial properties. Of the new azaphilones tested for antimicrobial activities, compound $\mathbf{2 . 7}$ appears to be the most potent. 2.6 could not be tested due to a lack of material since it readily degraded. 
Table 2.8: Inhibition of B. subtilis, $P$. putida and $S$. cerevisiae by chaetoglobosins and azaphilones at 20 and $200 \mu \mathrm{M} .^{a}$

\begin{tabular}{|c|c|c|c|c|c|c|c|c|c|c|c|c|}
\hline Compound & \multicolumn{2}{|c|}{ (2.7) } & \multicolumn{2}{|c|}{ (2.8) } & \multicolumn{2}{|c|}{ (2.5) } & \multicolumn{2}{|c|}{ (2.4) } & \multicolumn{2}{|c|}{ (2.1) } & \multicolumn{2}{|c|}{ (2.2) } \\
\hline Assay & $20 \mu \mathrm{M}$ & $200 \mu \mathrm{M}$ & $20 \mu \mathrm{M}$ & $200 \mu \mathrm{M}$ & $20 \mu \mathrm{M}$ & $200 \mu \mathrm{M}$ & $20 \mu \mathrm{M}$ & $200 \mu \mathrm{M}$ & $20 \mu \mathrm{M}$ & $200 \mu \mathrm{M}$ & $20 \mu \mathrm{M}$ & $200 \mu \mathrm{M}$ \\
\hline P. putida & + & + & - & + & - & + & + & + & + & + & + & + \\
\hline Scerevisiae & - & + & - & - & - & - & - & - & - & + & - & + \\
\hline
\end{tabular}

+ inhibition; - no inhibition

${ }^{a}$ ANOVA, Tukey's Test, $\left.\mathrm{P}<0.05\right)$

\section{Taxonomic Significance}

This is the first comprehensive examination of metabolites produced by strains of C. globousm isolated from Canada buildings. The structures of chaetoglobosins and azaphilones isolated here were determined by high resolution mass spectrometry, NMR spectroscopy and comparison to the literature. Working with soil isolates from Nova Scotia, Brewer and Taylor (1978) reported chaetoglobosin A (2.1) and a number of other metabolites including chetomin, chaetocin and cochliodinol that were not found in these building-derived strains.

Fogle et al. (2007) studied isolates of $C$. globosum from a number of commercial laboratories that provide analysis for indoor air investigations in the U.S.A. They reported that all isolates produced chaetoglobosin $\mathrm{C}$ and half produced chaetoglobosin $\mathrm{A}$. The analysis was apparently done without authentic standards, both of which are commercially available, and was based on HPLC analysis and UV spectra for confirmation. Their C. globosum indoor isolates were grown on agar media prior to extraction for chaetoglobosin quantification. The use of agar media for metabolite screening can underestimate the toxigenic potential of fungi (Nielsen et al. 2006). It is likely that all of their isolates produced both A and C as is the case for our strains (Table 2.4) and the strains investigated by Nielsen et al. (1999). 
These strains appeared morphologically correct, had ITS sequences consistent with $C$. globosum from the NCBI database, produced predominantly chaetoglobosin A (2.1), chaetoglobosin F (2.2), chaetoglobosin C (2.3), chaetomugilin D (2.4) and chaetoviridin A (2.5) as well as other chaetoglobosins and azaphilones in minor amounts. All of the metabolites described here were also present in freeze dried media (data not shown). Few studies have reported both chaetoglobosins and azaphilones from C. globosum.

Brewer et al. (1972) reported that their Canadian strains of C. globosum produced chetomin and chaetoglobosin A (2.1). Udegawa et al. (1978) did not find chetomin in their strains of $C$. globosum from Japan but did report it in the type strain of C. subglobosum Sergeeva ATCC 14533 (CBS 149.60) isolated from dead plant material. The metabolite profiles reported here support the view of Asgari and Zare (2011) that this is likely a good species rather than conspecific with C. globosum.

Brewer et al. (1968) reported that an American soil isolate of C. globosum (HLX 819) produced cochliodinol which our strains did not although the media used were similar. Cochliodinol is moderately antifungal and antibiotic but not cytotoxic and displayed no evident toxicity in turkeys and rats (Brewer et al. 1970). This metabolite was originally reported from C. cochliodes Palliser by Safe and Taylor (1972) and detected in additional Canadian strains of that species (Brewer et al. 1968) as well as C. elatum Kunz ex Fr. (Seitka et al. 1981). In reporting production of cochliodinol by C. globosum, Brewer et al. (1968) were unable to confirm the identity of the strain which was from Minnesota. It was received as a soil culture from Prof. 
Mirocha and was sterile (Brewer et al. 1968). Based on this information and our data, it seems likely that the strain in question was misidentified. Recently, cochliodinol was identified in an extract of C. globosum when examining secondary metabolite profiles following disruption of transcriptional regulators (Nakazawa et al. 2013). The media and fermentation conditions used by these researchers were different than utilized here however they report the production of chaetoglobosin A (2.1), various chaetoviridins, 4'-epi- $N$-2-hydroxyethyl-azachaetoviridin A (2.6), aureonitol, another nitrogenated azaphilone and chaetoglobin A (Nakazawa et al. 2013).

Chaetomugilin D was also detected from all strains I studied from damp building materials in Canada. This metabolite had previous been reported from a C. globosum strain isolated from the intestinal contents of Mugil cephalus caught off the coast of Japan (Yasuhide et al. 2008). Chaetomugilin metabolites are potently cytotoxic in many human immortalized cell lines (Muroga et al. 2009). While chaetomugilin D was originally characterized by Yasuhide et al. (2008), other reports addressing this metabolite convolute the literature. Qin et al. (2009) incorrectly report the stereochemistry of C-11 as $(R)$. The physical and spectroscopic data generated agreed with that of Yasuhide et al. (2008). Our strains also produced chaetoviridin A which was originally isolated from Chaetomium globosum var. flavor-viride Novak identified by Dr. Udegawa (Takahashi et al. 1990). Some strains produced modest amounts under our growth conditions which were quite different than those of Takahashi et al. (1990) who reported using a wheat extract. Chaetoviridin A is a moderate inhibitor of a cholesteryl ester transfer protein (Tomoda et al. 1999) and is potently antifungal (Park et al. 2005).

All azaphilones isolated here had a chlorine atom at C-5 and conserved stereochemistry at the C7 and C-11 positions which is consistent with the literature. Additionally, all azaphilones 
reported here had the same 3-methyl-1-pentyl chain attached to C-3 similar to known chaetomugilins and chaetoviridins (Muroga et al. 2009; Takahashi et al. 1990). Various chains branching from the C-3 position are possible including 3-methyl-4-hydroxy-1-pentyl chains observed in some chaetomugilins (Muroga et al. 2009) and longer 3, 5-dimethyl-1,3-heptadienyl residue observed in some isochromophilones (Arai et al. 1995), luteusins, and sclerotiorin (Osmanova et al. 2010). The 3-methyl-4-hydroxy-1-pentyl chain appears prevalent in azaphilones produced by the Chaetomium genus. The latter appears to be more common in azaphilones produced by Penicillium species (Osmanova et al. 2010). Compound $\mathbf{2 . 6}$ was isolated as a nitrogenous derivative of chaetoviridin A (2.5) with an addition of an N-2 hydroxy ethyl chain at position 2 and the stereochemistry at the C-4' position is $(R)$ instead of $(S)$. Isochromophilone XIII (2.8) had the same N-2-hydroxy ethyl moiety that is also present in isochromophilone VI (Arai et al. 1995) and the cytotoxic chaetoglobin B (Ming et al. 2008). Compound 2.7 was isolated as a nitrogenated derivative of chaetoviridin E with a gammaaminobutyric acid (GABA) at position 2 as observed in isochromophilone IX (Michael et al. 2003). These are the first reported azaphilones with the 3-methyl-1-pentyl and an N-2 side chain. Recently, a biosynthetic gene cluster ( $c a z)$ was characterized in C. globosum that was demonstrated to yield both chaetomugilin and chaetoviridin azaphilones. The $65 \mathrm{~kb}$ cluster coded for both a reducing and non-reducing polyketide synthase, an acyltransferase, a halogenase, various regulatory and tailoring genes (Winter et al. 2012).

Unlike some of the other toxins discussed above, chaetoglobosins are produced by several Chaetomium species (Brewer and Taylor 1978; Udagawa et al. 1978). Under the conditions we used, chaetoglobosins A, F and $\mathrm{C}$ were dominant in our strains of C. globosum although there 
were other chaetoglobosins produced in minor amounts based on LC-UV-MS data.

Chaetoglobosin A has low toxicity to mice (Ohtsubo et al. 1978) but is potently antifungal (Amemiya et al. 1994; Betina et al. 1972), antibacterial and cytotoxic (Betina et al. 1972; Scherlach et al. 2010; Umeda et al. 1975).

Nielsen et al. (1999) examined mycotoxin production of European-derived molds on naturally and artificially infested building materials. Their C. globosum strains produced primarily chaetoglobosin $\mathrm{A}$ and $\mathrm{C}$, as well as other chaetoglobosins and unidentified compounds. An unidentified compound produced by five of six of their isolates appears to be chaetoviridin A by comparisons of the two sets of HPLC and UV data. Chaetocin, chaetomin, or sterigmatocystin were not observed in the European extracts as well. Both Canadian and European strains of $C$. globosum produce both chaetoglobosins, of which some remain unidentified, and azaphilones when grown on building materials or in liquid culture validating in situ production indoors.

In summary, a number of known $C$. globosum metabolites (2.1-2.5) including both chaetoglobosins and azaphilones, were isolated and quantified from strains obtained from Canadian building materials. Further, three new nitrogenous azaphilones $\mathbf{( 2 . 6 - 2 . 8 )}$ were also isolated and spectroscopically characterized. Of which, the newly isolated compounds, $\mathbf{2 . 6}$ and 2.8, demonstrated antibiotic activity in an in vitro assay. From these data, we found no evidence of chemotypes within C. globosum. This information has been useful in the examination of older reports of metabolites from this genus and suggests that metabolite profiles would be a useful adjunct to future taxonomic studies of this genus in addition to molecular and morphological data. 


\section{EXPERIMENTAL}

\section{C. globosum Cultures}

Chaetomium globosum isolates were obtained from Paracel Laboratories Ltd. (Ottawa, ON), Mycotaxon (Halifax, NS) and the Microfungus Collection and Herbarium from the University of Alberta (UAMH 7142, 7773). All strains were collected from indoor air samples or building materials from Ontario, Alberta, Saskatchewan, and Nova Scotia, Canada. Each of the strains from Ottawa came from different buildings in the National Capital Region (Table 2.9). Cultures were grown on malt extract agar, potato carrot agar, hay infusion agar and water agar overlaid with a sterile Whatman \#1 filter paper and incubated in the dark for 3 weeks at $25^{\circ} \mathrm{C}$. Ascocarps and ascospores were examined microscopically and were consistent with literature descriptions of this species (Domsch et al. 2007; Von Arx et al. 1986). DNA was extracted from the mycelium of each strain using an UltraClean DNA Isolation Kit (MO BIO Laboratories 12224250). PCR and sequencing was done by Laboratory Services, University of Guelph, Ontario. The PCR primers used were ITS-4 (5' TCC TCC GCT TAT TGA TAT GC 3') and ITS-1F (5' CTT GGT CAT TTA GAG GAA GTA A 3'). The PCR fragments were subsequently sequenced then aligned using MAFFT and compared to each other and the sequence of $C$. globosum using the BLASTN algorithm against the NCBI nucleotide collection database. The strains from Paracel were deposited in the National Mycological Herbarium, Ottawa; Table 2.9. All strains were aseptically transferred to sterilized 2\% malt extract (Difco) agar slants that were incubated at $25^{\circ} \mathrm{C}$ until sufficient growth was observed. After this incubation period, the metal caps on the slants were sealed with Parafilm and stored at $4^{\circ} \mathrm{C}$ until further use. 


\section{Fermentation and Metabolite Screening}

A slant of each C. globosum strain was macerated in $40 \mathrm{~mL}$ of sterile distilled deionized water and a 5\% (v/v) aliquot was utilized to inoculate three Roux bottles each containing $200 \mathrm{~mL}$

Czapek-Dox broth supplemented with $5 \%$ corn steep solids (30 g sucrose, $3 \mathrm{~g} \mathrm{NaNO}, 1 \mathrm{~g}$

$\mathrm{K}_{2} \mathrm{HPO}_{4}, 0.5 \mathrm{~g} \mathrm{MgSO}_{4}, 0.5 \mathrm{~g} \mathrm{KCl}, 0.01 \mathrm{~g} \mathrm{FeSO}_{4}, 5 \mathrm{~g}$ corn steep solids (Fermtech) per liter of ultrapure water. The resulting cultures incubated for 4 weeks in darkness at $25^{\circ} \mathrm{C}$.

Table 2.9: C. globosum strains examined for metabolite production, fungal source and ITS Genbank accession number.

\begin{tabular}{lll}
\hline Strain & Source & GenBank Accession \\
\hline DAOM 240348 & Indoor air sample, Ottawa, ON, Canada & JX130354 \\
DAOM 240349 & Indoor air sample, Ottawa, ON, Canada & JX130355 \\
DAOM 240350 & Indoor air sample, Ottawa, ON, Canada & JX130356 \\
DAOM 240351 & Indoor air sample, Ottawa, ON, Canada & JX130357 \\
DAOM 240352 & Indoor air sample, Halifax, Canada & JX130358 \\
DAOM 240353 & Indoor air sample, Ottawa, ON, Canada & JX130359 \\
DAOM 240354 & Indoor air sample, Ottawa, ON, Canada & JX130360 \\
DAOM 240355 & Indoor air sample, Ottawa, ON, Canada & \\
DAOM 240356 & Indoor air sample, Ottawa, ON, Canada & JX130361 \\
DAOM 240357 & Indoor air sample, Ottawa, ON, Canada & JX130362 \\
DAOM 240358 & Indoor air sample, Ottawa, ON, Canada & JX130363 \\
DAOM 240359 & Indoor air sample, Ottawa, ON, Canada & JX130364 \\
UAMH 7142 & Indoor air sample, Edmonton, AB, Canada & JX130366 \\
UAMH 7773 & Indoor air sample, Grimshaw, AB, Canada & JX130367 \\
DAOM 234120 & Indoor swab of building material, Guelph, ON, Canada & JX130365 \\
DAOM 242036 & Indoor air sample, Ottawa, ON, Canada & JX130345 \\
DAOM 242037 & Indoor swab of building material, Ottawa, ON, Canada & JX130346 \\
DAOM 242038 & Indoor air sample, Ottawa, ON, Canada & JX130347 \\
DAOM 242039 & Indoor air sample, Saskatoon, AB, Canada & JX130348 \\
DAOM 242040 & Indoor air sample, Ottawa, ON, Canada & JX130349 \\
DAOM 242041 & Indoor air sample, Saskatoon, AB, Canada & JX130350 \\
DAOM 242042 & Indoor air sample, Ottawa, ON, Canada & JX130351 \\
DAOM 242043 & Indoor air sample, Longueuil, QC, Canada & JX130352 \\
DAOM 242044 & Indoor air sample, Ottawa, ON, Canada & JX130353 \\
\hline
\end{tabular}

After fermentation, the fungal filtrate was separated from the cells by vacuum filtration through a

Whatman number 1 filter paper. The filtrate volumes and $\mathrm{pH}$ were recorded prior to being 
extracted twice with equal volumes of ethyl acetate. The organic layers were pooled and filtered (Whatman number 1) through anhydrous $\mathrm{Na}_{2} \mathrm{SO}_{4}$, dried by rotary evaporation, re-dissolved in ethyl acetate and transferred to a vial where they were dried under a gentle stream of nitrogen to yield crude filtrate extracts. The mycelium was wrapped in Aluminum foil, stored at $-20^{\circ} \mathrm{C}$ overnight and freeze-dried. The dried cells were weighed and extracted with ethyl acetate (100 $\mathrm{mL} \mathrm{g}^{-1}$ dry cells) overnight with constant stirring in darkness. The mycelial extract was separated from the cells by filtering (Whatman number 4) and treated as previously described. Both crude filtrate and mycelial extracts for all strains were initially screened for metabolite production by TLC $\left(0.2 \mathrm{~mm}\right.$ silica gel $60 \mathrm{~F}_{254}$ pre-coated) using $10 \%(\mathrm{v} / \mathrm{v})$ methanol in chloroform $\left(\mathrm{MeOH}-\mathrm{CHCl}_{3}\right)$. Plates were first visualized under both long $(365 \mathrm{~nm})$ and short wavelength $(254 \mathrm{~nm})$ light. Subsequently, TLC plates were dipped in ceric ammonium molybdate stain $\left(90 \mathrm{~mL} \mathrm{H}_{2} \mathrm{O}, 10 \mathrm{~mL} \mathrm{H}_{2} \mathrm{SO}_{4}, 2.5 \mathrm{~g}\right.$ ammonium molybdate, $1.0 \mathrm{~g}$ cerium (IV) sulfate) and dried with a heat gun until dark spots representing compounds were visible. Quantitative analysis of metabolites was performed using an Agilent 1100 series HPLC equipped with a quaternary pump and diode-array detector (Agilent, Mississauga, ON). Extracts were separated by a Phenomenex Gemini $\mathrm{C}_{18}(150 \mathrm{x} 4.60 \mathrm{~mm}, 3 \mu \mathrm{m}, 110 \AA)$ column (Torrance, California) using a mobile phase consisting of acetonitrile-water $\left(\mathrm{ACN}-\mathrm{H}_{2} \mathrm{O}\right)$ with trifluoroacetic acid (TFA); [0.05\%, (v:v)]. The solvent gradient was linear programmed from 5 to $100 \%$ acetonitrile over 13 minutes with a flow rate of $1.0 \mathrm{~mL} \mathrm{~min}^{-1}$. The detector was set to 210 , 254, and $400 \mathrm{~nm}$ for quantitative metabolite detection. All extracts were dissolved in HPLC grade acetonitrile and filtered through a $13 \mathrm{~mm}$ PTFE $(0.2 \mu \mathrm{m})$ membrane prior to injection. 


\section{Metabolite Isolation}

Larger scale fermentations of a high metabolite producing strain were carried out to provide adequate amounts of compounds for structural elucidation, HPLC standards and toxicity experiments. DAOM 240349 was selected based on HPLC-DAD analysis and its large filtrate extract mass. Starter cultures (50 $\mathrm{ml}$ in $250 \mathrm{ml}$ Erlenmeyer flasks) using the above media were inoculated and incubated as above. After sufficient growth of the starter cultures (approximately 1 week); they were macerated and used to inoculate Glaxo bottles each containing one liter of the same media. The filtrates from Glaxo bottles were incubated and extracted using the previously described method for the Roux bottles.

Purification of C. globosum metabolites was achieved by normal phase column chromatography followed by reverse phase semi preparative HPLC. Filtrate extracts were combined, dissolved in a minimal volume of $\mathrm{CHCl}_{3}$, and loaded onto a silica gel (40-63 $\left.\mu \mathrm{m}\right)$ column. Metabolites were fractionated using a $\mathrm{MeOH}-\mathrm{CHCl}_{3}$ gradient increasing from 0 to $10 \% \mathrm{MeOH}$ in $1 \%$ increments. Column fractions were screened by TLC as described above and similar fractions were combined. Column fractions and isolated metabolites were further purified using a Phenomenex Luna $\mathrm{C}_{18}(250 \times 10.00 \mathrm{~mm}, 5 \mu \mathrm{m}, 100 \AA)$ column (Torrance, California) and a mobile phase consisting $\mathrm{ACN}-\mathrm{H}_{2} \mathrm{O}$. The gradient was linear programmed from 10 to $100 \%$ ACN over 30 minutes for column fraction cleanup and 15 minutes for additional compound purification with a flow rate of $4 \mathrm{~mL} \mathrm{~min}^{-1}$. HPLC fractions were dried by rotary evaporation and metabolites were stored dry in amber vials. 
The dry crude fungal extract $(1.2 \mathrm{~g})$ from $10 \mathrm{~L}$ was dissolved in a minimal amount of chloroform and chromatographed on a silica gel column using a $\mathrm{CHCl}_{3}-\mathrm{MeOH}(0$ to $10 \% \mathrm{MeOH}$ in $1 \%$ increments) gradient. Column fractions were screened by TLC $\left(\mathrm{CHCl}_{3}: \mathrm{MeOH} ; 9: 1\right)$ and similar fractions were combined and dried by rotary evaporation to yield a total of 12 fractions. Column fractions of interest were further fractionated by semi preparative HPLC to yield pure metabolites. Fraction $4(57 \mathrm{mg})$ was bright yellow, eluted in 1\% MeOH, and afforded $\mathbf{2 . 5}$ (chaetoviridin A; $5.7 \mathrm{mg}$ ) and chaetomugilin D (chaetomugilin D; $26.9 \mathrm{mg}$ ). Fraction 5 (112 mg) was light yellow, eluted in 1-2\% MeOH and yielded 2.1 (chaetoglobosin A; $20.6 \mathrm{mg}$ ), 2.2 (9.6 $\mathrm{mg}$; chaetoglobosin F) and $\mathbf{2 . 3}$ (chaetoglobosin $\mathrm{C} ; 1.8 \mathrm{mg}$ ). Numerous other chaetoglobosins were in this fraction in minor amounts based on UV and MS data. $8 \% \mathrm{MeOH}$ was subdivided into fraction $8(16 \mathrm{mg})$ and fraction $9(53 \mathrm{mg})$. Fraction 8 was dark red and yielded 4'-epi- $N-2$ hydroxyethyl-azachaetoviridin A $(4.1 \mathrm{mg})$. Fraction 9 was bright orange that provided $\mathrm{N}-2$ butyric-azochaetoviridin E (6.1 mg), isochromophilone XIII $(9.8 \mathrm{mg})$, and other unidentified azaphilones in minor amounts. For semi-preparative HPLC, fractions containing azaphilones were dissolved in HPLC grade ACN whereas chaetoglobosin containing fractions were dissolved in HPLC grade $\mathrm{MeOH}$.

\section{NMR, LC-MS, UV spectroscopy and Polarimetry}

Metabolite NMR spectra were obtained on a Bruker Avance 400 Spectrometer (Milton, On) at $400.13\left({ }^{1} \mathrm{H}\right)$ and $100 \mathrm{MHz}\left({ }^{13} \mathrm{C}\right)$ using a $5 \mathrm{~mm}$ auto-tuning broadband probe with a Z-gradient. Secondary metabolites were dissolved in $\mathrm{CD}_{3} \mathrm{CN}(\delta \mathrm{H} 1.94$ and $\delta \mathrm{C} 118.7 / 1.39), \mathrm{CDCl}_{3}(\delta \mathrm{H} 7.24$ and $\delta \mathrm{C} 77.3)$ or $\mathrm{CD}_{3} \mathrm{OD}(\delta \mathrm{H} 3.30$ and $\delta \mathrm{C} 49.0)(\mathrm{CDN}$ Isotopes, Point Claire, QC) and were 
referenced to the solvent peak. Chemicals shifts were assigned based on ${ }^{1} \mathrm{H},{ }^{13} \mathrm{C},{ }^{1} \mathrm{H} /{ }^{1} \mathrm{H}$ correlation spectroscopy (COSY), heteronuclear single quantum coherence (HSQC), heteronuclear multiple bond correlation (HMBC), and distortionless enhancement by polarization transfer (DEPT-135) spectra utilizing standard Bruker pulse sequences. High resolution mass spectrometry data was acquired on a system equipped with an Agilent 1100 binary pump connected to an Agilent G1969A LC/MSD TOF HRMS. Compounds were separated on a Phenomenex Zorbax $\mathrm{C}_{18}(50 \times 3.0 \mathrm{~mm}, 1.8 \mu \mathrm{m})$ column using a mobile phase consisting of ACN- $\mathrm{H}_{2} \mathrm{O}$ with formic acid (FA); [0.1\%, (v:v)]. The gradient was linear programmed from 10 to $95 \%$ ACN over 4 minutes at a flow rate of $1 \mathrm{~mL} \mathrm{~min}^{-1}$. The MS was operated in both positive and negative ion mode. UV spectra of all major metabolites were obtained using a Varian Cary 3 UV-visible spectrophotometer scanning from 190- $800 \mathrm{~nm}$. Optical rotations were determined using an Autopol IV polarimeter (Rudolph Analytical, NJ).

\section{Calibration Plots and Recovery Studies}

Purified compounds were used to generate standard solutions in HPLC grade acetonitrile or methanol. A $5 \mathrm{mg} \mathrm{mL}^{-1}$ solution was prepared for chaetoglobosin $\mathrm{F}$, chaetoglobosin A, chaetoglobosin $\mathrm{C}$, chaetomugilin $\mathrm{D}$ and $1 \mathrm{mg} \mathrm{mL}^{-1}$ solution for chaetoviridin A. A concentration range of 0.001-5 $\mathrm{mg} \mathrm{mL}^{-1}$ and $0.0001-1 \mathrm{mg} \mathrm{mL}^{-1}$, respectively, were used for standard curves. This represents 0.01 to $50 \mu \mathrm{g}$ and 0.001 and $10 \mu \mathrm{g}$ compound on column. The calibration curves were plotted as compound peak area versus the amount of compound on column (mg) at $\lambda=254$ $\mathrm{nm}$ for chaetoglobosins and $\lambda=400 \mathrm{~nm}$ for chaetomugilin $\mathrm{D}$ and chaetoviridin A. 
For recovery studies, $50 \mathrm{~mL}$ sterile media was spiked with 1 and $10 \mu \mathrm{g}$ of chaetoglobosin A and chaetomugilin D in triplicate. The spiked media was extracted, dried, and analyzed by HPLCDAD as described. Peak identities were determined by retention time and UV full scan spectra and the recoveries were determined. Quantitative data are reported as $\mathrm{mg}$ compound $\mathrm{L}^{-1} \mathrm{~g}$ dry cell $^{-2}$ to accommodate differential growth rates of each strain and blank media was used as a negative control.

\section{Antimicrobial Testing}

The purified compounds $\mathbf{2 . 1}, \mathbf{2 . 2}, 2.4,2.5,2.7,2.8$ were tested for antimicrobial activity against Pseudomonas putida (ATCC 12633), Bacillus subtilus (ATCC 23857) and Saccharomyces cerevisae. The bacteria were grown in $5 \mathrm{~g} \mathrm{~L}^{-1}$ yeast extract, $10 \mathrm{~g} \mathrm{~L}^{-1}$ peptone and $10 \mathrm{~g} \mathrm{~L}^{-1} \mathrm{NaCl}$. S. cerevisae was grown in $1 \mathrm{~g} \mathrm{~L}^{-1}$ yeast extract supplemented with $10 \mathrm{~g} \mathrm{~L}^{-1}$ glucose. Compounds were dissolved in DMSO, individually tested at both 20 and $200 \mu \mathrm{M}$ concentrations in 96 well microplates (Nunc-Immuno MaxiSorp). A $10 \mu \mathrm{L}$ aliquot of each metabolite solution was added to $200 \mu \mathrm{L}$ of bacterial and yeast suspension. Chloramphenicol $(\sim 200 \mu \mathrm{M})$ and nystatin $(\sim 200$ $\mu \mathrm{M}$ ) were used as positive controls, respectively; whereas, DMSO was the negative control. Assays were performed in triplicate, incubated at $28^{\circ} \mathrm{C}$ with constant shaking (700 RPM), and optical density measurements were made at $600 \mathrm{~nm}$ with a Molecular Devices Spectra Max 340PC reader (Sunnyvale, CA). Readings were taken hourly for prokaryotes and every 6 hours for yeast. The data were analyzed by ANOVA followed by Tukey's test $(\mathrm{P}<0.05)$ for significant difference (Systat V13) compared to DMSO. 


\section{CHAPTER III}

ISOLATION AND STRUCTURAL ELUCIDATION OF SECONDARY METABOLITES FROM PENICILLIUM CORYLOPHILUM 


\section{INTRODUCTION}

Penicillium corylophilum Dierckx is found in soil, is somewhat xerophilic and prefers temperate climates. It is included in Raper and Thom's P. citrinum series (Raper and Thom 1949) as well as Pitt's series Citrina (Pitt 1979), however was removed from the Penicillium section Citrina by Houbraken et al. (2011) and moved to their section Exilicaulis. It is additionally not found in the related P. micznskii series by Christensen et al. (1999). This is not surprising as its morphology and secondary metabolite profiles are distinctly different compared to the tropical species included in those series. $P$. corylophilum has been reported to cause food spoilage on some low water activity foods like jams, high fat food commodities such as rapeseed or peanuts and is frequently found on cereals including barly, wheat and flour (Pitt and Hocking 2009). Samson et al. (2010) also reports it to grow on cereals, frozen fruit cakes, acid liquids and nuts mainly in temperate regions. When examining the fungal diversity associated with terrestrial plant and bird habitats in the Antarctic, P. corylophilum was the most dominant species encountered (McRae et al. 1999).

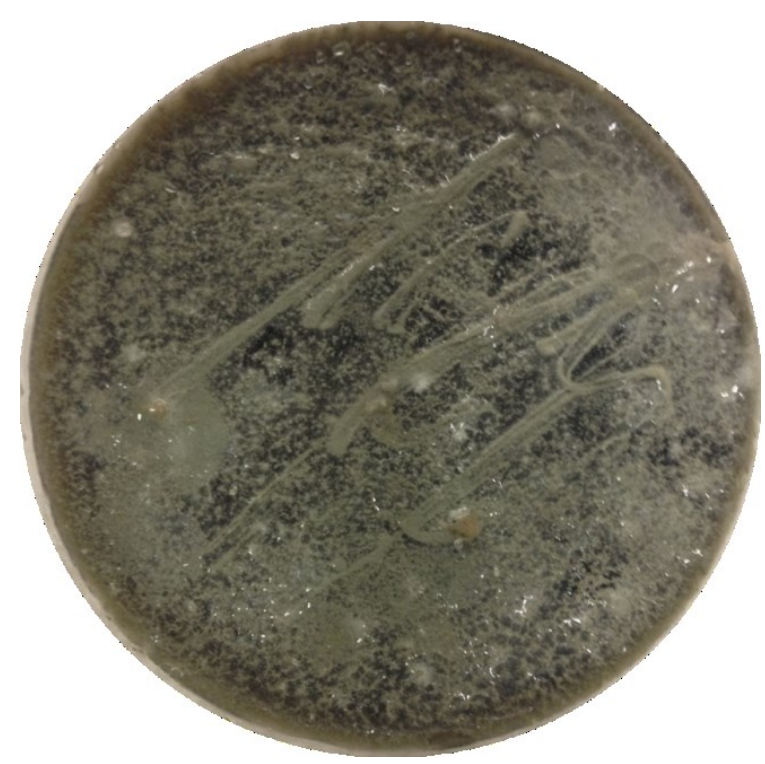

Figure 3.1: P. corylophilum growing on 2\% MEA. 
P. corylophilum is surprisingly frequently isolated from damp building materials. In a compendium of fungi isolated from moldy building materials in the USA and Canada, $P$. corylophilum was common on paper faced gypsum wallboard and was also reported from fibrous insulation, wood and manufactured wood (Miller et al. 2008). It has additionally been reported as the most frequently encountered species, $27.8 \%$ (65/234), on water damaged building materials in Northern Europe (Lian et al. 2011). P. corylophilum was found primarily on plywood, woodchip, chipboard, gypsum board, insulator wool, and flooring materials in this study. This species comprised $\sim 5 \%$ of the penicillia isolated from both damp buildings in Scandinavia and on indoor painted surfaces in Western Europe by Andersen et al. (2011) and Samson et al. (2010), respectively. It is also common in damp buildings in Japan (Ohnishi et al. 2002). In a study of moldy crawl spaces in Sweden, P. corylophilum was found to be dominant (Bok et al. 2009). These studies suggest that $P$. corylophilum will be more frequently found growing in drier indoor environments where the temperature and relative humidity fluctuate.
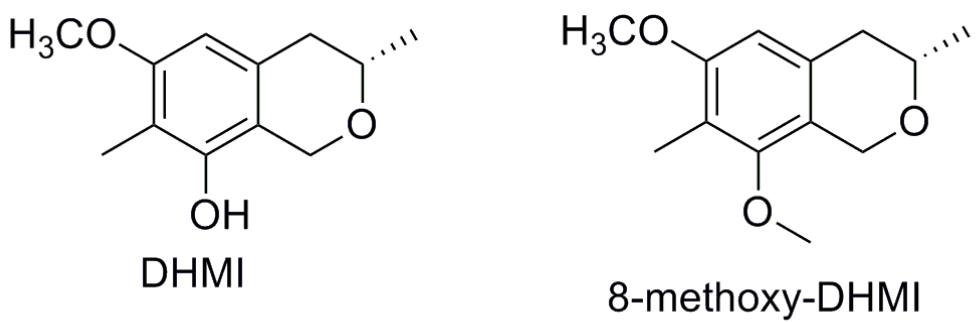

Figure 3.2: Structure of the iscohroman DHMI isolated from P. corylophilum and its synthetic derivative 8-methoxy-DHMI.

In contrast to many other Penicillium species, there are few studies of secondary metabolites of P. corylophilum. Working with a soil isolate, Cutler et al. (1989) isolated 3,7-dimethyl-8hydroxy-6-methoxyisochroman (DHMI) that exhibited plant regulatory activity. DHMI's 
synthetic derivatives, DHMI-8-acetoxy and DHMI-8-methoxy, retained biological activity and were examined in a structure activity relationship study to determine their potential agrochemical applications (figure 3.2; Cutler et al. 1997). The isocoumarins, $(+)$ orthosporin and citreoisocoumarinol, as well as the sesquiterpene phomenone were isolated from $P$. citreoviens, a synonym of $P$. corylophilum (IFO $6030=$ CBS 320.59; Lai et al. 1991; Frisvad and Filtenborg 1990). Their structures can be found in figure 3.3. Malmström et al. (2000) examined the HPLC chromatograms of ten $P$. corylophilum isolates. They were able to detect the two isocoumarins from all strains except two that were maintained too long in culture collections but could not confirm the presence of phomenone or furan-2-carboxylic acid in the extracts due to lack of reference material.

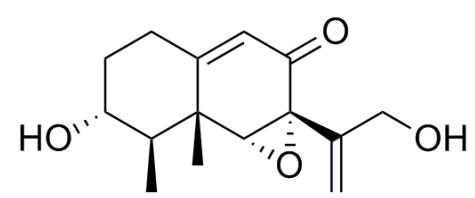

Phomeonone<smiles>CC(C)Cc1cc2cc(O)cc(O)c2c(=O)o1</smiles>

(+)-Orthosporin<smiles>CC(=O)C[C@H](C)Cc1cc2cc(O)cc(O)c2c(=O)o1</smiles>

Citreoisocoumarinol

Figure 3.3: Structure of the sesquiterpene phomenone and the isocoumarins $(+)$-orthosporin and citreoisocoumarinol reported from $P$. corylophilum.

Decarestrictines A-D that in vivo inhibit cholesterol biosynthesis and the ergot alkaloid epoxyagroclavine-I were isolated from a Portuguese soil P. corylophilum isolate (Grabley et al. 1992). More recently, an antibacterial compound, fumiquinozoline F, was characterized from the chloroform extract of a Brazilian soil P. corylophilum isolate (Silva et al. 2004) and the mycotoxin citrinin from an insect-derived strain (dos Santos et al. 2011). However, the fungal identifications for the previous chemical reports are somewhat dubious, see figure 3.4 for the structures of citrinin, epoxyagroclavine-I and decarestrictine D. Additionally, P. corylophilum 
has long been known to produce allergens leading to the serious lung disease hypersensitivity pneumonititis (Kremer et al. 1989; Ohnishi et al. 2002; Unoura et al. 2011).<smiles>CC1=C2C(=CO[C@@H](C)C2C)C(O)=C(C(=O)O)C1=O</smiles>

Citrinin<smiles>CN1C[C@]2(C)O[C@H]2C[C@@]2(C)Cc3c[nH]c4cccc(c34)C[C@H]12</smiles>

Epoxyagroclavine-I<smiles></smiles>

Decarestrictine D

Figure 3.4: Structures of citrinin, epoxyagroclavine-I and decarestrictine D previously reported to be produced by $P$. corylophilum. 


\section{RESULTS AND DISCUSSION}

P. corylophilum DAOM 242293 was isolated from a damp building in Nova Scotia and was grown in a culture conditions that favored metabolite production. The crude EtOAc extract was initially screened by LC-MS and NMR. The major components were purified from the crude extract by a combination of normal phase column chromatography, Sephadex LH20, and semipreparative reverse phase HPLC. Secondary metabolite structures were determined by analysis of NMR spectra, mass spectrometry, chemical derivatization, optical rotation, UV and comparisons to the literature. This investigation of $P$. corylophilum secondary metabolites is highlighted by the characterization of seven new compounds including four isochromans and three $\alpha$-pyrones. Additionally, three koninginins were also isolated from the EtOAc filtrate extract. These metabolites have previously been only reported from the genus Trichoderma. Two known meroterpenoids and the sesquiterpene phomenone were also isolated. Previously reported metabolites (phomenone, meroterpenoids, koninginins) were obtained from the more non-polar fractions; whereas, the new isochromans and $\alpha$-pyrones were predominantly isolated from the more polar silica gel column fractions. Metabolite production was examined from the additional eight indoor derived $P$. corylophilum strains. The fermentation, isolation, structural characterization and bioactivity of the isolated secondary metabolites are discussed here.

\section{Structural Elucidation of New Isochromans}

Compound 3.1 was isolated as a light brown oil with the molecular formula $\mathrm{C}_{17} \mathrm{H}_{24} \mathrm{O}_{7}$ determined by high resolution ESI-MS in positive mode at $m / z 323.1499\left[\mathrm{M}-\mathrm{H}_{2} \mathrm{O}+\mathrm{H}\right]^{+}$. The UV spectrum displayed absorption maxima at 250 and $307 \mathrm{~nm}$. The ${ }^{1} \mathrm{H}$ and ${ }^{13} \mathrm{C}$ NMR spectra 
revealed nineteen proton and seventeen carbon signals (Table 3.1). Examination of the mass spectrometry, NMR and UV data suggest an isochroman structure with six units of unsaturation that can be attributed to two rings and four double bonds.

The ${ }^{1} \mathrm{H}$ spectrum displayed six aliphatic methylenes between $\delta 1.27-1.57$, unequivalent methylene signals at $\delta 2.46(\mathrm{dd}, J=10.9,16.8 \mathrm{~Hz})$ and $\delta 2.55(\mathrm{dd}, J=3.5,16.8 \mathrm{~Hz})$, an oxygenated methylene at $\delta 3.54(\mathrm{t}, J=6.7 \mathrm{~Hz})$ and methine at $\delta 4.04(\mathrm{~m})$, a hemiacetal methine at $\delta 5.49$ (s), and an aromatic methine at $\delta 6.01(\mathrm{~s})$. Examination of the ${ }^{13} \mathrm{C}$ and DEPT showed that six of the seventeen carbon signals were quaternary. These signals were a result of five aromatic carbons at $\delta 103.2, \delta 112.7, \delta 141.0, \delta 161.0, \delta 162.9$, and a carbonyl at $\delta 177.9$.

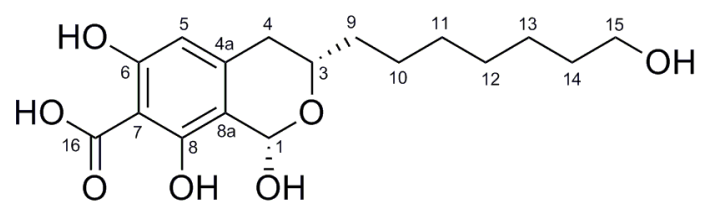

Figure 3.5: Structure of (1S,3S)-1,6,8-trihydroxy-3-(7-hydroxyheptyl) isochroman-7-carboxylic acid (3.1).

COSY correlations were observed from H-3 $(\delta 4.04)$ to $\mathrm{H}-4 \mathrm{a}(\delta 2.55), \mathrm{H}-4 \mathrm{~b}(\delta 2.46)$ and H-9 $(\delta$ 1.57) with additional correlations sequentially from $\mathrm{H}-9(\delta 1.57)$ to $\mathrm{H}-15(\delta 3.54)$. An allylic coupling was observed between the aromatic H-5 $(\delta 6.01)$ proton and one of the uniquivalent $\mathrm{H}-$ 4 resonances, $\mathrm{H}-4 \mathrm{~b}(\delta 2.46)$. The chemical shift and coupling constants of $\mathrm{H}-15,(\delta 3.54, J=6.7$ $\mathrm{Hz}$ ) suggests it is hydroxylated with a primary alcohol. An HMBC correlation from H-9 to C-3 ( $\delta$ 67.6) attaches a 7-hydroxyheptyl moiety to C-3. Both $\mathrm{H}-4 \mathrm{a}$ and $\mathrm{H}-4 \mathrm{~b}$ signals $(\delta 2.46$ and 2.55$)$ had long range correlations to $\mathrm{C}-5$ ( $\delta$ 105.6), C-4a $(\delta$ 141.0), and C-8a $\delta(112.7)$ where H-4b had 
additional HMBC correlations to C-3 ( $\delta$ 67.6) and C-9 $(\delta 36.7)$. HMBC correlation from H-5 ( $\delta$ $6.01)$ to $\mathrm{C}-4(\delta 35.4), \mathrm{C}-4 \mathrm{a}(\delta 141.0), \mathrm{C} 6(\delta 162.9), \mathrm{C}-8 \mathrm{a}(\delta 112.7)$, and $\mathrm{C}-16(\delta 177.9)$ suggest its presence in a penta-substituted aromatic ring. The hemiacetal methine H-1 ( $\delta$ 5.49) showed long range correlations with C-3 ( $\delta$ 67.6), C8 ( $\delta$ 161.0), C-4a $(\delta 141.0)$, and C-8a (112.7) suggesting C-1 and C-3 are bound by an oxygen at position 2 .

The physical, mass spectrometry, NMR and derivatization data illustrated here for compound 3.1 is consistent with a structurally similar isochroman, CJ-12,373, reported by Inagaki et al. (1998), figure 3.7. These isochromans each have two chiral centers, both at C-1 and C-3. The optical rotation of compound 3.1, $[\alpha]_{\mathrm{D}}^{25}+40.0(c 0.20, \mathrm{MeOH})$, has the same sign and magnitude compared to the structural analog CJ-12,373's optical rotation, $[\alpha]^{25}{ }_{\mathrm{D}}+32.4(c 0.13, \mathrm{MeOH})$ suggesting the same stereochemistry at C-1 and C-3. An ROE correlation between $\mathrm{H}-1 / \mathrm{H}-3$ indicates an axial symmetry and establishes the configuration at both C-1 and C-3 as $(S)$. As with CJ-12,373, the HRESIMS of compound 3.1 did not yield the parent ion, $[\mathrm{M}+\mathrm{H}]^{+}$, but rather the $\left[\mathrm{M}-\mathrm{H}_{2} \mathrm{O}+\mathrm{H}\right]^{+}$ion. This is due to the presence of a hemiacetal within compound 3.1. The same observation is made with 3.2 which also bears a hemiacetal but not 3.3 and 3.4 that lack this particular functionality. Methylation of compound 3.1 with an excess of diazomethane yielded a tetramethyl derivative, (3.5), that confirms the presence of four exchangeable acidic protons and a primary alcohol at $\mathrm{H}-15$ that was not methylated (figure 3.6). The structure of $\mathbf{3 . 1}$ was determined to be (1S,3S)-1, 6, 8-trihydroxy-3-(7-hydroxyheptyl) isochroman-7-carboxylic acid. 
<smiles>O=C(O)c1c(O)cc2c(c1O)[C@H](O)O[C@@H](CCCCCCCO)C2</smiles>

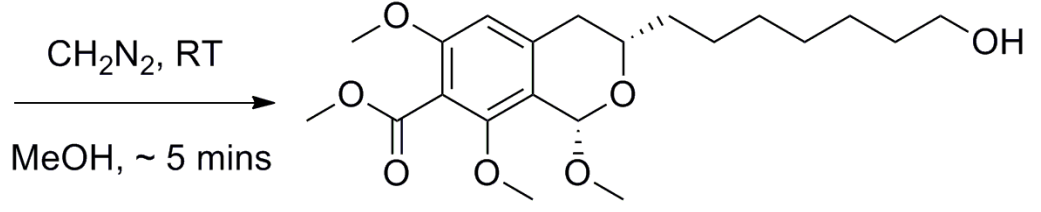

Figure 3.6: Methylation of compound (3.1) to its tetramethyl derivative (3.5) with diazomethane.<smiles>CCCCCCC[C@H]1Cc2cc(O)c(C(=O)O)c(O)c2[C@H](O)O1</smiles>

CJ-12,373

Figure 3.7: Structure of CJ- 12,373 isolated from an unidentified Penicillium species.

(1S,3S)-1,6,8-trihydroxy-3-(7-hydroxyheptyl) isochroman-7-carboxylic acid (3.1); $8.4 \mathrm{mg}$; light brown oil; $[\alpha]_{\mathrm{D}}^{25}+40.0\left(c\right.$ 0.20, MeOH); UV (MeOH)/nm $\lambda_{\max }(\log \varepsilon) 220$ (3.54), 250 (3.44), 307 (3.32); HRESIMS m/z 323.1499 [M- $\left.\mathrm{H}_{2} \mathrm{O}+\mathrm{H}\right]^{+}$(calculated for $\mathrm{C}_{17} \mathrm{H}_{23} \mathrm{O}_{6}, 323.1494$ ). ${ }^{1} \mathrm{H}$ and ${ }^{13} \mathrm{C}$ NMR data are found in table 3.1. The structure of $\mathbf{3 . 1}$ can be found in figure 3.5.

Compound 3.2 was isolated as a light brown oil with the molecular formula $\mathrm{C}_{17} \mathrm{H}_{24} \mathrm{O}_{7}$ determined by high resolution ESI-MS in positive mode at $m / z 323.1498\left[\mathrm{M}-\mathrm{H}_{2} \mathrm{O}+\mathrm{H}\right]^{+}$. The UV spectrum displayed absorption maxima at 253 and $307 \mathrm{~nm}$. The ${ }^{1} \mathrm{H}$ and ${ }^{13} \mathrm{C}$ NMR spectra revealed nineteen proton and seventeen carbon signals (Table 3.1). The mass spectrometry, NMR and UV data suggest a similar isochroman structure to compound 3.1 with six units of unsaturation that can be attributed to two rings and four double bonds. 


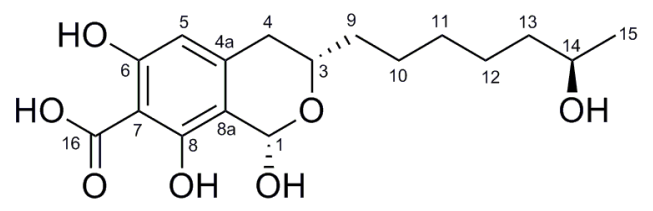

Figure 3.8: Structure of (1S,3S)-1,6,8-trihydroxy-3-((R)-6-hydroxyheptyl) isochroman-7carboxylic acid (3.2).

The 1 and 2D NMR data for compounds 3.1 and 3.2 are very similar indicating they have the same $(S)$-1,6,8-trihydroxyisochroman-7-carboxylic acid core structure. A mixture of compounds 3.1 and 3.2 produced a ${ }^{13} \mathrm{C}$ spectrum where all of the carbons in the isochroman rings had the same chemical shifts however there were small differences in the aliphatic methylene signals suggesting a structural change in the acyclic chains of the two isomers. The triplet methylene at H-15 ( $\delta 3.54)$ in compound 3.1 was replaced with an oxygenated methine multiplet at $\delta 3.71$ in compound 3.2. Additionally, compound 3.2 had five aliphatic methylenes and a doublet methyl at $\delta 1.14(\mathrm{~d}, J=6.5 \mathrm{~Hz})$ whereas compound 3.1 had six aliphatic methylenes and no methyl functionality. COSY correlations from H-14 $(\delta 3.71)$ to $\mathrm{H}-13(\delta 1.40)$ and $\mathrm{H}-15(\delta 1.14)$ establish the presence of a 6-hydroxyheptyl moiety. COSY correlations from $\mathrm{H}-3$ ( $\delta 4.04)$ to $\mathrm{H}-4 \mathrm{a}(\delta$ $2.55), \mathrm{H}-4 \mathrm{~b}(\delta \mathrm{H}-4 \mathrm{~b})$ and $\mathrm{H}-9(\delta 1.57)$ as well as an HMBC correlation from H-4b $(\delta 2.46)$ to C-9 ( $\delta$ 36.6) attach the aliphatic chain to $\mathrm{C}-3(\delta 67.6)$.

The optical rotation of compound 3.2, $[\alpha]^{25} \mathrm{D}+34.5(c 0.15, \mathrm{MeOH})$, is of the same sign and similar magnitude of compound 3.1 and CJ-12,373 (Inagaki et al. 1998). An ROE between H1/H-3 shows that they are in the same plain indicating the same $(S)$ configuration of C-1 and C-3 as both related compounds, compound $\mathbf{3 . 1}$ and $\mathrm{CJ}-12,373$. The absolute configuration of C-14 was confirmed by a modified Mosher's method where compound 3.2 was treated with both the $(R)$ - and $(S)$-MTPA chlorides to yield the corresponding esters. The differences in chemical 
shifts $\left(\Delta \delta=\delta_{S^{-}} \delta_{R}\right)$ for relevant signals determined the absolute configuration of C-14 as $(R)$, see figure 3.9. The structure of compound $\mathbf{3 . 2}$ was determined to be $(1 S, 3 S)-1,6,8$-trihydroxy-3-((R)6-hydroxyheptyl) isochroman-7-carboxylic acid.

(1S,3S)-1,6,8-trihydroxy-3-((R)-6-hydroxyheptyl) isochroman-7-carboxylic acid (3.2); $12.3 \mathrm{mg}$; light brown oil; $[\alpha]^{25}+34.5$ (c 0.15, MeOH); UV (MeOH)/nm $\lambda_{\max }(\log \varepsilon) 220$ (3.51), 253 (3.30), 307 (3.17); HRESIMS $m / z 323.1498\left[\mathrm{M}-\mathrm{H}_{2} \mathrm{O}+\mathrm{H}\right]^{+}$(calculated for $\mathrm{C}_{17} \mathrm{H}_{23} \mathrm{O}_{6}, 323.1494$ ). ${ }^{1} \mathrm{H}$ and ${ }^{13} \mathrm{C}$ NMR data are found in table 3.1. The structure of $\mathbf{3 . 2}$ can be found in figure 3.8.

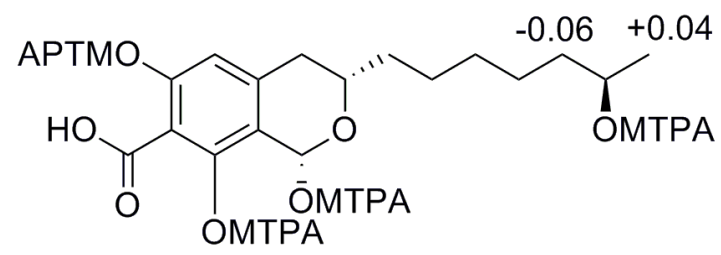

Figure 3.9: $\Delta \delta\left(\delta_{\mathrm{S}}-\delta_{\mathrm{R}}\right)$ values (in ppm) for the MTPA ester of compound 3.2.

Compound 3 was isolated a light brown oil with the molecular formula $\mathrm{C}_{17} \mathrm{H}_{24} \mathrm{O}_{6}$ determined by high resolution ESI-MS in positive at $m / z 325.1780[\mathrm{M}+\mathrm{H}]^{+}$. The UV spectrum displayed absorption maxima at 257 and $317 \mathrm{~nm}$. The ${ }^{1} \mathrm{H}$ and ${ }^{13} \mathrm{C}$ NMR spectra revealed twenty proton and seventeen carbon signals (Table 3.1). Interpretation of the NMR data and mass spectral data suggest six units of unsaturation that can be attributed to two rings and four double bonds.

The proton spectra of compounds 3.1 and 3.3 are almost identical except for the hemiacetal proton signal of 3.1 is replaced with unequivalent methylene signals at $\delta 4.50(\mathrm{~d}, J=14.7 \mathrm{~Hz})$ and $\delta 4.80(\mathrm{~d}, J=14.7 \mathrm{~Hz})$ indicating compound 3.3 lacks a hydroxyl group at this position opposed to compounds 3.1, 3.2 and CJ-12,373. COSY and HMBC data identical to compound 3.1 attach 
the same 7-hydroxyheptyl chain to C-3. The optical rotation of compound 3.3, $[\alpha]^{25}$ - -58.0 (c 0.10 MeOH), is of the opposite sign compared to compounds $\mathbf{3 . 1}$ and $\mathbf{3 . 2}$ due to the loss of a chiral center at C-1. The coupling constants (refer to Table 3.1) of H-4a and H-4b were identical for compounds 3.1-3.3, as well as the previously reported isochroman CJ-12,373. This indicates the same axial orientation of $\mathrm{H}-3$ and $(S)$ configuration at C-3 of the biosynthetically related isochromans. The structure of compounds $\mathbf{3 . 3}$ was determined to be $(S)$-6,8-dihydroxy-3-(7hydroxyheptyl) isochroman-7-carboxylic acid.

(S)-6,8-dihydroxy-3-(7-hydroxyheptyl) isochroman-7-carboxylic acid (3.3); $4.6 \mathrm{mg}$; light brown oil; $[\alpha]^{25}$ D -40.0 (c 0.15, MeOH); UV (MeOH)/nm $\lambda_{\max }(\log \varepsilon) 222$ (3.78), 257 (3.76), 317 (3.34); HRESIMS $m / z 325.1680[\mathrm{M}+\mathrm{H}]^{+}$(calcd for $\mathrm{C}_{17} \mathrm{H}_{25} \mathrm{O}_{6}, 325.1651$ ); ${ }^{1} \mathrm{H}$ and ${ }^{13} \mathrm{C}$ NMR data are found in table 3.1. The structure of $\mathbf{3 . 3}$ can be found in figure 3.10.

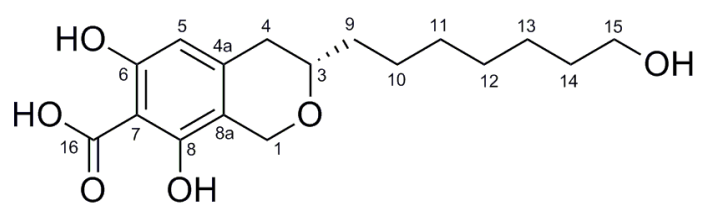

Figure 3.10: Structure of $(S)-6,8$-dihydroxy-3-(7-hydroxyheptyl) isochroman-7-carboxylic acid (3.3).

Compound 3.4 was isolated a light brown oil with the molecular formula $\mathrm{C}_{17} \mathrm{H}_{24} \mathrm{O}_{6}$ determined by high resolution ESI-MS in positive at $m / z 325.1780[\mathrm{M}+\mathrm{H}]^{+}$. The UV spectrum displayed absorption maxima at 255 and $310 \mathrm{~nm}$. The ${ }^{1} \mathrm{H}$ and ${ }^{13} \mathrm{C}$ NMR spectra revealed twenty proton and seventeen carbon signals that can be found in table 3.1. Interpretation of the NMR data and mass spectral data suggest six units of unsaturation that can be attributed to two rings and four double bonds. 
The ${ }^{1} \mathrm{H}$ and ${ }^{13} \mathrm{C}$ spectra for $\mathbf{3 . 3}$ and $\mathbf{3 . 4}$ were very similar indicating they share the same 6,8dihydroxyisochroman-7-carboxylic acid core. HMBC correlations from the aromatic H-5 ( $\delta$ 6.01) proton to $\mathrm{C}-4(\delta 35.6) \mathrm{C}-6(\delta 161.0), \mathrm{C}-7(\delta 103.0)$, and C-8a $(\delta$ 112.6) are indicative of the same pentasubstituted aromatic ring previously described. COSY correlations from $\mathrm{H}-3$ to $\mathrm{H}-4 \mathrm{a}$, H-4b and H-9 attach an aliphatic chain to H-3. The triplet methylene, observed in both compound 3.1 and 3.3, has been replaced with a multiplet methine, $\mathrm{H}-15$, and doublet methyl, $\mathrm{H}-$ 16. These chemical shifts, refer to table 3.1, and COSY correlations from $\mathrm{H}-14$ to $\mathrm{H}-13$ and $\mathrm{H}-15$ indicate the presence of an analogous heptan-2-ol chain as observed in compound 3.2. The stereochemistry of C-3 and C-15 were assigned based on the same rational as the previously described biosynthetically related isochromans. Key COSY and HMBC correlations for compound 3.4 can be found in figure 3.12. These correlations are representative of the biosynthetically related isochromans 3.1-3.4. The structure of compound 3.4 was determined to be (S)-6,8-dihydroxy-3-((R)-6-hydroxyheptyl) isochroman-7-carboxylic acid.

(S)-6,8-dihydroxy-3-((R)-6-hydroxyheptyl) isochroman-7-carboxylic acid (3.4); $3.6 \mathrm{mg}$; light brown oil; $[\alpha]^{25}{ }_{\mathrm{D}}-58.0\left(c\right.$ 0.10, MeOH); UV (MeOH)/nm $\lambda_{\max }(\log \varepsilon) 220$ (3.98), 255 (3.76), 310 (3.35); HRESIMS $m / z 325.1680[\mathrm{M}+\mathrm{H}]{ }^{+}$(calculated for $\mathrm{C}_{17} \mathrm{H}_{25} \mathrm{O}_{6}, 325.1651$ ); ${ }^{1} \mathrm{H}$ and ${ }^{13} \mathrm{C}$ NMR data are in table 3.1. The structure of $\mathbf{3 . 4}$ can be found in figure 3.11. 
Table 3.1: ${ }^{1} \mathrm{H}(700 \mathrm{MHz})$ and ${ }^{13} \mathrm{C}$ NMR Data (175 MHz) for new isochromans 3.1-3.4 in $\mathrm{CD}_{3} \mathrm{OD}$.

\begin{tabular}{|c|c|c|c|c|c|c|c|c|}
\hline \multirow[b]{2}{*}{ Position } & \multicolumn{2}{|r|}{ (3.1) } & \multicolumn{2}{|r|}{$(3.2)$} & \multicolumn{2}{|r|}{$(\mathbf{3 . 3})$} & \multicolumn{2}{|r|}{$(3.4)$} \\
\hline & $\delta_{\mathrm{C}}$ type & $\delta_{\mathrm{H}}(J, \mathrm{~Hz})$ & $\delta_{\mathrm{C}}$ type & $\delta_{\mathrm{H}}(J, \mathrm{~Hz})$ & $\delta_{\mathrm{C}}$ type & $\delta_{\mathrm{H}}(J, \mathrm{~Hz})$ & $\delta_{\mathrm{C}}$ type & $\delta_{\mathrm{H}}(J, \mathrm{~Hz})$ \\
\hline \multirow[t]{2}{*}{1} & $97.2, \mathrm{CH}$ & $5.49, \mathrm{~s}$ & $97.2, \mathrm{CH}$ & $5.49, \mathrm{~s}$ & $65.5, \mathrm{CH}$ & $4.80, \mathrm{~d}(14.7)$ & $65.5, \mathrm{CH}$ & $4.80, \mathrm{~d}(14.7)$ \\
\hline & & & & & & $4.50, \mathrm{~d}(14.7)$ & & $4.50, \mathrm{~d}(14.7)$ \\
\hline 3 & $67.6, \mathrm{CH}$ & $4.04, \mathrm{~m}$ & $67.6, \mathrm{CH}$ & $4.04, \mathrm{~m}$ & $76.0, \mathrm{CH}$ & $3.56, \mathrm{~m}$ & $76.0, \mathrm{CH}$ & $3.56, \mathrm{~m}$ \\
\hline \multirow{2}{*}{4} & $35.4, \mathrm{CH}_{2}$ & 2.55, dd $(3.5,16.8)$ & $35.4, \mathrm{CH}_{2}$ & 2.55, dd $(3.5,16.8)$ & $35.6, \mathrm{CH}_{2}$ & $2.58, \mathrm{dd}(3.5,16.5)$ & $35.6, \mathrm{CH}_{2}$ & $2.58, \mathrm{dd}(3.5,16.5)$ \\
\hline & & $2.46, \mathrm{dd}(10.9,16.8)$ & & $2.46, \mathrm{dd}(10.9,16.8)$ & & $2.50, \mathrm{dd}(10.5,16.5)$ & & $2.50, \mathrm{dd}(10.5,16.5)$ \\
\hline $4 a$ & $141.0, \mathrm{C}$ & & $141.0, \mathrm{C}$ & & $139.8, \mathrm{C}$ & & $139.8, \mathrm{C}$ & \\
\hline 5 & $105.6, \mathrm{CH}$ & $6.01, \mathrm{~s}$ & $105.6, \mathrm{CH}$ & $6.01, \mathrm{~s}$ & 106.0, $\mathrm{CH}$ & $6.01, \mathrm{~s}$ & 106.0, $\mathrm{CH}$ & $6.01, \mathrm{~s}$ \\
\hline 6 & $162.9, \mathrm{C}$ & & $162.9, \mathrm{C}$ & & $161.0, \mathrm{C}$ & & $161.0, \mathrm{C}$ & \\
\hline 7 & 103.2, C & & 103.2, C & & $103.0, \mathrm{C}$ & & $103.0, \mathrm{C}$ & \\
\hline 8 & $161.0, \mathrm{C}$ & & $161.0, \mathrm{C}$ & & $158.7, \mathrm{C}$ & & $158.7, \mathrm{C}$ & \\
\hline $8 \mathbf{a}$ & $112.7, \mathrm{C}$ & & $112.7, \mathrm{C}$ & & $112.6, \mathrm{C}$ & & $112.6, \mathrm{C}$ & \\
\hline 9 & $36.7, \mathrm{CH}_{2}$ & $1.57, \mathrm{~m}$ & $36.6, \mathrm{CH}_{2}$ & $1.57, \mathrm{~m}$ & $36.9, \mathrm{CH}_{2}$ & $1.54, \mathrm{~m}$ & $36.9, \mathrm{CH}_{2}$ & $1.54, \mathrm{~m}$ \\
\hline 10 & $26.9, \mathrm{CH}_{2}$ & $1.36, \mathrm{~m}$ & $26.8, \mathrm{CH}_{2}$ & $1.41, \mathrm{~m}$ & $26.9, \mathrm{CH}_{2}$ & $1.41, \mathrm{~m}$ & $26.8, \mathrm{CH}_{2}$ & $1.41, \mathrm{~m}$ \\
\hline 11 & $30.6, \mathrm{CH}_{2}$ & $1.27, \mathrm{~m}$ & $30.7, \mathrm{CH}_{2}$ & $1.36, \mathrm{~m}$ & $30.5, \mathrm{CH}_{2}$ & $1.35, \mathrm{~m}$ & $30.8, \mathrm{CH}_{2}$ & $1.35, \mathrm{~m}$ \\
\hline 12 & $30.7, \mathrm{CH}_{2}$ & $1.36, \mathrm{~m}$ & $26.9, \mathrm{CH}_{2}$ & $1.39, \mathrm{~m}$ & $30.7, \mathrm{CH}_{2}$ & $1.35, \mathrm{~m}$ & $26.5, \mathrm{CH}_{2}$ & $1.35, \mathrm{~m}$ \\
\hline 13 & $26.7, \mathrm{CH}_{2}$ & $1.44, \mathrm{~m}$ & $40.2, \mathrm{CH}_{2}$ & $1.40, \mathrm{~m}$ & $26.5, \mathrm{CH}_{2}$ & $1.40, \mathrm{~m}$ & $40.2, \mathrm{CH}_{2}$ & $1.40, \mathrm{~m}$ \\
\hline 14 & $33.7, \mathrm{CH}_{2}$ & $1.52, \mathrm{~m}$ & $68.5, \mathrm{CH}$ & $3.71, \mathrm{~m}$ & $33.7, \mathrm{CH}_{2}$ & $1.53, \mathrm{~m}$ & $68.5, \mathrm{CH}$ & $3.71, \mathrm{~m}$ \\
\hline 15 & $62.9, \mathrm{CH}_{2}$ & $3.54, \mathrm{t}(6.7)$ & $23.5, \mathrm{CH}_{3}$ & $1.14, \mathrm{~d}(6.5)$ & $63.0, \mathrm{CH}_{2}$ & $3.54, \mathrm{~d}(6.6)$ & $23.5, \mathrm{CH}_{3}$ & $1.14, \mathrm{~d}(6.7)$ \\
\hline 16 & $177.9, \mathrm{C}$ & & $177.9, \mathrm{C}$ & & $178.0, \mathrm{C}$ & & $178.0, \mathrm{C}$ & \\
\hline
\end{tabular}




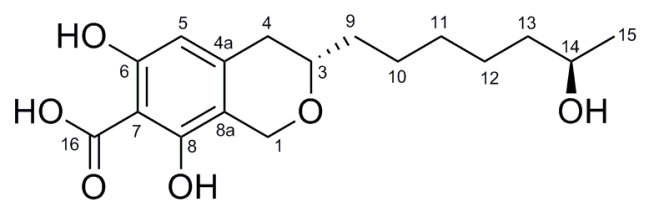

Figure 3.11: Structure of $(S)$-6,8-dihydroxy-3-((R)-6-hydroxyheptyl) isochroman-7-carboxylic acid (3.4).

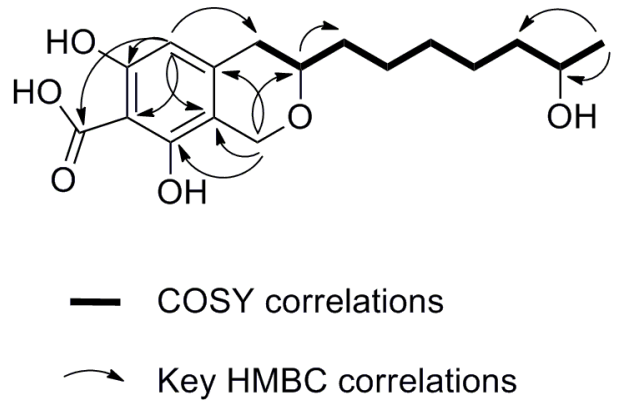

Figure 3.12: Observed COSY and key HMBC correlations for 3.4 that are representative of the four new isochromans (3.1-3.4) discussed herein.

${ }^{1} \mathrm{H},{ }^{13} \mathrm{C}$ and mass spectrometry data are presented for the tetramethyl derivative of compound $\mathbf{3 . 2}$ are presented here after the reaction mixture was cleaned up by semi-preparative HPLC (see materials and methods). (1S,3S)-methyl 3-(7-hydroxyheptyl)-1,6,8-trimethoxyisochroman-7carboxylate (3.5): $1.6 \mathrm{mg}$; clear gum; ${ }^{1} \mathrm{H}$ NMR $\left(\mathrm{CD}_{3} \mathrm{OD}, 400 \mathrm{MHz}\right) \delta 6.59(1 \mathrm{H}, \mathrm{s}), 5.50(1 \mathrm{H}, \mathrm{s})$, $4.08(1 \mathrm{H}, \mathrm{s}), 3.85(3 \mathrm{H}, \mathrm{s}), 3.80(3 \mathrm{H}, \mathrm{s}), 3.78(3 \mathrm{H}, \mathrm{s}), 3.54(2 \mathrm{H}, \mathrm{d}, J=6.5 \mathrm{~Hz}), 3.49(3 \mathrm{H}, \mathrm{s}), 2.68$ $(1 \mathrm{H}, \mathrm{m}), 2.56(1 \mathrm{H}, \mathrm{m}), 1.66-1.21(12 \mathrm{H}, \mathrm{m}) ;{ }^{13} \mathrm{C} \mathrm{NMR}\left(\mathrm{CD}_{3} \mathrm{OD}, 100 \mathrm{MHz}\right) \delta 168.6(\mathrm{C}-16), 158.8$ (C-6), 157.2 (C-8), 140.3 (C-4a), 123.1 (C-8a), 121.9 (C-8a), 117.2 (C-7), 107.4 (C-5), 96.5 (C1), 67.4 (C-3), 62.9 (C-15), 63.2, 56.5, 55.3, $52.8\left(\mathrm{O}-\mathrm{CH}_{3}\right.$ at C-1, C-6, C-8, C-16), 35.2 (C-4), 36.6 (C-9), 33.6, 30.7, 30.5, 27.0, 26.7 (C10-C14); LRESIMS m/z 365 [M-CH$\left._{3} \mathrm{OH}+\mathrm{H}\right]^{+}$ 
(calculated for $\left.\mathrm{C}_{20} \mathrm{H}_{29} \mathrm{O}_{6}, 365.1964\right)$. The structure of the tetramethyl derivative (3.5) can be found in figure 3.13 .

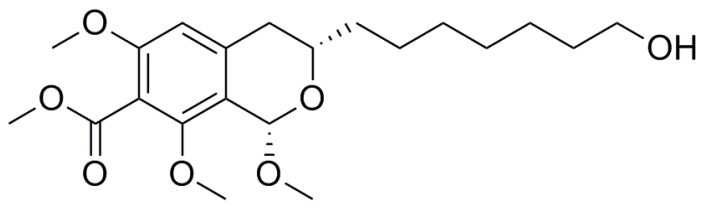

Figure 3.13: Structure of (1S,3S)-methyl 3-(7-hydroxyheptyl)-1,6,8-trimethoxyisochroman-7carboxylate (3.5), the tetramethyl derivative of 3.1 prepared by derivatization with diazomethane.

Compounds 3.1-3.4 were isolated here as new isochromans structurally similar to CJ-12,373 that was produced by an unidentified Penicillium species (Inagaki et al. 1998). These compounds all share the same 6,8-dihydroxyisochroman-7-carboxylic acid core where structural differences occur only in the acyclic moiety and at C-1. Compounds 3.1 and 3.2, as well as CJ-12,373, possess a hydroxyl group at $\mathrm{C}-1$ that is not present in 3.3 and 3.4. A 7-hydroxyheptyl moiety is attached at C-3 in both compounds 3.1 and 3.3 whereas 3.2 and 3.4 each have a 6-hydroxyheptyl chain with the $(R)$ configuration. As with CJ-12,373, HRESIMS of compounds $\mathbf{3 . 1}$ and $\mathbf{3 . 2}$ did not yield the parent ion, $[\mathrm{M}+\mathrm{H}]^{+}$, but rather the dehydrated ion, $\left[\mathrm{M}-\mathrm{H}_{2} \mathrm{O}+\mathrm{H}\right]^{+}$that is due to the labile nature of the hemiacetal functionality.

\section{Structural Elucidation of New $\alpha$-Pyrones}

Compound 3.6 was isolated as clear oil with the molecular formula $\mathrm{C}_{15} \mathrm{H}_{24} \mathrm{O}_{5}$ determined by high resolution ESI-MS in positive mode at $m / z 285.1745[\mathrm{M}+\mathrm{H}]^{+}$. The UV spectrum displayed absorption maxima at 212 and $290 \mathrm{~nm}$. The ${ }^{1} \mathrm{H}$ and ${ }^{13} \mathrm{C}$ NMR spectra revealed twenty one 
proton and fifteen carbon signals (Table 3.2). Examination of the mass spectrometry, NMR and UV data suggest a tri-substituted pyran-2-one structure with four units of unsaturation that can be attributed to one ring and three double bonds.

The ${ }^{1} \mathrm{H}$ spectrum displayed one methyl singlet at $\delta 1.83$ (s), six aliphatic methylenes between $\delta$ 1.31-1.51, unequivalent methylene signals at $\delta 2.47(\mathrm{dd}, J=8.3,14.4 \mathrm{~Hz})$ and $\delta 2.59(\mathrm{dd}, J=8.3$, $14.4 \mathrm{~Hz})$, an oxygenated methylene at $\delta 3.53(\mathrm{t}, J=7.0 \mathrm{~Hz})$, an oxygenated methine at $\delta 3.90(\mathrm{~m})$ and a deshielded methine singlet at $\delta 6.0$ (s). Examination of the ${ }^{13} \mathrm{C}$ and DEPT showed four of the fifteen carbon signals were quaternary. These were all the result of $\mathrm{sp}^{2}$ carbons at $\delta 98.7, \delta$ 161.8, $\delta 169.4$, and $\delta 170.6$.

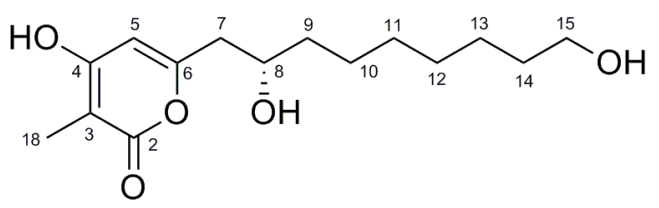

Figure 3.14: Structure of 6-((2S, 4R)-2, 4-dihydroxyundecyl)-4-hydroxy-3-methyl-2H-pyran-2one (3.6).

HMBC correlations from the singlet methyl H-16 ( $\delta$ 1.83) to C-2 $(\delta 169.4), \mathrm{C}-3(\delta 98.7)$, and C$4(\delta$ 170.6) and from the singlet methine H-5 ( $\delta$ 6.00) to C-3 $(\delta 98.7), \mathrm{C}-4(\delta 170.3), \mathrm{C}-6(\delta$ 161.8), C-7 ( $\delta$ 42.6) confirms the core of compound 3.6 is a substituted pyran-2-one. The presence of a saturated aliphatic is supported by all four units of unsaturation absorbed by the 2pyranone and the six methylenes between $\delta$ 1.31-1.51. COSY correlations were observed from $\mathrm{H}-8(\delta 3.90)$ to $\mathrm{H}-7 \mathrm{a}(\delta 2.59), \mathrm{H}-7 \mathrm{~b}(\delta 2.47)$ and $\mathrm{H}-9(\delta 1.46)$ confirming its position in the aliphatic chain. $\quad \mathrm{A}^{1} \mathrm{H}-{ }^{1} \mathrm{H}$ correlation from the oxygenated methylene triplet $\mathrm{H}-15(\delta 3.53, J=7.0$ $\mathrm{Hz})$ to $\mathrm{H}-14(\delta 1.51)$ demonstrates that end of the aliphatic chain is hydroxylated with a primary 
alcohol. HMBC correlations from both H-7a and H-7b to C-5 ( $\delta$ 104.1), C-6 $(\delta 161.8), \mathrm{C}-8(\delta$ 70.0), and C-9 ( $\delta 38.1)$ connect a 2, 9-dihydroxynonyl moiety to C-6 of the pyran-2-one ring.

The structure of $\mathbf{3 . 6}$ was determined to be a new $\alpha$-pyrone structure with a single chiral center at C-8 in the acyclic moiety. The optical rotation of 3.6, $[\alpha]^{25}-44.0(\mathrm{c}, 0.1 \mathrm{MeOH})$, is of the same sign and similar magnitude to that of the related pyrone (S)-4-hydroxy-6-(2-hydroxypropyl)-2Hpyran-2-one, $[\alpha]_{\mathrm{D}}-20.32(c$ 1.0, MeOH) (Zhou et al. 2010), where C-8 has the $(S)$ configuration. Verrucosapyrone B, $[\alpha]_{\mathrm{D}}+26.5(c 0.04, \mathrm{EtOH})\left(\right.$ Rahbaek et al. 2003), and PC-2, $[\alpha]_{\mathrm{D}}+78.5(c$ 1.0, $\mathrm{MeOH})(\mathrm{Kimura}$ et al. 1978) are structurally related $\alpha$-pyrones reported from Penicillium sp. with a single non-terminal hydroxyl in their aliphatic chains with the $(R)$ confirmation that each have a positive optical rotation. The antifungal compound, $(+)$ pulvilloric acid isolated from $P$. pulvillorum, and its $(+)$ resorcinol derivative additionally each have a single chiral center with the $(R)$ confirmation. They examined stereoselective synthesis of pulvilloric acid and unambiguously determined the $(R)$ and $(S)$ configurations for the $(+)$ and $(-)$ resorcinol derivative respectively (Rodel et al. 1996). These data indicate the confirmation of C-8 in compound 3.6 is (S) establishing the structure as $(S)$-6-(2,9-dihydroxynonyl)-4-hydroxy-3-methyl-2H-pyran-2one.

6-((2S,4R)-2, 4-dihydroxyundecyl)-4-hydroxy-3-methyl-2H-pyran-2-one (3.6); $3.0 \mathrm{mg}$; clear oil; $[\alpha]^{25}{ }_{\mathrm{D}}+8.0\left(c\right.$ 0.15, MeOH); UV (MeOH)/nm $\lambda_{\max }(\log \varepsilon) 217$ (3.74), 290 (3.72); HRESIMS $m / z$ $313.1995[\mathrm{M}+\mathrm{H}]^{+}$(calculated for $\mathrm{C}_{17} \mathrm{H}_{29} \mathrm{O}_{5}, 313.2015$ ). ${ }^{1} \mathrm{H}$ and ${ }^{13} \mathrm{C}$ NMR data are in table 3.2. The structure of $\mathbf{3 . 6}$ can be found in figure 3.14 . 
Compound 3.7 was isolated as a clear oil with the molecular formula $\mathrm{C}_{17} \mathrm{H}_{28} \mathrm{O}_{5}$ that was determined by high resolution ESI-MS in positive mode at $m / z 313.1995[\mathrm{M}+\mathrm{H}]^{+}$. The UV spectrum displayed absorption maxima at 217 and $290 \mathrm{~nm}$. The ${ }^{1} \mathrm{H}$ and ${ }^{13} \mathrm{C}$ NMR spectra revealed twenty five proton and seventeen carbon signals that can be found in table 3.2 . The mass spectrometry, NMR and UV data suggest a tri-substituted pyran-2-one structure analogous to compound 3.6 with structural differences occurring in the aliphatic chain. The same HMBC correlations observed in compound 3.6 attach a different acyclic chain C-6 of 3.7. Key HMBC and COSY correlations for 3.7 that are representative of the three new $\alpha$-pyrones discussed here can be found in figure 3.16 .

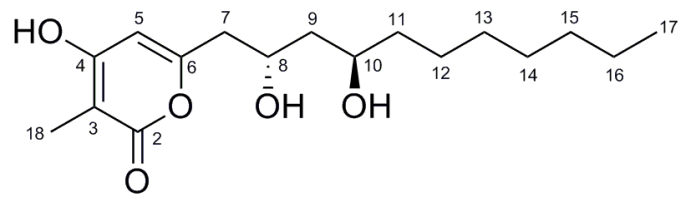

Figure 3.15: Structure of 6-((2S,4R)-2,4-dihydroxyundecyl)-4-hydroxy-3-methyl-2H-pyran-2one (3.7).

The ${ }^{1} \mathrm{H}$ spectrum of compound $\mathbf{3 . 7}$ displayed many of the same resonances observed for compound 3.6 (Table 2) but with the addition of a methyl triplet at $\delta 0.89(\mathrm{t}, J=6.8 \mathrm{~Hz})$ and another oxygenated methine at $\delta 3.78(\mathrm{~m})$. A COSY cross peak was observed between the methylene $\mathrm{H}-16(\delta 1.29)$ and the triplet methyl $\mathrm{H}-17(\delta 0.89)$ indicating the aliphatic chain terminates with a methyl instead of a primary alcohol as in compound 3.6. COSY correlations were observed sequentially through the aliphatic chain including between $\mathrm{H}-7(\delta 2.52 / 2.58), \mathrm{H}-8$ $(\delta 4.18), \mathrm{H}-9(\delta 1.52), \mathrm{H}-10(\delta 3.78)$ and H-11 $(\delta 1.41)$. These chemical shifts confirm the position of a 1,3-diol system within a 2,4-dihydroxyundecyl chain. 

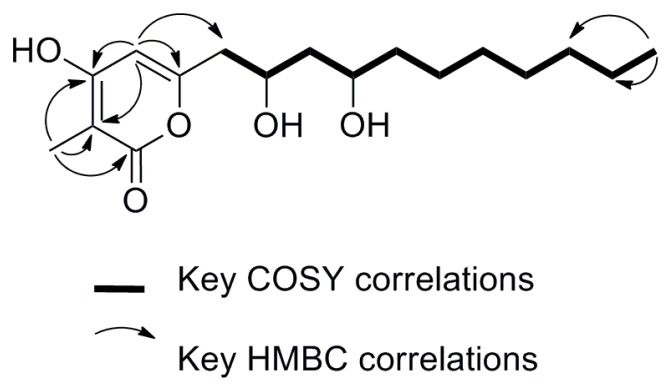

Figure 3.16: Observed COSY and key HMBC correlations for 3.7 that are representative of the three new $\alpha$-pyrones (3.6-3.8) discussed herein.

The relative configuration at C8/C10 was elucidated by Kishi's universal NMR database (Kobayashi et al. 2000). Based on this method, the stereochemical assignment of a 1,3-diol system, connected with two or more methylene bridges, can be achieved by comparison of the carbon chemical shift observed and that recorded in the database for the two only possible 1,3diol configuration (syn or anti). The comparison of the carbon chemical shift of C-10 $(\delta 69.0)$ with the reported one indicated an anti-configuration (Kobayashi et al. 2000). This establishes the structure of compound 3.7 as 6-((2S,4R)-2,4-dihydroxyundecyl)-4-hydroxy-3-methyl-2Hpyran-2-one.

6-((2S,4R)-2,4-dihydroxyundecyl)-4-hydroxy-3-methyl-2H-pyran-2-one (3.7); $3.0 \mathrm{mg}$; clear oil; $[\alpha]^{25}{ }_{\mathrm{D}}+8.0\left(c\right.$ 0.15, MeOH); UV (MeOH)/nm $\lambda_{\max }(\log \varepsilon) 217$ (3.74), 290 (3.72); HRESIMS $m / z$ $313.1995[\mathrm{M}+\mathrm{H}]^{+}$(calculated for $\left.\mathrm{C}_{17} \mathrm{H}_{29} \mathrm{O}_{5}, 313.2015\right){ }^{1} \mathrm{H}$ and ${ }^{13} \mathrm{C}$ NMR data found in table 3.2. The structure of $\mathbf{3 . 7}$ can be found in figure 3.15.

Compound 3.8 was isolated as a clear oil with the molecular formula $\mathrm{C}_{17} \mathrm{H}_{28} \mathrm{O}_{6}$ that was determined by high resolution ESI-MS in positive mode at $\mathrm{m} / z 329.1978[\mathrm{M}+\mathrm{H}]^{+}$. The UV 
spectrum displayed absorption maxima at 215 and $290 \mathrm{~nm}$. The ${ }^{1} \mathrm{H}$ and ${ }^{13} \mathrm{C}$ NMR spectra revealed twenty five proton and seventeen carbon signals (Table 3.2). Interpretation of the mass spectrometry, NMR and UV data indicate compound $\mathbf{3 . 8}$ is a tri-substituted pyran-2-one with four units of unsaturation attributed to one ring and three double bonds structurally similar to both $\alpha$-pyrones discussed above, $\mathbf{3 . 6}$ and 3.7.

The proton spectrum of compound $\mathbf{3 . 8}$ was very similar to that of compound 3.7. A single methylene was more deshielded, $\delta 1.52$ compared to $\delta 1.29$ in compound $\mathbf{6}$, and the presence of an oxygenated methylene at $\delta 3.53(\mathrm{t}, J=7.0 \mathrm{~Hz})$ replaced a triplet methyl indicating the chain terminates with an primary alcohol as in compound 5. COSY correlations were observed from sequentially from $\mathrm{H}-7$ through $\mathrm{H}-11$ establishing the same 1,3-diol found in compound 3.7. An additional COSY was observed from $\mathrm{H}-16(\delta 1.53)$ to $\mathrm{H}-17(\delta 3.54)$ confirming the aliphatic chain terminates with a primary alcohol as in compound 3.6. HMBC correlations from $\mathrm{H}-8$ to C6 and C-5 are further indicators that an undecane-1,8,10-triol chain is attached to C-6. The optical rotations of compounds 3.7 and 3.8, $[\alpha]^{25}{ }_{\mathrm{D}}+8.0(c 0.15, \mathrm{MeOH})$ and $[\alpha]^{25}{ }_{\mathrm{D}}+20.0(c$ $0.05, \mathrm{MeOH})$, are both of the same sign. Additionally, the chemical shifts of both C-8 and C-10 are identical; refer to table 2, in $\mathbf{3 . 7}$ and 3.8. Invoking the Kishi method argument used for compound 3.7, these biosynthetically related natural products have the same anti configuration of their 1,3-diol systems (Kobayashi et al. 2000). The structure of compound $\mathbf{3 . 8}$ has been established as 4-hydroxy-3-methyl-6-((2S,4R)-2,4,11-trihydroxyundecyl)-2H-pyran-2-one.

4-hydroxy-3-methyl-6-((2S,4R)-2, 4, 11-trihydroxyundecyl)-2H-pyran-2-one (3.8); $1.1 \mathrm{mg}$; clear oil; $[\alpha]^{25}{ }_{\mathrm{D}}+20.0\left(c\right.$ 0.05, MeOH); UV (MeOH)/nm $\lambda_{\max }(\log \varepsilon) 215$ (3.99), 290 (3.72); 
HRESIMS $m / z 329.1978[\mathrm{M}+\mathrm{H}]^{+}$(calculated for $\mathrm{C}_{17} \mathrm{H}_{29} \mathrm{O}_{6}, 329.1964$ ); ${ }^{1} \mathrm{H}$ and ${ }^{13} \mathrm{C}$ NMR data can be found in table 3.2. The structure of $\mathbf{3 . 8}$ can be found in figure 3.17 .

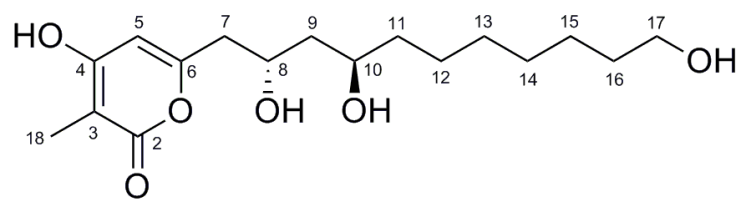

Figure 3.17: Structure of 4-hydroxy-3-methyl-6-((2S, 4R)-2, 4, 11-trihydroxyundecyl)-2Hpyran-2-one (3.8).

Compounds 3.6-3.8 are all structurally related $\alpha$-pyrones having the same pyran-2-one core with variability coming from their aliphatic chains. This is the first report of $\alpha$-pyrones from $P$. corylophilum which additionally rules out their use as indicators for fungi producing nephrotoxic ochratoxins (Rahbaek et al. 2003). $\alpha$-Pyrones are produced ubiquitously in nature and have been isolated from a wide range of fungi, plants, insects and animals (McGlaken et al. 2005). The $\alpha-$ pyrones isolated here are 4-hydroxy- $\alpha$-pyrones that have been rigorously investigated as HIV-1 protease inhibitors (Sun et al. 2005). The $\alpha$-pyrones with the same 4-hydroxy-2H-pyran-2-one core structure as compounds 3.6-3.8 elicited antimicrobial activity to Gram-positive bacteria but not Gram-negative bacteria in the low $\mu \mathrm{M}$ range. The proposed mode of action is disruption of membranes due to inhibition of fatty acid biosynthesis (Singh et al. 2003). 
Table 3.2: ${ }^{1} \mathrm{H}(700 \mathrm{MHz})$ and ${ }^{13} \mathrm{C}$ NMR Data $(175 \mathrm{MHz})$ for new $\alpha$-pyrones 3.6-3.8 in $\mathrm{CD}_{3} \mathrm{OD}$.

\begin{tabular}{|c|c|c|c|c|c|c|}
\hline \multirow[b]{2}{*}{ Position } & \multicolumn{2}{|c|}{ (3.6) } & \multicolumn{2}{|c|}{$(3.7)$} & \multicolumn{2}{|c|}{$(3.8)$} \\
\hline & $\delta_{\mathrm{C}}$, type & $\delta_{\mathrm{H}}(J, \mathrm{~Hz})$ & $\delta_{\mathrm{C}}$, type & $\delta_{\mathrm{H}}(J, \mathrm{~Hz})$ & $\delta_{\mathrm{C},}$ type & $\delta_{\mathrm{H}}(J, \mathrm{~Hz})$ \\
\hline 2 & $169.4, \mathrm{C}$ & & $169.9, \mathrm{C}$ & & $169.9, \mathrm{C}$ & \\
\hline 3 & $98.7, \mathrm{C}$ & & $98.4, \mathrm{C}$ & & $98.4, \mathrm{C}$ & \\
\hline 4 & $170.6, \mathrm{C}$ & & $172.9, \mathrm{C}$ & & $172.9, \mathrm{C}$ & \\
\hline 5 & $104.1, \mathrm{CH}$ & $6.00, \mathrm{~s}$ & $105.7, \mathrm{CH}$ & $5.97, \mathrm{~s}$ & $105.7, \mathrm{CH}$ & $5.96, \mathrm{~s}$ \\
\hline 6 & $161.8, \mathrm{C}$ & & $161.2, \mathrm{C}$ & & $161.2, \mathrm{C}$ & \\
\hline 7 & $42.6, \mathrm{CH}_{2}$ & 2.59, dd $(4.5,14.4)$ & $43.2, \mathrm{CH}_{2}$ & $2.58, \mathrm{dd}(5.3,14.5)$ & $43.2, \mathrm{CH}_{2}$ & $2.58, \mathrm{dd}(5.2,14.4)$ \\
\hline & & 2.47, dd $(8.3,14.4)$ & & 2.52, dd $(7.5,14.5)$ & & $2.54, \mathrm{dd},(7.8,14.4)$ \\
\hline 8 & $70.0, \mathrm{CH}$ & $3.90, \mathrm{~m}$ & $67.2, \mathrm{CH}$ & $4.18, \mathrm{~m}$ & $67.2, \mathrm{CH}$ & $4.19, \mathrm{~m}$ \\
\hline 9 & $38.1, \mathrm{CH}_{2}$ & $1.46, \mathrm{~m}$ & $45.3, \mathrm{CH}_{2}$ & $1.52, \mathrm{~m}$ & $45.3, \mathrm{CH}_{2}$ & $1.55, \mathrm{~m}$ \\
\hline 10 & $26.6, \mathrm{CH}_{2}$ & $1.34, \mathrm{~m}$ & $69.0, \mathrm{CH}$ & $3.78, \mathrm{~m}$ & $69.0, \mathrm{CH}$ & $3.80, \mathrm{~m}$ \\
\hline 11 & $30.5, \mathrm{CH}_{2}$ & $1.31, \mathrm{~m}$ & $39.1, \mathrm{CH}_{2}$ & $1.41, \mathrm{~m}$ & $39.1, \mathrm{CH}_{2}$ & $1.45, \mathrm{~m}$ \\
\hline 12 & $30.6, \mathrm{CH}_{2}$ & $1.34, \mathrm{~m}$ & $26.8, \mathrm{CH}_{2}$ & $1.30, \mathrm{~m}$ & $26.9, \mathrm{CH}_{2}$ & $1.46, \mathrm{~m}$ \\
\hline 13 & $26.9, \mathrm{CH}_{2}$ & $1.34, \mathrm{~m}$ & $30.8, \mathrm{CH}_{2}$ & $1.28, \mathrm{~m}$ & $30.8, \mathrm{CH}_{2}$ & $1.36, \mathrm{~m}$ \\
\hline 14 & $33.7, \mathrm{CH}_{2}$ & $1.51, \mathrm{~m}$ & $30.4, \mathrm{CH}_{2}$ & $1.28, \mathrm{~m}$ & $30.6, \mathrm{CH}_{2}$ & $1.31, \mathrm{~m}$ \\
\hline 15 & $63.0, \mathrm{CH}_{2}$ & $3.53, \mathrm{t}(7.0)$ & $33.0, \mathrm{CH}_{2}$ & $1.27, \mathrm{~m}$ & $26.8, \mathrm{CH}_{2}$ & $1.37, \mathrm{~m}$ \\
\hline 16 & $8.3, \mathrm{CH}_{3}$ & $1.83, \mathrm{~s}$ & $23.7, \mathrm{CH}_{2}$ & $1.29, \mathrm{~m}$ & $33.7, \mathrm{CH}_{2}$ & $1.53, \mathrm{~m}$ \\
\hline 17 & & & $14.4, \mathrm{CH}_{3}$ & $0.89, \mathrm{t}(6.8)$ & $63.0, \mathrm{CH}_{2}$ & $3.54, \mathrm{t}(6.7)$ \\
\hline 18 & & & $8.4, \mathrm{CH}_{3}$ & $1.83, \mathrm{~s}$ & $8.3, \mathrm{CH}_{3}$ & $1.83, \mathrm{~s}$ \\
\hline
\end{tabular}




\section{Antimicrobial Activity of New Isochromans and $\alpha$-Pyrones}

The newly characterized compounds 3.1-3.4 and 3.6-3.8 were tested for in vitro antimicrobial activity against yeast (Saccharomyces cerevisiae), as well as the Gram-positive and Gramnegative bacteria Bacillus subtilis (ATCC 23857) and Pseudomonas putida (ATCC 12633) respectively. All compounds were tested at $0.5,5$ and $50 \mu \mathrm{g} \mathrm{mL}^{-1}$ in 96 well microplates. Compounds 3.1-3.4 were additionally tested at $100 \mu \mathrm{g} \mathrm{mL}^{-1}$; compounds $\mathbf{3 . 6}$-3.8 were not tested at the highest concentration due to pure sample limitations. Compounds 3.1-3.4 significantly inhibited the growth of $S$. cerevisiae at $100 \mu \mathrm{g} \mathrm{mL}^{-1}$ (ANOVA, Tukey's Test, $\mathrm{P}<0.05$ ) compared to the negative control, DMSO. Compounds 3.1-3.4 and 3.6-3.8 were inactive at $<50$ $\mu \mathrm{g} \mathrm{mL} \mathrm{L}^{-1}$ for antimicrobial activity under the conditions tested.

\section{Previously Reported Secondary Metabolites Isolated from Indoor Strains of $\boldsymbol{P}$. corylophilum}

Phomenone (3.9); $3 \mathrm{mg}$; isolated as a light yellow powder; $[\alpha]_{\mathrm{D}}+170.7$ (c $\left.0.15, \mathrm{MeOH}\right)$; UV $(\mathrm{MeOH}) / \mathrm{nm} \lambda_{\max }(\log \varepsilon) 203$ (3.93), 240 (3.76); HRESIMS m/z 265.1436 [M+H] ${ }^{+}$(calculated for $\left.\mathrm{C}_{15} \mathrm{H}_{21} \mathrm{O}_{4}, 265.1440\right)$. The structure of phomenone was originally determined by $\mathrm{X}$ ray crystallography without NMR data Riche and Pascard-Billy (1975). ${ }^{1} \mathrm{H}$ and ${ }^{13} \mathrm{C}$ NMR data are reported in table 3.3. The ${ }^{1} \mathrm{H}$ NMR data generated here are consistent with a literature report on various derivatives of phomenone (Capasso et al. 1986). The structure of phomenone (3.9) can be found in figure 3.18 . 


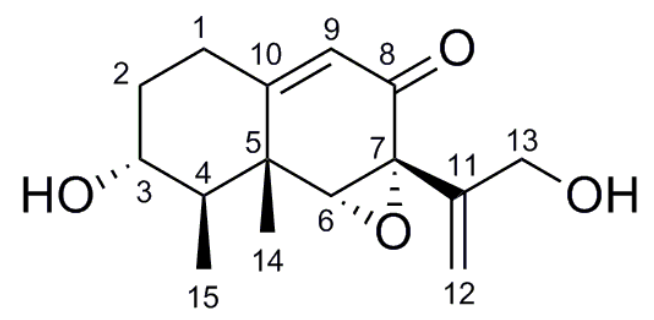

Figure 3.18: Structure of Phomenone (3.9).

Table 3.3: ${ }^{1} \mathrm{H}(400 \mathrm{MHz})$ and ${ }^{13} \mathrm{C}$ NMR Data (100 MHz) Phomenone (3.9) in $\mathrm{CD}_{3} \mathrm{OD}$.

\begin{tabular}{ccc}
\hline Position & $\delta_{\mathrm{C}}$, type & $\delta_{\mathrm{H}}(J, \mathrm{~Hz})$ \\
\hline $\mathbf{1}$ & $32.0, \mathrm{CH}_{2}$ & $2.60, \mathrm{~m}$ \\
$\mathbf{2}$ & $36.4, \mathrm{CH}_{2}$ & $2.33, \mathrm{~m}$ \\
& & $2.10, \mathrm{~m}$ \\
$\mathbf{3}$ & $71.3, \mathrm{CH}$ & $1.34, \mathrm{~m}$ \\
$\mathbf{4}$ & $46.0, \mathrm{CH}$ & $3.58, \mathrm{~m}$ \\
$\mathbf{5}$ & $42.0, \mathrm{C}$ & $1.72, \mathrm{~m}$ \\
$\mathbf{6}$ & $70.4, \mathrm{CH}$ & $3.39, \mathrm{~s}$ \\
$\mathbf{7}$ & $62.8, \mathrm{C}$ & \\
$\mathbf{8}$ & $194.6, \mathrm{C}$ & \\
$\mathbf{9}$ & $121.3, \mathrm{CH}$ & $5.71, \mathrm{~d}(1.8)$ \\
$\mathbf{1 0}$ & $166.9, \mathrm{C}$ & \\
$\mathbf{1 2}$ & $145.9, \mathrm{C}$ & $5.25, \mathrm{~m}$ \\
$\mathbf{1 3}$ & $113.3, \mathrm{CH}_{2}$ & $5.20, \mathrm{~m}$ \\
& & $4.34, \mathrm{brd}(13.8)$ \\
$\mathbf{1 4}$ & $64.0, \mathrm{CH}_{2}$ & $4.27, \mathrm{brd}(13.8)$ \\
$\mathbf{1 5}$ & $11.6, \mathrm{CH}_{3}$ & $1.30, \mathrm{~s}$ \\
& $18.8, \mathrm{CH}_{3}$ & $1.23, \mathrm{~d}(6.8)$ \\
\hline
\end{tabular}

Andrastin A (3.10); $3.4 \mathrm{mg}$; isolated as a clear gum; $[\alpha]_{\mathrm{D}}-30.0$ (c 0.10, MeOH); UV (MeOH)/nm $\lambda_{\max }(\log \varepsilon) 210$ (4.05), 284 (3.75); HRESIMS m/z $487.2723[\mathrm{M}+\mathrm{H}]^{+}$(calculated for $\mathrm{C}_{28} \mathrm{H}_{39} \mathrm{O}_{7}$ 487.2696). ${ }^{1} \mathrm{H}$ and ${ }^{13} \mathrm{C}$ NMR data were consistent with published data (Shiomi et al. 1996) and are reported in table 3.4. The structure of andrastin A (3.10) can be found in figure 3.19. 


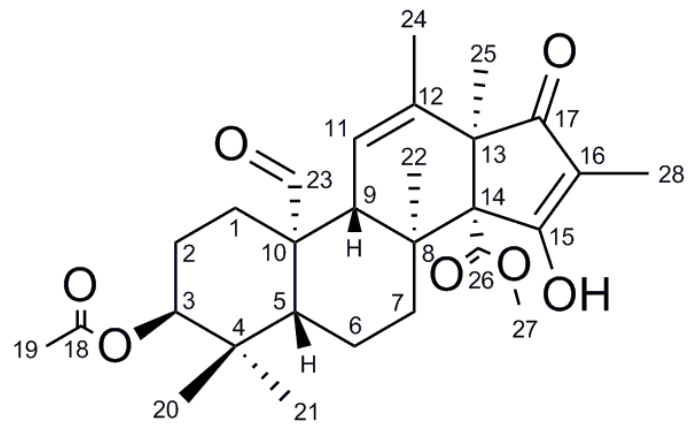

Figure 3.19: Structure of Andrastin A (3.10).

Citreohybridonol (3.11); $[\alpha]_{\mathrm{D}}+39.0(\mathrm{c} 0.20, \mathrm{MeOH}) ; \mathrm{UV}(\mathrm{MeOH}) / \mathrm{nm} \lambda_{\max }(\log \varepsilon) 205$ (3.75), 279 (3.55); HRESIMS $m / z 501.2475[\mathrm{M}+\mathrm{H}]^{+}$(calculated for $\mathrm{C}_{28} \mathrm{H}_{37} \mathrm{O}_{8}, 501.2488$ ). ${ }^{1} \mathrm{H}$ and ${ }^{13} \mathrm{C}$ NMR data were consistent with published data (Kosemura et al. 1992) and are reported in table 3.4. The structure of citreohybridinol (3.11) can be found in figure 3.20.

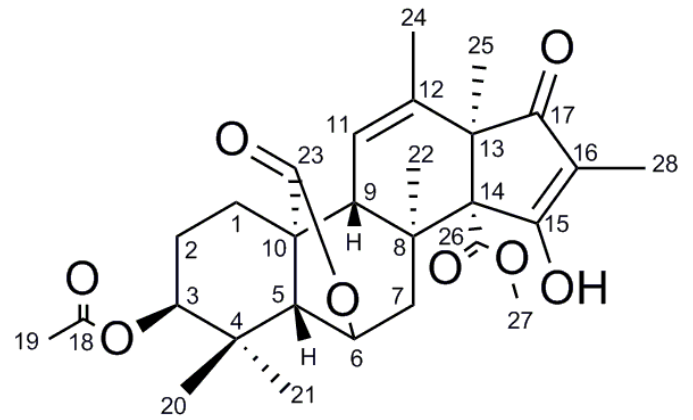

Figure 3.20: Structure of Citreohybridonol (3.11).

Koninginin A (3.12); 22.0 mg; light yellow powder; $[\alpha]_{\mathrm{D}}-33.0$ (c 0.30, MeOH); UV (MeOH)/nm $\lambda_{\max }(\log \varepsilon) 220$ (2.45), 262 (2.47); HRESIMS $m / z 285.2085[\mathrm{M}+\mathrm{H}]^{+}$(calculated for $\mathrm{C}_{16} \mathrm{H}_{29} \mathrm{O}_{4}$, 285.2066). ${ }^{1} \mathrm{H}$ and ${ }^{13} \mathrm{C}$ NMR data were consistent with published data (Cutler et al. 1989) and are reported in table 3.5. The structure of koninginin A (3.12) can be found in figure 3.21. 


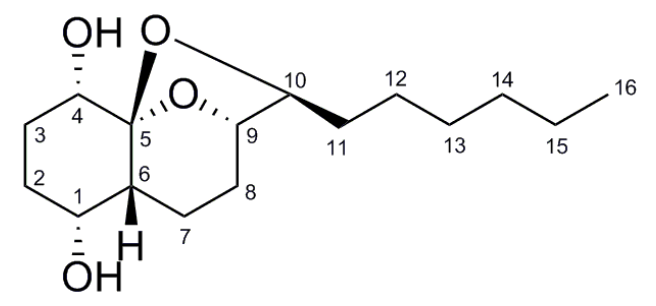

Figure 3.21: Structure of Koninginin A (3.12).

Koninginin E (3.13); $6.0 \mathrm{mg}$; isolated as a light yellow powder; $[\alpha]_{\mathrm{D}}+8.3$ (c $\left.0.30, \mathrm{MeOH}\right)$; UV $(\mathrm{MeOH}) / \mathrm{nm} \lambda_{\max }(\log \varepsilon) 205$ (2.94), 265 (3.40); HRESIMS $m / z 283.1927[\mathrm{M}+\mathrm{H}]^{+}$(calculated for $\left.\mathrm{C}_{16} \mathrm{H}_{27} \mathrm{O}_{4}, 283.1909\right) .{ }^{1} \mathrm{H}$ and ${ }^{13} \mathrm{C}$ NMR data were consistent with published data (Parker et al. 1995) and are reported in table 3.5. The optical rotation and $\delta_{\mathrm{C}}$ at position 4 distinguish koninginin $\mathrm{E}$ from its diastereomer koninginin $\mathrm{B}$. The structure of koninginin $\mathrm{E}$ (3.13) can be found in figure 3.22 .

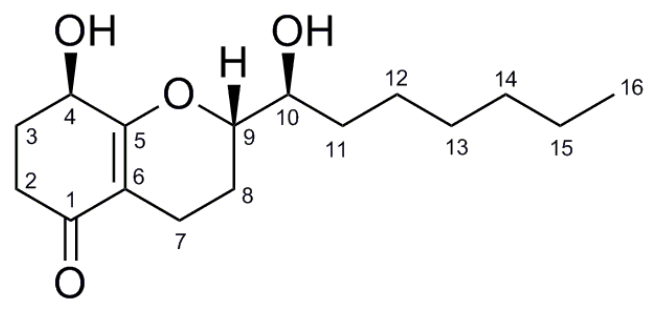

Figure 3.22: Structure of Koninginin E (3.13). 
Table 3.4: ${ }^{1} \mathrm{H}(400 \mathrm{MHz})$ and ${ }^{13} \mathrm{C}$ NMR Data $(100 \mathrm{MHz})$ for Andrastin A (3.10) and Citreohybrionol (3.11) in $\mathrm{CD}_{3} \mathrm{OD}$.

\begin{tabular}{|c|c|c|c|c|}
\hline \multirow[b]{2}{*}{ Position } & \multicolumn{2}{|c|}{ (3.10) } & \multicolumn{2}{|c|}{ (3.11) } \\
\hline & $\delta_{\mathrm{C},}$ type & $\delta_{\mathrm{H}}(J, \mathrm{~Hz})$ & $\delta_{\mathrm{C},}$ type & $\delta_{\mathrm{H}}(J, \mathrm{~Hz})$ \\
\hline \multirow[t]{2}{*}{1} & $28.9, \mathrm{CH}_{2}$ & 1.00, dd $(4.76,12.8)$ & $22.1, \mathrm{CH}_{2}$ & $1.30, \mathrm{~m}$ \\
\hline & & $2.26, \mathrm{~m}$ & & $2.10, \mathrm{~m}$ \\
\hline 2 & $24.4, \mathrm{CH}_{2}$ & $1.55, \mathrm{~m}$ & $23.1, \mathrm{CH}_{2}$ & $1.70, \mathrm{~m}$ \\
\hline 3 & $79.2, \mathrm{CH}$ & $4.60, \mathrm{t}(2.5)$ & $77.6, \mathrm{CH}$ & $4.62, \mathrm{t}(2.6)$ \\
\hline 4 & $38.1, \mathrm{C}$ & & $35.4, \mathrm{C}$ & \\
\hline 5 & $49.4, \mathrm{CH}$ & 1.86, dd $(2.6,13.3)$ & $56.1, \mathrm{CH}$ & $2.06, \mathrm{~d}(14.6)$ \\
\hline \multirow[t]{2}{*}{6} & $17.9, \mathrm{CH}_{2}$ & $1.68, \mathrm{~m}$ & $80.1, \mathrm{CH}$ & $4.83, \mathrm{~d}(3.9)$ \\
\hline & & $2.08, \mathrm{~m}$ & & \\
\hline \multirow[t]{2}{*}{7} & $33.5, \mathrm{CH}_{2}$ & $2.22, \mathrm{~m}$ & $38.1, \mathrm{CH}_{2}$ & $2.50, \mathrm{dd}(4.5,14.5)$ \\
\hline & & $3.15, \mathrm{dt}(4.1,13.3)$ & & $3.45, \mathrm{~d}(14.5)$ \\
\hline 8 & $43.0, \mathrm{C}$ & & $43.4, \mathrm{C}$ & \\
\hline 9 & $54.8, \mathrm{CH}$ & $2.17, \mathrm{bs}$ & $53.0, \mathrm{CH}$ & $2.46, \mathrm{~d}(2.5)$ \\
\hline 10 & $53.6, \mathrm{C}$ & & $45.2, \mathrm{C}$ & \\
\hline 11 & $122.8, \mathrm{CH}$ & $5.33, \mathrm{bs}$ & $122.4, \mathrm{CH}$ & $5.53, \mathrm{~s}$ \\
\hline 12 & $138.2, \mathrm{C}$ & & $140.1, \mathrm{C}$ & \\
\hline 13 & $57.4, \mathrm{C}$ & & $57.2, \mathrm{C}$ & \\
\hline 14 & $69.3, \mathrm{C}$ & & $71.8, \mathrm{C}$ & \\
\hline 15 & $192.5, \mathrm{C}$ & & $195.5, \mathrm{C}$ & \\
\hline 16 & $112.7, \mathrm{C}$ & & $113.0, \mathrm{C}$ & \\
\hline 17 & 201.7, C & & 198.1, C & \\
\hline 18 & $172.8, \mathrm{C}$ & & $172.9, \mathrm{C}$ & \\
\hline 19 & $21.1, \mathrm{CH}_{3}$ & 2.04, s & $20.9, \mathrm{CH}_{3}$ & $2.03, \mathrm{~s}$ \\
\hline 20 & $27.1, \mathrm{CH}_{3}$ & $0.93, \mathrm{~s}$ & $26.5, \mathrm{CH}_{3}$ & $0.97, \mathrm{~s}$ \\
\hline 21 & $21.6, \mathrm{CH}_{3}$ & $0.86, \mathrm{~s}$ & $22.8, \mathrm{CH}_{3}$ & $0.87, \mathrm{~s}$ \\
\hline 22 & $19.9, \mathrm{CH}_{3}$ & $1.22, \mathrm{~s}$ & $24.6, \mathrm{CH}_{3}$ & $1.29, \mathrm{~s}$ \\
\hline 23 & 207.3, CH & $10.2, \mathrm{~s}$ & $182.1, \mathrm{C}$ & \\
\hline 24 & $20.2, \mathrm{CH}_{3}$ & $1.76, \mathrm{bs}$ & $20.8, \mathrm{CH}_{3}$ & $1.85, \mathrm{bs}$ \\
\hline 25 & $16.2, \mathrm{CH}_{3}$ & $1.14, \mathrm{~s}$ & $17.9, \mathrm{CH}_{3}$ & $1.24, \mathrm{~s}$ \\
\hline 26 & $172.3, \mathrm{C}$ & & $172.2, \mathrm{C}$ & \\
\hline 27 & $52.0, \mathrm{CH}_{3}$ & $3.55, \mathrm{~s}$ & $51.7, \mathrm{CH}_{3}$ & $3.56, \mathrm{~s}$ \\
\hline 28 & $6.7, \mathrm{CH}_{3}$ & $1.55, \mathrm{~s}$ & $6.7, \mathrm{CH}_{3}$ & $1.57, \mathrm{~s}$ \\
\hline
\end{tabular}


Table 3.5: ${ }^{1} \mathrm{H}(400 \mathrm{MHz})$ and ${ }^{13} \mathrm{C}$ NMR Data (100 MHz) for koninginin A (3.12), koninginin E (3.13) and koninginin G (3.14) in $\mathrm{CD}_{3} \mathrm{OD}$.

\begin{tabular}{|c|c|c|c|c|c|c|}
\hline \multirow[b]{2}{*}{ Position } & \multicolumn{2}{|c|}{ (3.12) } & \multicolumn{2}{|r|}{ (3.13) } & \multicolumn{2}{|r|}{$(3.14)$} \\
\hline & $\delta_{\mathrm{C},}$ type & $\delta_{\mathrm{H}}(J, \mathrm{~Hz})$ & $\delta_{\mathrm{C}}$ type & $\delta_{\mathrm{H}}(J, \mathrm{~Hz})$ & $\delta_{\mathrm{C},}$ type & $\delta_{\mathrm{H}}(J, \mathrm{~Hz})$ \\
\hline 1 & $80.3, \mathrm{CH}$ & 4.25, bs & $200.3, \mathrm{C}$ & & $75.5, \mathrm{CH}$ & $3.38, \mathrm{~m}$ \\
\hline \multirow[t]{2}{*}{2} & $28.1, \mathrm{CH}_{2}$ & $1.48, \mathrm{~m}$ & 33.6 & $2.27, \mathrm{~m}$ & $33.2, \mathrm{CH}_{2}$ & $1.36, \mathrm{~m}$ \\
\hline & & $2.18, \mathrm{~m}$ & & 2.61, ddd $(4.8,9.517 .0)$ & & $1.49, \mathrm{~m}$ \\
\hline \multirow[t]{2}{*}{3} & $21.7, \mathrm{CH}_{2}$ & $1.63, \mathrm{~m}$ & $30.4, \mathrm{CH}_{2}$ & $1.98, \mathrm{~m}$ & $26.8, \mathrm{CH}_{2}$ & $1.36, \mathrm{~m}$ \\
\hline & & $2.13, \mathrm{~m}$ & & $2.16, \mathrm{~m}$ & & $1.70, \mathrm{~m}$ \\
\hline 4 & $80.5, \mathrm{CH}$ & $4.01, \mathrm{t}(6.6)$ & $66.6, \mathrm{CH}$ & $4.35, \mathrm{t}(5.0)$ & $74.8, \mathrm{CH}$ & $3.52, \mathrm{dd}(5.1,10.9)$ \\
\hline 5 & $110.1, \mathrm{C}$ & & $172.6, \mathrm{C}$ & & $98.0, \mathrm{C}$ & \\
\hline 6 & $43.1, \mathrm{CH}$ & $1.55, \mathrm{~m}$ & $112.3, \mathrm{C}$ & & $44.5, \mathrm{CH}$ & $1.45, \mathrm{~m}$ \\
\hline \multirow[t]{2}{*}{7} & $36.1, \mathrm{CH}_{2}$ & $1.46, \mathrm{~m}$ & $18.7, \mathrm{CH}_{2}$ & $2.10, \mathrm{~m}$ & $26.8, \mathrm{CH}_{2}$ & $1.49, \mathrm{~m}$ \\
\hline & & $1.60, \mathrm{~m}$ & & $2.40, \mathrm{~m}$ & & $1.79, \mathrm{~m}$ \\
\hline \multirow[t]{2}{*}{8} & $27.4, \mathrm{CH}_{2}$ & $1.83, \mathrm{~m}$ & 26.7, $\mathrm{CH}_{2}$ & $1.38, \mathrm{~m}$ & $28.7, \mathrm{CH}_{2}$ & $1.31, \mathrm{~m}$ \\
\hline & & & & $1.54, \mathrm{~m}$ & & $1.72, \mathrm{~m}$ \\
\hline 9 & $73.5, \mathrm{CH}$ & $3.84, \mathrm{~m}$ & $82.3, \mathrm{CH}$ & 3.85, ddd $(2.3,4.8,11.1)$ & $75.0, \mathrm{CH}$ & 3.78, ddd $(2.5,7.2,11.9)$ \\
\hline 10 & $70.9, \mathrm{CH}$ & $3.51, \mathrm{dd}(5.5,11.5)$ & $73.7, \mathrm{CH}$ & $3.34, \mathrm{~m}$ & $71.0, \mathrm{CH}$ & $3.66, \mathrm{~m}$ \\
\hline \multirow[t]{2}{*}{11} & $32.1, \mathrm{CH}_{2}$ & $1.58, \mathrm{~m}$ & $33.5, \mathrm{CH}_{2}$ & $1.58, \mathrm{~m}$ & $32.3, \mathrm{CH}_{2}$ & $1.58, \mathrm{~m}$ \\
\hline & & $1.77, \mathrm{~m}$ & & & & $1.78, \mathrm{~m}$ \\
\hline 12 & 26.7, $\mathrm{CH}_{2}$ & $1.31, \mathrm{~m}$ & $23.6, \mathrm{CH}_{2}$ & $1.31, \mathrm{~m}$ & $22.2, \mathrm{CH}_{2}$ & $1.52, \mathrm{~m}$ \\
\hline 13 & $30.4, \mathrm{CH}_{2}$ & $1.31, \mathrm{~m}$ & $30.6, \mathrm{CH}_{2}$ & $1.33, \mathrm{~m}$ & $30.5, \mathrm{CH}_{2}$ & $1.31, \mathrm{~m}$ \\
\hline 14 & $33.0, \mathrm{CH}_{2}$ & $1.32, \mathrm{~m}$ & $33.0, \mathrm{CH}_{2}$ & $1.32, \mathrm{~m}$ & $33.0, \mathrm{CH}_{2}$ & $1.30, \mathrm{~m}$ \\
\hline 15 & $23.7, \mathrm{CH}_{2}$ & $1.31, \mathrm{~m}$ & $23.7, \mathrm{CH}_{2}$ & $1.33, \mathrm{~m}$ & $23.7, \mathrm{CH}_{2}$ & $1.31, \mathrm{~m}$ \\
\hline 16 & $14.4, \mathrm{CH}_{3}$ & $0.91, \mathrm{t}(7.0)$ & $14.4, \mathrm{CH}_{3}$ & $0.91, \mathrm{t}(6.8)$ & $14.3, \mathrm{CH}_{3}$ & $0.90, \mathrm{t}(6.9)$ \\
\hline
\end{tabular}


Koninginin G (3.14); $5.3 \mathrm{mg}$; isolated as a light yellow powder; $[\alpha]_{\mathrm{D}}+61.3(\mathrm{c} 0.30, \mathrm{MeOH})$; UV $(\mathrm{MeOH}) / \mathrm{nm} \lambda_{\max }(\log \varepsilon) 205(2.96), 264$ (3.43); HRESIMS m/z $303.2209[\mathrm{M}+\mathrm{H}]^{+}$(calculated for $\left.\mathrm{C}_{16} \mathrm{H}_{31} \mathrm{O}_{5}, 303.2171\right) .{ }^{1} \mathrm{H}$ and ${ }^{13} \mathrm{C}$ NMR data were consistent with published data (Cutler et al. 1999) and are reported in table 3.5. The structure of $\mathbf{3 . 1 4}$ can be found in figure 3.23.

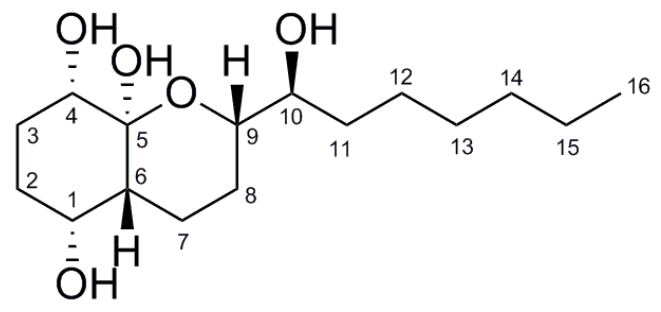

Figure 3.23: Structure of Koninginin G (3.14).

\section{Quantification of $P$. corylophilum Metabolites}

LC-UV-MS chromatograms of the extracts of the various culture conditions examined revealed that YES in still culture at $25^{\circ} \mathrm{C}$ proved to be optimal for metabolite production. The use of the other liquid media tested (2\% malt extract, Czapek-Dox) or increasing the aeration in YES cultures did not yield appreciable amounts of compounds or metabolite profiles of interest by LC-UV-MS. DAOM 242293 was selected for large scale fermentations because it readily produced a high metabolite yield after the screening of each extract by LC-UV-MS. The metabolites isolated above were utilized as analytical standards to quantify their production from the nine Canadian indoor $P$. corylophilum isolates by LC-MS when the instrument was operated in selected ion monitoring (SIM) mode, see materials and methods. Regardless of geographic origin, the strains examined produced these compounds in yields of the individual compounds from 0.5 to $>10 \mathrm{mg} \mathrm{L}^{-1}$, with an average total yield of $\sim 140 \mathrm{mg} \mathrm{L}^{-1}$. We could not detect two of 
the three $\alpha$-pyrones from DAOM 242296 and one was not detected in DAOM 242290, refer to table 3.6. The koninginins (3.12-3.14), meroterpenoids (3.10 and 3.11) and isochromans (3.13.4) were generally produced in larger amounts compared to phomenone (3.9) and the $\alpha$-pyrones (3.6-3.8). The standard curves generated for each compound had an $\mathrm{R}^{2}$ value over 0.97 over the entire concentration range. The mycotoxin citrinin was not isolated and could not be detected by LC-UV-MS in each of the nine $P$. corylophilum filtrate extracts by comparison to an authentic standard (Sigma).

\section{Taxonomic Significance}

This is the first comprehensive examination of secondary metabolites produced by $P$. corylophilum. The nine indoor derived strains were morphologically correct, had their internal transcribed spacer (ITS) sequenced and were deposited in a recognized culture collection (DAOM). Only when our strains were grown on $15 \%$ YES media did we extract appreciable amounts of secondary metabolites from the culture filtrate. Shaking the culture to increase aeration or other media did not result in production of appreciable amounts of secondary metabolites by P. corylophilum. All strains examined had the same metabolite profiles but production of compounds varied. Fractionation of the crude extract into polar and non-polar samples by normal phase silica gel chromatography yielded two fractions with different LC-UVMS patterns. The previously reported compounds phomenone (3.9), andrastin A (3.10), citreohybridonol (3.11) koninginin A (3.12), E (3.13) and G (3.14) were all found in the more non-polar EtOAc fractions with two of the new $\alpha$-pyrones (3.6 and 3.7). The new isochromans (3.1-3.4) and the other new $\alpha$-pyrone (3.8) were isolated from the more polar $20 \% \mathrm{MeOH}-$ 
Table 3.6: Metabolite production by indoor P. corylophilum strains studied.

\begin{tabular}{|c|c|c|c|c|c|c|c|c|c|c|c|c|c|}
\hline & \multicolumn{13}{|c|}{ Compound } \\
\hline DAOM & (3.9) & (3.11) & (3.10) & (3.12) & (3.13) & (3.14) & (3.6) & $(3.7)$ & (3.8) & (3.1) & $(3.2)$ & (3.3) & (3.4) \\
\hline 242288 & ++ & ++ & + & +++ & ++ & ++ & ++ & + & + & + & ++ & +++ & + \\
\hline 242289 & + & ++ & ++ & +++ & ++ & ++ & + & + & + & + & + & + & + \\
\hline 242290 & + & + & ++ & +++ & ++ & + & + & + & - & + & + & + & + \\
\hline 242291 & + & + & ++ & ++++ & + & ++ & + & + & + & + & + & + & + \\
\hline 242292 & + & ++ & + & +++ & + & + & ++ & + & + & ++ & ++ & +++ & ++ \\
\hline 242293 & + & + & + & +++ & ++ & +++ & + & + & + & ++ & ++ & +++ & + \\
\hline 242294 & + & + & ++ & ++++ & ++ & +++ & + & + & + & + & + & + & + \\
\hline 242295 & + & ++ & + & +++ & + & + & ++ & + & + & ++ & ++ & +++ & ++ \\
\hline 242296 & + & ++ & ++ & ++++ & ++ & ++++ & - & + & - & + & + & + & + \\
\hline
\end{tabular}

$+:<1 \mathrm{mg} / \mathrm{L}$

$++:>1 \mathrm{mg} / \mathrm{L},<5 \mathrm{mg} / \mathrm{L}$

$+++:>5 \mathrm{mg} / \mathrm{L},<10 \mathrm{mg} / \mathrm{L}$

$++++:>10 \mathrm{mg} / \mathrm{L}$

-: not detected 
EtOAc fraction. This chemical data agrees with the observations of Smedsgaard (1997) that YES media is a better medium compared to MEA or CYA for isochroman production.

This work confirms that this species produces phomenone (3.9) but in contrast to previous investigations, these strains did not produce the isocoumarins $(+)$ orthosporin and citreoisocoumarinol (Lai et al. 1991; Malmström et al. 2000). The latter authors were able to detect the two isocoumarins in all strains except two that were maintained for too long in culture collections but could not confirm the presence of phomenone (3.9) or furan-2-carboxylic acid due to lack of reference material. Phomenone (3.9) was isolated from a representative strain and detected from all of the P. corylophilum strains examined here by LC-MS (Table 3.6). This phytotoxin was originally isolated from the pathogenic mushroom Phoma exigua var. nonoxydabilis and has since been identified from Drechslera gigantean, Macrophomis phaseolina and a Xylaria sp. Apart from being a growth inhibitor of tomato plants causing wilting and necrosis, phomenone demonstrates antifungal, antimalarial and cytotoxicity activity (Isaka et al. 2001). Even though phomenone exhibits significant anti-plasmodial activity its cytotoxicity probably limits its applicability as an antimalarial drug. Phomenone has been studied in structure activity relationship studies with other eremophilane sesquiterpenes including PR toxin produced by P. roqueforti (Capasso et al. 1984; Moule et al. 1977). These natural products and derivatives were investigated to determine the role of the epoxide in the activity of the eremophilanes. The toxic activity of these compounds appears to be related to the existence of an aldehyde at position12. Phomenone does not have an aldehyde at this position, was found to be less toxic to mice and have less of an effect on in vitro transcription compared to PR toxin and some of its derivatives (Moule et al. 1977). Other metabolites including 
fumiquinazolin F, decarestrictines A-D, epoxyagroclavine-I, agroclavine-I, quinocitrinin A and B have been reported from P. corylophilum by Silva et al. (2004), Grabley et al. (1992) and Kozlovskii et al. (2013). The strains used in these studies were not deposited in recognized culture collections for further examination. None of these compounds or structurally similar metabolites were produced by the strains examined here.

From a taxonomic perspective, it is not surprising that isochromans $(3.1-3.4)$ were produced by P. corylophilum. Cutler et al. (1989) reported the isochroman 3,7-dimethyl-8-hydroxy-6methoxyisochroman (DHMI) as a plant growth regulator of P. corylophilum. DHMI had an $\mathrm{LD}_{50}$ of $800 \mathrm{mg} / \mathrm{kg}$ in day old chickens and inhibited etiolated wheat coleoptiles by 100 and $43 \%$ at $10^{-3}$ and $10^{-4}$ respectively. However, this isochroman was originally characterized from a $P$. steckii strain isolated from moldy millet implicated in the death of some cattle (Cox et al. 1979). DHMI and another related compound, 3,7-dimethyl-1,8-dihydroxy-6-methoxyisochroman, were characterized from the polar extract fractions of a marine P. steckii isolate (Malmström et al. 2000). These isochromans are structurally similar to the mycotoxin citrinin. Malmström et al. (2000) did not observe any citrinin in the culture filtrate extracts of ten $P$. corylophilum strains. Citrinin also was not observed in the extracts of the strains examined here, although some authors have reported citrinin from P. corylophilum (dos Santos et al. 2011; El-Kady et al. 1994). However, the isolates were not identified by Penicillium specialists and were not deposited in a culture collection. The absence of citrinin is consistent with the separation of $P$. corylophilum from citrinin-producing Penicillium species (Houbraken and Samson 2011). 
Compounds 3.1-3.4 are structurally similar to the isochroman CJ-12, 373 that was characterized from an unidentified Penicillium species. CJ-12, 373 demonstrated inhibition of prokaryotic DNA gyrase mediated supercoiling $\left(\mathrm{IC}_{50}=6 \mu \mathrm{g} \mathrm{mL}^{-1}\right)$ and relaxation $\left(\mathrm{IC}_{50}=9 \mu \mathrm{g} \mathrm{mL}^{-1}\right)$ as well as eukaryotic topoisomerase II relaxation $\left(\mathrm{IC}_{50}=1 \mu \mathrm{g} \mathrm{mL}^{-1}\right)$. Moderate antimicrobial activity $\left(\mathrm{IC}_{50}=25-100 \mu \mathrm{g} \mathrm{mL}^{-1}\right)$ against many Gram-positive bacteria attributed to inhibition of DNA gyrase was observed however it was not active against Gram positive species (Inagaki et al. 1998).

Compounds (3.6-3.8) are the first reported $\alpha$-pyrones produced by $P$. corylophilum. At least one $\alpha$-pyrone was identified by LC-MS in each strains EtOAc filtrate extract indicating all of the strains examined produce this metabolite class. The failure to detect all $\alpha$-pyrones in each strain is possibly because they were produced at low levels (Table 3.6). Rahbaek et al. (2003) characterized two new $\alpha$-pyrones, verrucosapyrone A and B, as well as two known related structures, PC-2 and LL-P888 $\gamma$ from P. verrucosum. They proposed that these $\alpha-$ pyrones, particularly PC-2 due to high production, would be good biomarkers for ochratoxin A producing fungi since all these metabolites were additionally detected in the extracts of $P$. nordicum and $P$. olsonii. Citing unpublished HPLC UV data, Rahbaek et al. (2003), report that they never observe $\alpha$-pyrones from $P$. citreovirens and $P$. citreo-viride. However, P. citreo-viride produces a wide variety of phenolics, meroterpenoids, and $\alpha$-pyrones including citreoviridin (Lai et al. 1991; Kosemura 2003). Citreoviridin is a potent inhibitor of mitochondrial ATP-synthesis/hydrolysis as are some other pyrones and meroterpenoids (Kosemura 2003; Sakabe et al. 1997). Similar $\alpha$ pyrones were reported from $P$. verrucosum and the related ochratoxin-producing species $P$. nordicum and P. olsonii (Rahbaek et al. 2003). The $\alpha$-pyrones isolated from ochratoxin- 
producing fungi were isolated from the same medium used in the present study. This suggests that different media caused differential secondary metabolites to be observed rather than taxonomic misidentifications in the past.

The meroterpenoids andrastin A (3.10) and citreohybridonol (3.11) isolated from indoor strains of $P$. corylophilum are examples of compounds arising from a mixed polyketide-terpenoid biosynthesis. These particular meroterpenoids are derived from a tertraketide moiety and 3,5dimethylorsellinic acid. No other related structures were observed in our extracts. These natural products are synthesized by plants, marine organisms and many fungal genera having diverse structures and biological activities (Kosemura 2003). Andrastin A was originally isolated from the culture broth of a hybrid strain of Penicillium sp. FO-3929 with andrastin B-C. However, it is also produced by various Penicillium species including the $P$. roqueforti complex isolated from silage (Nielsen et al. 2005), P. panum (Rasmussen et al. 2010) P. crustosum (Sonjak et al. 2005) and P. albocoremium (Overy et al. 2005). Andrastin A is a fairly potent inhibitor of farnesyl transferase (Overy et al. 2005; Shiomi et al. 1996). O'Brien et al. (2006) report upwards of 20 $\mathrm{mg} \mathrm{kg}^{-1}$ of andrastin A in bale grass silage fungal hot spots which agrees with the data reported by Rasmussen et al. (2010), $\mathrm{mg} \mathrm{kg}^{-1}$. It is also present in all blue cheeses and a high percentage (70\%) of visibly unmoldy maize silage at $0.16 \mathrm{mg} \mathrm{kg}^{-1}$ (Rasmussen et al. 2010). Recently, the andrastin biosynthetic gene cluster was identified and characterized constituting approximately $30 \mathrm{~kb}$ and 11 genes encoding a polyketide synthase, terpene cyclase and numerous tailoring enyzmes (Matsuda et al. 2013). Using Aspergillus oryzae as an expression host, andrastin A, the most complex andrastin, was obtained illustrating the potential of fungal expression systems as a means of synthesizing economically important natural products. Citreohybridonol was originally 
isolated from the mycelium of $P$. citreonigrum (formerly called $P$. citreo-viride) together with the related compounds citreohybridones $\mathrm{A}-\mathrm{B}$, isocitreohybridones $\mathrm{A}-\mathrm{B}$, and citreohybriddiones A-B (Kosemura et al. 1994). Citreohybridonol demonstrates potent anti-feedant and anti-insectal activities to the diamond black moth, Plutella xylostella, an economically important pest of cruciferous crops (Kosemura et al. 2003). These compounds exist in equilibrium between two different $\mathrm{D}$ ring tautomers and ${ }^{13} \mathrm{C}$ biosynthetic studies of citreohybridones confirm that these compounds are produced by a mixed polyketide-terpenoid pathway suggesting that andrastins are precursors of citreohybridones (Kosemura et al. 2003).

This is the first report of koninginins from any genus outside of Trichoderma. Koninginin A (3.12), E (3.13), and G (3.14) were detected from the culture filtrates of all nine strains of $P$. corylophilum, table 3.6. Other koninginins were present in these P. corylophilum extracts in minor amounts based on UV and MS data. Koninginin A, the first reported metabolite from this natural products class, was originally characterized from a strain of Trichoderma koningii isolated from a wilting plant (Cutler et al. 1989) and has since been reported from T. harzianum (Ghisalberti and Rowland 1993). Following this, koninginin E also from T. koningii (Parker et al. 1995) and koninginin G from T. aureoviride were reported (Cutler et al. 1999). Additional koninginins have been characterized from other Trichoderma spp. including T. koningii, T. pseudokoningii, T. harazium, and T. aureoviride (Reino et al. 2008). These metabolites exhibit plant growth regulatory properties by inhibiting the etiolation of wheat coleoptiles at $10^{-3} \mathrm{M}$; however, they do not possess any potent antimicrobial properties. Ghisalberti and Rowland (1993) investigated the use of antibiotic metabolites for agricultural bio-control applications. They report some modest antifungal activity for koninginins D and F against the fungus, 
Gaeumannomyces graminis var tritici that causes take all in wheat and barley (Ghisalberti and Rowland 1993). Due to the structurally similarities certain koninginins have to vitamin E and some flavonoids that inhibit phopholipase $\mathrm{A}_{2}\left(\mathrm{PLA}_{2}\right)$, they were investigated for this activity. Koninginin $\mathrm{E}$ and $\mathrm{F}$ were both efficient inhibitors of two $\mathrm{PLA}_{2}$ homologs as well as pit viper (Bothrops jararacussu) venom. Koninginin A could not inhibit either of the three which is most likely due to the presence of an additional endocyclic ring preventing interaction with the enzymes (Souza et al. 2008). Koninginins are not known for potent or broadspectrum biological activities however they may elicit amplified activity when in their proper biological niche.

This investigation of secondary metabolites produced by $P$. corylophilum strains isolated from the built environment geographically distributed across Canada has revealed a number of expected, unexpected and previously unknown compounds. The identifications of isochromans and phomenone were consistent with the literature. The report of the meroterpenoid andrastin A is consistent with unpublished data from some European strains (Dr. J. David Miller with Prof. Jens Frisvad, personal communication). The identification of the koninginins from $P$. corylophilum was unexpected, but there are other examples of secondary metabolites appearing in unrelated taxa, and these appear to be increasing in number. The use of larger scale fermentations with various culture media has produced unexpected metabolites even in very well studied Penicillium species (Nielsen et al. 2006). Penicillium corylophilum was included in the P. citrinum series by Raper and Thom (1949) and series Citrina by Pitt (1979). The metabolite data presented here, particularly the absence of citrinin, support the removal of $P$. corylophilum from the Penicillium section Citrina by Houbraken et al. (2011) to their section Exilicaulis.

\section{EXPERIMENTAL}




\section{P. corylophilum Cultures}

Penicillium corylophilum Dierckx isolates were obtained from Paracel Laboratories Ltd.

(Ottawa, Ontario) and were all collected directly from building materials or indoor air samples (Table 3.7). Cultures were grown on 2\% malt extract agar (MEA) and Czapek yeast extract agar and microscopic features were consistent with literature descriptions of this species (Samson et al. 2010). DNA was extracted from the mycelium of each strain using an UltraClean DNA Isolation Kit (MO BIO Laboratories 12224-250). PCR amplification and sequencing of the ITS1, 5.8S and ITS2 genes were performed by the National Fungal Identification Service, Ottawa, Ontario. The PCR primers used were UN-Up18S42 (5' - CGTAACAAGGTTTCCGTAGGT GAAC-3') and UN-Lo28S22 (5' -GTTTCTTTTCCTCCGCTTATTGATATG-3').The PCR fragments were sequenced, aligned using MAFFT and subsequently compared to each other and the sequence of $P$. corylophilum using the BLASTN algorithm against the NCBI nucleotide collection database. The identifications were confirmed by the identification service at the National Mycological Herbarium (Agriculture Agri-Food Canada, Ottawa) and deposited with the Canadian Collection of Fungal Cultures, DAOM; refer to table 3.7. All strains were aseptically transferred to sterile $2 \%$ malt extract agar plates and slants. These were incubated at $25^{\circ} \mathrm{C}$ until sufficient growth was observed. After this incubation period, plates were sealed with Parafilm and stored at $4^{\circ} \mathrm{C}$ until required.

\section{Fermentation and Metabolite Screening}


A slant of P. corylophilum DAOM 242291, randomly selected, was macerated in sterile distilled deionized water and an aliquot was utilized to inoculate $(5 \%, v / v)$ five Roux bottles each containing $200 \mathrm{~mL}$ of yeast extract sucrose broth (YES; $20 \mathrm{~g} / \mathrm{L}$ yeast extract [Difco], $150 \mathrm{~g} / \mathrm{L}$ sucrose, $\left.0.5 \mathrm{~g} / \mathrm{L} \mathrm{MgSO}_{4} \cdot 7 \mathrm{H}_{2} \mathrm{O}\right)$, malt extract broth (20 g/L malt extract [Difco]), or CzapekDox broth $\left(30 \mathrm{~g} / \mathrm{L}\right.$ sucrose, $3 \mathrm{~g} / \mathrm{L} \mathrm{NaNO}_{3}, 1 \mathrm{~g} / \mathrm{L} \mathrm{K}_{2} \mathrm{HPO}_{4}, 0.5 \mathrm{~g} / \mathrm{L} \mathrm{MgSO}_{4} \cdot 7 \mathrm{H}_{2} \mathrm{O}, 0.5 \mathrm{~g} / \mathrm{L} \mathrm{KCl}$, $0.01 \mathrm{~g} / \mathrm{L} \mathrm{FeSO}_{4} \cdot 7 \mathrm{H}_{2} \mathrm{O}, 5 \mathrm{~g} / \mathrm{L}$ yeast extract). Cultures were incubated at $25^{\circ} \mathrm{C}$ in the dark. To determine the time course for metabolite production, one Roux bottle was extracted at one week intervals. Fernbach flasks containing $560 \mathrm{~mL}$ of YES were inoculated $(5 \%, \mathrm{v} / \mathrm{v})$ and incubated in shake culture to examine the effect of aeration on metabolite production (NB Scientific triple tier shaker, $3.5 \mathrm{~cm}$ throw, $220 \mathrm{rpm}$ ).

Table 3.7: Indoor P. corylophilum strains examined for metabolite production, location, fungal source and GenBank accession.

\begin{tabular}{cccc}
\hline Strain & Location & Source & GenBank Accession \\
\hline DAOM 242288 & Ottawa, ON & Indoor air sample & KF170363 \\
DAOM 242289 & Ottawa, ON & Indoor air sample & KF170358 \\
DAOM 242290 & Ottawa, ON & Indoor air sample & KF170357 \\
DAOM 242291 & Montreal, QC & Building materials & KF170364 \\
DAOM 242292 & Calgary, AB & Indoor air sample & KF170361 \\
DAOM 242293 & Halifax, NS & Building materials & KF170356 \\
DAOM 242294 & Ottawa, ON & Indoor air sample & KF170362 \\
DAOM 242295 & Calgary, AB & Indoor air sample & KF170360 \\
DAOM 242296 & Victoria, BC & Indoor air sample & KF170359 \\
\hline
\end{tabular}

The liquid cultures were filtered (Whatman \#4) and the volume of the culture filtrate and $\mathrm{pH}$ were measured. The mycelium was frozen and stored in the freezer at $-20 \mathrm{C}$. The culture filtrates were saturated with sodium chloride, exhaustively extracted with ethyl acetate and filtered 
(Whatman \#1) through anhydrous sodium sulfate prior to being dried by rotary evaporation. The dried crude extract was re-dissolved in a minimal amount of HPLC grade methanol, filtered through a $13 \mathrm{~mm}$ PTFE $0.2 \mu \mathrm{m}$ filter, and dried under a gentle stream of nitrogen gas.

The dry P. corylophilum extracts were each dissolved in $1 \mathrm{~mL}$ of HPLC grade methanol and analyzed by liquid chromatography-ultra violet-mass spectrometry (LC-UV-MS). This was achieved using a Waters 2795 separations module, Waters 996 diode array detector, and Waters MicroMass Quattro LC mass spectrometer. Compounds were separated by a Phenomenex Kinetex $\mathrm{C}_{18}(100 \times 4.60 \mathrm{~mm}, 2.6 \mu \mathrm{m}, 100 \AA)$ column (Torrance, California) using a mobile phase consisting of ACN- $\mathrm{H}_{2} \mathrm{O}$ with FA; $[0.1 \%,(\mathrm{v} / \mathrm{v})]$. The solvent gradient was linear programmed

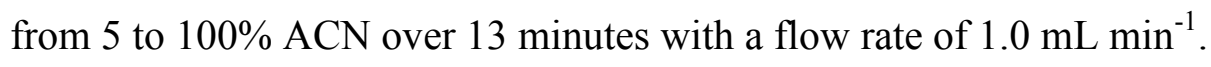

Based on the UV and mass spectrometry data of the representative extracts, the optimal medium and fermentation time were determined for metabolite production by $P$. corylophilum DAOM 242291. All nine strains were subsequently inoculated as above into three Roux bottles containing $200 \mathrm{~mL}$ of sterile YES media and grown in stationary culture for two weeks in the dark at $25 \mathrm{C}$. The culture filtrate and cells were treated as above.

Calibration plots for compounds (3.1-3.4, 3.6-3.14) were constructed using the same LC conditions described above for screening extracts; however, the gradient was linear programmed for 20 minutes instead of 13. The MS was operated in selected ion monitoring (SIM) mode using the $[\mathrm{M}+\mathrm{H}]^{+}$ion for all compounds except 3.1 and 3.2 where the dehydrated ion, $\left[\mathrm{M}-\mathrm{H}_{2} \mathrm{O}+\mathrm{H}\right]^{+}$, was utilized. Each standard solution was analyzed in triplicate and the calibration plot was 
represented by $0.1,1.0$, and $10 \mu \mathrm{g}$ purified compound on the column. The extracts were screened by LC-MS for the production of citrinin by reference to an authentic standard (Sigma).

\section{Metabolite Isolation}

Larger scale fermentations of $P$. corylophilum DAOM 242293 were done to produce sufficient quantities of metabolites for structural elucidation and toxicity assays. The optimal fermentation conditions determined during the screening process (YES liquid media, $25^{\circ} \mathrm{C}$, stationary in the dark) were used for larger scale fermentations in Glaxo bottles. Twenty $250 \mathrm{~mL}$ Erlenmeyer flasks containing $50 \mathrm{~mL}$ of sterile YES liquid media were inoculated $(5 \% \mathrm{v} / \mathrm{v})$ and incubated using conditions that favored metabolite production until sufficient growth was observed ( $\sim 3$ days). Starter cultures were macerated, individually transferred to Glaxo bottles containing $1 \mathrm{~L}$ of the same sterile media and left for two weeks under the same fermentation conditions described above.

After fermentation, the culture filtrate was separated from the mycelia by vacuum filtration (Whatman \#4), saturated with $\mathrm{NaCl}$, exhaustively extracted with ethyl acetate (EtOAc), filtered (Whatman \#1) through anhydrous $\mathrm{Na}_{2} \mathrm{SO}_{4}$ and dried by rotary evaporation. The crude filtrate extracts were pooled, taken up in a minimal amount of methanol and dried under a gentle stream of nitrogen. 
The crude EtOAc extract (5.2 g) was subjected to vacuum liquid chromatography on a short silica gel column using a step gradient elution with hexanes-EtOAc (0-100\%) in 20\% increments followed by EtOAc-MeOH (0-100\%) in 10\% increments. The 100\% EtOAc fraction (1.5 g) was further fractionated by flash column chromatography on silica gel (40-63 $\mu \mathrm{m})$ using a MeOH$\mathrm{CHCl}_{3}$ gradient that increased from 0 to $10 \% \mathrm{MeOH}-\mathrm{CHCl}_{3}$ in $1 \%$ increments to yield nine fractions. Fraction 2 eluted in $1 \% \mathrm{MeOH}$ and yielded phomenone, 3.9, $(3.0 \mathrm{mg})$ as well as the meroterpenoids andrastin A, 3.10, (3.4 mg) and citreohybridinol, 3.11, (5.3 mg). Fraction 4 eluted in 3\% MeOH-CHCl 3 and yielded koninginin A, 3.12, (22.0 mg), E, 3.13, (6.0 mg) and G, 3.14, $(5.3 \mathrm{mg})$ as well as compound $3.6(2.3 \mathrm{mg})$. Fraction 7 eluted in $7 \% \mathrm{MeOH}-\mathrm{CHCl}_{3}$ and provided compound $3.7(3.0 \mathrm{mg})$. Both fractions 4 and 7 were further purified by reverse phase (RP) semi-preparative HPLC using a Phenomenex Luna $C_{18}(250 \times 10.00 \mathrm{~mm}, 5 \mu \mathrm{m}, 100 \AA)$ column to yield the mentioned metabolites. The linear gradient was programmed from $10-80 \%$ ACN- $\mathrm{H}_{2} \mathrm{O}$ over 19 minutes with a flow rate of $4 \mathrm{ml} \mathrm{min}^{-1}$. Compound $\mathbf{3 . 7}$ was obtained from fraction 7 eluting in $70 \% \mathrm{ACN}-\mathrm{H}_{2} \mathrm{O}$ and compound $\mathbf{3 . 6}$ was purified from fraction 4 eluting in $56 \% \mathrm{ACN}-\mathrm{H}_{2} \mathrm{O}$.

The 50\% EtOAc-MeOH (3.2 g) fraction from the step gradient was applied to a silica gel column and washed with $10 \% \mathrm{MeOH}-\mathrm{CHCl}_{3}$ to remove less polar components and further fractionated by a $\mathrm{MeOH}-\mathrm{CHCl}_{3}$ gradient using $10 \%$ increments. The $20 \% \mathrm{MeOH}-\mathrm{CHCl}_{3}$ fraction $(1.5 \mathrm{~g})$ was subjected to Sephadex $\mathrm{LH}-20$ using $100 \% \mathrm{MeOH}$ as the mobile phase, yielding nine fractions. The first three fractions were pooled and applied to a silica gel column using a $\mathrm{MeOH}-\mathrm{CHCl}_{3}$ gradient increasing from 0 to $10 \% \mathrm{MeOH}-\mathrm{CHCl}_{3}$ in $1 \%$ increments, yielding three fractions. Fraction 2 was further purified by RP semi-preparative HPLC using a Whatman Partisil 10 ODS- 
$3 \mathrm{C}_{18}(250 \times 9.40 \mathrm{~mm}, 10 \mu \mathrm{m})$ and a linear gradient programmed from 5 to $45 \% \mathrm{ACN}-\mathrm{H}_{2} \mathrm{O}$ over 19 minutes. This afforded compound $3.8(1.1 \mathrm{mg})$ that eluted in $49 \% \mathrm{ACN}-\mathrm{H}_{2} \mathrm{O}$. The other six Sephadex fractions were spectroscopically the same and were fractionated by RP semipreparative HPLC using the same methods as described for compound 3.8. Compounds 3.1 (8.4 $\mathrm{mg}$ ), 3.2 (12.3 mg), 3.3 (4.6) and $\mathbf{3 . 4}\left(3.6 \mathrm{mg}\right.$ ) eluted in 58, 60, 69, and 71\% $\mathrm{ACN}-\mathrm{H}_{2} \mathrm{O}$, respectively.

\section{Methylation of 3.1 with Diazomethane}

Compound 3.1 $(4.0 \mathrm{mg})$ was dissolved in $500 \mu \mathrm{L}$ of HPLC grade methanol and an excess of diazomethane was added at room temperature. The reaction mixture was stirred until nitrogen gas liberation halted. The reaction mixture was dried under a gentle stream of nitrogen and the methylated product was cleaned up by semi-preparative reverse phase HPLC to yield $\mathbf{3 . 5}$, as previously described above for compounds 3.1-3.4.

\section{Preparation of the $(R)$ - and $(S)$-MTPA Esters of compound 3.2}

Compound $3.2(1.5 \mathrm{mg})$ was dissolved in $500 \mu \mathrm{L}$ of dry pyridine. $2 \mathrm{mg}$ of $\mathrm{N}$, N-dimethyl-4aminopyridine (DMAP) and $10 \mu \mathrm{L}$ of $(S)$-MTPACl were added. The reaction mixture was stirred at room temperature overnight. The reaction was quenched with $1 \mathrm{~mL}$ of distilled deionized $\mathrm{H}_{2} \mathrm{O}$ and extracted with EtOAc (3 $x 2 \mathrm{~mL})$. The organic phase was washed with $2 \mathrm{~mL}$ of $5 \%$ $\mathrm{NaHCO}_{3}$, passed through anhydrous $\mathrm{Na}_{2} \mathrm{SO}_{4}$ and dried under a gentle stream of nitrogen to yield 
the $(S)$-MTPA ester of compound 3.2. By the same methods, the $(R)$-MTPA ester of compound 3.2 was also obtained.

\section{General Experimental Procedures}

NMR experiments $\left({ }^{1} \mathrm{H},{ }^{13} \mathrm{C}\right.$, HSQC, HMBC, COSY, DEPT, ROESY) were performed on a Bruker Avance III $700 \mathrm{MHz}$ NMR spectrometer equipped with a $5 \mathrm{~mm}$ QNP cryoprobe and operating at $700.17 \mathrm{MHz}$ for ${ }^{1} \mathrm{H}$ and 176.06 $\mathrm{MHz}$ for ${ }^{13} \mathrm{C}$ or Bruker Avance 400 Spectrometer (Milton, On) at $400.13\left({ }^{1} \mathrm{H}\right)$ and $100 \mathrm{MHz}\left({ }^{13} \mathrm{C}\right)$ using a $5 \mathrm{~mm}$ auto-tuning broadband probe with a Z-gradient. Compounds were dissolved in $\mathrm{CD}_{3} \mathrm{OD}$ (CDN Isotopes, Point Claire, Quebec) and referenced to the solvent peak $(\delta \mathrm{H} 3.31$ or $\delta \mathrm{C} 49.1)$. HRESIMS spectra were obtained on a QStar high resolution mass spectrometer. Semi-preparative HPLC was achieved using an Agilent 1100 HPLC system equipped with a diode array detector and compounds were separated by either a Whatman Partisil 10 ODS-3 $\mathrm{C}_{18}(250$ x $9.40 \mathrm{~mm}, 10 \mu \mathrm{m})$ or Phenomenex Luna $\mathrm{C}_{18}(250$ x $10.00 \mathrm{~mm}, 5 \mu \mathrm{m}, 100 \AA$ ) column. Column fractions were screened by LC-UV-MS using a Waters 2795 separations module, Waters 996 diode array detector, and Micromass Quatro LC mass spectrometer. Fractions were separated by a Phenomenex Kinetix $\mathrm{C}_{18}(100 \mathrm{x} 4.60 \mathrm{~mm}$, 2.6 $\mu \mathrm{m}, 100 \AA$ ) column (Torrance, California) using a mobile phase consisting of ACN- $\mathrm{H}_{2} \mathrm{O}$ with formic acid (FA); $[0.1 \%,(\mathrm{v} / \mathrm{v})]$. The solvent gradient was linear programmed from 5 to $100 \%$ ACN over 13 minutes with a flow rate of $1.0 \mathrm{ml} \mathrm{min}^{-1}$. Optical rotations were measured using an Autopol IV polarimeter (Rudolph Analytical, NJ). UV spectra were obtained on a Varian Cary 3 UV-visible spectrophotometer scanning from 800-190 nm. silica gel (Silicycle; 
40-63 $\mu \mathrm{m})$, Sephadex LH-20 (GE) and TLC ( $0.2 \mathrm{~mm}$ silica gel $60 \mathrm{~F}_{254}$ pre-coated) plates were used in the isolation of metabolites.

\section{Antimicrobial Assays}

Compounds 3.1-3.4, 3.6-3.8 were tested for in vitro antimicrobial activity against Pseudomonas putida (ATCC 12633), Bacillus subtilis (ATCC 23857) and Saccharomyces cerevisiae. Bacteria were inoculated and grown in $5 \mathrm{~g} \mathrm{~L}^{-1}$ yeast extract, $10 \mathrm{~g} \mathrm{~L}^{-1}$ peptone and $10 \mathrm{~g} \mathrm{~L}^{-1} \mathrm{NaCl} . S$.

cerevisiae was inoculated and grown in $1 \mathrm{~g} \mathrm{~L}^{-1}$ yeast extract supplemented with $10 \mathrm{~g} \mathrm{~L}^{-1}$ glucose. Compounds were individually tested at $0.5,5$ and $50 \mu \mathrm{g} \mathrm{mL}^{-1}$ in 96 well microplates (NuncImmuno MaxiSorp). Compounds 3.1-3.4 were additionally tested at $100 \mu \mathrm{g} \mathrm{mL}^{-1}$ due to metabolite availability. Compounds were dissolved in DMSO and a $10 \mu \mathrm{L}$ aliquot of each individual metabolite solution was added to $200 \mu \mathrm{L}$ of bacterial and yeast suspension. Chloramphenicol $\left(50 \mu \mathrm{g} \mathrm{mL}^{-1}\right)$ and nystatin $\left(100 \mu \mathrm{g} \mathrm{mL}^{-1}\right)$ were used as respective positive controls whereas DMSO was the negative control. Assays were performed in triplicate and incubated at $28^{\circ} \mathrm{C}$ with constant shaking (700 RPM). Optical density (OD) measurements were made at $600 \mathrm{~nm}$ with a Molecular Devices Spectra Max 340PC reader (Sunnyvale, CA) taken hourly for bacteria and every four hours for yeast. Antimicrobial OD data were analyzed by ANOVA followed by Tukey's test $(\mathrm{P}<0.05)$ for significant growth differences (Systat V13) compared to the negative control (DMSO). 
CHAPTER IV

CHARACTERIZATION OF METABOLITES FROM WALLEMIA SEBI 


\section{INTRODUCTION}

The genus Wallemia has been taxonomically placed in the phylum Basidiomycota and is comprised of three species: W. sebi, W. muriae, and W. ichthyophaga (Zalar et al. 2005). This genus was previously described by mycologists as halophilic; however, it is xerophilic as growth within this phylogenetic grouping is independent of the solute used to increase the osmotic pressure of the medium (Pitt and Hocking 2009; Wheeler et al. 1988). Xerophilicity within the Basidiomycetes is rare. However, phylogenetic analysis of both nuclear small subunit ribosomal DNA and ITS 1 and 2 regions including the 5.8S rDNA supports the placement of this genus within this taxonomic group (Matheny et al. 2006; Padamesee et al. 2012; Zalar et al. 2005).

$W$. sebi is a primary colonizer that grows when the $a_{w}$ of the substrate is below 0.80 . The term water activity, $a_{w}$, refers to the amount of biologically available water within a substrate. This is defined as the ratio of the vapour pressure exerted by water in the (hygroscopic or porous) material to the vapour pressure of pure water at the same temperature and pressure. The $\mathrm{a}_{\mathrm{w}}$ can be determined indirectly by using a dew point meter to measure the relative humidity of the atmosphere within a sealed container in which a sample of the material has been allowed to equilibrate (Flannigan and Miller 2011). This species is capable of sporulation within one or two days when grown on media with $a_{w}$ values between 0.99-0.91 and within five days when the $a_{w}$ was 0.85 at $25^{\circ} \mathrm{C}$ (Pitt and Hocking 2009). W. sebi is capable of growth over a wide $a_{w}$ range, 0.69 to 0.99 , at $25^{\circ} \mathrm{C}$ in glucose or fructose media. It grows slowly on higher $a_{w}$ media such as CYA or MEA whereas the other two currently recognized Wallemia species, $W$. muriae and $W$. ichthyophaga, cannot grow on these media (Pitt and Hocking 2009; Zalara et al. 2005). W. sebi 
has been shown to grow very slowly when there is no solute within the growth medium (Kuncic et al. 2010). Figure 4.1 depicts $W$. sebi growing on MEA.

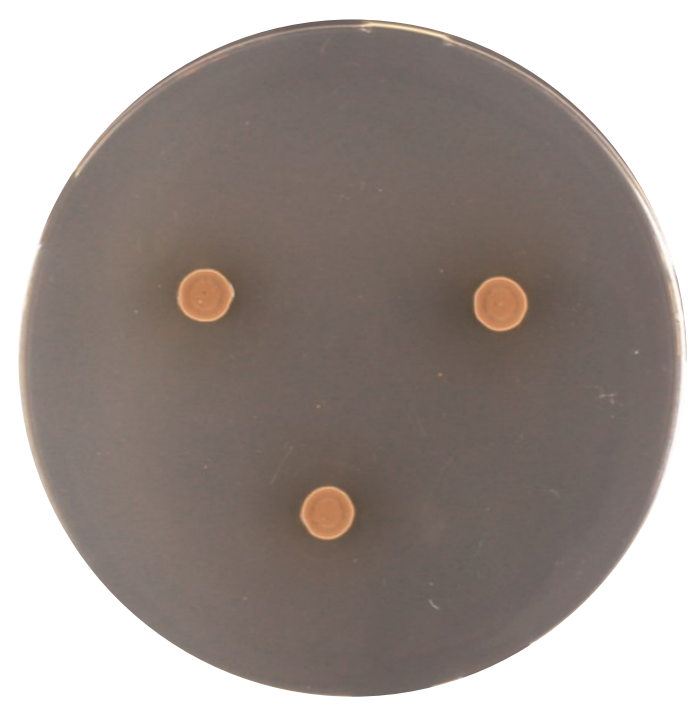

Figure 4.1: W. sebi growing on 2\% Malt Extract Agar.

W. sebi is cosmopolitan; however, it is easily overlooked because it forms small, dull brown colonies that would typically be overgrown by other fungi on conventional growth media (Botic et al. 2012; Wood et al. 1990). More than a century ago, it was studied because it grew on and spoiled dried salted fish (Frank and Hess 1941). W. sebi frequently spoils low moisture commodities, particularly sweet (cakes, dates, jams, fruits), salty (fish, meat, peanuts) and or dried (cereal, bread, rice) foods (Samson et al. 2010). This cosmopolitan species is also often isolated from indoor and outdoor air (Takahashi 1997), soil and sea salt (Domsch et al. 2007). Japanese and European researchers first demonstrated that $W$. sebi is abundant in the settled dust of homes (Lustgraaf 1978; Sakamoto et al. 1989; Takahashi 1997) and more recently, it has been frequently detected in house dust in Canada, USA and Western Europe (Amend et al. 2010; Miller and Day 1997; Nonnenmann et al. 2012). This species has been reported to cause both 
cutaneous and sub-cutaneous infections in humans and occupational allergy in farmers (Lappalainen et al. 1998; Reboux et al. 2007). Sensitization to $W$. sebi was first reported in Japan (Sakamoto et al. 1989). This species is known to elicit an IgE response in humans (Simon-Nobbe et al. 2007).

Despite its small genome, $W$. sebi has evolved specific adaptions to thrive in high saline and sugar environments. These adaptations could include altered cell morphology, a compatible solutes strategy, changing plasma membrane fluidity and altered gene expression (GundeCimerman et al. 2009). Increased cell wall thickness and decreased hyphal compartment length have been reported from Wallemia species in response to high salt concentrations (Kuncic et al. 2010). Three gene families found in Wallema are thought responsible for the ability to adapt to conditions with high osmotic tension: HSP20, Dabb and AA_trans genes. Some of these are also found in in poplar (Populus balsamifera) (Padamesee et al. 2012). This intriguing fungus has received increased attention due to its unique phylogenetic position, its ability to at adapt to harsh environments and increased awareness of its health implications.

There are a limited number of reports regarding secondary metabolites from $W$. sebi. This is most likely due to its xerophilic nature that makes it difficult to culture by conventional methods. A strain isolated from a spoiled sweet cake produced the sesquiterpenes walleminol and walleminone (figure 4.2; Frank et al. 1999). These caryophyllenes were previously designated walleminol A and B by Wood et al. (1990) respectively. Walleminol has an $\mathrm{LD}_{50}$ of $40 \mu \mathrm{g} \mathrm{mL} \mathrm{m}^{-1}$ for brine shrimp and a minimum inhibitory dose of $50 \mu \mathrm{g} \mathrm{mL}^{-1}$ for rat liver cells (Wood et al. 1990). The tryptophan metabolite tryptophol was also isolated as a major impurity during the 
purification of walleminol (Wood et al. 1990). Tryptophol is an endogenous plant constituent and growth regulator that is also a byproduct of alcohol fermentation by S. cerevisiae. It has also been reported to have cytotoxic and genotoxic effects in human cell lines (Kosalec et al. 2008). The antibiotic antitumor azasteroids UCA 1064-A and UCA 1064-B (figure 4.2) have also been reported from $W$. sebi. The latter compound demonstrated weak activity against Saccharomyces cerevisea, Gram positive bacteria and was cyctotoxic to HeLa cells ( $\mathrm{IC}_{50}, 15 \mu \mathrm{M}$; Takahashi et al. 1992). A cyclopentanopyridine alkaloid (figure 4.2) with modest activity to Enterobacter aerogenes and weak cytotoxic was reported from a marine halotolerant $W$. sebi isolate along with eleven known aromatic compounds by Peng et al. (2011). This species is also reported to produce the pyrrol-2-ylpolyene pigments wallemia A- F (figure 4.2; Badar et al. 1973; Ito et al. 1981) and hemolytic compounds in response to salt stress (Botic et al. 2012). Kogej et al. (2006) screened various xerophilic fungi for mycosporine production when cultured on a medium of high osmotic tension. No mycosporine production was observed for the one strain of $W$. sebi studied. Figure 4.2 illustrates some previously reported secondary metabolites from W. sebi.

There are no reports of the metabolites produced by strains of $W$. sebi from the USA or Canada. Here, metabolites isolated from $W$. sebi UAMH 7897, a strain recovered from dust in a school vent in northern Saskatchewan, were isolated and identified. Additional indoor strains were shown to produce the same metabolites in similar yields. 


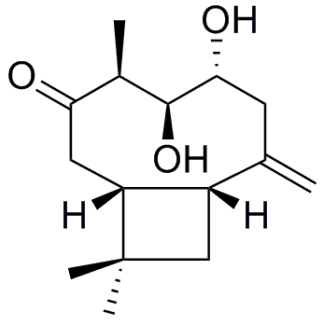

Walleminone

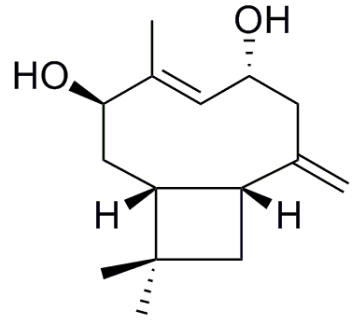

Walleminol<smiles>CC1CC(=O)c2ncc(O)cc21</smiles>

Cyclopentanopyridine alkaloid<smiles>CC(/C=C/C=C/C(O)=C/C(=O)C(C)C)=C\c1ccc[nH]1</smiles>

Wallemia A

Figure 4.2: Secondary metabolites previously reported from $W$. sebi. 


\section{RESULTS AND DISCUSSION}

\section{Isolation and Structural Characterization of $\boldsymbol{W}$. sebi Metabolites}

The W. sebi strains (table 4.4) examined for metabolites produced the compounds isolated in similar amounts (table 4.4). All metabolites isolated and structurally characterized by NMR and mass spectrometry were done with $W$. sebi UAMH 7897 extracts as it was easily grown in liquid culture compared to some of the other strains investigated here. Walleminone (4.1) and tryptophol (4.3), both previously reported from $W$. sebi, a structurally new compound named wallimidione (4.2) here and tryptophol acetate (4.4), phenylacetic acid (4.5) and $p$ hydroxybenzaldehyde (4.6), reported from $W$. sebi for the first time, were isolated from the EtOAc extract of the liquid culture. Their isolation and structural elucidation are described below.

Walleminone (4.1); $4.2 \mathrm{mg}$; colorless oil; $[\alpha]_{\mathrm{D}}^{25}+35$ (c 0.2, MeOH); UV (MeOH)/nm $\lambda_{\max }(\log$ ع) 296 (2.20), 230 (2.56); HRESIMS $m / z 253.1819[\mathrm{M}+\mathrm{H}]^{+}$(calculated for $\mathrm{C}_{15} \mathrm{H}_{25} \mathrm{O}_{3}, 253.1804$ ). ${ }^{1} \mathrm{H}$ NMR $\left(\mathrm{CD}_{3} \mathrm{OD}, 400 \mathrm{MHz}\right)$ and ${ }^{13} \mathrm{C} \mathrm{NMR}\left(\mathrm{CD}_{3} \mathrm{OD}, 100 \mathrm{MHz}\right)$ data were consistent with published data by Frank et al. (1999) and can be found in table 4.1. The structure of walleminone can be found in figure 4.3. 


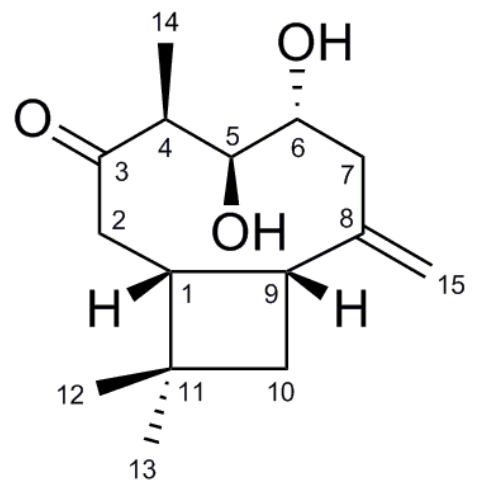

Figure 4.3: Structure of Walleminone (4.1).

Table 4.1: ${ }^{1} \mathrm{H}(400 \mathrm{MHz})$ and ${ }^{13} \mathrm{C}$ NMR Data (100 MHz) Walleminone (4.1) in $\mathrm{CD}_{3} \mathrm{OD}$.

\begin{tabular}{ccc}
\hline Position & $\delta_{\mathrm{C}, \text { type }}$ & $\delta_{\mathrm{H}}(\mathrm{J}, \mathrm{Hz})$ \\
\hline $\mathbf{1}$ & $50.2, \mathrm{CH}$ & $2.29, \mathrm{~m}$ \\
$\mathbf{2}$ & $42.6, \mathrm{CH}_{2}$ & $2.05, \mathrm{dd}(1.8,12.8)$ \\
$\mathbf{3}$ & & $2.88, \mathrm{dd}(10.8,12.8)$ \\
$\mathbf{4}$ & $224.0, \mathrm{C}$ & $3.31, \mathrm{~m}$ \\
$\mathbf{5}$ & $48.2, \mathrm{CH}$ & $3.45, \mathrm{dd}(2.8,10)$ \\
$\mathbf{6}$ & $79.8, \mathrm{CH}$ & $3.34, \mathrm{dd}(2.6,10)$ \\
$\mathbf{7}$ & $71.7, \mathrm{CH}$ & $2.30, \mathrm{~m}$ \\
& 45.2, & $2.42, \mathrm{dd}(11.0,14.6)$ \\
$\mathbf{8}$ & & $3.15, \mathrm{~m}$ \\
$\mathbf{9}$ & $147.8, \mathrm{C}$ & $1.69, \mathrm{ddd}(3.0,8.1,11.2)$ \\
$\mathbf{1 0}$ & $38.2, \mathrm{CH}$ & $2.00, \mathrm{dd}(11.1,11.2)$ \\
$\mathbf{1 1}$ & 36.1 & $1.26, \mathrm{~s}$ \\
$\mathbf{1 2}$ & $34.5, \mathrm{C}$ & $0.90, \mathrm{~s}$ \\
$\mathbf{1 3}$ & $30.1, \mathrm{CH}_{3}$ & $1.11, \mathrm{~d}(7.0)$ \\
$\mathbf{1 4}$ & $25.1, \mathrm{CH}_{3}$ & $5.09, \mathrm{~d}(1.5)$ \\
& 14.0 & $5.32, \mathrm{bs}$ \\
\hline
\end{tabular}

1-Benzylhexahydroimidazo [1,5- $\alpha$ ] pyridine-3,5-dione (4.2) was isolated as a light yellow oil with the molecular formula $\mathrm{C}_{14} \mathrm{H}_{16} \mathrm{~N}_{2} \mathrm{O}_{2}$ determined by HRESIMS with $m / z 245.1293[\mathrm{M}+\mathrm{H}]^{+}$ (calculated for 245.1290). The UV spectrum displayed absorption maxima at 275, 248 and 220 $\mathrm{nm}$, indicating the presence of a conjugated system within the molecule. Examination of the ${ }^{1} \mathrm{H}$ 
and ${ }^{13} \mathrm{C}$ NMR data (Table 4.2), displayed three $\mathrm{sp}^{2}$ quaternary carbons including two carbonyls and one aromatic carbon, five aromatic methines, two $\mathrm{sp}^{3}$ methines and four $\mathrm{sp}^{3}$ methylenes.

Analysis of ${ }^{1} \mathrm{H}$ NMR spectroscopic data revealed the presence of a mono-substituted aromatic ring. In the HMBC spectrum, the cross-peak observed between the methylene protons at $\delta 3.16$ $(\mathrm{dd}, J=2.7,5.1 \mathrm{~Hz})$ and the aromatic carbons at $\delta 137.3, \delta 129.4$ and $\delta 131.0$, located this methylene group at the benzylic position.

In the COSY, an extended spin system was observed from the benzylic methylene protons at $\delta$ 3.16, sequentially to the two aliphatic methines at $\delta 4.44(\mathrm{~m})$ and $\delta 4.04$ (ddd, $J=1.7,6.710 .9$ $\mathrm{Hz})$, to the three methylenes at $\delta 1.75-1.83(\mathrm{~m}), \delta 2.10,1.19(\mathrm{~m})$ and $\delta 3.37(\mathrm{~m}), 3.53(\mathrm{dt}, J=8.0$, 12.0 Hz). The terminal unequivalent methylene protons, $\mathrm{H}-6$, in the spin system showed HMBC correlations with a carbonyl at $\delta 170.0$. The two methines at $\delta 4.44$ and $\delta 4.04$ displayed HMBC correlations with carbonyls at $\delta 170.0$ and $\delta 166.9$ respectively. The carbon chemical shifts of the methines at $\delta 57.7$ and $\delta 60.0$ suggested that they are nitrogenated. It was deduced that the two nitrogens directly bound to the methines form amide bonds with the respective carbonyls at $\delta$ 170.0 and $\delta$ 166.9. The high resolution mass and spectroscopic data indicated the presence of an imidazo $[1,5-\alpha]$ pyridine-3,5-dione substituted with a benzyl moiety.

The structure of (4.2) was determined to be 1-benzylhexahydroimidazo [1,5 $]$ pyridine-3, 5dione, given the common name wallimidione here. The relative stereochemistry of positions H-1 and H-8a were determined by NOE. An NOE correlation was observed between $\mathrm{H}-1 / \mathrm{H}-8 \mathrm{a}$ indicating that the two stereogenic methines are in the same plane. 


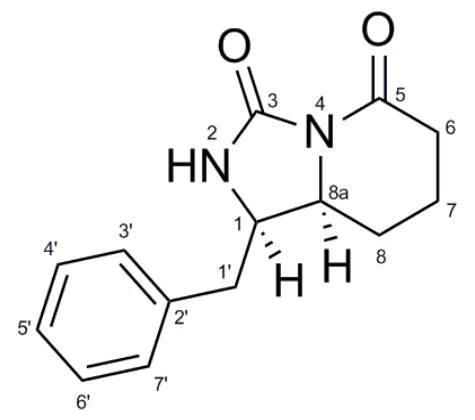

Figure 4.4: Structure of Wallimidione (4.2).

Wallimidione (4.2); $3.7 \mathrm{mg}$; light yellow oil; $[\alpha]^{25}$ - 68 (c 0.2, MeOH); UV (MeOH)/nm $\lambda_{\max }$ $(\log \varepsilon) 275$ (2.72), 248 (2.99) 220 (3.11); HRESIMS m/z 245.1293 [M+H] $]^{+}$(calculated for $\left.\mathrm{C}_{14} \mathrm{H}_{17} \mathrm{~N}_{2} \mathrm{O}_{2}, 245.1290\right) ;{ }^{1} \mathrm{H}$ NMR $\left(\mathrm{CD}_{3} \mathrm{OD}, 400 \mathrm{MHz}\right)$ and ${ }^{13} \mathrm{C} \mathrm{NMR}\left(\mathrm{CD}_{3} \mathrm{OD}, 100 \mathrm{MHz}\right)$ data are reported in table 2. The structure of wallimidione can be found in figure 4.4.

Table 4.2: ${ }^{1} \mathrm{H}(400 \mathrm{MHz})$ and ${ }^{13} \mathrm{C}$ NMR Data (100 MHz) Wallimidione (4.2) in $\mathrm{CD}_{3} \mathrm{OD}$.

\begin{tabular}{ccc}
\hline Position & $\delta_{\mathrm{C}, \text { type }}$ & $\delta_{\mathrm{H}}(J, \mathrm{~Hz})$ \\
\hline $\mathbf{1}$ & $57.7, \mathrm{CH}$ & $4.44, \mathrm{~m}$ \\
$\mathbf{3}$ & $166.9, \mathrm{C}$ & \\
$\mathbf{5}$ & $170.0, \mathrm{C}$ & $3.37 \mathrm{~m}$ \\
$\mathbf{6}$ & $45.9, \mathrm{CH}_{2}$ & $3.53 \mathrm{dt}(8.3,12.0)$ \\
& & $1.75-1.83 \mathrm{~m}$ \\
$\mathbf{7}$ & $22.7, \mathrm{CH}_{2}$ & $2.10 \mathrm{~m}$ \\
$\mathbf{8}$ & $29.4, \mathrm{CH}_{2}$ & $1.19 \mathrm{~m}$ \\
& & $4.04 \mathrm{ddd}(1.7,6.5,10.9)$ \\
$\mathbf{8 a}$ & $60.0, \mathrm{CH}$ & $3.16 \mathrm{dd}(2.7,5.1)$ \\
$\mathbf{1}$ & $38.2, \mathrm{CH} 2$ & $7.20-7.30$ \\
$\mathbf{2}$ & $137.3, \mathrm{C}$ & $7.20-7.30$ \\
$\mathbf{3}$ & $131.0, \mathrm{CH}$ & $7.20-7.30$ \\
$\mathbf{4}$ & $129.4, \mathrm{CH}$ & $7.20-7.30$ \\
$\mathbf{5}$ & $128.1, \mathrm{CH}$ & $7.20-7.30$ \\
$\mathbf{6}$ & $129.4, \mathrm{CH}$ & \\
$\mathbf{7}$ & $131.0, \mathrm{CH}$ & \\
\hline
\end{tabular}




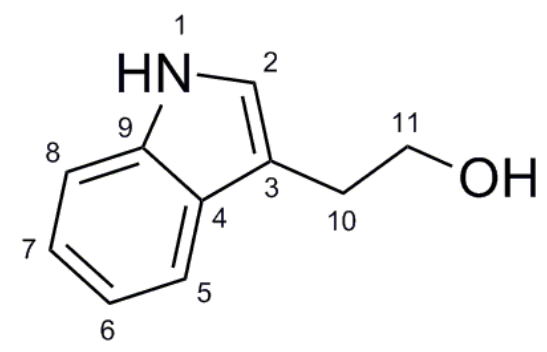

Figure 4.5: Structure of Tryptophol (4.3).

Tryptophol (4.3); $16.3 \mathrm{mg}$; brown solid; ${ }^{1} \mathrm{H}$ NMR $\left(\mathrm{CD}_{3} \mathrm{CN}, 400 \mathrm{MHz}\right) \delta 9.10$ (bs, $\left.1 \mathrm{H}, \mathrm{H}-1\right), 7.58$ (d, 1H, H-8), 7.39 (d, 1H, H-5), 7.10-7.14 (m, 1H, H-6), 7.09 (s, 1H, H-2), 7.02-7.06 (m, 1H, H7), 3.76 (t, 2H, H-10), 2.93 (t, 2H, H-11); ${ }^{13} \mathrm{C}$ NMR (CD3CN, $\left.100 \mathrm{MHz}\right) \delta 137.8$ (C-4), 128.5 (C9), 123.6 (C-2), 122.2 (C-6), 119.5 (C-8), 119.4 (C-7), 113.1 (C-5), 112.0 (C-3), 62.9 (C-11), $29.3(\mathrm{C}-10)$; LRESIMS $m / z$ 162.02 $[\mathrm{M}+\mathrm{H}]^{+}$(calculated for $\left.\mathrm{C}_{10} \mathrm{H}_{12} \mathrm{NO}, 162.0919\right)$. The structure of tryptophol (4.3) can be found in figure 4.5. The presence of tryptophol in the crude filtrate extract was confirmed by an analytical standard (Sigma).

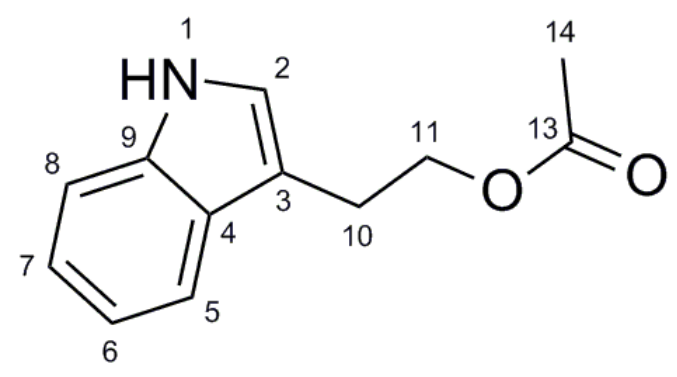

Figure 4.6: Structure of Tryptophol acetate (4.4). 
Tryptophol acetate (4.4); $49.2 \mathrm{mg}$; brown-red oil; ${ }^{1} \mathrm{H}$ NMR $\left(\mathrm{CD}_{3} \mathrm{CN}, 400 \mathrm{MHz}\right) \delta 9.13$ (bs, $1 \mathrm{H}$, H-1), 7.58 (d, 1H, H-8), 7.38 (d, 1H, H-5), 7.12 (m, 1H, H-6), 7.03 (m, 1H, H-7), 4.28 (t, 2H, H11), 3.04 (t, 2H, H-10), 1.93 (s, 3H, H-14); ${ }^{13} \mathrm{C}$ NMR (CD3CN, $\left.100 \mathrm{MHz}\right) \delta 171.8$ (C-13), 137.4 (C-4), 128.3 (C-9), 124.1 (C-2), 122.6 (C-6), 119.8 (C-8), 119.4 (C-7), 112.3 (C-5), 65.2 (C-11), 25.2 (C-10), 21.2 (C-14); LRESIMS m/z $204.12[\mathrm{M}+\mathrm{H}]^{+}$(calculated for $\mathrm{C}_{12} \mathrm{H}_{14} \mathrm{NO}_{2}, 204.1024$ ). The structure of tryptophol acetate (4.4) can be found in figure 4.6.

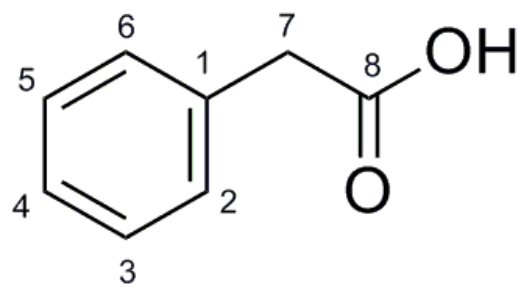

Figure 4.7: Structure of Phenylacetic acid (4.5).

Phenylacetic acid (4.5); $12.9 \mathrm{mg}$; clear solid; ${ }^{1} \mathrm{H}$ NMR $\left(\mathrm{CD}_{3} \mathrm{CN}, 400 \mathrm{MHz}\right) \delta 7.91$ (bs, $1 \mathrm{H}, \mathrm{OH}-$ 9), 7.25-7.36 (m, 5H, H-2 to 6), 3.62 (s, $2 \mathrm{H}, \mathrm{H} 7) ;{ }^{13} \mathrm{C} \mathrm{NMR}\left(\mathrm{CD}_{3} \mathrm{OD}, 100 \mathrm{MHz}\right) \delta 173.6(\mathrm{C}-8)$, 135.7 (C-1), 130.4 (C-2), 130.4 (C-6), 129.4 (C-3), 129.4 (C-5), 127.8 (C-4), 41. (C-7); LRESIMS $m / z 134.9[\mathrm{M}-\mathrm{H}]^{-}$(calculated for $\mathrm{C}_{8} \mathrm{H}_{7} \mathrm{O}_{2}, 135.0446$ ). The structure of phenyl acetic acid (4.5) can be found in figure 4.7. The presence of phenylacetic acid in the crude filtrate extract was confirmed by an analytical standard (Sigma). 


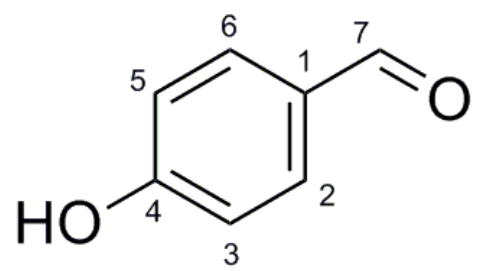

Figure 4.8: Structure of $p$-Hydroxybenzaldehyde (4.6).

p-Hydroxybenzaldehyde (4.6); $17.2 \mathrm{mg}$; light yellow oil; ${ }^{1} \mathrm{H}$ NMR $\left(\mathrm{CD}_{3} \mathrm{CN}, 400 \mathrm{MHz}\right) \delta 9.80$ (bs, 1H, H-7), 7.72 (d, 2H, H-2 and H-6), 6.91 (d, 2H, H-3 and H-5); ${ }^{13} \mathrm{C}$ NMR $\left(\mathrm{CD}_{3} \mathrm{OD}, 100\right.$ MHz) $\delta 191.2$ (C-7), 162.1 (C-4), C-130.4 (C-1), 132.3 (C-2, C-6), 115.8 (C-3, C-5); LRESIMS $m / z 120.9$ [M-H] $^{-}$(calculated for $\mathrm{C}_{7} \mathrm{H}_{5} \mathrm{O}_{2}, 121.0290$ ). The structure of $p$-hydroxybenzaldehyde (4.6) can be found in figure 4.8 .

From a representative strain (UAMH 7897), walleminone (4.1), wallimidione (4.2), tryptophol (4.3), tryptophol acetate (4.4), phenylacetic acid (4.5) and p-hydroxybenzaldehyde (4.6) were isolated and characterized by NMR spectroscopy. The proton and carbon spectra for walleminone isolated here matched the reported data of Frank et al. (1999). We did not isolate or observe walleminol by LC-UV-MS however this is not surprising. Natural interconversion of walleminol to walleminone most likely occurs by an epoxidation of the $E$-alkene in walleminol followed by a subsequent rearrangement to walleminone. This is not unprecedented for other naturally occurring caryophyllenes (Frank et al. 1999).

The structure wallimidione (4.2) appears to be rather unusual. No other natural products with the same hexahydroimidazo $[1,5 \alpha]$ pyridine-3,5-dione core structure could be found in a substructure similarity search of the ACS database SciFinder ${ }^{\circledR}$. An analysis for structural alerts of 
walleminone of with TOXTREE (EU Joint Research Centre, Institute for Health and Consumer Protection) confirmed the available data showing modest toxicity. However, a similar analysis of the novel compound wallimidione revealed several indications of toxicity, requiring further in vitro testing as was done with the previously discussed Chaetomium and Penicillium metabolites. However, due to sample limitation and additional toxicity assays aimed at determining the role of fungal secondary metabolites impact on human health, this was not performed.

The identification of the tryptophan metabolite tryptophol was not surprising as it was reported as a major impurity during the isolation of walleminol A (Wood et al. 1990). The presence of tryptophol was confirmed by an analytical standard. The related compound, tryptophol acetate was additionally isolated and this appears to be a first report from this species. This compound has been previously isolated from the fungus Ceratocystis fagacearum, a pathogen of the oak tree (Fenn et al. 1977). This also appears to be the first report of phenylacetic acid and $p$ hydroxybenzaldehyde from $W$. sebi of which the former metabolite was confirmed by an analytical standard. Phenylacetic acid has been reported from numerous fungi including Penicillium herquei (Ding et al. 2008), Penicillium digitatum (Ariza et al. 2002), as well as Biscogniauxia mediterranea (Evidente et al. 2005). p-hydroxybenzaldehyde has been reported from Ophiostoma crassivaginata and an unidentified endophyte of a Chilean coniferous tree, Prumnopitys andina (Schmeda-Hirschmann et al. 2005).

Structurally similar para-substituted aromatic metabolites and other indole compounds were reported by Peng et al. (2011) who additionally characterized a characterized a new cyclopentanopyridine alkaloid from $W$. sebi. We did not isolate or observe any pyrrol pigments, 
wallemia A- F (Badar et al. 1973), or nitrogen bearing sterol similar to UCA1064-A and B (Takahashi et al. 1993). The metabolite data here would somewhat agree with the observations of Kogej et al. (2006), that this fungus produces metabolites that are not UV active. All strains examined here were fermented in triplicate using Roux bottles and screened by LC-UV-MS. All W. sebi strains investigated produced the same metabolites in similar yields (table 4.4).

Table 4.3: Metabolite production by indoor W. sebi strains examined.

\begin{tabular}{|c|c|c|c|c|c|c|}
\hline & \multicolumn{6}{|c|}{ Compound } \\
\hline Strain & $\mathbf{4 . 1}$ & $\mathbf{4 . 2}$ & $\mathbf{4 . 3}$ & $\mathbf{4 . 4}$ & $\mathbf{4 . 5}$ & $\mathbf{4 . 6}$ \\
\hline UAMH 7897 & + & + & +++ & +++ & +++ & ++ \\
\hline CBS 463.97 & + & + & + & ++ & ++ & + \\
\hline DAOM 226641 & + & + & +++ & +++ & +++ & ++ \\
\hline DAOM 226642 & + & + & ++ & +++ & +++ & + \\
\hline DAOM 242570 & + & + & +++ & +++ & +++ & ++ \\
\hline DAOM 242571 & + & + & ++ & +++ & +++ & ++ \\
\hline
\end{tabular}

$+<1 \mathrm{mg} \mathrm{L}^{-1}$

$++>1 \mathrm{mg} \mathrm{L}^{-1},<10 \mathrm{mg} \mathrm{L}^{-1}$

$+++>10 \mathrm{mg} \mathrm{L}^{-1}$

Until recently, there was little information concerning the fungi that naturally inhabit hypersaline environments. Most studies have focused on bacteria and Archaea as it was believed that eukaryotic organisms could not adapt to these extreme environments. However, extremophilic fungi are now known to be found around the planet where tolerance to low water activity has been observed within 10 of the 140 orders of known fungi (Sepcic et al. 2011). The fungi 
identified as xerophilic were not closely related. Tolerance to low $\mathrm{a}_{\mathrm{w}}$ was typically limited to a few species within each order. This indicates multiple evolutionary adaptations have been made by phylogenetically distinct xerophilic fungi to deal with high osmotic tension. Adaptations of xerophilic fungi include altered gene expression in response to solute concentrations, the accumulation of sugar alcohols in the cytoplasm, changing plasma membrane fluidity and perhaps secondary metabolite production (Gunde-Cimerman et al. 2009; Petterson and Leong 2011).

The majority of food-borne fungi synthesize secondary metabolites when the water activity of their substrate is high, close to 1.0 and at mesophilic temperatures $\left(20-25^{\circ} \mathrm{C}\right)$ (Frisvad and Thrane 1995). Despite the obvious physical differences between cold temperatures and high solute commodities, the common stressor for fungal growth is low water activity. There are only a few exceptions where secondary metabolite production is stimulated by typically unfavorable conditions such as low temperatures, desiccation or high solute concentrations that generate low water activity environments. This was observed with $W$. sebi as walleminone was produced in higher quantities as the amount of solute added to the liquid media increased lowering the water activity (Wood et al. 1990; Frank et al. 1999). In the present study, similar observations were made. The liquid media used here was the same as reported by Frank et al. (1999). Kogej et al. (2006) reported increased mycosporine production with increased osmotic tension in Cladosporium sphaerospermum, Cryptococcus liquefaciens, and Aureobasidium pullans. However, this was not observed for the one $W$. sebi isolate studied nor were these UV absorbing compounds observed in the extracts examined in this study. Botic et al. (2011) suggested unsaturated fatty acids induced by high salt concentrations lyse mammalian erythrocytes in $W$. sebi. Sepcic et al. (2011) examined the antimicrobial activity of crude extracts from xerophilic 
fungi under conditions for mesophilic growth and low $\mathrm{a}_{\mathrm{w}}$ (low temperature, increased glucose and salt concentrations). Increased activity was observed in the extracts of xerophilic, stressed fungi. This suggested that low water activity conditions may induce the production of bioactive secondary metabolites from xerophilic fungi. Aqueous extracts were not active relative to organic extracts further suggesting the antimicrobial effects are due to secondary metabolites. 


\section{EXPERIMENTAL}

\section{W. sebi Cultures}

The six $W$. sebi strains examined here for metabolites are deposited in culture collections and were isolated from indoor environments in Canada and the Netherlands, see table 4.5. Cultures were aseptically transferred to sterile $2 \%$ yeast extract (Difco), $20 \%$ sucrose agar plates and slants. These were incubated at $25^{\circ} \mathrm{C}$ until sufficient growth was observed. After this incubation period, plates and slants were sealed with Parafilm and stored at $4^{\circ} \mathrm{C}$ until required.

Table 4.4: W. sebi strains examined for metabolite production and fungal source.

\begin{tabular}{lll}
\hline Strain & Location & Sampling Material \\
\hline UAMH 7897 & Saskatchewan, Canada & Indoor dust \\
CBS 463.97 & South Holland, Netherlands & Indoor dust \\
DAOM 226641 & Ottawa, Canada & Indoor dust \\
DAOM 226642 & Ottawa, Canada & Indoor dust \\
DAOM 242570 & Ottawa, Canada & Indoor dust \\
DAOM 242571 & Ottawa, Canada & Indoor dust \\
\hline
\end{tabular}

\section{Fermentation and Metabolite Screening}

A slant of each $W$. sebi described in table 4.4 was macerated with a Polytron homogenizer in sterile distilled deionized water and a 5\% (v/v) aliquot was utilized to inoculate Roux bottles in triplicate containing $200 \mathrm{~mL}$ of autoclaved $2 \%$ yeast extract media supplemented with $20 \%$ sucrose. Each Roux bottle was incubated stationary at $25^{\circ} \mathrm{C}$ in the dark for 6 weeks in a growth chamber. 
The liquid culture was separated from the cells after the six week incubation period by vacuum filtration through a Whatman \#4 filter paper. The cells were frozen, lyophilized and stored in the freezer at $-20^{\circ} \mathrm{C}$, and the culture filtrate volume and $\mathrm{pH}$ were recorded. The culture filtrates were saturated with $\mathrm{NaCl}$, exhaustively extracted with ethyl acetate (EtOAc), passed through a Whatman \# 1 filter paper and anhydrous sodium sulfate prior to being dried by rotary evaporation. The dry crude extract was re-dissolved in a minimal amount of HPLC grade methanol, filtered through a $25 \mathrm{~mm}$ PTFE $(0.2 \mu \mathrm{m})$ syringe filter, and dried under a gentle stream of nitrogen gas.

Each dry $W$. sebi filtrate extract was dissolved in $2 \mathrm{~mL}$ of HPLC grade methanol and analyzed by LC-UV-MS. This was performed using a Waters 2795 separations module, Waters 996 diode array detector, and Micromass Quatro LC mass spectrometer operated in both positive and negative mode. Compounds were separated by a Phenomenex Gemini $C_{18}(150 \times 4.60 \mathrm{~mm}, 3$ $\mu \mathrm{m}, 110 \AA$ ) column (Torrance, California) using a mobile phase consisting of $\mathrm{ACN}-\mathrm{H}_{2} \mathrm{O}$ with FA; [0.1\%, (v:v)]. The solvent gradient was linear programmed from 5 to $100 \%$ ACN over 13 minutes with a flow rate of $1.0 \mathrm{~mL} \mathrm{~min}^{-1}$. Based on the LC-UV-MS data, $W$. sebi UAMH 7897 was selected as a representative strain as the chromatograms from each strain were similar.

Calibration plots for compounds (4.1-4.6) were constructed using identical LC conditions described above for screening extracts when the MS was operated in SIM mode. The $[\mathrm{M}+\mathrm{H}]^{+}$ion was utilized for 4.1-4.4 whereas the $[\mathrm{M}-\mathrm{H}]^{-}$was used for 4.5 and 4.6. Each standard solution and extract was analyzed in both positive and negative mode. Each standard solution was analyzed in triplicate and the calibration plot was represented by $0.01,0.1,1.0$, and $10 \mu \mathrm{g}$ purified compound 
on column. However, for compounds 4.1 and 4.2, a $10 \mu \mathrm{g}$ standard was not analyzed due to sample limitation. The approximate concentrations are reported as the mean of triplicate fermentations of each strain in Roux bottles. Besides NMR data, the presence of both tryptophol (4.3) and phenylacetic acid (4.5) were confirmed by an authentic standard (Sigma).

\section{Large Scale Fermenation and Metabolite Isolation}

A slant of $W$. sebi UAMH 7897 was macerated in sterile distilled deionized water and a 5\% (v/v) aliquot was utilized to inoculate twenty $250 \mathrm{~mL}$ Erlenmeyer flasks containing $50 \mathrm{~mL}$ of $2 \%$ yeast extract, $20 \%$ sucrose sterile liquid media. After one week, individual starter cultures were macerated and aseptically transferred to Glaxo bottles containing $1 \mathrm{~L}$ of the same media. Cultures were fermented and extracted as described above.

The crude extract (4.6 g) was initially dissolved in a minimal amount of $\mathrm{CHCl}_{3}$ with a couple drops of $\mathrm{MeOH}$ and fractionated by normal phase column chromatography. The extract was applied to a silica gel column $(40-63 \mu \mathrm{m})$ and metabolites were separated using a $\mathrm{MeOH}-\mathrm{CHCl}_{3}$ gradient increasing from 0 to $10 \% \mathrm{MeOH}$ in $1 \%$ increments followed by flushing with 20 and $50 \% \mathrm{MeOH}_{\text {in }} \mathrm{CHCl}_{3}$. Column fractions were screened by TLC $\left(0.2 \mathrm{~mm}\right.$ silica gel $60 \mathrm{~F}_{254}$ precoated) using $10 \%(\mathrm{v} / \mathrm{v}) \mathrm{MeOH}-\mathrm{CHCl}_{3}$ and visualized under both long wave $(365 \mathrm{~nm})$ and short wave $(254 \mathrm{~nm})$ light. Like fractions were combined, dried by rotary evaporation and additionally screened by LC-UV-MS as described for crude $W$. sebi filtrate extracts. This yielded eleven fractions that were subjected to semi-preparative reverse phase HPLC. The linear gradient was programmed from $10-80 \% \mathrm{ACN}-\mathrm{H}_{2} \mathrm{O}$ over 19 minutes with a flow rate of $4 \mathrm{ml} \mathrm{min} \mathrm{m}^{-1}$ and a 
Phenomenex Luna $\mathrm{C}_{18}(250 \times 10.00 \mathrm{~mm}, 5 \mu \mathrm{m}, 100 \AA)$ column was utilized. Fraction 1 eluted in $1 \% \mathrm{MeOH}$ yielding $4.1(4.2 \mathrm{mg})$ and $4.2(3.7 \mathrm{mg})$, fraction 2 eluted in $2 \% \mathrm{MeOH}$ yielding 4.4 (49.2 mg), fraction 4 eluted in 3\% MeOH yielding $4.3(16.3 \mathrm{mg})$, fraction 7 eluted in $5 \% \mathrm{MeOH}$ yielding $4.6(17.2 \mathrm{mg})$, and fraction 8 eluted in $6 \% \mathrm{MeOH}$ yielding $4.5(12.9 \mathrm{mg})$.

\section{General Experimental Procedures}

Metabolite NMR spectra were obtained on a Bruker Avance 400 Spectrometer (Milton, On) at $400.13\left({ }^{1} \mathrm{H}\right)$ and $100 \mathrm{MHz}\left({ }^{13} \mathrm{C}\right)$ using a $5 \mathrm{~mm}$ auto-tuning broadband probe with a Z-gradient. Secondary metabolites were dissolved in $\mathrm{CD}_{3} \mathrm{CN}(\delta \mathrm{H} 1.94$ and $\delta \mathrm{C} 118.7 / 1.39)$ or $\mathrm{CD}_{3} \mathrm{OD}(\delta \mathrm{H}$ 3.31 or $\delta \mathrm{C} 49.1$ ) (CDN Isotopes, Point Claire, QC) and were referenced to the solvent peak.

Chemicals shifts were assigned based on ${ }^{1} \mathrm{H},{ }^{13} \mathrm{C}, \mathrm{COSY}$, HSQC, HMBC, and DEPT spectra utilizing standard Bruker pulse sequences. HR-MS data were acquired on a system equipped with an Agilent 1100 binary pump connected to an Agilent G1969A LC/MSD TOF HRMS. Compounds were separated on a Phenomenex Zorbax $\mathrm{C}_{18}(50 \times 3.0 \mathrm{~mm}, 1.8 \mu \mathrm{m})$ column using a mobile phase consisting of ACN- $\mathrm{H}_{2} \mathrm{O}$ with FA; $[0.1 \%$, (v:v)]. The gradient was linear programmed from 10 to $95 \% \mathrm{ACN}$ over 4 minutes at a flow rate of $1 \mathrm{~mL} \mathrm{~min}^{-1}$. Optical rotations were measured using an Autopol IV polarimeter (Rudolph Analytical, NJ). UV spectra were obtained on a Varian Cary 3 UV-visible spectrophotometer scanning from 800-190 nm. 


\section{CHAPTER V}

ISOLATION AND STRUCTURAL ELUCIDATION OF SECONDARY METABOLITES

FROM TRICHODERMA CITRINOVIRIDE, T. KONINGIOPSIS AND T. ATROVIRIDE 


\section{INTRODUCTION}

Species within the genus Trichoderma are very common in nature and frequently found in soil, on decaying organic matter, roots and organic waste. This is a reflection of the ability of these fungi to grow on many diverse substrates including cellulose. The genus is ubiquitous in soil but certain species are also known plant symbionts, fungal pathogens and opportunistic endophytes (Harman et al. 2004). However, the taxonomy of this ecologically important genus is rather convoluted as many species have similar morphology. Thus, many of the gene sequences in NCBI are associated with incorrect identifications. Since the development of molecular methods, the number of Trichoderma species has increased from under 10 to over 100 (Druzhinina et al. 2006). This is exemplified by the most common Trichoderma species in the literature, $T$. viride. The conidia of this species have rough walls; however, the conidia of most Trichoderma species are smooth. In the past, any isolate with green ascospores would be identified as T. viride regardless of the conidia texture (Jaklitsch et al. 2006). Additionally, anamorphic Trichoderma species are intimately related to a teleomorphic Hypocrea (Samuels 1996). The anamorphic Trichoderma state has been principally studied since the teleomorphic Hypocrea state is rarely observed in culture (Chaverri and Samuels 2003). To clarify the taxonomy and determine the ecological role of both stages, the holomorph, both the anamorphic and teleomorphic stages, should be studied. To demonstrate the difficulties associated with Trichoderma taxonomy, it is estimated that approximately $40 \%$ of the Hypocrea and Trichoderma records in GenBank are unidentified isolates or misidentified at the species level (Druzhinina et al. 2006). This is in part due to the lack of quality assurance concerning the species identifications of deposited sequences. Interpretation of reports concerning the ecology, enzyme production, bio-control, and 
secondary metabolites of the genus Trichoderma can be problematic due to the inherent difficulties with their identifications at the species level.

Trichoderma species can be easily identified from other fungal genera by masses of conidia that are typically green but may be colorless or yellow in rare cases. Ascospore pigmentation has been utilized as a means of characterizing genera within Hypocreales (Samuels 1996). Chaverri and Samuels (2003) examined 40 species of Hypocrea and Trichoderma with green ascospores demonstrating that they are derived from within Hypocrea but do not form a monophylogenetic group. The Hypocrea schweintzzi complex that coincides with the anamorphic Trichoderma section Longibrachiatum, including T. citrinoviride, is an example of a monophyletic group where all species have colorless ascospores. There are many issues with Trichoderma taxonomy making it a difficult topic. The number of recognized species is expected to increase because many newly accepted species have been found in poorly sampled areas such as Siberia or South East Asia (Druzhinina et al. 2006). Since most recognized Trichoderma species were identified with the use of molecular methods, their gene sequences are well documented (Chaverri and Samuels 2003). Continued classical analysis of phenotypes of both anamorphs and teleomorphs in combination with molecular methods and metabolite profiles will contribute to the understanding as well as clarifying the difficult taxonomy associated with this genus.

It is believed that so called green Trichderma species thrive because of their efficient production of extracellular cellulases (Flannigan and Miller 2011) and synthesis of a wide variety of bioactive secondary metabolites (Sivithamparam and Ghisalberti 1998). These species have been extensively studied for agricultural bio-control applications as a number of species are plant 
symbionts and demonstrate antifungal or plant growth stimulating activities. Trichoderma species represent a large majority of the bio-control fungi in commerce (Fonzi and Sypherd 1986). They may also be mycoparasites of other fungi. T. longibrachiatum is a known opportunistic pathogen of immunocompromised mammals including humans (Kredics et al. 2011). These competitive species are capable of directly competing for resources and space within a niche, modifying environmental conditions or promoting plant defense mechanisms or growth (Gloer 2007; Harman et al. 2004).

Trichoderma species are also commonly reported in buildings with water damage. As tertiary colonizers, Trichoderma species require the water activity of the substrate to be above 0.90 (Flannigan and Miller 2011). Trichoderma species are primarily associated with different types of damaged wood and plywood (Andersen et al. 2011). Species that have been identified in damp buildings include T. longibrachiatum, T. citrinoviride T. harzianum, T. atroviride, T. viride, and T. harmatum (Lubeck et al. 2000). Lubeck et al. (2000) reported that T. longibrachiatum and its close relative $T$. citrinoviride as the most frequently identified Trichoderma reported indoors in Danish buildings. Working from samples across Scandinavia, Andersen et al. (2011) reported $T$. harzianum as the most common. In the United States and Canada, T. harzianum, T. hamatum, T. koningii and T. viride are common on wood and manufactured wood (Miller et al. 2008). T. viride and T. harzianum were common on wet urea formaldehyde foam insulation in Canada (Bisset 1987) and wet sprayed on cellulose insulation in the US (Godish and Godish 2006). Some of these have been reported to cause infections in immune compromised patients, albeit rarely (Kredics et al. 2011). 
All of the above Trichoderma species are capable of producing a wide variety of structurally diverse secondary metabolites. Some strains used for biological control applications produce simple trichothecenes such trichodermin and harzianum A. These two cytotoxic metabolites were originally reported from $T$. viride (Godtfredsen and Vangedal 1964) and T. harzianum (Corley et al. 1994). However, the strains where trichodermin and harzianum A were originally isolated from were demonstrated to be in fact T. breviocompactum based on metabolite profiles, morphology and ITS sequences (Nielsen et al. 2005). All T. breviocompactum strains investigated were unambiguously shown to produce trichodermin and or harzianum A. Isolates of T. viride, T. harzianum, T. longibrachiatum and T. citrinoviride, where at least one isolate from each species was derived from indoor sources, did not produce either trichothecene. Trichothecene biosynthetic genes have been identified and characterized from $T$. brevicompactum and T. arundinaceum. They were shown to be organized differently than in Fusarium species (Cordoza et al. 2011). Various reports however indicate that trichothecene production by this genus is confined to a few species (Lubeck et al. 2000; Nielsen et al. 2005).

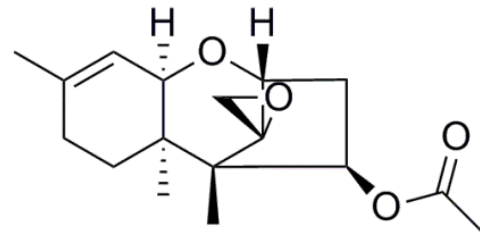

Trichodermin

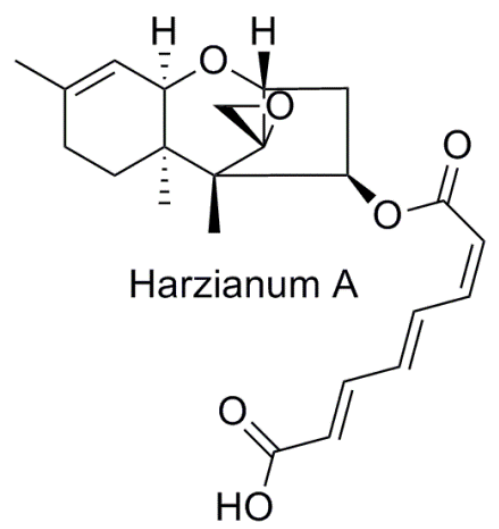

Figure 5.1: Structures of the trichothecenes trichodermin and harzianum A. 
The sulfur containing mycotoxin gliotoxin has been isolated from T. viride (Brian and Hemming 1945) and T. harmantum (Hussain et al. 1975). The steroidal antibiotic viridin has been reported from many Trichoderma species including T. koningii, T. viride and T. virens. However, the producing strains for these two metabolites have more recently been demonstrated to be $T$. virens, which has not been reported indoors (Nielsen 2003; Samson et al. 2000).

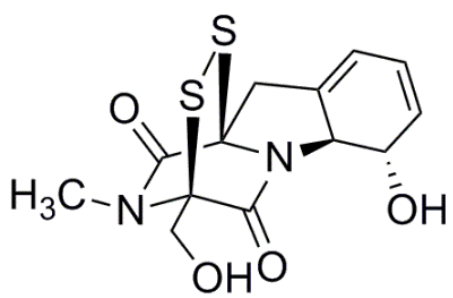

Gliotoxin

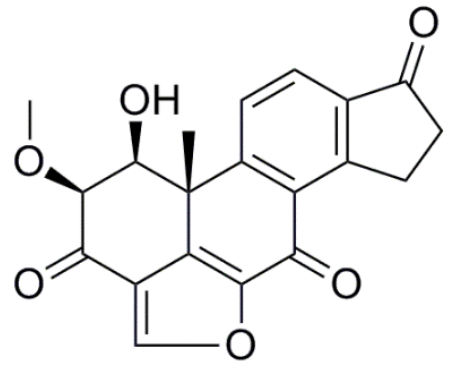

Viridin

Figure 5.2: Structures of gliotoxin and viridin.

Unstable but toxic isocyanide metabolites have been obtained from the fermentation broth of $T$. harmatum, T. koningii and T. harzianum which are proposed biodegradation products of the aromatic amino acid tyrosine (Taylor 1986). Trichoderma isolates have been shown to produce 10-20 unstable isocyandes; however, their isolation is very difficult (Boyd et al. 1991). Isocyanide purification from culture broth can be improved by generating a more stable methyl ester derivative (Brewer et al. 1979) or rhodium pentamethylcyclopentadiene isothiocynate coordination complex (Hanson et al. 1985). Trichoviridin was originally characterized from $T$. koningii and was later isolated with two new isocyandide methyl ester derivatives from $T$. hamatum (Brewer et al. 1979; Tamura et al. 1975). Isonitrins A-D were also isolated from a soil isolated of T. hamatum (Fujiwara et al. 1982). Unlike most fungal metabolites, isocynides are 
volatile (Taylor 1986). Isocyanides produced by T. hamatum have been implicated in ovine illthrift by disrupting rumen bacteria (Brewer et al. 1982).

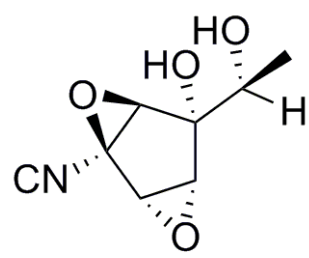

Trichoviridin

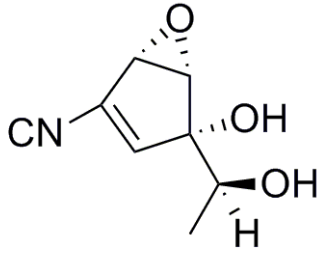

Isonitrin B

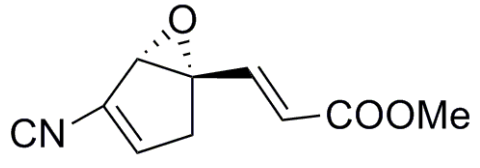

Isonitrile Methyl Ester

Figure 5.3: Structures of trichoviridine, isonitrin B, and a isocyanide isolated as a its methyl ester.

T. harzianum produces a variety of alpha pyrones and polyketide structures similar to the koninginins produced by T. koningii (Ghisalberti and Rowland 1993). T. koningii is one of the most commonly reported Trichoderma species in the literature and was recently phylogenetically investigated (Samuels et al. 2006). Combining molecular data and phenotypic characters, three distinct evolutionary lineages were revealed. T. koningiopsis belongs to lineage 1 with $T$. koningii. This species is known to produce a variety of koninginins that exhibit modest bioactivities (Ghisalberti and Rowland 1993). Almost all of these Trichoderma metabolites discussed have been from species that are categorized as so called green Trichoderma species. $T$. longibrachiatum, a yellow Trichoderma species, produces trichodimerol, bisvertinol, bisvertinolone and trichodermolide derived from a common precursor, sorbicillin (Andrade et al. $1992 ; 1996)$.

Besides synthesizing a wide variety of small molecules, some species of Trichoderma are currently the richest source of modified, bioactive, non-ribosomal peptides called peptaibols 
(Leitgeb et al. 2007). Since they result from a non-ribosomal pathway, peptaibols in most cases exist as heterogeneous mixtures of structurally related analogs that have a characteristically high alpha-aminoisobutyric acid content and may contain other non-proteinogenic amino acids or lipoaminoacids (Degenkolb et al. 2007). Mikkola et al. (2012) identified one 11 and eight 20 residue new peptaibols, named trilongins, from a damp building isolate of $T$. longibrachiatum. All tested trilongins formed voltage dependent $\mathrm{Na}^{+} / \mathrm{K}^{+}$permeable channels in boar sperm toxicity assays. They reported $10 \%$ of the dry mycelial mass of the indoor isolate was comprised of these bioactive peptaibols.<smiles>CCCCCc1cccc(=O)o1</smiles>

Simple Trichoderma pyrone<smiles>C/C=C/C=C/C(=O)c1cc(C)c(O)c(C)c1O</smiles>

Sorbicillin

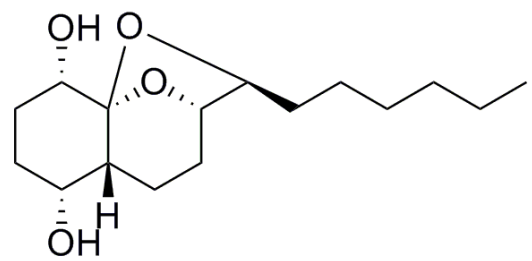

Koninginin A

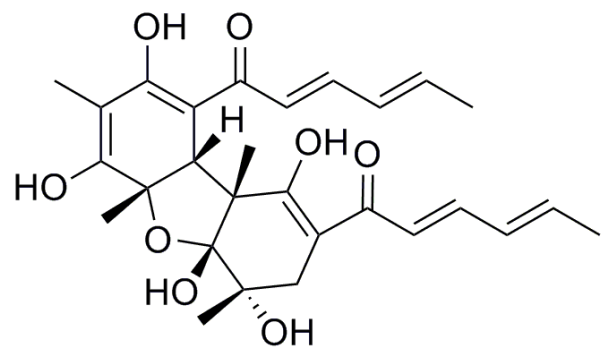

Bisvertinol

Figure 5.4: Structures of the simple pyrone, pyrone 6- pentyl-2H-pyran-2-one, koninginin A, sorbillin and bisvertinol. 


\section{RESULTS AND DISCUSSION}

Nine strains representing three species of Trichoderma were obtained from damp building materials (Table 5.7). DNA was extracted from the mycelia of each indoor isolate, their ITS-1 and 4 sequences were amplified by PCR and each was depositted in the National Mycological

Herbarium culture collection, DAOM. Based on expert analysis of the strains and on a search of the NCBI nucleotide collection database using the BLASTN algorithm with each isolates ITS sequence, five isolates have been identified as T. atroviride, two as T. koningiopsis, one as $T$. citrinoviride and one as T. harzianum (Table 5.7).

All strains were grown on three different liquid media: 2\% malt extract (ME), dextrose casein (DC), and glucose, yeast extract, malt extract, peptone, salts medium (GYMP-salts). Cultures were extracted in a time course manner for each medium to determine the optimal fermentation conditions of each Trichoderma strain. Generally, the GYMP-salts medium after two or four weeks in stationary culture yielded EtOAc culture filtrate extracts with high metabolite yields compared to the other media or fermentation times. The isolation and structural elucidation of the secondary metabolites is discussed below. 


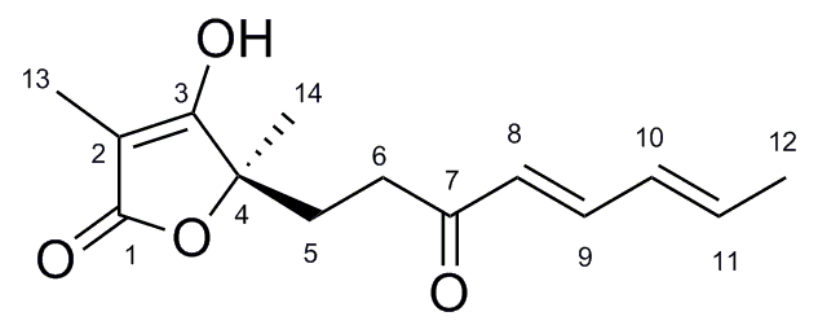

Figure 5.5: Structure of $(R)$ - Vertinolide (5.1).

Table 5.1: ${ }^{1} \mathrm{H}(400 \mathrm{MHz})$ and ${ }^{13} \mathrm{C}$ NMR Data $(100 \mathrm{MHz})$ for $(R)$ - Vertinolide (5.1) in $\mathrm{CD}_{3} \mathrm{OD}$.

\begin{tabular}{ccc}
\hline Position & $\delta_{\mathrm{C}, \text { type }}$ & $\delta_{\mathrm{H}}(J, \mathrm{~Hz})$ \\
\hline $\mathbf{1}$ & $177.5, \mathrm{C}$ & \\
$\mathbf{2}$ & $96.0, \mathrm{C}$ & \\
$\mathbf{3}$ & $180.2, \mathrm{C}$ & \\
$\mathbf{4}$ & $84.2, \mathrm{C}$ & $2.07, \mathrm{~m}$ \\
$\mathbf{5}$ & $32.0, \mathrm{CH}_{2}$ & $2.43, \mathrm{~m}$ \\
$\mathbf{6}$ & $34.9, \mathrm{CH}_{2}$ & $2.52, \mathrm{~m}$ \\
& & \\
$\mathbf{7}$ & $201.7, \mathrm{C}$ & $6.06, \mathrm{~d}(15.6)$ \\
$\mathbf{8}$ & $128.3, \mathrm{CH}$ & $7.17, \mathrm{dd}(9.7,15.6)$ \\
$\mathbf{9}$ & $145.2, \mathrm{CH}$ & $6.27, \mathrm{~m}$ \\
$\mathbf{1 0}$ & $131.5, \mathrm{CH}$ & $6.27, \mathrm{~m}$ \\
$\mathbf{1 1}$ & $142.2, \mathrm{CH}$ & $1.86, \mathrm{~d}(5.2)$ \\
$\mathbf{1 2}$ & $18.9, \mathrm{CH}_{3}$ & $1.64, \mathrm{~s}$ \\
$\mathbf{1 3}$ & $6.0, \mathrm{CH}_{3}$ & $1.42, \mathrm{~s}$ \\
\hline $\mathbf{1 4}$ & $23.6, \mathrm{CH}_{3}$ & \\
\hline
\end{tabular}

$(R)$ - (+)-Vertinolide; (5.1); $6.4 \mathrm{mg}$; yellow amorphous oil; $[\alpha]_{\mathrm{D}} 20(\mathrm{c} 0.3, \mathrm{MeOH}),[\alpha]_{\mathrm{D}} 21(\mathrm{c} 0.3$, $\left.\mathrm{CHCl}_{3}\right) ; \mathrm{UV}(\mathrm{MeOH}) / \mathrm{nm} \lambda_{\max }(\log \varepsilon) 375$ (2.10) 268 (2.67) 230 (2.60); HRESIMS m/z 251.1290 $[\mathrm{M}+\mathrm{H}]^{+}$(calculated for $\mathrm{C}_{14} \mathrm{H}_{19} \mathrm{O}_{4}, 251.1283$ ); ${ }^{1} \mathrm{Hand}{ }^{13} \mathrm{C}$ NMR data were consistent with published data for $(S)$ - vertinolide (Trifonov et al. 1982) and are presented in table 5.1. The structure of $\mathbf{5 . 1}$ can be found in figure 5.5. The optical rotation of compound 5.1 isolated here 
has the same magnitude as (-)- (S)- Vertinolide isolated by Trifonov et al. (1982) however, the sign is opposite; $[\alpha]_{\mathrm{D}}-25\left(\mathrm{c} 0.05, \mathrm{CHCl}_{3}\right)$. As this tetronic acid derivative only has one chiral center, at C-4, the structure of $\mathbf{5 . 1}$ is proposed to be a new fungal metabolite, $(R)$ - vertinolide.

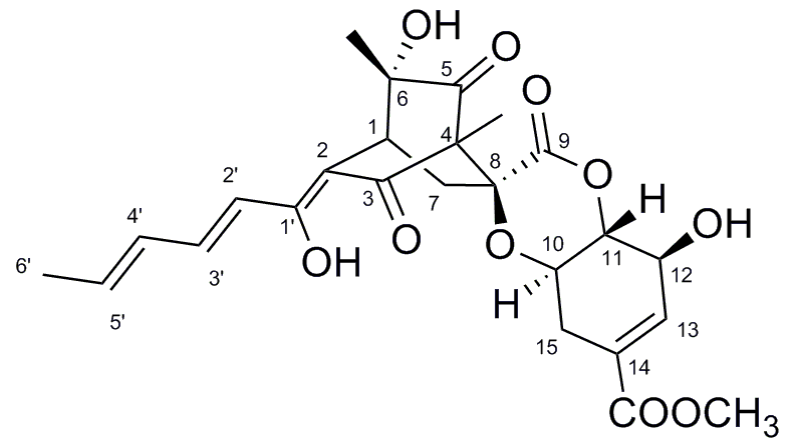

Figure 5.6: Structure of Spirosorbicillinol A (5.2).

Spirosorbicillinol A (5.2); $9.7 \mathrm{mg}$; $[\alpha]_{\mathrm{D}} 125.0$ (c 0.5, MeOH); yellow amorphous oil; UV $(\mathrm{MeOH}) / \mathrm{nm} \lambda_{\max }(\log \varepsilon) 370$ (3.63) 310 (3.73) 255 (3.68) 225 (3.63); HRESIMS m/z 489.1776 $[\mathrm{M}+\mathrm{H}]^{+}$(calculated for $\mathrm{C}_{25} \mathrm{H}_{29} \mathrm{O}_{10}, 489.1760$ ); ${ }^{1}$ Hand ${ }^{13} \mathrm{C}$ NMR data were consistent with published data (Washida et al. 2009) and are presented in table 5.2. The structure of 5.2 can be found in figure 5.6.

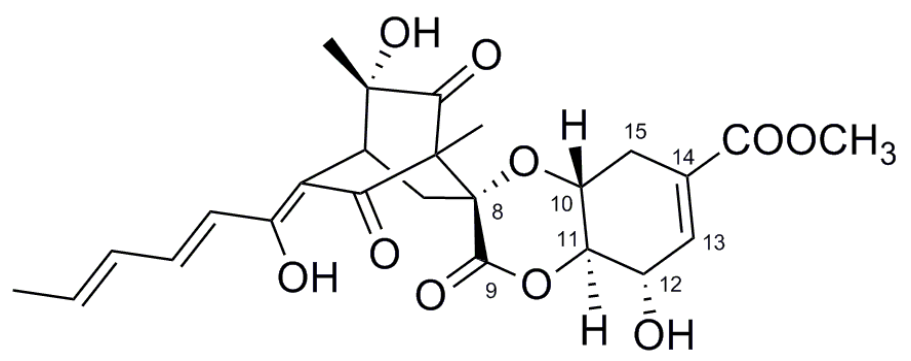

Figure 5.7: Structure of Spirosorbicillinol B (5.3). 
Table 5.2: ${ }^{1} \mathrm{H}(400 \mathrm{MHz})$ and ${ }^{13} \mathrm{C}$ NMR Data $(100 \mathrm{MHz})$ for Spirosorbicillinol A (5.2), B (5.3) and C (5.4) in $\mathrm{CD}_{3} \mathrm{OD}$.

\begin{tabular}{|c|c|c|c|c|c|c|}
\hline \multirow[b]{2}{*}{ Position } & \multicolumn{2}{|c|}{$(5.2)$} & \multicolumn{2}{|c|}{$(5.3)$} & \multicolumn{2}{|c|}{$(5.4)$} \\
\hline & $\delta_{\mathrm{C}, \text { type }}$ & $\delta_{\mathrm{H}}(J, \mathrm{~Hz})$ & $\delta_{\mathrm{C}}$, type & $\delta_{\mathrm{H}}(J, \mathrm{~Hz})$ & $\delta_{\mathrm{C}}$, type & $\delta_{\mathrm{H}}(J, \mathrm{~Hz})$ \\
\hline 1 & $41.4, \mathrm{CH}$ & $3.30, \mathrm{o}$ & $41.2, \mathrm{CH}$ & $3.30, \mathrm{o}$ & $41.3, \mathrm{CH}$ & $3.31, \mathrm{o}$ \\
\hline 2 & $111.2, \mathrm{C}$ & & $111.3, \mathrm{C}$ & & $111.4, \mathrm{C}$ & \\
\hline 3 & $195.6, \mathrm{C}$ & & 196.3, C & & 196.1, C & \\
\hline 4 & $69.3, \mathrm{C}$ & & $71.4, \mathrm{C}$ & & $71.0, \mathrm{C}$ & \\
\hline 5 & $206.8, \mathrm{C}$ & & 207.1, C & & 207.1, C & \\
\hline 6 & $74.6, \mathrm{C}$ & & $75.0, \mathrm{C}$ & & 74.7, C & \\
\hline $7 \mathbf{a}$ & $37.2, \mathrm{CH}_{2}$ & $2.16, \mathrm{dd}(3.0,14.0)$ & $40.3, \mathrm{CH}_{2}$ & $2.32, \mathrm{dd}(2.7,14.1)$ & $40.3, \mathrm{CH}_{2}$ & $2.33, \mathrm{dd}(2.9,14.0)$ \\
\hline & & $3.04, \mathrm{dd}(3.0,14.0)$ & & $2.98, \mathrm{dd}(2.7,14.1)$ & & 2.97, dd $(2.9,14.0)$ \\
\hline 8 & $84.3, \mathrm{C}$ & & $82.6, \mathrm{C}$ & & $83.8, \mathrm{C}$ & \\
\hline 9 & $172.0, \mathrm{C}$ & & $171.0, \mathrm{C}$ & & $171.3, \mathrm{C}$ & \\
\hline 10 & $67.1, \mathrm{CH}$ & $6.06, \mathrm{~m}$ & $71.4, \mathrm{CH}$ & $4.01, \mathrm{~m}$ & $67.2, \mathrm{CH}$ & $4.02, \mathrm{~m}$ \\
\hline 11 & $85.9, \mathrm{CH}$ & $4.25, \mathrm{dd}(8.1,10.0)$ & $84.3, \mathrm{CH}$ & $4.36, \mathrm{dd}(7.9,10.1)$ & $82.9, \mathrm{CH}$ & 4.30, dd $(8.6,10.1)$ \\
\hline 12 & $70.6, \mathrm{CH}$ & $4.44, \mathrm{~m}$ & $70.7, \mathrm{CH}$ & $4.44, \mathrm{~m}$ & $74.8, \mathrm{CH}$ & $4.47, \mathrm{~m}$ \\
\hline 13 & $139.6, \mathrm{CH}$ & $6.64, \mathrm{t}(2.5)$ & $139.3, \mathrm{CH}$ & $6.63, \mathrm{t}(2.1)$ & $134.2, \mathrm{CH}$ & $6.62, \mathrm{bs}$ \\
\hline 14 & $129.0, \mathrm{C}$ & & $128.8, \mathrm{C}$ & & $130.8, \mathrm{C}$ & \\
\hline 15 & $30.6, \mathrm{CH}_{2}$ & $2.24, \mathrm{~m}$ & $31.5, \mathrm{CH}_{2}$ & $2.27, \mathrm{~m}$ & $34.1, \mathrm{CH}_{2}$ & $2.25, \mathrm{~m}$ \\
\hline & & $2.79, \mathrm{dd}(6.6,17.4)$ & & $2.89, \mathrm{dd}(6.5,17.8)$ & & $2.89, \mathrm{dd}(6.7,18.1)$ \\
\hline 1 ' & $168.3, \mathrm{C}$ & & $167.9, \mathrm{C}$ & & 167.3, C & \\
\hline 2' & $119.3, \mathrm{CH}$ & $6.41, \mathrm{~d}(15.0)$ & $119.5, \mathrm{CH}$ & $6.36, \mathrm{~d}(14.9)$ & $119.5, \mathrm{CH}$ & $6.38, \mathrm{~d}(15.2)$ \\
\hline 3 ' & 143.7, $\mathrm{CH}$ & 7.32, dd $(10.9,14.9)$ & $143.2, \mathrm{CH}$ & 7.28, dd $(10.9,15.0)$ & $143.3, \mathrm{CH}$ & 7.29, dd $(10.9,14.9)$ \\
\hline 4 ' & 132.3, $\mathrm{CH}$ & $6.38, \mathrm{~m}$ & $132.3, \mathrm{CH}$ & $6.38, \mathrm{~m}$ & 132.3, $\mathrm{CH}$ & $6.38, \mathrm{~m}$ \\
\hline 5 & $140.6, \mathrm{CH}$ & $6.22, \mathrm{~m}$ & $140.1, \mathrm{CH}$ & $6.20, \mathrm{~m}$ & $140.1, \mathrm{CH}$ & $6.21, \mathrm{~m}$ \\
\hline $6^{\prime}$ & $18.9, \mathrm{CH}_{3}$ & $1.87, \mathrm{~d}(6.7)$ & $18.8, \mathrm{CH}_{3}$ & $1.87, \mathrm{~d}(6.7)$ & $18.9, \mathrm{CH}_{3}$ & $1.87, \mathrm{~d}(6.7)$ \\
\hline $4-\mathrm{CH}_{3}$ & $8.1, \mathrm{CH}_{3}$ & $1.22, \mathrm{~s}$ & $8.8, \mathrm{CH}_{3}$ & $1.24, \mathrm{~s}$ & $8.6, \mathrm{CH}_{3}$ & $1.24, \mathrm{~s}$ \\
\hline $6-\mathrm{CH}_{3}$ & $24.8, \mathrm{CH}_{3}$ & $1.19, \mathrm{~s}$ & $24.8, \mathrm{CH}_{3}$ & $1.18, \mathrm{~s}$ & $24.8, \mathrm{CH}_{3}$ & $1.19, \mathrm{~s}$ \\
\hline 14- $\underline{\mathrm{COOCH}} 3$ & $167.4, \mathrm{C}$ & & $167.4, \mathrm{C}$ & & $167.3, \mathrm{C}$ & \\
\hline 14- $\mathrm{COOCH}_{3}$ & $52.7, \mathrm{CH}_{3}$ & $3.74, \mathrm{~s}$ & $52.6, \mathrm{CH}_{3}$ & $3.74, \mathrm{~s}$ & $52.7, \mathrm{CH}_{3}$ & $3.73, \mathrm{~s}$ \\
\hline
\end{tabular}

- Overlapped with signal from $\mathrm{CD}_{3} \mathrm{OD}$. 
Spirosorbicillinol B (5.3); 4.7 mg; yellow amorphous oil; [ $\alpha]_{\mathrm{D}} 265.4$ (c 0.25, MeOH); UV $(\mathrm{MeOH}) / \mathrm{nm} \lambda_{\max }(\log \varepsilon) 370$ (4.05) 310 (4.14) 255 (4.10) 225 (4.08); HRESIMS m/z 489.1775 $[\mathrm{M}+\mathrm{H}]^{+}$(calculated for $\mathrm{C}_{25} \mathrm{H}_{29} \mathrm{O}_{10}, 489.1760$ ); ${ }^{1} \mathrm{Hand}{ }^{13} \mathrm{C}$ NMR data were consistent with published data (Washida et al. 2009) and are presented in table 5.2. The structure of $\mathbf{5 . 3}$ can be found in figure 5.7 .

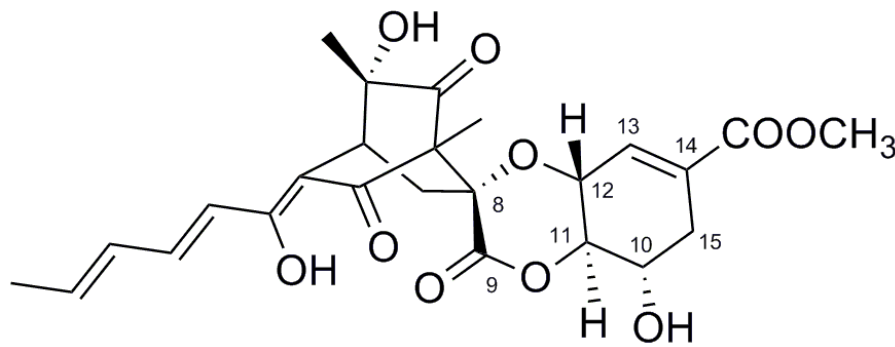

Figure 5.8: Structure of Spirosorbicillinol C (5.4).

Spirosorbicillinol C (5.4); 5.0 mg; yellow amorphous oil; [ $\alpha]_{\mathrm{D}} 320.5$ (c 0.25, MeOH); UV $(\mathrm{MeOH}) / \mathrm{nm} \lambda_{\max }(\log \varepsilon) 370$ (3.89) 310 (3.93) 255 (3.78) 225 (3.76); HRESIMS m/z 489.1773 $[\mathrm{M}+\mathrm{H}]^{+}$(calculated for $\mathrm{C}_{25} \mathrm{H}_{29} \mathrm{O}_{10}, 489.1760$ ); ${ }^{1}$ Hand ${ }^{13} \mathrm{C}$ NMR data were consistent with published data (Washida et al. 2009) and are presented in table 5.2. The structure of 5.4 can be found in figure 5.8.

Trichotetronine (5.5), $11.1 \mathrm{mg}$; yellow amorphous oil; $[\alpha]_{\mathrm{D}} 80$ (c 0.55, MeOH; UV (MeOH)/nm $\lambda_{\max }(\log \varepsilon) 370$ (4.45) 293 (4.60) 260 (4.52); HRESIMS $m / z 497.2191[\mathrm{M}+\mathrm{H}]^{+}$(calculated for $\mathrm{C}_{28} \mathrm{H}_{33} \mathrm{O}_{8}, 497.2175$ ); ${ }^{1}$ Hand ${ }^{13} \mathrm{C}$ NMR data were consistent with published data (Maskey et al. 2005) and are presented in table 5.3. The structure of $\mathbf{5 . 3}$ can be found in figure 5.9. 


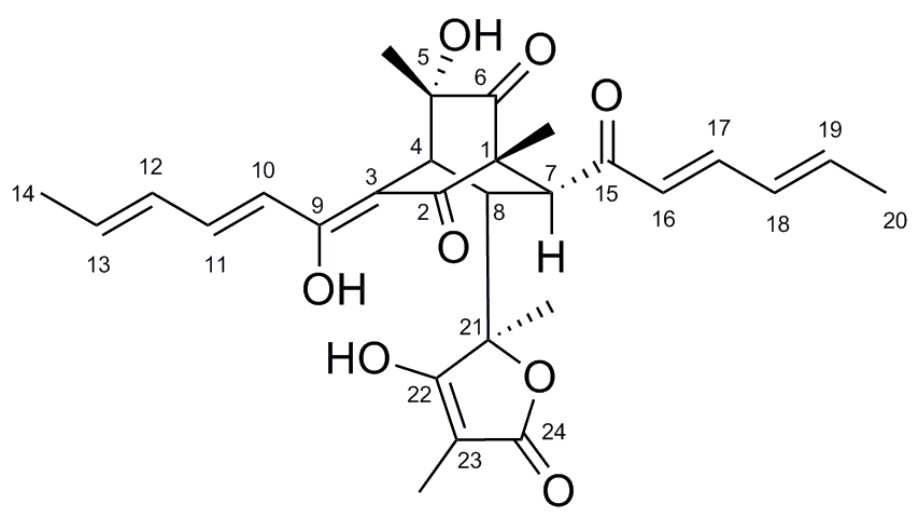

Figure 5.9: Structure of Trichotetronine (5.5).

Table 5.3: ${ }^{1} \mathrm{H}(400 \mathrm{MHz})$ and ${ }^{13} \mathrm{C}$ NMR Data $(100 \mathrm{MHz})$ for Trichotetronine (5.5) in $\mathrm{CD}_{3} \mathrm{OD}$.

\begin{tabular}{|c|c|c|}
\hline Position & $\delta_{\mathrm{C}}$, type & $\delta_{\mathrm{H}}(J, \mathrm{~Hz})$ \\
\hline 1 & $63.6, \mathrm{C}$ & \\
\hline 2 & 197.5, C & \\
\hline 3 & 110.1, C & \\
\hline 4 & $43.5, \mathrm{CH}$ & $3.30, \mathrm{o}$ \\
\hline 5 & 75.8, C & \\
\hline 6 & 210.1, C & \\
\hline 7 & $52.8, \mathrm{CH}$ & $3.28, \mathrm{o}$ \\
\hline 8 & $43.9, \mathrm{CH}$ & $3.29, \mathrm{o}$ \\
\hline 9 & $169.8, \mathrm{C}$ & \\
\hline 10 & 119.6, CH & $6.28, \mathrm{~m}$ \\
\hline 11 & $143.8, \mathrm{CH}$ & $7.30, \mathrm{dd}(11,14.5)$ \\
\hline 12 & $132.4, \mathrm{CH}$ & $6.36, \mathrm{~m}$ \\
\hline 13 & $140.7, \mathrm{CH}$ & $6.22, \mathrm{~m}$ \\
\hline 14 & $18.9, \mathrm{CH}_{3}$ & $1.90, \mathrm{~d}(6.8)$ \\
\hline 15 & $202.5, \mathrm{C}$ & \\
\hline 16 & $128.5, \mathrm{CH}$ & $6.25, \mathrm{~m}$ \\
\hline 17 & $147.8, \mathrm{CH}$ & $7.19, \mathrm{dd}(10.4,15.2)$ \\
\hline 18 & 131.6, CH & $6.36, \mathrm{~m}$ \\
\hline 19 & $145.0, \mathrm{CH}$ & $6.38, \mathrm{~m}$ \\
\hline 20 & $19.1, \mathrm{CH}_{3}$ & $1.90, \mathrm{~d}(6.8)$ \\
\hline 21 & $84.5, \mathrm{C}$ & \\
\hline 22 & 177.1, C & \\
\hline 23 & $97.1, \mathrm{C}$ & \\
\hline 24 & $180.1, \mathrm{C}$ & \\
\hline $1-\mathrm{CH}_{3}$ & $11.3, \mathrm{CH}_{3}$ & $0.98, \mathrm{~s}$ \\
\hline $5-\mathrm{CH}_{3}$ & $24.2, \mathrm{CH}_{3}$ & $1.17, \mathrm{~s}$ \\
\hline $21-\mathrm{CH}_{3}$ & $23.5, \mathrm{CH}_{3}$ & $1.40, \mathrm{~s}$ \\
\hline $23-\mathrm{CH}_{3}$ & $6.6, \mathrm{CH}_{3}$ & $1.48, \mathrm{~s}$ \\
\hline
\end{tabular}

o- Overlapped with signal from $\mathrm{CD}_{3} \mathrm{OD}$. 
Bisvertinol (5.6); $7.0 \mathrm{mg}$; dark yellow amorphous oil; $[\alpha]_{\mathrm{D}}-1220$ (c 0.25, MeOH); UV $(\mathrm{MeOH}) / \mathrm{nm} \lambda_{\max }(\log \varepsilon) 410$ (4.06) 300 (4.20) 278 (4.20) 230 (4.01); HRESIMS m/z 499.2345 $[\mathrm{M}+\mathrm{H}]^{+}$(calculated for $\mathrm{C}_{28} \mathrm{H}_{35} \mathrm{O}_{8}, 499.2332$ ); ${ }^{1}$ Hand ${ }^{13} \mathrm{C}$ NMR data were consistent with published data (Trifinov et al. 1986; Koyama et al. 2007) and are presented in table 5.4. The structure of $\mathbf{5 . 3}$ can be found in figure 5.10.

Table 5.4: ${ }^{1} \mathrm{H}(400 \mathrm{MHz})$ and ${ }^{13} \mathrm{C}$ NMR Data $(100 \mathrm{MHz})$ for Bisvertinol (5.6) in $\mathrm{CD}_{3} \mathrm{CN}$.

\begin{tabular}{|c|c|c|}
\hline Position & $\delta_{\mathrm{C}}$, type & $\delta_{\mathrm{H}}(J, \mathrm{~Hz})$ \\
\hline 1 & $167.5, \mathrm{C}$ & \\
\hline 2 & $105.8, \mathrm{C}$ & \\
\hline \multirow[t]{2}{*}{3} & $36.2, \mathrm{CH}_{2}$ & $2.49, \mathrm{~d}(14.4)$ \\
\hline & & $2.62, \mathrm{~d}(14.4)$ \\
\hline 4 & 73.7, C & \\
\hline $4 a$ & 102.1, C & \\
\hline $5 \mathbf{a}$ & $80.0, \mathrm{C}$ & \\
\hline 6 & $180.2, \mathrm{C}$ & \\
\hline 7 & 109.7, C & \\
\hline 8 & $168.9, \mathrm{C}$ & \\
\hline 9 & $106.9, \mathrm{C}$ & \\
\hline $9 a$ & $54.6, \mathrm{CH}$ & $3.62, \mathrm{~s}$ \\
\hline $9 b$ & $59.5, \mathrm{C}$ & \\
\hline 10 & $193.4, \mathrm{C}$ & \\
\hline 11 & $121.8, \mathrm{CH}$ & $6.51, \mathrm{~d}(14.6)$ \\
\hline 12 & $142.8, \mathrm{CH}$ & $7.22, \mathrm{~m}$ \\
\hline 13 & 131.6, CH & $6.36, \mathrm{~m}$ \\
\hline 14 & $138.8, \mathrm{CH}$ & $7.17, \mathrm{~m}$ \\
\hline 15 & $18.9, \mathrm{CH}_{3}$ & $1.85, \mathrm{~d}(6.4)$ \\
\hline 16 & $22.3, \mathrm{CH}_{3}$ & $1.13, \mathrm{~s}$ \\
\hline 17 & $25.9, \mathrm{CH}_{3}$ & $1.40, \mathrm{~s}$ \\
\hline 18 & $7.2, \mathrm{CH}_{3}$ & $1.38, \mathrm{~s}$ \\
\hline 19 & $192.6, \mathrm{C}$ & \\
\hline 20 & 121.6, CH & $6.35, \mathrm{~m}$ \\
\hline 21 & $140.8, \mathrm{CH}$ & $6.24, \mathrm{~m}$ \\
\hline 22 & $132.0, \mathrm{CH}$ & $6.36, \mathrm{~m}$ \\
\hline 23 & $137.4, \mathrm{CH}$ & $6.13, \mathrm{~m}$ \\
\hline 24 & $18.7, \mathrm{CH}_{3}$ & $1.85, \mathrm{~d}(6.4)$ \\
\hline 25 & $19.7, \mathrm{CH}_{3}$ & $1.25, \mathrm{~s}$ \\
\hline
\end{tabular}




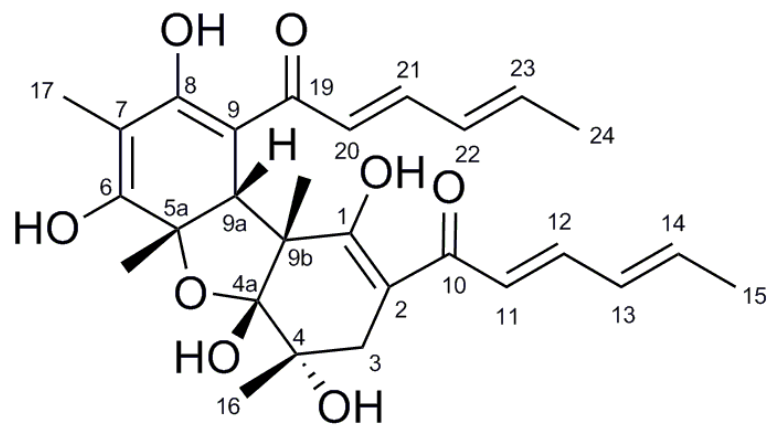

Figure 5.10: Structure of Bisvertinol (5.6).

All of these metabolites (5.1-5.6) were isolated from the EtOAc culture filtrate extract of $T$. citrinoviride (Paracel Number- 1237071-1) obtained from an indoor air sample in Montreal, QC. Large scale fermentations, $10 \mathrm{~L}$, were done in Glaxo bottles containing 1L of GYMP-salts media (refer to materials and methods) and were extracted after 2 weeks.

Vertinolide (5.1) was originally isolated as the major constituent of the chloroform extract of Verticillium intertextum culture filtrate by Trifonov et al. (1982). This is a synonym of Clonostachys rosea formerly called Gliocladium roseum (http://www.mycobank.org/). They determined the structure by X-ray crystallography and chemical derivatization however the stereochemistry at the C-4 position remained obscure. The absolute configuration was determined to be $(S)$ by the synthesis of $(+)$ tetrahydrovertinolide from (-) valerolactonecarboxylic acid by Takaiwa and Yamashita (1983). Vertinolide is a tetronic acid metabolite that is a subclass of beta-hydroxybutenolides. These natural products are ecologically important as they are flavoring components of fruits, mycotoxins or possess desirable bioactivities including antioxidant, antibiotic, herbicidal, or insecticidal (Wrobel and Ganem 1983). Abdel-Lafeff et al. 
(2009) reported the isolation of vertinolide with bisvertinol, trichodimerol, trichotetronine, trichodermanones A-D and a new pyranone derivative, trichopyrone, from T. viride isolated from the marine sponge Agelas dispar.

The metabolite profile described by Abdel-Lafeff et al. (2009) is more consistent with that of $T$. citrinoviride or possibly the related T. longibrachiatum as opposed to $T$. viride. Many of the metabolites they isolated were also found in this T. citrinoviride work, previous investigations of the metabolites of T. citrinoviride (Evidente et al. 2009) or T. longibrachiatum (Andrade et al. 1992; 1996). Compounds isolated by Abdel-Lafeff et al. (2009) were investigated for radical scavenging and inhibition of HIV-1 reverse transcriptase and were found to be relatively inactive in all bioassays. 5-hydroxyvertinolide was isolated from the related species T. longibrachiatum by Andrade et al. (1992).

Compound $\mathbf{5 . 5}$ was structurally characterized by three groups at roughly the same time and received three different names; bisorbutenolide (Abe et al. 1998), bislongiquinolide (Andrade et al. 1997) and trichotetronine (Shirota et al. 1997). For simplicity's sake, it will be referred to as trichotetronine here. This metabolite bears a tetronic acid moiety as it is derived from vertinolide and sorbicillin. Abe et al. (1998) isolated trichotetronine, called bisorbutenolide, from Trichoderma USF-2690, a strain obtained from a soil sample. They also isolated trichodimerol and bisorbicillinolide. These latter two metabolites were not isolated here. More recently, Maskey et al. (2005) demonstrated by NMR that there is a stereochemical difference between what has been referred to as trichotetronine, $21 R,(\mathbf{5 . 5})$ and bisorbutenolide, $21 S$, isolated by Abe et al. (1998). The NRM data of compound 5.5 isolated here is consistent with that of the $21 R$ 
isomer, trichotetronine (Maskey et al. 2005). This chiral center, C-21, is the sole stereogenic carbon within the vertinolide moiety of $\mathbf{5 . 5}$ that was determined to be of the $R$ configuration based on the optical rotation of 5.1. This further suggests that 5.1, $R$-vertinolide, is a new metabolite and is incorporated during the biosynthesis of 5.5. All of these sorbicillin derived metabolites were assessed for antioxidant activity by Abe et al. (1998). Trichotetronine (5.5) displayed DPPH-radical scavenging activity with an $\mathrm{ED}_{50}$ of $80.8 \mu \mathrm{M}$.

Andrade et al. (1997) isolated bislongiquinolide from Trichoderma longibrachiatum, which is antagonistic to the causative agent of the American leaf spot disease of coffee. Shirota et al. (1997) reported trichotetronine with its dihydrocongener from a Trichoderma species isolated from rice plant straw. Maskey et al. (2005) identified trichotetronine with various other vertinolide derived metabolites from Penicillium notatum (= P. chrysogenum) obtained from a bench top contamination. Isolated metabolites were semi-quantitatively tested using a agar diffusion test against a variety of bacteria. Most metabolites tested exhibited weak antibacterial activity where compound $\mathbf{5 . 5}$ had no activity; fungi and algae were not inhibited by any of these compounds.

Spirosorbicillinols A-C (5.2-5.4) were characterized for the first time from the fermentation broth of an unidentified Trichoderma strain isolated from a soil sample collected in Japan. Other sorbicillin derived metabolites were also isolated. The antioxidant activity of 5.2-5.4 were assessed by DPPH-radical scavenging and displayed weak activity, the $\mathrm{ED}_{50}$ values for spirosorbicillinol A-C were 190, 236 and $321 \mu \mathrm{M}$ respectively (Washida et al. 2009). Each of the spirosorbicillinols characterized here were isolated in similar yields. 
Bisvertinol (5.6) was isolated for the first time from the culture of Verticillium intertextum by Trifonov et al. (1986). Bisvertinol was also isolated from T. longibrachiatum with its oxidized form, bisvertinolone. This metabolite elicits anti-fungal activity by inhibiting $\beta$-1, 6-glucan biosynthesis (Andrade et al. 1992; Kontani et al. 1994). Bisvetinol, isobisvertinol, and dihydrobisvertinol have been characterized from an Aspergillus strain collected from a mangrove soil in Japan. These metabolites were assayed for cholesteryl ester and triacylglycerol synthase inhibition in mice macrophages. Isobisvertinol had $\mathrm{IC}_{50}$ values of 2.5 and $4.0 \mu \mathrm{M}$ for for cholesteryl ester and triacylglycerol synthase respectively. Bisvertinol and dihydrobisvertinol showed almost no effect. This suggested that the stereochemical difference at C-9a is important for binding to a target molecule and eliciting this activity (Koyama et al. 2007).

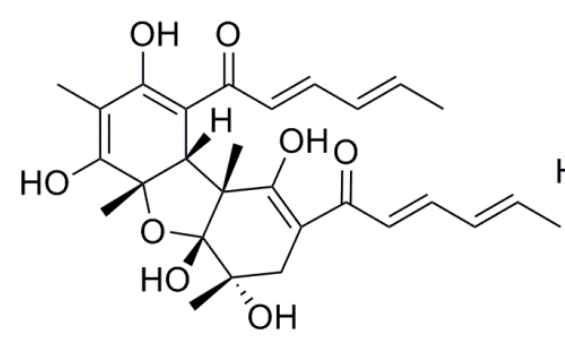

Bisvertinol

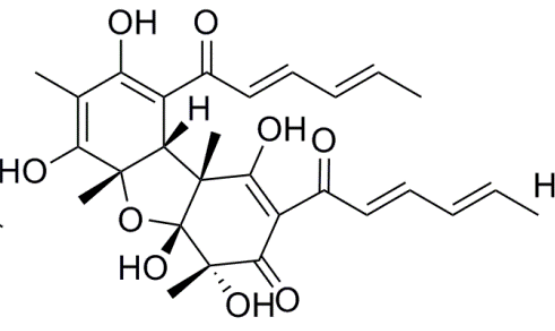

Bisvertinolone

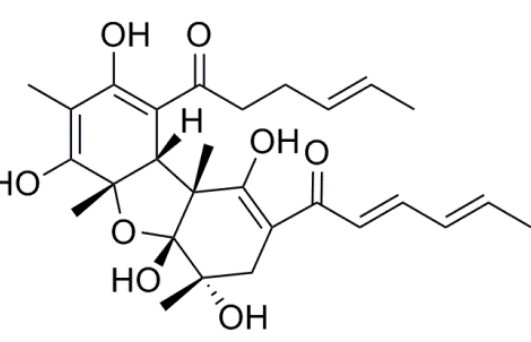

Dihydrobisvertinol

Figure 5.11: Structure of bisvertinol, bisvertinolone and dihydrobisvertinol.

Bisorbicillinoids are an expanding group of natural products that have interesting biological properties due to their complex structural framework. They have been reliably isolated from Vericillium intertextum, Penicillium species and Trichoderma species. All vertinolides exhibit radical scavenging activity, some dimers inhibit the $\beta-1,6$-glucan biosynthesis or have weak 
cytotoxicity against human tumor cells (Maskey et al. 2005). This structural complexity and biological activity has prompted the exploration of their biosynthesis by various labeled precursor and synthetic studies.

These natural products belong to the sorbicillin class that is derived from a hexaketide moiety where a cyclization occurs at the carboxylate terminus. Sorbicillin initially undergoes an oxidative dearomatization that generates the key intermediate sorbicillinol which is composed of a conjugated six membered ring bearing a sorbyl functionality. It has been postulated that sorbicillionol is the common precursor for this class of secondary metabolites. It can undergo various tautomerization generating various reactive sites making sorbicillin-derived metabolites difficult to isolate in high purity from fermentation broth. The cyclohexanone ring may also be further methylated or hydroxylated. To increase structural diversity, sorbicillinol may undergo various transformations to produce monomeric, homo and hetero (vertinolides) dimeric or trimeric compounds.
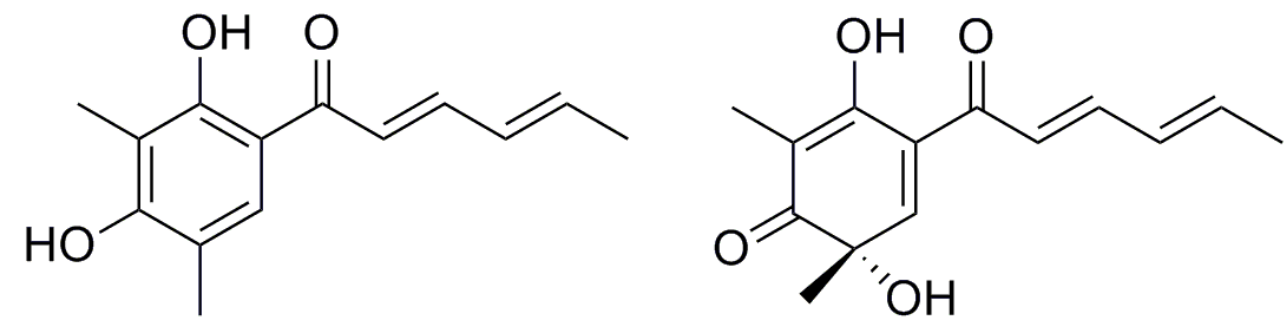

Figure 5.12: Structure of sorbicillin and sorbicillinol.

The ortho-quinol system present in sorbicillinol acts as a pair of diene-dienophile in a Diels Alder reaction in a regio- and stereo- specific manner. Intramolecular rearrangement of the 
cycloaddition adduct generates various secondary metabolites including bisorbibutenolide and bisorbicillinolide. Bridged bicyclic bisorbillinoids are produced through a [4+2] cycloaddition providing the bicyclo[2.2.2] octanedione ring system. When the ortho-quinol system acts as a Michael donor-Michael acceptor pair, after rearrangement it may produce a fused [6.5.6] or cage like cores observed in trichodimerol and bisvertinol respectively. The monomeric vertinolides series of compounds can be derived by a series of transformation such as lactonization, ring cleavage reduction and oxidation. These postulated biosynthetic pathways have been confirmed from feeding experiments (Sugaya et al. 2008; Abe et al. 2001; 2002) and biomimetic synthetic studies (Nicolaou et al. 1999).

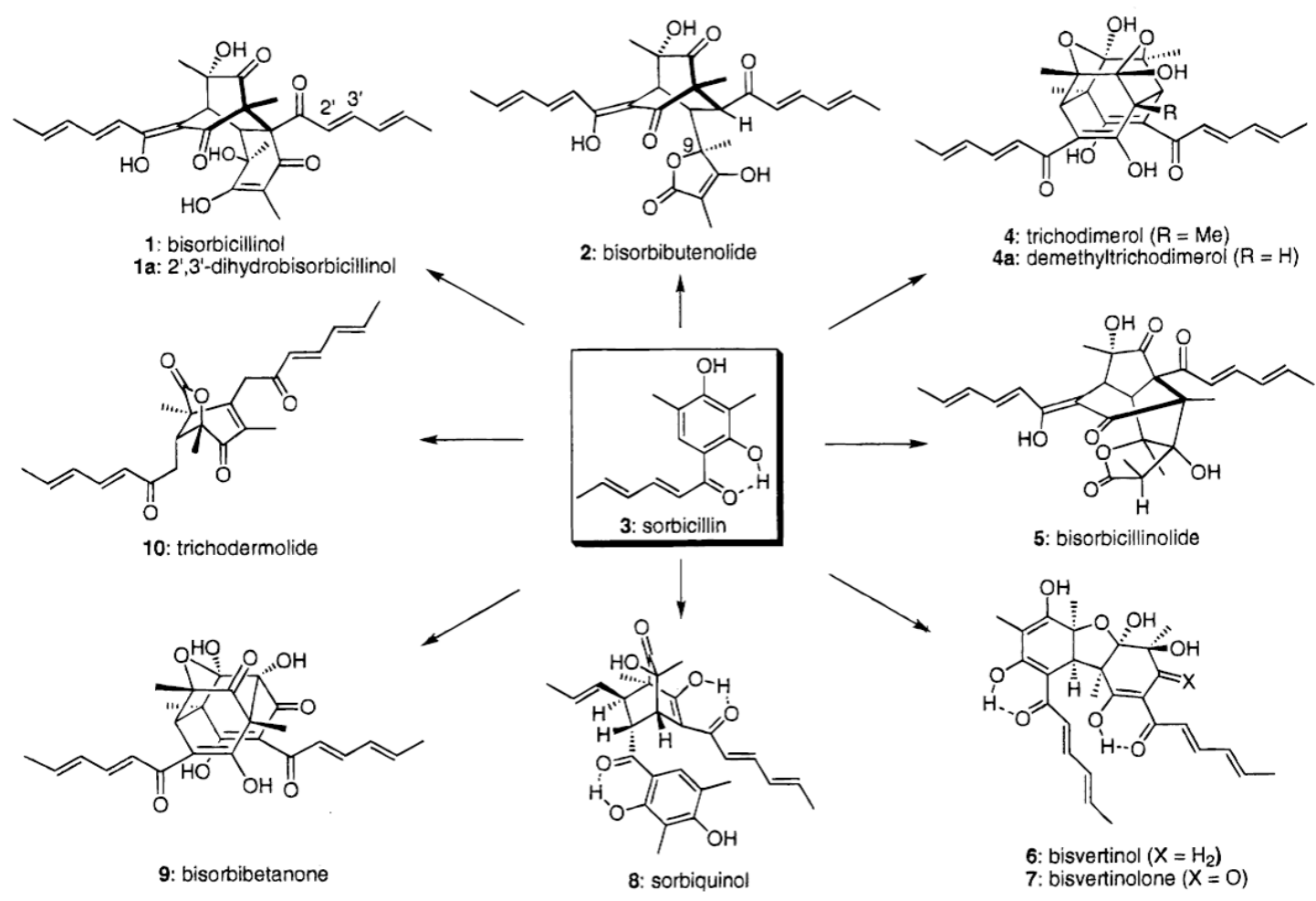


Figure 5.13: Structure of various bisorbicillinoids derived from their postulated precursor sorbicillin illustrating their structural diversity (adapted from Nicolaou et al. 1999).

Other metabolites from $T$. citrinoviride include antifungal polypeptides from a strain endophytic in Mediterranean Cork (Maddau et al. 2009) as well as the insect anti-feedant compounds citrantifidiene and citrantifidiol (Evidente et al. 2008). Maddau et al. (2009) identified twenty eight 20 residue peptides, peptaibols, with structural variation at positions $6,9,12$ and 17 . The peptide mixture displayed strong antifungal activity toward seven important forest tree pathogens with up to $70 \%$ inhibition at $50 \mathrm{ug} \mathrm{mL}^{-1}$ and it was highly toxic in a brine shrimp bioassay, $\mathrm{LC}_{50}$ $=1.24 \mathrm{ug} \mathrm{mL} \mathrm{m}^{-1}$. Citrantifidiene and citrantifidiol were isolated from a T. citrinoviride isolate obtained from an Austrian soil sample. These two endophytic compounds were reported to have antifeedant activity against the aphid pest Schizaphis graminum when dipping leaves in a 5\% aqueous methanol solution containing $0.57 \mathrm{mg} \mathrm{mL}^{-1}$ of citrantifidiene and $0.91 \mathrm{mg} \mathrm{mL}^{-1}$ citrantifidiol (Evidente et al. 2008). In a separate study, the same authors reported the isolation of the four bisorbicillinoids: trichodimerol, dihydrotrichodimerol, bislongiquinolide and dihydrobislongiquinolide. Dihydrotrichodimerol and bislongiquinolide additionally demonstrated antifeedant activity towards $S$. graminum, restraining the aphids from settling on leaves treated with these two metabolites (Evidente et al. 2009).
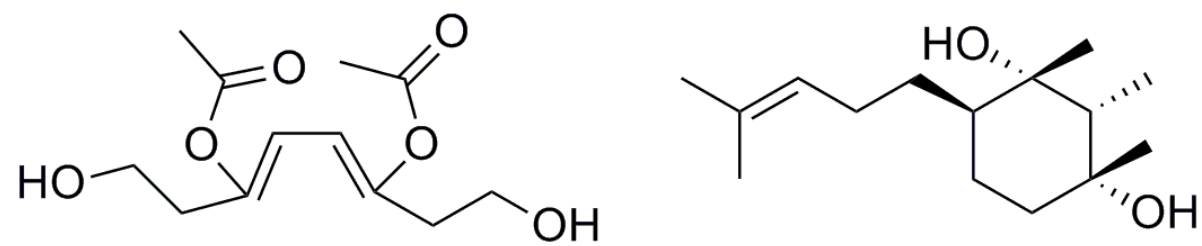

Figure 5.14: Structure of citrantifidiene and citrantifidiol isolated from T. citrinoviride. 


\section{Secondary Metabolites from Trichoderma koningiopsis}

Koninginin A (5.7); $17.9 \mathrm{mg}$; light yellow solid; $[\alpha]_{\mathrm{D}}-27.3$ (c 0.80, MeOH); UV (MeOH)/nm $\lambda_{\max }(\log \varepsilon) 210$ (1.75), 262 (2.24); HRESIMS $m / z$ 285. $2072[\mathrm{M}+\mathrm{H}]^{+}$(calculated for $\mathrm{C}_{16} \mathrm{H}_{29} \mathrm{O}_{4}$, 285.2066). ${ }^{1} \mathrm{H}$ and ${ }^{13} \mathrm{C}$ NMR data for 5.7 isolated from T. koningiopsis were consistent with published data (Cutler et al. 1989) and are also reported in table 5.5. The structure of koninginin A (5.7 or 3.12) can be found in figure 3.21; this metabolite was previously isolated from $P$. corylophilum during this work.

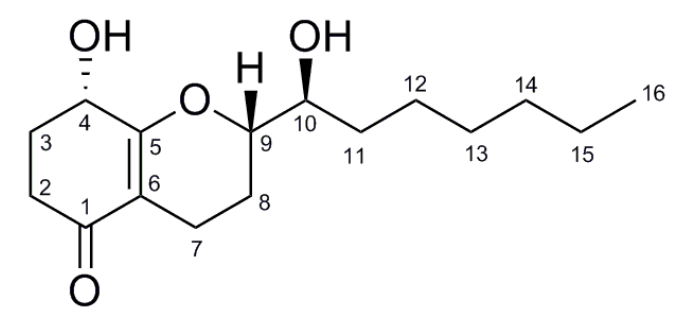

Figure 5.15: Structure of Koninginin B (5.8).

Koninginin B (5.8); 21.9 mg; light yellow solid; $[\alpha]_{\mathrm{D}} 150.0$ (c 1.0, MeOH); UV (MeOH)/nm $\lambda_{\max }$ (log $\varepsilon) 210$ (2.71), 260 (2.88); HRESIMS $m / z$ 283. $1915[\mathrm{M}+\mathrm{H}]^{+}$(calculated for $\mathrm{C}_{16} \mathrm{H}_{27} \mathrm{O}_{4}$, 283.1909). ${ }^{1} \mathrm{H}$ and ${ }^{13} \mathrm{C}$ NMR data were consistent with published data (Cutler et al. 1991) and are reported in table 5.5. The structure of koninginin B (5.8) can be found in figure 5.15. Koninginin B was differentiated from its C-4 epimer koninginin $\mathrm{E},[\alpha]_{\mathrm{D}}+8.3(\mathrm{c} 0.30, \mathrm{MeOH})$, by optical rotation. The reported optical rotations for koninginin E and B were 0.6 and 162.9, respectively (Cutler et al. 1991; Parker et al. 1995). 
Table 5.5: ${ }^{1} \mathrm{H}(400 \mathrm{MHz})$ and ${ }^{13} \mathrm{C}$ NMR Data $(100 \mathrm{MHz})$ for koninginins $\mathrm{A}(\mathbf{5 . 7}), \mathrm{B}(\mathbf{5 . 8}), \mathrm{D}(\mathbf{5 . 9})$ and $\mathrm{F}(\mathbf{5 . 1 0})$ in $\mathrm{CD}$ OD.

\begin{tabular}{|c|c|c|c|c|c|c|c|c|}
\hline \multirow[b]{2}{*}{ Position } & \multicolumn{2}{|r|}{$(5.7)$} & \multicolumn{2}{|r|}{$(5.8)$} & \multicolumn{2}{|r|}{ (5.9) } & \multicolumn{2}{|c|}{$(5.10)$} \\
\hline & $\delta_{\mathrm{C}}$ type & $\delta_{\mathrm{H}}(J, \mathrm{~Hz})$ & $\delta_{\mathrm{C}}$ type & $\delta_{\mathrm{H}}(J, \mathrm{~Hz})$ & $\delta_{\mathrm{C}}$, type & $\delta_{\mathrm{H}}(J, \mathrm{~Hz})$ & $\delta_{\mathrm{C}}$, type & $\delta_{\mathrm{H}}(J, \mathrm{~Hz})$ \\
\hline 1 & $80.2, \mathrm{CH}$ & $4.26, \mathrm{~s}$ & 200.4 & & $199.5, \mathrm{C}$ & & $199.1, \mathrm{C}$ & \\
\hline 2 & $28.1, \mathrm{CH}_{2}$ & $\begin{array}{l}1.49, \mathrm{~m} \\
2.17, \mathrm{~m}\end{array}$ & $33.5, \mathrm{CH}_{2}$ & $\begin{array}{c}2.21, \mathrm{~m} \\
2.27, \operatorname{ddd}(4.8,6.6,16.9)\end{array}$ & 34.1, $\mathrm{CH}_{2}$ & $2.30, \mathrm{~m}$ & $72.4, \mathrm{CH}$ & $4.08, \mathrm{~m}$ \\
\hline 3 & 21.7, $\mathrm{CH}_{2}$ & $\begin{array}{l}1.63, \mathrm{~m} \\
2.13, \mathrm{~m}\end{array}$ & $30.3, \mathrm{CH}_{2}$ & $1.97, \mathrm{~m}$ & $30.4, \mathrm{CH}_{2}$ & $\begin{array}{l}1.98, \mathrm{~m} \\
2.17, \mathrm{~m}\end{array}$ & $33.0, \mathrm{CH}_{2}$ & $\begin{array}{l}2.16, \mathrm{~m} \\
2.24, \mathrm{~m}\end{array}$ \\
\hline 4 & $80.5, \mathrm{CH}$ & $4.04, \mathrm{t}(6.5)$ & $66.6, \mathrm{CH}$ & $4.35, \mathrm{t}(4.9)$ & $66.8, \mathrm{CH}$ & $4.39, \mathrm{~m}$ & $32.8, \mathrm{CH}_{2}$ & $\begin{array}{l}2.54, \mathrm{~m} \\
2.67, \mathrm{~m}\end{array}$ \\
\hline 5 & $110.1, \mathrm{C}$ & & $172.7, \mathrm{C}$ & & $174.2, \mathrm{C}$ & & $174.7, \mathrm{C}$ & \\
\hline 6 & $43.1, \mathrm{CH}$ & $1.55, \mathrm{~m}$ & $112.3, \mathrm{C}$ & & $114.4, \mathrm{C}$ & & $113.1, \mathrm{C}$ & \\
\hline 7 & $36.1, \mathrm{CH}$ & $\begin{array}{l}1.45, \mathrm{~m} \\
1.60, \mathrm{~m}\end{array}$ & $18.7, \mathrm{CH}_{2}$ & $2.09, \mathrm{~m}$ & $57.6, \mathrm{CH}$ & $4.64, \mathrm{~m}$ & $57.7, \mathrm{CH}$ & 4.73, m \\
\hline 8 & $27.4, \mathrm{CH}_{2}$ & $1.83, \mathrm{~m}$ & $23.5, \mathrm{CH}_{2}$ & $\begin{array}{l}1.65, \mathrm{~m} \\
1.94, \mathrm{~m}\end{array}$ & $33.8, \mathrm{CH}_{2}$ & $1.64, \mathrm{~m}$ & $23.7, \mathrm{CH}_{2}$ & $\begin{array}{l}1.73, \mathrm{~m} \\
1.85, \mathrm{~m}\end{array}$ \\
\hline 9 & $73.5, \mathrm{CH}_{2}$ & $3.85, \mathrm{~m}$ & $82.3, \mathrm{CH}$ & 3.86, ddd $(2.2,4.7,11.0)$ & $77.8, \mathrm{CH}$ & 4.14 , ddd $(2.1,4.3,12.6)$ & $77.3, \mathrm{CH}$ & $4.10, \mathrm{~m}$ \\
\hline 10 & $70.9, \mathrm{CH}$ & $3.52, \mathrm{dd}(5.6,11.5)$ & 73.6, CH & $3.64, \mathrm{~m}$ & $73.5, \mathrm{CH}$ & $3.68, \mathrm{dd}(5.6,11.5)$ & $73.2, \mathrm{CH}$ & $3.61, \mathrm{~m}$ \\
\hline 11 & $32.7, \mathrm{CH}_{2}$ & $\begin{array}{l}1.58, \mathrm{~m} \\
1.76, \mathrm{~m}\end{array}$ & $33.6, \mathrm{CH}_{2}$ & $1.59, \mathrm{~m}$ & 26.6, $\mathrm{CH}_{2}$ & $\begin{array}{l}1.41, \mathrm{~m} \\
1.54, \mathrm{~m}\end{array}$ & $34.0, \mathrm{CH}_{2}$ & $1.61, \mathrm{~m}$ \\
\hline 12 & 26.7, $\mathrm{CH}_{2}$ & $1.33, \mathrm{~m}$ & $26.5, \mathrm{CH}_{2}$ & $1.34, \mathrm{~m}$ & $30.4, \mathrm{CH}_{2}$ & $1.34, \mathrm{~m}$ & $28.1, \mathrm{CH}_{2}$ & $1.37, \mathrm{~m}$ \\
\hline 13 & $30.4, \mathrm{CH}_{2}$ & $1.33, \mathrm{~m}$ & $30.4, \mathrm{CH}_{2}$ & $1.34, \mathrm{~m}$ & $32.4, \mathrm{CH}_{2}$ & $1.86, \mathrm{~m}$ & $30.4, \mathrm{CH}_{2}$ & $1.34, \mathrm{~m}$ \\
\hline 14 & $33.0, \mathrm{CH}_{2}$ & $1.32, \mathrm{~m}$ & $33.0, \mathrm{CH}_{2}$ & $1.33, \mathrm{~m}$ & $33.0, \mathrm{CH}_{2}$ & $1.33, \mathrm{~m}$ & $30.5, \mathrm{CH}_{2}$ & $1.31, \mathrm{~m}$ \\
\hline 15 & 23.7, $\mathrm{CH}_{2}$ & $1.33, \mathrm{~m}$ & 23.7, $\mathrm{CH}_{2}$ & $1.30, \mathrm{~m}$ & 23.7, $\mathrm{CH}_{2}$ & $1.34, \mathrm{~m}$ & $26.8, \mathrm{CH}_{2}$ & $1.32, \mathrm{~m}$ \\
\hline 16 & $14.4, \mathrm{CH}_{3}$ & $0.91, \mathrm{t}(6.6)$ & $14.4, \mathrm{CH}_{3}$ & $0.91, \mathrm{t}(6.7)$ & $14.4, \mathrm{CH}_{3}$ & $0.91, \mathrm{t}(6.7)$ & $14.4, \mathrm{CH}_{3}$ & $0.90, \mathrm{t}(6.7)$ \\
\hline
\end{tabular}




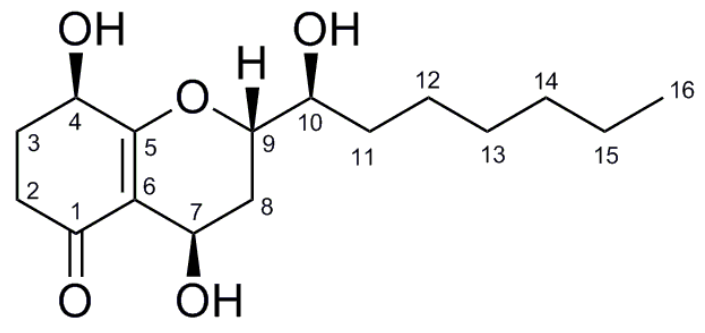

Figure 5.16: Structure of Koninginin D (5.9).

Koninginin D (5.9); $18.1 \mathrm{mg}$; light yellow solid; $[\alpha]_{\mathrm{D}} 116.6$ (c 0.8, MeOH); UV (MeOH)/nm $\lambda_{\max }$ ( $\log \varepsilon$ ) 260 (2.85); HRESIMS $m / z 299.1864[\mathrm{M}+\mathrm{H}]^{+}$(calculated for $\mathrm{C}_{16} \mathrm{H}_{27} \mathrm{O}_{5}, 299.1858$ ). ${ }^{1} \mathrm{H}$ and ${ }^{13} \mathrm{C}$ NMR data were consistent with published data (Dunlop et al. 1989) and are reported in table 5.5. The structure of koninginin $\mathrm{D}$ (5.9) can be found in figure 5.16.

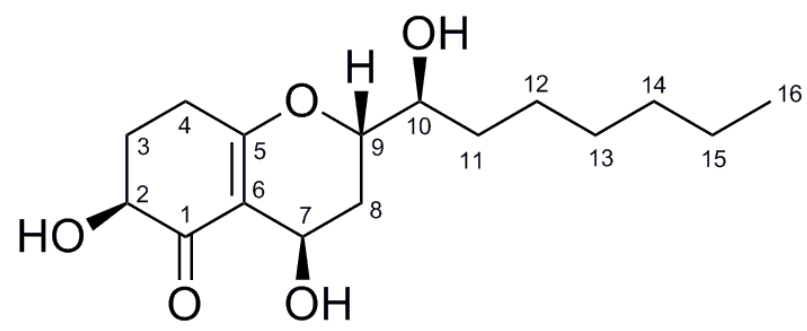

Figure 5.17: Structure of Koninginin F (5.10).

Koninginin F (5.10); $8.0 \mathrm{mg}$; light yellow solid; $[\alpha]_{\mathrm{D}} 122.2$ (c 0.4, MeOH); UV (MeOH)/nm $\lambda_{\max }$ ( $\log \varepsilon) 253$ (2.78); HRESIMS $m / z 299.1867[\mathrm{M}+\mathrm{H}]^{+}$(calculated for $\mathrm{C}_{16} \mathrm{H}_{27} \mathrm{O}_{5}, 299.1858$ ). ${ }^{1} \mathrm{H}$ and ${ }^{13} \mathrm{C}$ NMR data were consistent with published data (Liu and Wang. 2001) and are reported in table 5.5. The structure of koninginin F (5.10) can be found in figure 5.17. 
The polyketide derived koninginins characterized here (5.7-5.10) have been previously isolated from T. koningii and T. harzianum. Koninginin A (5.7) was previously isolated during this work from P. corylophilum; however, was first isolated from a $T$. koningii strain growing on a wilting plant (Cutler et al. 1989) and later from T. harzianum (Ghisalberti and Rowland 1993).

Koninginin A displayed weak inhibitory activity against the growth of etiolated wheat coleoptiles (Cutler et al. 1989), was inactive against both Gram-positive and negative strains examined (Cutler et al. 1991). Koninginin B (5.8) was originally isolated from the same $T$. koningii isolate as koninginin A (Cutler et al. 1991). It demonstrated slight antagonistic properties towards some Gram-positive bacteria including B. subtilis, B. cereus, and $M$. thermosphactum. However, koninginin B did not show any bioactivity against Gram-negative bacterial strains, such as E. coli (Cutler et al. 1991). At low concentrations $\left(\sim 10^{-5}\right.$ to $\left.10^{-6} \mathrm{M}\right)$ koninginin B stimulated plant growth in tomato seedlings (Vinale et al. 2006). Koninginin D (5.9) was isolated as the major component of a T. koningii soil isolate extract and inhibited the growth of various soil pathogens including Rhizoctonia solani, Phytophthora cinnamomi, Pythium middletonii, Fusarium oxysporum and Bipolaris sorokiniana (Dunlop et al. 1989). Koninginins D and $\mathrm{F}(\mathbf{5 . 1 0})$ are weakly antifungal against the fungus Gaeumannomyces graminis var tritici; a fungus that causes take-all in wheat and barley (Ghisalberti and Rowland 1993).

Koninginins are structurally similar to vitamin E, a known biological antioxidant that also possesses anti-inflammatory properties (Souza et al. 2008). The anti-inflammatory properties of koninginin $\mathrm{A}, \mathrm{E}$ and $\mathrm{F}$ were evaluated in response to pit viper venom. Koninginin $\mathrm{E}$ and $\mathrm{F}$ both exhibited anti-inflammatory properties whereas koninginin A did not (Souza et al. 2008). Due to 
the koninginins plant growth regulatory properties, unique structures and high number of chiral centers, total synthesis of a number of these natural products have been reported. A total synthesis for koninginin A was performed by Xu and Zhu (1995), Mori and Abe (1995), as well as Liu and Wang (2001). Liu and Wang (2000) describe the synthesis of koninginins B, D, E and F.

Samuels et al. (2006) conducted a phylogentic investigation of $T$. koningii that revealed three distinct evolutionary lineages. $T$. koningiopsis belonged to lineage 1 with $T$. koningii so it should not be surprising that multiple koninginins were isolated from this indoor derived T. koningiopsis strain. However, this may explain why other metabolites (i.e. 6-pentyl-alpha-pyrone) reported from closely related species were not isolated here. Previous reports of koninginins from $T$. koningii may actually have been from $T$. koningiopsis or vice versa as taxonomic revisions have complicated the literature on secondary metabolite profiles. T. koningiopsis is common in tropical America; however, has been reported from Canada, East Africa and Europe (Samuels et al. 2006).

\section{Secondary Metabolites from Trichroderma atroviride}




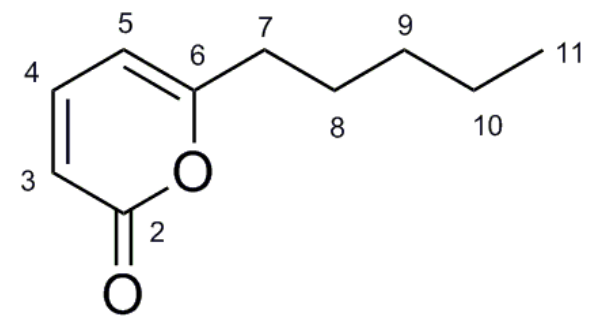

Figure 5.18: Structure of Pyrone 6- pentyl-2H-pyran-2-one (5.11).

Pyrone 6- pentyl-2H-pyran-2-one (5.11); $27.8 \mathrm{mg}$; dark brown oil; UV (MeOH)/nm $\lambda_{\max }(\log \varepsilon)$ 260 (4.52), 293 (4.60), 370 (4.45); HRESIMS m/z $167.1076[\mathrm{M}+\mathrm{H}]^{+}$(calculated for $\mathrm{C}_{10} \mathrm{H}_{15} \mathrm{O}_{2}$, 167.1072). ${ }^{1} \mathrm{H}$ data were consistent with published data (Colins and Halim 1972) and both ${ }^{13} \mathrm{C}$ and ${ }^{1} \mathrm{H}$ data are reported in table 5.6. The structure of compound $\mathbf{5 . 1 1}$ can be found in figure 5.18.

Table 5.6: ${ }^{1} \mathrm{H}(400 \mathrm{MHz})$ and ${ }^{13} \mathrm{C}$ NMR Data (100 MHz) pyrone 6- pentyl-2H-pyran-2-one (5.11) in $\mathrm{CD}_{3} \mathrm{OD}$.

\begin{tabular}{ccc}
\hline Position & $\delta_{\mathrm{C}, \text { type }}$ & $\delta_{\mathrm{H}}(J, \mathrm{~Hz})$ \\
\hline $\mathbf{2}$ & $165.9, \mathrm{C}$ & \\
$\mathbf{3}$ & $113.5, \mathrm{CH}$ & $6.15, \mathrm{~d}(9.4)$ \\
$\mathbf{4}$ & $146.0, \mathrm{CH}$ & $7.45 \mathrm{dd}(6.5,9.4)$ \\
$\mathbf{5}$ & $104.6, \mathrm{CH}$ & $6.19, \mathrm{~d}(6.5)$ \\
$\mathbf{6}$ & $168.1, \mathrm{C}$ & \\
$\mathbf{7}$ & $34.3, \mathrm{CH}_{2}$ & $2.51, \mathrm{t}(7.6)$ \\
$\mathbf{8}$ & $27.8, \mathrm{CH}_{2}$ & $1.66, \mathrm{~m}$ \\
$\mathbf{9}$ & $32.0, \mathrm{CH}_{2}$ & $1.33, \mathrm{~m}$ \\
$\mathbf{1 0}$ & $23.5, \mathrm{CH}_{2}$ & $1.35, \mathrm{~m}$ \\
$\mathbf{1 1}$ & $14.3, \mathrm{CH}_{3}$ & $0.91, \mathrm{t}(7.0)$ \\
\hline
\end{tabular}

The EtOAc extracts of T. atroviride had very few secondary metabolites in them by LC-UV-MS. Only pyrone 6- pentyl-2H-pyran-2-one (5.11) could be unambiguously characterized from this extract. Six of the nine Trichoderma cultures investigated here were identified by ITS sequence 
as $T$. atroviride and each of their filtrate extracts were the same by LC-UV-MS. This simple pyrone appears to be representative of the Trichoderma genus and was first reported from the fermentation broth of $T$. viride (Collins and Halim 1972). It has also been reported from $T$. harzianum (Ghisalberti and Rowland 1993) and T. koningii (Simon et al. 1988). Compound 5.11 has been extensively investigated for various applications as it is both antifungal and phytotoxic as well as being a food additive due to its characteristic coconut odor (Collins and Halim 1972; Reino et al. 2008). The addition of $0.3 \mathrm{mg} \mathrm{mL}^{-1}$ of compound $\mathbf{5 . 1 1}$ to agar medium inhibited the growth of Rhizoctonia solani and F. oxysporum by 69.6 and $31.7 \%$, respectively after 2 days. A concentration of $0.45 \mathrm{mg} \mathrm{mL}^{-1}$ completely inhibited the germination of Fusarium spores. The antifungal activity of $T$. harzianum has been strongly correlated with the production of this metabolite. The reduced form of 5.11, 6- pent-1-enyl-2H-pyrane-2-one (5.12), has also been reported from T. harzianum. Compound $\mathbf{5 . 1 2}$ elicited antimicrobial activity against a variety of Penicillium species, A. fumigatus, C. albicans and C. neoformans (Parker et al. 1997). This metabolite has been tentatively characterized here; however, insufficient amounts of the compound could be isolated to obtain a ${ }^{13} \mathrm{C}$ spectra as it co-eluted with $\mathbf{5 . 1 1}$ which was the major constituent of the extract. Only a weak ${ }^{1} \mathrm{H}$ spectra could be obtained that showed many of the same resonances as compound 5.11, see table 5.6. The ${ }^{1} \mathrm{H}$ spectra of $\mathbf{5 . 1 2}$ showed an additional doublet at $\delta 6.16(15.4 \mathrm{~Hz})$ and a doublet of triplets at $\delta 6.68(7.2$ and $15.4 \mathrm{~Hz})$ that would account of the addition of the trans double bond at C-7 and C-8. The coupling constants and chemicals shifts of these two additional proton resonances are consistent with those of a related compound, pyrone 6- hept-1-enyl-2H-pyrane-2-one (Zhang et al. 2007). The UV data were similar to those of $\mathbf{5 . 1 1}$ and the HRESIMS data corresponded to the correct molecular formula 
for the proposed compound, $\mathrm{C}_{10} \mathrm{H}_{14} \mathrm{O}_{2}$. This simple pentaketide was also identified by ${ }^{1} \mathrm{H}$ NMR, MS, UV and IR from T. viride by Moss et al. (1975).

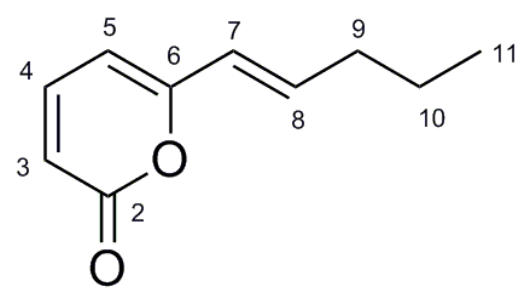

Figure 5.19: Structure of 6- pent-1-enyl-2H-pyrane-2-one (5.12) tentatively isolated from $T$. atroviride.

Pyrone 6- pent-1-enyl-2H-pyrane-2-one (5.12); $0.8 \mathrm{mg}$; light brown oil; UV (MeOH)/nm $\lambda_{\max }$ ( $\log \varepsilon) 370$ (4.45) 293 (4.60) 260 (4.52); HRESIMS m/z $165.0921[\mathrm{M}+\mathrm{H}]^{+}$(calculated for, $\left.\mathrm{C}_{10} \mathrm{H}_{15} \mathrm{O}_{2}, 165.0915\right) ;{ }^{1} \mathrm{H}$ NMR data were consistent with published data of the related pyrone , pyrone 6- hept-1-enyl-2H-pyrane-2-one (Zhang et al. 2007). The structure of compound $\mathbf{5 . 1 2}$ can be found in figure 5.19 . 


\section{EXPERIMENTAL}

\section{Trichoderma Cultures}

Nine Trichoderma strains isolated from Canadian buildings were obtained from Paracel Laboratories Ltd., Ottawa, Ontario (table 5.7). DNA was extracted from the mycelia using an UltraClean DNA Isolation Kit. ITS-1 and 4 sequences were amplified by PCR using the respective primers: 5'- TCCGTAGGTGAACCTGCGG-3' and 5'-TCCTCCGCTTATTGATA TGC-3') and sequencing was performed by Genome Quebec Innovation Centre, Montreal, Quebec. The sequenced PCR fragments were subsequently aligned using MAFFT and queried using the BLASTN algorithm against the NCBI nucleotide collection database. Five strains were tentatively identified as $T$. atroviride, two as T. koningiopsis, one as $T$. citrinoviride and $T$. harzianum (table 5.7).

Table 5.7: Indoor Trichoderma strains examined for metabolite production, location and fungal source.

\begin{tabular}{llll}
\hline Strain & Species & Location & Source \\
\hline DAOM 242931 & T. citrinoviride & Montreal, QC & Indoor air sample \\
DAOM 242933 & T. koningiopsis & Ottawa, ON & Indoor air sample \\
DAOM 242939 & T. koningiopsis & Ottawa, ON & Indoor air sample \\
DAOM 242934 & T. atroviride & Ottawa, ON & Indoor air sample \\
DAOM 242935 & T. atroviride & Ottawa, ON & Indoor air sample \\
DAOM 242937 & T. harzianum & Halifax, NS & Building materials \\
DAOM 242938 & T. atroviride & Sarnia, ON & Building materials \\
DAOM 242940 & T. atroviride & Montreal, QC & Indoor air sample \\
DAOM 242941 & T. atroviride & Sarnia, ON & Building materials \\
\hline
\end{tabular}




\section{Fermentation and Metabolite Screening}

All Trichoderma strains were aseptically transferred to sterile 2\% MEA plates and test tube slants. The slants and plates were left to incubate in the dark at $25^{\circ} \mathrm{C}$ for a period of $3-5$ days, sealed with parafilm and stored at $8^{\circ} \mathrm{C}$ until needed.

A slant of each Trichoderma strain was used to inoculate (5\%,v/v) twelve Roux bottles containing $200 \mathrm{~mL}$ of one of three different media: malt extract broth (ME; $\%$ malt extract), dextrose casein broth (DC; $4 \%$ glucose, $1 \%$ casein hydrolase) and glucose, yeast extract, malt extract, peptone, salts broth (GYM-salts; $2 \%$ glucose, $0.5 \%$ yeast extract, $0.5 \%$ malt extract, $0.5 \%$ peptone, $\left.0.25 \% \mathrm{~K}_{2} \mathrm{PO}_{4}, 0.2 \% \mathrm{MgSO}_{4} \cdot 7 \mathrm{H}_{2} \mathrm{O},\left(\mathrm{NH}_{4}\right)_{2} \mathrm{SO}_{4}\right)$. A Roux bottle containing each of the individual media was extracted at one week intervals for a period of four weeks ( 3 media $x 4$ weeks) to determine the optimal fermentation conditions for each indoor strain.

The liquid cultures were filtered (Whatman \#4) and the volume of the culture filtrate and $\mathrm{pH}$ were recorded. The culture filtrates were saturated with $\mathrm{NaCl}$, exhaustively extracted with EtOAc and filtered (Whatman \#1) through anhydrous sodium sulfate prior to being dried by rotary evaporation. The dried crude extracts were weighed, re-dissolved in a minimal amount of HPLC grade $\mathrm{MeOH}$, filtered through a $13 \mathrm{~mm}$ PTFE $0.2 \mu \mathrm{m}$ filter, and dried under a gentle stream of nitrogen gas.

Each extract was dissolved in $1 \mathrm{~mL}$ of HPLC grade methanol and analyzed by liquid chromatography-ultra violet-mass spectrometry (LC-UV-MS). This was achieved using a Waters 
2795 separations module, Waters 996 diode array detector, and Waters MicroMass Quattro LC mass spectrometer. Compounds were separated by a Phenomenex Kinetex $\mathrm{C}_{18}(100 \times 4.60 \mathrm{~mm}$, 2.6 $\mu \mathrm{m}, 100 \AA$ ) column (Torrance, California) using a mobile phase consisting of $\mathrm{ACN}-\mathrm{H}_{2} \mathrm{O}$ with FA; 0.1\%, (v/v). The solvent gradient was linear programmed from 5 to $100 \%$ ACN over 13 minutes with a flow rate of $1.0 \mathrm{~mL} \mathrm{~min}^{-1}$. Based on the LC-UV-MS data and extract masses, the optimal fermentation conditions for a representative strain of each Trichoderma species was determined. The chromatograms of each tentatively identified T. atroviride and T. koningiopsis strains were identical. The DC media was relatively unproductive for generating high yielding culture filtrate metabolite extracts. 2\% ME and GYMP-salts media generated similar LC-UV-MS chromatograms; however, the latter media produced greater extract masses.

\section{Metabolite Isolation}

Large scale fermentations were carried out with a single strain of T. citrinoviride (DAOM 242931), T. koningiopsis (DAOM 2422933) and T. atroviride (DAOM 2422934). The optimal fermentation conditions determined during the screening process were GYMP-salts media, $25^{\circ} \mathrm{C}$, stationary in the dark for two weeks for T. citrinoviride and four weeks for T. koningiopsis and T. atroviride. Ten $250 \mathrm{~mL}$ Erlenmeyer flasks containing $50 \mathrm{~mL}$ of sterile liquid media were inoculated $(5 \%, \mathrm{v} / \mathrm{v})$ and incubated using conditions elucidated until sufficient growth was observed ( $\sim 3$ days). Each of the ten starter cultures were macerated, individually transferred to Glaxo bottles containing $1 \mathrm{~L}$ of the same sterile media and left for two or four weeks under the same fermentation conditions described above. The culture filtrate was extracted as described above. 


\section{Isolation of $T$. citrinoviride Metabolites}

The crude EtOAc culture filtrate extract $(2.1 \mathrm{~g})$ was fractionated by flash column chromatography with a silica gel $(40-63 \mu \mathrm{m})$ column using a step gradient elution with hexanesEtOAc (0-100\%) in 10\% increments. Two bright yellow bands eluted in 50\% EtOAc and were collected independently (Fractions 7 and 8). A third of fraction 7 (105 mg) was subjected RP semi-preparative HPLC using a Phenomenex Luna $\mathrm{C}_{18}(250 \times 10.00 \mathrm{~mm}, 5 \mu \mathrm{m}, 100 \AA)$ column. Fraction 7 was fractionated by a linear gradient that was programmed from $25-100 \% \mathrm{ACN}-\mathrm{H}_{2} \mathrm{O}$ over 19 minutes with a flow rate of $4 \mathrm{ml} \mathrm{min}^{-1}$. Fraction 7 yielded $R$-vertinolide (5.1, $6.4 \mathrm{mg}$ ), spirosorbicillinol C (5.4, 5.0 $\mathrm{mg})$ and bisvertinol (5.6, $7.0 \mathrm{mg})$. Fraction $8(85 \mathrm{mg})$ was separated by a similar HPLC program, 30-100\% ACN-H $\mathrm{A}_{2} \mathrm{O}$ over 19 minutes, that yielded spirosorbicillinol A (5.2, $9.7 \mathrm{mg})$, spirosorbicillinol B (5.3, $4.7 \mathrm{mg})$ and trichotetronine $(\mathbf{5 . 5}, 11.1 \mathrm{mg})$.

\section{Isolation of $T$. koningiopsis Metabolites}

The crude EtOAc culture filtrate extract (2.3 g) was subjected to vacuum liquid chromatography on a short silica gel column using a step gradient elution with hexanes-EtOAc $(0-100 \%)$ followed by EtOAc-MeOH (0-100\%) in 20\% increments yielding 10 fractions. Fraction 2 (105.4 mg) eluted in 20\% hexanes-EtOAc and was further fractionated by RP semi-preparative HPLC using a Phenomenex Luna $\mathrm{C}_{18}(250 \times 10.00 \mathrm{~mm}, 5 \mu \mathrm{m}, 100 \AA)$ column. The linear gradient was programmed from $10-80 \% \mathrm{ACN}-\mathrm{H}_{2} \mathrm{O}$ over 25 minutes with a flow rate of $4 \mathrm{ml} \mathrm{min}{ }^{-1}$. Fraction 2 yielded koninginin A (5.7, $17.9 \mathrm{mg})$. Fraction 5 (487.3 mg) eluted in 70-90\% EtOAc and was fractionated by flash column chromatography utilizing a silica gel column and $\mathrm{MeOH}-\mathrm{CHCl}_{3}$ 
gradient that increased from $0-20 \% \mathrm{MeOH}-\mathrm{CHCl}_{3}$ in $2 \%$ increments yielding ten fractions.

Fraction $5.3(99.6 \mathrm{mg})$ eluted in 3\% MeOH and yielded koninginin $\mathrm{B}(\mathbf{5 . 8}, 21.9 \mathrm{mg})$ after two successive HPLC purifications. Fractions 5.4 to $5.7(324.4 \mathrm{mg})$ eluted in 5-9\% $\mathrm{MeOH}-\mathrm{CHCl}_{3}$, were combined and fractionated by flash column chromatography using a silica gel column and $\mathrm{MeOH}-\mathrm{CHCl}_{3}$ gradient from $0-12 \% \mathrm{MeOH}$ in $3 \%$ increments. This afforded a fraction (107.9 $\mathrm{mg}$ ) that eluted in 3\% MeOH that provided koninginin $\mathrm{D}(\mathbf{5 . 9}, 18.1 \mathrm{mg})$ and koninginin $\mathrm{F}$ (5.10, $8.0 \mathrm{mg}$ ) after HPLC purification using the same conditions described for $\mathbf{5 . 7}$.

\section{Isolation of T. atroviride Metabolites}

The crude EtOAc culture filtrate extract (1.4 g) was fractionated by flash column chromatography with a silica gel $(40-63 \mu \mathrm{m})$ using a $\mathrm{MeOH}-\mathrm{CHCl}_{3}$ gradient that increased from

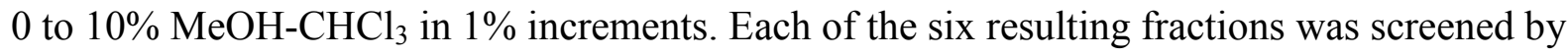
LC-UV-MS as previously described. Each of their chromatograms was similar to one another and of the crude extract. A column fraction with a mass (120.4 mg) suitable for further purification was subjected to RP semi-preparative HPLC using a Phenomenex Luna $\mathrm{C}_{18}(250 \mathrm{x}$ $10.00 \mathrm{~mm}, 5 \mu \mathrm{m}, 100 \AA$ ) column and linear gradient was programmed from $20-100 \% \mathrm{ACN}-\mathrm{H}_{2} \mathrm{O}$

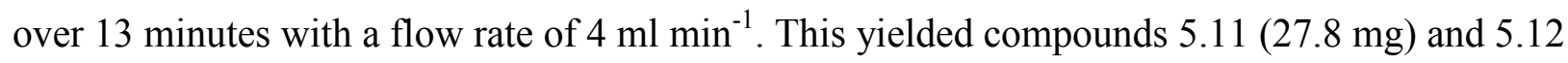
$(0.8 \mathrm{mg})$.

\section{General Experimental Procedures}

Metabolite NMR spectra were obtained on a Bruker Avance 400 Spectrometer (Milton, On) at $400.13\left({ }^{1} \mathrm{H}\right)$ and $100 \mathrm{MHz}\left({ }^{13} \mathrm{C}\right)$ using a $5 \mathrm{~mm}$ auto-tuning broadband probe with a Z-gradient. 
Secondary metabolites were dissolved in $\mathrm{CD}_{3} \mathrm{CN}(\delta \mathrm{H} 1.94$ and $\delta \mathrm{C} 118.7 / 1.39)$, or $\mathrm{CD}_{3} \mathrm{OD}(\delta \mathrm{H}$ 3.30 and $\delta \mathrm{C} 49.0$ ) (CDN Isotopes, Point Claire, QC) and were referenced to the solvent peak.

Chemicals shifts were assigned based on ${ }^{1} \mathrm{H},{ }^{13} \mathrm{C}, \mathrm{COSY}, \mathrm{HSQC}, \mathrm{HMBC}$, and DEPT-135 spectra utilizing standard Bruker pulse sequences. High resolution mass spectrometry data were acquired on a system equipped with an Agilent 1100 binary pump connected to an Agilent G1969A LC/MSD TOF HRMS. Compounds were separated on a Phenomenex Zorbax $\mathrm{C}_{18}(50$ x $3.0 \mathrm{~mm}$, $1.8 \mu \mathrm{m})$ column using a mobile phase consisting of $\mathrm{ACN}-\mathrm{H}_{2} \mathrm{O}$ with formic acid $(0.1 \%$, v:v). The gradient was linear programmed from 10 to $95 \%$ ACN over 4 minutes at a flow rate of $1 \mathrm{~mL}$ $\min ^{-1}$. The MS was operated in both positive and negative ion mode. UV spectra of all major metabolites were obtained using a Varian Cary 3 UV-visible spectrophotometer scanning from 190- $800 \mathrm{~nm}$. Optical rotations were determined using an Autopol IV polarimeter. 


\section{CHAPTER VI}

\section{GENERAL DISCUSSION}


There is now a lot known about the fungal metabolites that are produced by fungi that commonly occur in damp buildings. This has been achieved by the acquisition of more reliable information regarding the fungi common on damp building materials. As previously described, the fungal communities that occur on damp building materials are primarily a consequence of how wet the material gets, the period of time it remains wet and the chemistry of the material. However, since similar building materials are utilized in developed countries, the frequency of the most common species appears similar in temperate zones. This assertion is supported by higher resolution information obtained from next generation sequencing studies (Amemd et al. 2010; Dannemiller et al. 2014). Based on traditional culture techniques the fungi associated with particular damp building materials have been summarized for Europe (Andersen et al. 2011) as well as Canada and the USA (Miller et al. 2008).

As different building materials afford their own unique combination of nutrients, chemistry, available water and other physical factors, it should be no surprise that specific fungi are associated with different damp materials. Some of the most commonly used building materials include insulation, gypsum wallboard, cellulose-based products such as wood, manufactured food, ceiling tiles and textiles. Many Penicillium species can be found on wet insulation due to their ability to tolerate formaldehyde. Cladosporium species also appear to be fairly common on insulation. However, as many of these species are present in outdoor air and are common on surfaces, finding these species by traditional culture methods does not necessarily indicate their growth on the substrate indoors (AIHA 2008). 
Three factors that directly affect the mycoflora on gypsum wallboard are water activity, nutrients and concentration of salts, particularly calcium salts. C. globosum, S. chartarum, Memnoniella echinata, A. versicolor, A. sydowii, Paecilomyces variotii, C. sphaerospermum, P. chrysogenum, P. commune and E. herbariorum are all common on gypsum wallboard. These species have been recovered from alkaline soils and have a high tolerance for calcium salts.

Some of the fungi found growing on wet solid wood are also found on various manufactured wood products. Variation of fungi growing on wood can be caused by the type of wood (soft or hard) used as each will require different fungal biochemistry (i.e. secretion of enzymes). Wood based products may also vary from different manufacturers. Textiles are much more difficult to generalize compared to wood as they can be derived from multiple components. Molds found growing on wool for example would be required to degrade keratin. However, fungi commonly found growing on textiles are also prevalent on gypsum board, wood and wood products. Some dominant species on wood and wood based products include C. globosum, S. chartarum, $P$. chrysogenum, P. brevicompactum, C. sphaerospermum, A. versicolor, T. harzianum, Paecilomyces variotii and Ulocladium chartarum (AIHA 2008).

Common food-borne fungi typically produce secondary metabolites optimally when the water activity of the substrate is high, near 1.0 , and at mesophilic temperatures, $20-25^{\circ} \mathrm{C}$ (Frisvad and Thrane 1995). The majority of fungal metabolite production investigations on damp building materials have been performed when the water activity is between $0.95-0.99$ at $25^{\circ} \mathrm{C}$ (Nielsen 2003). The influence of relative humidity and temperature on growth and secondary metabolite production of common indoor fungi for variety of building materials has been studied. The lower 
water activity limit for fungal growth on wood and wood based products was 0.78 at $20-25^{\circ} \mathrm{C}$. This increased to 0.90 at $5{ }^{\circ} \mathrm{C}$. At lower water activities, some of the more xerophilic Penicillium, Aspergillus and Eurotium species dominated. Their production of secondary metabolites was drastically reduced on building materials at lower water activities in comparison to when the water activity was $>0.95$. The exception was a xerophilic Eurotium species (Nielsen et al. 2004). The production of walleminone by the xerophile $W$. sebi increased as the amount of solute was added to the liquid medium lowering the water activity (Frank et al. 1999; Wood et al. 1990). Indoor strains of $W$. sebi studied here would not grow let alone produce metabolites on high water activity media. Similar observations were made with P. corylophilum. Appreciable metabolite amounts were not generated from this moderately xerophilic Penicillium species when using $2 \%$ malt exact or Czapek Dox broth. It was only when it was grown using $15 \%$ yeast extract, sucrose liquid medium when this was achieved. Induction of metabolite production by low water activity conditions by xerophilic fungi was also observed by Sepcic et al. (2011). This indicates that some xerophilic fungi commonly found in damp buildings are capable of producing secondary metabolites when low water activity would appear to generate unfavorable conditions.

Many of the fungi commonly that have been commonly identified growing on damp building materials have been investigated for both secondary metabolites and human antigens due their deleterious impact on human health. The number of sensitized, atopic patient sera that respond to a specific mold allergen typically correlates with the frequency that those species are found in damp buildings in Canada and USA. The Fleming clade of $P$. chrysogenum, now called $P$. rubens is the most common species of Penicillium on damp building materials (Miller et al. 
2008). For this species, just over $50 \%$ of the patient sera in the Miller lab collection reacted to the allergen (Wilson et al. 2009). For another very common fungus, A. versicolor, $20 \%$ reacted and for the extensively studied $S$. chartarum sensu lato, $10 \%$ of the patient sera responded to its antigen (Xu et al. 2007). Human antigens have recently been identified for two additional prevalent indoor fungi that were examined in my investigation of secondary metabolites. Approximately $50 \%$ of the sera tested responded to a C. globosum chitosanase (Provost et al. 2013) and for the largely ignored $W$. sebi, 36\% responded to a cellulase (Desroches et al. 2014). C. globosum is reliably known to be a dominant fungi found on damp building materials (Andersen et al. 2011; Miller et al. 2008). However, the prevalence of antibodies responding to $W$. sebi indicates it is more common in the built environment than previously thought. $P$. corylophilum is known to cause allergic responses in humans (Unoura et al. 2011); however, the allergen has not been reported. Due to limited reports of its secondary metabolites and suspected prevalence indoors $P$. corylophilum was studied.

The most common species of Penicillium found on damp materials in the USA and Canada is the Fleming clade of $P$. chrysogenum, now called $P$. rubens. Indoor strains of this fungus make penicillin G, roquefortine C, meleagrin and xanthocillin X (De La Campa et al. 2007; Houbrachen et al. 2011). P. chrysogenum produces the same metabolites as $P$. rubens with the addition of secalonic acid D and F and a lumpidin-related compound (Houbrachen et al. 2011). Metabolites from Canadian strains of another common Penicillium species, $P$. brevicompactum, include brevianamide, mycophenolic acid (Rand et al. 2005), asperphenamate and a tanzawaic acid (Nielsen et al. 1999; 2004). An indoor air isolate of P. crustosum from Denmark produced roquefortine $\mathrm{C}$, penitrem A, viridicatols, terrestic acid and andrastin A (Sonjak et al. 2005). The 
mycotoxin citrinin, produced by many Penicillium species including $P$. citrinum, was detected in three Finish indoor samples (Tuomi et al. 2000).

One of the most common species found growing on water-damaged building materials, $A$. versicolor, produces the aflatoxin precursor sterigmatocystin and 5-methoxysterigmatocystin. These compounds have been directly detected on water-damaged building materials (Bloom et al. 2007; 2009; Nielsen et al. 1999; 2004; Tuomi et al. 2000) air samples and dust collected from carpets and floors (Bloom et al. 2007; 2009; Engelhardt et al. 2002).

The isoquinoline alkaloids TMC-120 A-C, their derivatives and a drimane sesquiterpene were isolated from indoor strains designated Aspergillus ustus sensu latto. However, this species was revised recently and strains common in the built environment are A. insuetus and A. calidoustus (Slack et al. 2009). TMC-120 A has been detected on building materials contaminated with this species. A. ochraceus is also common indoors; however, is more often associated with concrete and flooring (Andersen et al. 2011). This species produces a variety of metabolites including the nephrotoxic ochratoxin A, penicillic acid, xanthomegnin, viomellein and vioxanthin (Frisvad and Thane 2002). Ochratoxin A was originally reported indoors from moldy pet food debris in house dust (Richard et al. 1999) and has since been detected in from damp building materials (Taübel et al. 2011). Aflatoxin B1 and B2 were also identified from indoor air, dust and biomass samples, but as was the case for ochratoxin A, the producing species could not be identified (Polizze et al. 2009). Aflatoxin B1 was detected from indoor air samples collected from hospitals that were positive for A. flavus (Bloom et al. 2009). 
In North American buildings, the dominant species of Eurotium, an Aspergillus teleomorph, are E. amstelodomi and E. herbariorum where E. rubrum is less common. Neoechinulin A and B, epiheveadride, flavoglaucin, auroglaucin and isotetrahydroauroglaucin are the major metabolites produced by most Eurotium species. E. rubrum produced all of these compounds but epiheveadride was detected only in minor amounts. The major metabolite of E. herbariorum is cladosporin that was not isolated from E. amstelodomi or E. rubrum. Strains from the built environment in Canada and the USA accumulate a number of compounds in the mycelium including echinulin which has been detected on building materials (Nielsen et al. 2004; Slack et al. 2009).

Damp building strains of the two species of Stachybotrys that occur in the US and Canada make many biologically active compounds (Miller et al. 2003). S. chartarum sensu latto was separated into two distinct chemotypes and related species, S. chlorohalonata (Andersen et al. 2002; 2003). S. chartarum chemotype $\mathrm{S}$ produces the macrocyclic trichothecene satratoxins $(\mathrm{G}$ and $\mathrm{H})$ and roridin E, which has been directly detected from indoor air and dust samples (Polizzi et al. 2009). Chemotype A produces atranones A, B, F and E, their precursors and dolabellanes (Andersen et al. 2002; Nielsen et al. 1998; Rand et al. 2006; Vesper et al. 2002). S. chlorohalonata could be differentiated based on metabolite profiles from chemotype S (Andersen et al. 2003). All three chemically distinct species produced the simple trichothecenes trichodermin and trichodermol, precursors to the macrocyclic trichothecenes. The dominant metabolites produced by both $S$. chartarum chemotypes and S. chlorohalonata were the immunosuppressant spirocyclic drimanes and their precursors, however, S. chlorohalonata was shown to generally produce less (Andersen et al. 2002; Nielsen 2003). While all Stachybotrys isolates investigated produced trichothecenes, 
approximately $40 \%$ synthesized the much more toxic macrocyclic trichothecenes (Andersen et al. 2002). This is a pertinent example of metabolite profiles being utilized to distinguish between two morphologically similar fungi.

Memnoniella echinata is closely related to S. chartarum, produces many of the same toxins and is found on similar building materials (Jarvis et al. 1998). It produces the simple trichothecenes trichodermin and trichodermol, xanthone and spirocyclic drimanes different than those from $S$. chartarum. However, M. echinata indoor isolates primarily produce several griseofulvins including dechlorogriseofulvin, and epi-dechlorogriseofulvin (Jarvis et al. 1996; 1998; Nielsen et al. 1998). Interestingly, this was the first example of a griseofulvin producing species outside the genus Penicillium (Jarvis et al. 1996). More recent phylogenetic analysis could not differentiate Memnoniella from Stachybotrys. The current correct name is Stachybotrys echinata (Samson et al. 2010).

Despite being one of the most common fungi found on damp building materials (Andersen et al. 2011; Miller et al. 2008) and a known producer of toxic secondary metabolites, surprisingly, Chaetomium globosum has received much less attention compared to other fungi common in the built environment such as $S$. chartarum. Strains of this fungus studied here produced primarily chaetoglobosin $\mathrm{A}, \mathrm{C}$ and $\mathrm{F}$, chaetomugilin $\mathrm{D}$, chaetoviridin $\mathrm{A}$ which all have potent bioactivities (McMullin et al. 2013). Other chaetoglobosins and azaphilones are also produced in minor amounts (McMullin et al. 2013). Some Chaetomium species are capable of producing sterigmatocystin; however, none of these species has been identified indoors. Chaetoglobosins A 
and C (Fogel et al. 2007; Nielsen et al. 1999; Täubel et al. 2011) and chetomin (Vishwanath et al. 2009) have all been reported from moldy building materials.

Metabolites produced by $P$. corylophilum isolates investigated here produced the eremophilane sesquiterpene phomenone, the meroterpenoids andrastin A and citreohybridonol, koninginins A, E and G, and a number of new to science metabolites (McMullin et al. 2014). The new $P$. corylphilum metabolites include four isochromans and three alpha pyrones (McMullin et al. 2014). Of the characterized metabolites, only phomenone was reported in the literature to be produced by $P$. corylophilum making these first reports of alpha pyrones, meroterpenoids from this surprisingly common indoor fungus. More surprisingly, this was the first observation of koninginins outside the genus Trichoderma.

A number of species of Trichoderma have been reported from damp building materials including T. longibrachiatum T. citrinoviride, T. harzianum, T. atroviride, T. viride, T. harmatum and T. koningii (Andersen et al. 2011; Lubeck et al. 2000). The simple trichothecenes trichodermin and or harzianum A were originally reported from $T$. viride and T. harzianum. However, these producing strains were later shown to be T. brevicompactum which is not found in the built envronment. Isolates of $T$. viride, $T$. harzianum, $T$. atroviride, $T$. longibrachiatum and $T$. citrinoviride from European buildings did not produce either trichothecene (Nielsen et al. 2005). Canadian strains of T. koningiopsis and T. atroviride from the Canadian buildings produced koninginins $\mathrm{A}, \mathrm{B}, \mathrm{D}$ and $\mathrm{F}$ and the simple alpha pyrone, 6- pentyl-2H-pyran-2-one, respectively. A T. harzianum strain produced the same metabolites as the T. atroviride strains investigated here. The production of low molecular weight pyrones and lactones is characteristic of this genus 
(Reino et al. 2008). An isolate of T. citrinoviride produced various sorbicillin-derived compounds including the $(R)$ isomer of vertinolide, spirosorbicillinol A-C, bisvertinol, and trichotetronine. From the related species, T. longibrachiatum, one 11- and eight 20- residue new cytotoxic peptaibols named trilongins were identified from a Finish indoor isolate (Mikkola et al. 2012).

The largely ignored xerophilic fungus Wallemia sebi is now known to be common in settled house dust in both North America and Europe. A strain recovered from dust in a school vent in Canada produced the sesquiterpene walleminone, a new alkaloid wallimidione, tryptophol and various other amino acid derived small compounds (Desroches et al. 2014).

To determine the impact fungi found in damp buildings have on human health, proper taxonomic identifications are required. This allows the real biodiversity and health hazards of the dominant or pertinent fungi to be investigated. Additionally, depositing strains in recognized culture collections allowing them to be studied further is critical to future studies. All indoor derived strains of C. globosum, P. corylophilum, W. sebi and Trichoderma species investigated here were obtained from recognized culture collections or have been deposited with the Canadian National Mycological Herbarium in Ottawa, Ontario.

I found that much of the existing literature concerning the secondary metabolites produced by fungi I studied had suffered from doubtful identifications, and in some cases, poor chemical analysis. The identification of fungi based on morphology has been shown to have multiple restrictions. Using molecular methods to examine gene sequences may yield species segregation 
of morphologically similar fungi (Samson et al. 2010). This can be exemplified by the $P$. roqueforti complex and related species examined in the Miller lab (Nielsen et al. 2006). In conjunction with morphological characteristics and gene sequences, secondary metabolite profiles can aid in the identification of fungi as the set of metabolites for a given species are typically consistently produced (Larsen et al. 2005). Polyphasic examinations of fungi have resulted in the taxonomic revisions of many fungal genera. Studying deposited Trichoderma strains from the indoor environment and employing a polyphasic approach demonstrated that trichothecene production within the genus appears to be restricted to the T. brevicompactum clade (Degenkolb et al. 2008; Nielsen et al. 2005). Resolving this incorrect taxonomy indicated that Trichoderma species commonly found indoors do not produce toxic trichothecenes.

All of the metabolites reported herein were purified from culture filtrate extracts and unambiguously structurally characterized by mass spectrometry, nuclear magnetic resonance, chemical derivatization and other spectroscopic methods. As the metabolites identified here all came from properly identified, deposited strains, this investigation of fungal secondary metabolites can aid future taxonomic studies of these or related species. Accurate descriptions of the metabolites profiles were made from spectroscopic data opposed to identifications based on chromatographic peaks and/or fragmentation patterns. Nielsen et al. (1999) studied the metabolite profiles of indoor C. globosum isolates identifying chaetoglobosin A and C as well as multiple unidentified compounds using HPLC-DAD. Comparison of the chromatographic and spectroscopic data for chaetoviridin A isolated during this work identifies it in the C. globosum extracts investigated by Nielsen et al. (1999). Purifying non-trivial amounts, a couple mg, of fully characterized metabolites from properly identified isolates aids in deconvoluting existing 
secondary metabolite literature, provides standards that can be used to quantify metabolite production or in toxicological experiments.

All fungi studied here were screened for metabolite production using a variety of different liquid media. Growing fungi on agar media for the purpose of metabolite screening has previously been demonstrated to underestimate their toxigenic potential (Nielsen et al. 2006). Large-scale fermentations with a variety of media have resulted in the characterization of unexpected metabolites from even very well studied fungi. This was apparent in P. corylophilum where alpha pyrones, meroterpenoids, and koninginins were identified for the first time from this species which included the characterizing seven new metabolites. Finding koninginins would probably have been as surprising as isolating griseofulvins from M. echinata. Koninginins and griseofulvins were reported for the first time from indoor strains when they had previously only been observed in the Trichoderma and Penicillum genera, respectively. This works has resulted in the discovery of at least one new metabolite from each of the four fungal genera studied. Three new azaphilones were isolated from C. globosum, an unusual alkaloid was identified from $W$. sebi, an unreported isomer of vertinolide was characterized from T. citrinoviride as well as the four new isochromans and $3 \alpha$-pyrones from $P$. corylophilum mentioned above. This demonstrates the use of different media and large-scale fermentations to discover new compounds, even from fungi that have been extensively studied.

A limited number of investigations of have demonstrated that exposures to secondary metabolites from fungi found growing in damp buildings cause various inflammatory responses. At the actual doses, $\mathrm{nM}$, that could be experienced by the human lung from metabolite exposures 
in a damp building with mold damage, many of these compounds, including mold specific glucan, modulate a variety of genes and chemokine responses. These responses are associated with asthma pathways and these observations have been made in vivo, in primary cells as well as relevant immortalized cell lines (Rand et al. 2011; 2013). Responses include dose, time and compound dependent transcription and expression of the genes associated with acute pulmonary inflammation. These alterations were especially apparent in alveolar marcophages where the induction of three chemokines, including tumour necrosis factor alpha (TNF- $\alpha$ ), involved in lung inflammation was observed by immuno-histochemistry (Rand et al. 2011). These genetic alterations are also accompanied by histological disruptions. These inflammatory responses provide a tangible etiology for some of the adverse health effects reported from individuals occupying damp, moldy buildings including non-atopic asthma (Akpina et al. 2013; Miller et al. 2010; Rand et al. 2010; 2011; 2011; 2013).

Investigations of the effects of metabolites from indoor fungi have on relevant endpoints includes studies on the spores of S. chartarum chemotypes that either produce macrocyclic trichothecenes or atranones (Flemming et al. 2003); brevianamide A and mycolpheolic acid ( $P$. brevicompactum), roquefortine C (P. rubens) (Rand et al. 2005); atranones A and C (S. chartarum) (Rand et al. 2006); sterigmatocystin (A. versicolor), TMC-120 A (A. ustus sensu latto), neoechinulin A and B (E. amstelodami, E. herbariorum and E. rubrum) cladosporin from E. herbariorum (Miller et al. 2010; Rand et al. 2011). In all cases, inflammatory responses were observed in response to metabolite exposures that could be experienced in a building with mold damage. 
The primary focus of this study was to elucidate the dominant metabolites from some prominent indoor fungi to determine how these chemicals alter biochemically relevant human endpoints. To conduct toxicological research aimed at elucidating the inflammatory properties of metabolites produced by common indoor fungi, first requires generation of very pure compounds. To continue these investigations, chaetoglobosin A, chaetomugilin D (C. globosum); phomenone, andrastin A (P. corylophilum), walleminone and tryptophol (W. sebi), koninginin A ( $T$. koningiopsis and $P$. corylophilum) and trichotetronine (T. citrinoviride) are currently being investigated for their inflammatory effects. Preliminary data have been obtained using a relevant mouse alveolar macrophage cell line, RAW 264.7 (Rand et al. 2013). Alveolar macrophages were utilized as they are considered defense cells of the respiratory system and are responsible for acute inflammation by releasing various chemokines into the lung (Rand et al. 2011). Considering the mechanistic importance of the chemokine TNF-a, up regulation was observed at $10^{-8} \mathrm{M}$ for both chaetoglobosin $\mathrm{A}$ and chaetomugilin $\mathrm{D}$, and $10^{-7} \mathrm{M}$ in the case of andrastin $\mathrm{A}$ and walleminone (Rand, personal communication). Information from these experiments will contribute to a more comprehensive understanding of the mechanism for non-atopic asthma and other adverse health effects associated with exposure to mold and dampness indoors (Rand et al. 2013). 


\section{LITERATURE CITED}

Abdel-Lareff A, Fisch A, Wright A (2009) Trichopyrone and other constituents from the marine sponge derived fungus Trichoderma sp. Zeiterschrift fur Naturforschung C, 64: 186-192.

Abe N, Arakawa T, Yamamoto K, Hirota A (2002) Biosynthesis of bisorbicillinoid in Trichoderma sp. USF-2690; evidence for the biosynthetic pathway, via sorbicillinol, of sorbicillin, bisorbicillinol, bisorbibutenolide, and bisorbicillinolide. Bioscience, Biotechnology and Biochemistry, 66: 2090-2099.

Abe N, Murata T, Hirota A (1998) Novel oxidized sorbicillin dimers with 1, 1 diphenyl-2picrylhydrazl-radical scavenging activity from fungus. Bioscience, Biotechnology and Biochemistry, 62: 2120-2126.

Abe N, Sugimoto O, Arakawa T, Tanji K, Hirota A (2001) Sorbicillinol, a key intermediate of bisorbicillinoid biosynthesis in Trichoderma sp. USF-2690. Bioscience, Biotechnology and Biochemistry, 65: 2271-2279.

Adams R, Miletto M, Taylor J, Bruns T (2013) Dispersal in microbes: fungi in indoor air are dominated by outdoor air and show dispersal limitation at short distances. The ISME journal, 7: $1262-73$.

Akpinar-Elci M, White S, Siegel P, Park J, Visotcky A, Kreiss K, Cox-Ganser J (2013) Markers of upper airway inflammation associated with microbial exposure and symptoms in occupants of a water-damaged building. American Journal of Industrial Medicine, 56: 522-530.

Amemiya Y, Kondo A, Hirano K, Hirukawa T, Kato T (1994) Antifungal substances produced by Chaetomium globosum. The Technical Bulletin of Faculty of the Horticulture ,Chiba Univesity, 48: 13-18.

Amend A, Seifert K, Samson R, Bruns T (2010) Indoor fungal composition is geographically patterned and more diverse in temperate zones than in the tropics. Proceedings of the National Academy of Science, 107: 13748-13753.

American Industrial Hygiene Association (2008) Position statement on mold and dampness in the built environment. AIHA, Fairfax, VA.

American Industrial Hygiene Association (2013) Position statement on mold and dampness in the built environment. AIHA, Fairfax, VA. 
Andersen B, Frisvad J, Søndergaard I, Rasmussen I, Larsen L (2011) Associations between fungal species and water-damaged building materials. Applied Environmental Microbiology, 77: 4180-4188.

Andersen B, Nielsen, KF, Jarvis BB (2002) Characterization of Stachybotrys from waterdamaged buildings based on morphology, growth and metabolite production. Mycologia, 94: 392-403.

Andersen B, Nielsen KF, Thrane U, Cruse M, Taylor J, Jarvis BB (2003) Stachybotrys chlorohalonata, a new species from waterdamaged buildings. Mycologia 95:1228-1237.

Andrade R, Ayer A, Latchezar S (1997) The Metabolites of Trichoderma longibrachiatum. III. Two New Tetronic Acids: 5-Hydroxyvertinolide and Bislongiquinolide. Australian Journal of Chemistry, 50: 255-258.

Andrade R, Ayer W, Mebe P (1992) The metabolites of Trichoderma longibrachiatum. Part 1. Isolation of the metabolites and the structure of trichodimerol. Canadian Journal of Chemistry, 70: $2526-2535$.

Andrade R, Ayer W, Trifonov L (1996) The metabolites of Trichoderma longibrachiatum. Part II. The structures of trichodermolide and sorbiquinol. Canadian Journal of Chemistry, 74: 371379.

Arai N, Shimo K, Tomoda H, Tabata N, Yang D, Masuma R, Kawakubo T, Omura S (1995) Isochromophilones III-VI, inhibitors of Acyl-CoA:cholesterol acyltransferase produced by Penicillium multicolor FO-3216. Journal of Antibiotics, 48: 696-702.

Asgari B and Zare R (2011) The genus Chaetomium in Iran, a phylogenetic study including six new species. Mycologia, 103: 863-882.

Badar Y, Lockley W, Toube T, Weedon B, Guy-Valadon L (1972) Natural and synthetic pyrrol2-ylpolyenes. Journal of the Chemical Society, Perkin Transactions 1, 13: 1416-1424.

Bashyal B, Wijeratne E, Faeth S, Gunatilaka A (2005) Globosumones A-C, cytotoxic orsellinic acid esters from the Sonoran desert endophytic fungus Chaetomium globosum. Journal of Natural Products, 68: 724-728.

Betina V (1995) Differentiation and secondary metabolism in some prokaryotes and fungi. Foliar Microbiology, 40: 51-67.

Betina V, Micekova D, Memec P (1972) Antimicrobial properties of cytochalasins and their alteration of fungal morphology. Journal of General Microbiology, 71: 343-349.

Binder M and Tamm C (1973) The cytochalasans: a new class of biological active microbial metabolites. Angewandte Chemie Intenational, 12: 370-380. 
Bissett J (1987) Fungi associated with urea-formaldehyde foam insulation in Canada. Mycopathologia, 99:47-56.

Blackwell M (2011) The Fungi: 1, 2, 3...5.1 million species? American Journal of Botany. 98: 426-438.

Bloom E, Bal K, Nyman E, Must A, Larsson L (2007) Mass spectrometry based strategy for the direct detection and quantification of some mycotoxins produced by Stachybotrys and Aspergillus spp. in indoor environments. Applied Environmental Microbiology, 73: 4211-4217.

Bloom E, Nyman E, Must A, Pehrson C, Larsson L (2009) Mold and mycotoxins in indoor environments-A survey in water damaged buildings. Journal of Occupation and Environmental Hygiene, 6:671-678.

Bok G, Hallenberg N, Aberg O (2009) Mass occurrence of Penicillium corylophilum in crawl spaces, south Sweden. Building and Environment, 44: 2413-2417.

Borges W, Mancilla G, Guimaraes D, Patron R, Collado I, Pup M (2011) Azaphilones from the endophyte Chaetomium globosum. Journal of Natural Products, 74: 1182-1187.

Botic T, Kuncic M, Sepcic K, Knez Z, Gunde-Cimerman (2012) Salt induces biosynthesis of hemolytically active compounds in the xerotolerant food-borne fungus Wallemia sebi. FEMS Microbiology Letters, 36: 40-46.

Boyd R, McAlees A, Taylor A, Walter J (1991) Isolation of new isocyanide metabolites of Trichoderma hamatum as their ( $\eta 5$-pentamethylcyclopentadienyl)- or (5-ethyltetra methylcyclopentadienyl)bis( $\mu$-thiocyanato)rhodium complexes. Journal of the Chemical Society, Perkin Transactions 1, 6: 1461-1465.

Brewer D, Duncan W, Jerram C, Leach K, Safe S, Taylor A, Vining LC, Archibald R, Stevenson RG, Mirocha CJ, Christensen CM (1972) Ovine ill-thrift in Nova Scotia. 5. The production and toxicology of chetomin, a metabolite of Chaetomium spp. Canadian Journal of Microbiology, 18: 1129-1137.

Brewer D, Feicht A, Taylor A, Keeping J, Taha A, Thaller V (1982) Ovine ill-thrift in Nova Scotia. 9. Production of experimental quantities of isocyanide metabolites of Trichoderma hamatum. Canadian journal of microbiology, 28:1252-1260.

Brewer D, Gabe E, Hanson A, Taylor A (1979) Isonitrile acids from cultures of the fungus Trichoderma hamatum (Bon.) Bain. aggr., X-ray structure. Chemical Communications, 1979: 1061-1062.

Brewer D, Jerram WA, Meiler D, Taylor A (1970) The toxicity of cochliodinol, an antibiotic metabolite of Chaetomium spp.. Canadian Journal of Microbiology, 16: 433-440. 
Brewer D, Jerram W, Taylor A (1968) The production of cochliodinol and a related metabolite by Chaetomium species. Canadian Journal of Microbiology, 14: 861-866.

Brewer D, Mason FG, Taylor A (1987) The production of alamethicins by Trichoderma spp. Canadian Journal of Chemistry, 33: 619-625.

Brewer D and Taylor A (1978) The production of toxic metabolites by Chaetomium spp. isolated from soils of permanent pasture. Canadian Journal of Microbiology, 24: 1082-1086.

Brian PW and Hemming HG (1945) Gliotoxin, a fungistatic metabolic product of Trichoderma viride. Annals of Applied Biology, 32: 214-220.

Bunkers G, Kenfield D, Strobel G, Sugawara F (1990) Structure-activity relationships of the eremophilanes produced by Drechslera gigantea. Phytochemistry, 29: 1471-1474.

Bush R and Portnoy JM (2001) The role and abatement of fungal allergens in allergic diseases. Journal of Allergy and Clinical Immunology, 107: 430-442.

Bushley K, Raja R, Jaiswal P, Cumbie J, Nonogaki M, Boyd A, ...Spatafora J (2013). The Genome of Tolypocladium inflatum: Evolution, Organization, and Expression of the Cyclosporin Biosynthetic Gene Cluster. PLoS Genetics, 9: e1003496.

Capasso R, Iacobellis N, Bottalico A, Randazzo G (1984) Structure-toxicity relationships of the eremophilane phomenone and PR-toxin. Phytochemistry, 23:2781-2784.

Capasso R, Palumbo G, Randazzo G (1986) Stereostructure and formation mechanism of a new substituted benzofuran from phomenone. Tetrahedron, 42: 4493-4498.

Cardoza R, Malmierca M, Hermosa M, Alexander N, McCormick S, Proctor R, Tijerino A, Rumbero A, Monte E, Gutierrez S (2011) Identification of loci and functional characterization of trichothecene biosynthesis genes in filamentous fungi of the genus Trichoderma Applied and Environmental Microbiology, 77: 4867-4877.

Chaverri P and Samuels GJ (2003) Hypocrea/Trichoderma (Ascomycota, Hypocreales, Hypocreaceae): species with green ascospores. Studies in Mycology, 48: 1-36.

Christensen M, Frisvad JC, Tuthill D (1999) Penicillium miczynskii and related species. Mycological Research, 103: 527-541.

Clay K (1988) Fungal endophytes of grasses: a defensive mutualism between plants and fungi. Ecology, 69: 10-16.

Cole R and Schweikert M (2003) Handbook of secondary metabolites volume I. Elseiver Science, London . 
Colins R and Halim A (1972) Characterization of the major aroma constituent of the fungus Trichoderma viride. Journal of Agriculture and Food Chemistry, 20: 437-438.

Corley D, Miller-Wideman M, Durley R (1994) Isolation and structure of harzianum A: A new trichothecene from Trichoderma harzianum. Journal of Natural Products, 57: 442-425.

Cox RH, Hernandex O, Dorner JW, Cole RJ, Fennell DI (1979) A new isochroman mycotoxin isolated from Penicillium steckii. Journal of Agricultural and Food Chemistry, 5: 999-1001.

Crameri R (2011) The problem of cross-reactivity in the diagnosis of fungal allergy. Clinical Experimental Allergy, 41: 302-304.

Cragg GM and Newman DJ (2005) Biodiversity: a continuing source of novel drug leads. Pure Applied Chemistry, 77: 7-24.

Cutler H, Arrendale R, Cole P, Davis E, Cox R (1989) 3,7-Dimethyl-8-hydroxy-6-methoxyisochroman from Penicillium corylophilum: plant growth regulatory activity. Agricultural and Biological Chemistry, 53: 1975-1977.

Cutler H, Cutler S, Ross S, Sayed K, Dugan F, Bartlett M, Hill A, Hill R, Parker S. 1999. Koninginin G, a new metabolite from Trichoderma aureoviride. Journal of Natural Products, 62: 137-139.

Cutler H, Himmelsbach D, Arrendale R, Cole P, Cox R (1989) Koninginin A: a novel plant growth regulator from Trichoderma koningii. Agricultural and Biological Chemistry, 53: 26052611.

Cutler H, Majetich G, Tian X, Spearing P (1997) Syntheses of 3,7-dimethyl-8-hydroxy-6methoxyisochroman, the 3,7-dimethyl-6-hydroxy-8-methoxy isomer, and their ester and ether derivatives: plant growth regulatory activity. Journal of Agricultural and Food Chemistry, 45: 1422-1429.

Cutler H, Himmelsbach D, Yagen B, Arrendale R, Jacyno J, Cole P, Cox R (1991) Koninginin B: A biologically active congener of koninginin A from Trichoderma koningii. Journal of Agricultural Food Chemistry, 39: 977-980.

Dales R, Burnett R, Zwanenburg H (1991) Adverse health effects among adults exposed to home dampness and molds. The American Review of Respiratory Disease, 143: 505-509.

Dannemiller K, Mendell M, Macher J, Kumagai K, Bradman A, Holland N, Harley K, Eskenazi B, Peccia J (2014) Next-generation DNA sequencing reveals that low fungal diversity. Indoor Air, DOI: 10.1111.

Davies J (1990). What are antibiotics? Archaic functions for modern activities. Molecular microbiology, 4: 1227-1232. 
Degenkolb T, Dieckmann R, Nielsen KF, GGrafenhan T, Theis C, Zafari D, Chaverri P, Ismaiel A, Bruckner H, Dohren H, Thrane U, Petrini O, Samuels G (2008) The Trichoderma brevicompactum clade: a separate lineage with new species, new peptaibiotics and mycotoxins. Mycological Progress, 7: 177-219.

Degenkolb T, Kirschbaum J, Bruckner H (2007) New sequences, constituents, and producers of peptaibiotics: an updated review. Chemistry and biodiversity, 4: 1052-1067.

De La Campa R, Seifert K, Miller JD (2007) Toxins from strains of Penicillium chrysogenum isolated from buildings and other sources. Mycopathologia, 163:161-168.

Demain A and Fang A (2000) The natural functions of secondary metabolites. History of Modern Biotechnology I. Springer Berlin Heidelberg, 1-39.

Desroches T, McMullin D, Miller JD (2014) Extrolites of Wallemia sebi, a very common fungus in the built environment. Indoor Air: 10.1111

Ding G, Song Y, Chen J, Xu C, Ge H, Wang X, Tan R (2006) Chaetoglobosin U, a cytochalasan alkaloid from endophytic Chaetomium globosum IFB-E019. Journal of Natural Products, 69: 302-304.

Domsch KH, Gams W, Anderson TH (2007) Compendium of soil fungi, 2nd ed. IHW, Eching.

Dos Santos C, da Costa G, Figueroa-Villar G (2012) Identification of citrinin as the defense metabolite of Penicillium corylophilum stressed with the antagonist fungus Beauveria bassiana. Natural Product Research, 1: 1-7.

Drew S and Demain A (1977) Effect of primary metabolites on secondary metabolism. Annual Reviews in Microbiology, 31: 343-356.

Druzhinina I Kopchinskiy A, Kubicek C (2006) The first 100 Trichoderma species characterized by molecular data. Mycoscience, 47: 55-64.

Dunlop R, Simon A, Sivasithamparam K, Ghisalberti E (1989) An antibiotic from Trichoderma koningii active against soilborne plant pathogens. Journal of Natural Products, 52: 67-74.

El-Kady I, Abdel-Mallek A, El-Maraghy S, Hassan H (1994? Toxigenic moulds in pesticidetreated liquid medium. Cryptic Mycology, 15:76-81.

Engelhart S, Loock A, Skutlarek D, Sagunski H, Lommel A, Farber H, Exner M (2002) Occurrence of toxigenic Aspergillus versicolor isolates and sterigmatocystin in carpet dust from damp indoor environments. Applied Environmental Microbiology, 68: 3886-3890. 
Evidente A, Andolfi A, Cimmino A, Ganassi S, Altomare C, Favilla M, Christofaro A, Vitagliano S, Sabatini M (2009) Bisorbicillinoids produced by the fungus Trichoderma citrinoviride affects feeding preference of the aphid Schizaphis graminum. Journal of Chemical Ecology, 35: 533-341.

Evidente A, Ricciardiello G, Andolfi A, Sabatini M, Ganassi S, Altomare C, Favilla M, Melck D (2008) Citrantifidiene and citrantifidol: bioactive metabolites produced by Trichoderma citrinoviride with potential antifeedant activity towards aphids. Jounral of Agriculture and Food Chemistry, 56: 3569-3573.

Faquim-Mauro E, Jacysyn J, Macedo M (2003) Anaphylactic and non-anaphylactic murine IgG1 differ in their ability to bind to mast cells. Immunobiology, 207: 169-177.

Flannigan B, McCabe E, McGarry F (1991) Allergenic and toxigenic micro-organisms in houses. Journal of Applied Bacteriology, 70: 61-73.

Flannigan B, Samson RA, Miller JD (2001) Microorganisms in home and indoor work environments: diversity, health impacts, investigation and control. London, UK: Taylor \& Francis.

Flannigan B and Miller JD (2011) Microbial growth in indoor environments. In: Flannigan B, Samson RA, Miller JD (eds) Microorganisms in home and indoor work environments: diversity, health impacts, investigation and control, 2nd edn. Taylor \& Francis, New York, pp 57-107.

Flemming J, Hudson B, Rand TG (2003) Comparisons of inflammatory and cytotoxic lung responses in mice after intratracheal exposure to spores of two different Stachybotrys chartarum strains. Toxicological Sciences, 78: 267-275.

Fogle M, Douglas D, Jumper C, Straus DC (2007) Growth and mycotoxin production by Chaetomium globosum. Mycopathologia, 164: 49-56.

Fogle M, Douglas D, Jumper C, Straus D (2008) Growth and mycotoxin production by Chaetomium globosum is favored in a neutral $\mathrm{pH}$. International Journal of Molecular Science, 9: 2357-2365.

Fonzi W and Sypherd P (1986) Trichodermin esterase activity and trochodermin resistance in Mucor racemosus. Antimicrobial Agents and Chemotherapy, 29: 570-575.

Foto M, Vrijmoed L, Miller JD, Ruest K, Lawton M, Dales RE (2005) A comparison of airborne ergosterol, glucan and Air-O-Cell data in relation to physical assessments of mold damage and some other parameters. Indoor Air, 15: 257-266.

Fox E and Howlett B (2008). Secondary metabolism: regulation and role in fungal biology. Current opinions in microbiology, 11: 481-487. 
Frank M and Hess E (1941) Studies on salt fish: V. Studies on Sporendonema epizoum from"Dun" salt fish. Journal of the Fisheries Board of Canada, 5: 276-286.

Frank M, Kingston E, Jeffery J, Moss M, Murray M, Simpson T, Sutherland A (1999) Walleminol and walleminone, novel caryophyllenes from the toxigenic fungus Wallemia sebi. Tetrahedron Letters, 40: 133-136.

Frisvad JC, Andersen B, Thrane U (2008) The use of secondary metabolite profiling in chemotaxonomy of filamentous fungi. British Mycological Society, 112: 231-240.

Frisvad JC and Filtenborg O (1990) Secondary metabolites as consistent criteria in Penicillium taxonomy and a synoptic key to Penicillium subgenus Penicillium. In: Sampson RA, Pitt JI, eds. Modern concepts in Penicillium and Aspergillus classification. New York: Plenum Press. p 373384.

Frisvad JC and Thrane U (1995) Mycotoxin production by food-borne fungi. In: Introduction to Food-Borne Fungi. R.A. Samson, E.S. Hoekstra, J.C. Frisvad, O. Filtenborg (Eds.), Centraalbureau voor Schimmelcultures, Delft pp. 251-261

Fujiwara A, Okudu T, Masuda S, Shiomi Y, Miyamoto C, Sekine Y, Tazoe M, Fujiwara M (1982) Fermentation, isolation and characterization of isonitrile antibiotics. Agricultural and Biological Chemistry, 46: 1803-1809.

Germain A, Bruggemeyer D, Zhu J, Genet C, O’Brein P, Porco J (2011) Synthesis of the azaphilones $(+)$-sclertiorin and the $(+)-8$-)-methylsclerotiorinamine utilizing $(+)$-sparteine surrogates in Copper-mediated oxidative dearomatization. Journal of Organic Chemistry, 76: 2577-2584.

Ghisalberti E and Rowland C (1993) Antifungal metabolites from Trichoderma harzianum. Journal of Antibiotics, 56: 1799-1804.

Gloer J (2007) Applications of fungal ecology in the search for new bioactive natural products. Environmental and Microbial Relationships, 2nd Edition, 257-283.

Godish T and Godish D (2006). Mold infestation of wet spray-applied cellulose insulation. Journal of the Air and Waste Management Association, 56: 90-95.

Godtfredsen W and Vangedal S (1964) Trichodermin, a new antibiotic, related to trichothecin. Journal of the Chemical Society, Perkin Transactions, London, 188.

Golder WS andWatson TR (1980) Lanosterol derivatives as precursors in the biosynthesis of viridin. Part 1. Journal of the Chemical Society, Perkin Transactions 1, 2: 422-425.

Gordon W, Johanning E, Haddad L (1999) Cognitive impairment associated with exposure to toxigenic fungi. In: Johanning (Ed) Bioaerosols, Fungi and mycotoxins: health effects, 
assessment, prevention and control. Eastern New York Occupational and Environmental Health Center. New York pp 94-98.

Grabley S, Granzer E, Hutter K, Ludwig D, Mayer M, Thiericke, Till G, Wink J (1992)

Secondary metabolites by chemical screening. 8. Decarestrictines, a new family of inhibitors of cholesterol biosynthesis from Penicillium. I. Strain description, fermentation, isolation and properties. Journal of Antibiotics, 45: 56-65.

Green B, Tovey E, Sercombe J, Blachere F, Beezhold D, Schmechel D (2006) Airborne fungal fragments and allergenicity. Medical Mycology, 1:245-255.

Gunde-Cimerman N, Ramos J, Plemenitas A (2009) Halotolerant and halophilic fungi. British Mycological Society, 113: 1231-1241.

Hanson JR (2007) Diterpenoids. Natural Product Reports, 24: 1332-1341. Hanson A, McAiees A,Taylor A (1985) Ovine ill-thrift in Nova Scotia. Part 10. Palladium and rhodium complexes as reagents in the investigation of isocyanide metabolites of Trichoderma hamatum. Journal of the Chemical Society, Perkin Transactions 1, 441-446.

Harman G, Howell C, Viterbo A, Lorito M (2004) Trichoderma species- opportunistic, avirulent symbionts. Nature Reviews in Microbiology, 2: 43-56.

Hawksworth DL (2001) The magnitude of fungal diversity: the 1.5 million species estimate revisited. Mycological Research, 105: 1422-1432.

Hawksworth DL (1991) The fungal dimension of biodiversity: magnitude, significance, and conservation. Mycological Research, 95: 641-655.

Hawksworth DL, Crous P, Redhead S, Reynolds D, Samson R, Seifert K, ...Kõljalg U (2011). The Amsterdam declaration on fungal nomenclature. IMA Fungus: The Global Mycological Journal, 2: 105-112.

Health Canada (2004) Fungal contamination in public buildings: Health effects and investigation methods. Ottawa: Health Canada.

Health Canada (2007) Residential indoor air quality guidelines: moulds. Health Canada, Ottawa, Ontario.

Hodgson M, Morey P, Leung W, Morrow L, Miller JD, Jarvis B, Robbins H, Halsey J, Storey E (1998) Building associated pulmonary disease from exposure to Stachybotrys chartarum and Aspergillus versicolor. Journal of Occupational and Environmental Medicine, 40: 241-249.

Houbraken J, Frisvad JC, Samson RA (2011) Fleming's penicillin producing strain is not Penicillium chrysogenum but P. rubens. International Mycological Association, 2: 87-95. 
Houbraken J and Sampson RA (2011) Phylogeny of Penicillium and the segregation of Trichocomaceae into three families. Studies in Mycology, 70: 1-51.

Inagaki T, Kaneda K, Suzuki Y, Hirai, H, Nomura E, Sakakibara T, Yamauchi Y, Huang L, Norcia M, Wondrack L, Sutcliffe J, Kojima N (1998) CJ-12,373, a novel topoisomerase II inhibitor: fermentation, isolation, structure elucidation and biological activities. Journal of Antibiotics, 51: 112-116.

Isaka M, Jaturapat A, Kladwang W, Punya J, Lertwerawat Y, Tanticharoen M, Thebtaranonth Y (2000) Antiplasmodial compounds from the wood decayed fungus Xylaria sp. BCC 1067. Planta Medica, 66: 473-475.

Ito M, Tsukida K, Toube T (1981) Pyrrolylpolyenes. Part3. Synthesis of all-(E)-wallemia C and stereochemistry of natural wallemia C. Journal of the Chemical Society, Perkin Transactions 1, 1981: 3255-3257.

Jaklitsch W, Samuels G, Dodd S, Lu B, Druzhinina I (2006) Hypocrea rufa/Trichoderma viride: a reassessment, and description of five closely related species with and without warted conidia. Studies in Mycology, 56: 135-177.

Jarvis B and Miller JD (2005) Mycotoxins as harmful indoor air contaminants. Applied Microbiology and Biotechnology, 66: 367-372.

Jarvis B, Sorenson W, Hintikka E-L, Nikulin M, Zhou Y, Jiang J, Wang S, Hinkley S, Etzel R, Dearborn D (1998) Study of toxin production by isolates of Stachybotrys chartarum and Memnoniella echinata isolated during a study of pulmonary hemosiderosis in infants. Applied and Environmental Microbiology, 64:3620-3625.

Jarvis B, Zhou Y, Jiang J, Wang S, Sorenson W, Hintikka E-L, Nikulin M, Parikka P, Etzel R, Dearborn D (1996) Toxigenic molds in water-damaged buildings: dechlorogriseofulvins from Memnoniella echinata. Journal of Natural Products Rapid Communications, 6: 553-554.

Jiao W, Feng Y, Blunt J, Cole A, Munro M (2004) Chaetoglobosins Q, R, and T, three further new metabolites from Chaetomium globosum. Journal of Natural Products, 10: 1722-1725.

Johnson L and Miller JD (2012) Consequences of large scale production of marijuana in residential buildings. Indoor Built Environment, 21: 595-600.

Keller NP, Turner G, Bennett J. (2005) Fungal secondary metabolism - from biochemistry to genomics. Nature Reviews in Microbiology, 3: 937-947.

Kendrick B (2000) The fifth kingdom, $3^{\text {rd }}$ edition. Newburyport, Massachusetts.

Kendrick B (2001) Fifth Kingdom, Third Edition. Mycologue Publications, Sidney, BC. 
Kildeso J, Wurtz H, Nielsen K, Kruse P, Wilkins K, Thrane U, Gravensen P, Nielsen P, Schneider T (2003) Determination of fungal spore release from wet building materials. Indoor Air, 13: 148-155.

Kimura Y, McGahren W, Suzuki A, Tamura S (1978) Structure of a new fungal pyrone, from an unidentified Penicillium sp. Journal of Agricultural and Biological Chemistry, 42: 1625-1626.

Kobayashi Y, Tan C, Kishi Y (2000) Toward the creating of a universal database for the stereochemical assignment: the case of 1, 3, 5-trisubstuted acyclic systems. Helvetica Chemica Acta, 83: 2562-2571.

Kogej T, Gostincar C, Volkmann M, Gorbushina A, Gunde-Cimerman N (2006) Mycosporines in extremophilic fungi- novel complementary osmolytes? Environmental Chemistry, 3: 105-110.

Kontani M, Sakagami Y, Marumo S (1994) First beta-1, 6- glucan biosynthesis inhibitor, bisvertinolone isolated from fungus, Acremonium strictum and its absolute stereochemistry. Tetrahedron Letters 35:2577-2580.

Kosalec I, Safranic A, Pepeljnjak S, Druzina V, Ramic S, Kopjar N (2008) Genotoxicity of tryptophol in a battery of short term assays on human white blood cells in vitro. Basic and clinical pharmacology and toxicology, 102: 443-452.

Kosemura S (2003) Meroterpenoids from Penicillium citreo-viride B. IFO 4692 and 6200 hybrid. Tetrahedron, 59: 5055-5072.

Kosemura S, Miyata H, Matsunaga K, Yamamura S (1992) Biosynthetic studies of citreohybridones, the metabolites of a hybrid strain KO 0031 derived from Penicillium citreoviride B. IF0 6200 and 4692. Tetrahedron Letters, 33: 3883-3886.

Kosemura S, Miyata H, Yarnamura S, Albone K, Simpson T (1994) Biosynthetic studies on citreohybridones, metabolites of a hybrid strain KO 0031 derived from Penicillium citreo-viride B. IF0 6200 and 4692. Journal of the Chemical Society, Perkin Transactions 1, 1994: 135-139.

Koyama N, Ohshiro T, Tomoda H, Omura S (2007) Fungal isobisvertinol, a new inhibitor of lipid droplet accumulation in mouse marcophages. Organic Letters, 9: 425-428.

Kozlovskii AG, Zhelifonova VP, Antipova TV (2013) Fungi of the genus Penicillium as producers of physiologically active compounds. Applied Biochemical Microbiology, 1: 1-10.

Kredics L, Hatvani L, Manczinger L, Vagvolgyi C, Antal Z (2011) Trichoderma. In The Molecular Detection of Human Fungal Pathogens (Liu D, ed.) CRC Press, Taylor \& Francis Group, London pp. 509-526. 
Kremer AM, Pal TM, de Monchy JG, Kauffman HF, de Vries K (1989) Precipitating antibodies and positive skin tests in workers exposed to airborne antigens from a contaminated humidification system. International Archives of Occupational Health, 61:547-553.

Kuncic M, Kogej T, Drobne D, Gunde-Cimerman N (2010). Morphological response of the halophilic fungal genus Wallemia to high salinity. Applied Environmental Microbiology, 76: 329-337.

Lai S, Shizuri Y, Yamamura S, Kawai K, Furukawa H (1991) Three new phenolic metabolites from Penicillium species. Heterocycles, 32: 297-305.

Lappalainen S, Pasanen A, Reiman M, Kalliokoski P (1998) Serum IgG antibodies against Wallemia sebi and Fusarium species in Finnish farmers. Annals of Allergy, Asthma an Immunology, 82: 585-592.

Larsen T, Smedsgaard J, Nielsen KF, Hansen M, Frisvad JC (2005) Phenotypic taxonomy and metabolite profiling in microbial drug discovery. Natural Product Reports, 22: 672-695.

Leech J, Nelson W, Burnett R, Aaron S, Raizenne M (2002) It's about time: A comparison of Canadian and American time activity patterns. Journal of Exposure and Analytical Environmental Epidemiology, 12: 427-432.

Leitgeb B, Szekers A, Manczinger L, Vagvolgyi C, Kredics L (2007) The history of alamethicin: a review of the most extensively studied petaibol. Chemistry and biodiversity, 4: 1027-1051.

Lian X, Lackner M, deHoog G, Gerrits van de Ende A, Priha O, Suihko M, Houbraken J, Varga J, Samson R, Malarstig B, Thompson P, Stott R, Richardson M (2011) Assessment of identity of filamentous fungi colonizing water-damaged building materials. Sydowia, 63: 49-66.

Liang Y, Zhao W, Xu J, Miller JD (2011) Characterization of two related exoantigens from the biodeteriogenic fungus Aspergillus versicolor. International Biodeterioration and Biodegradation, 65: 217-226.

Liu G and Wang Z (2001) Total synthesis of koninginin D, B and E. Synthesis, 2001: 119-127.

Lopes F, Steindorff A, Geraldine A, Brandao R, Monteiro V, Junior M, Coelho A, Ulhoa C, Silva R (2012) Biochemical and metabolic profiles of Trichoderma strains isolated from common bean crops in the Brazilian Cerrado, and potential antagonism against Sclerotinia sclerotiorum. Fungal Biology, 116: 815-824.

Lubeck M, Poulsen SK, Lubeck PS, Jensen DF, Thrane U (2000) Identification of Trichoderma strains from building materials by ITS1 ribotyping, UP-PCR fingerprinting and UP-PCR cross hybridization. FEMS Microbioliobogy Letters 185: 129-134. 
Luo W, Wilson AM, Miller JD (2010) Characterization of a $52 \mathrm{kDa}$ exoantigen of Penicillium chrysogenum and monoclonal antibodies suitable for its detection. Mycopathologia, 169: 15-26.

Lustgraaf B (1978) Seasonal abundance of xerophilic fungi and house-dust mites (Acarida: Pyroglyphidae) in mattress dust. Oecologia, 36: 81-91.

Maddau L, Cabras A, Franceschini A, Linaldeddu B, Crobu S, Roggio T, Pagnozzi D (2006) Occurrence and characterization of peptaibols from Trichoderma citrinoviride, an endophytic fungus of cork oak, using electrospray ionization quadrupole time-of-flight mass spectrometry. Microbiology, 155: 3371-3381.

Malmström J, Christopherson C, Frisvad J (2000) Secondary metabolites characteristic of Penicillium citrinum, Penicillium steckii and related species. Phytochemistry, 54: 301-309.

Mann J (1987) Secondary Metabolism Second Addition. Oxford Science Publications. Clarendon Press Oxford. 1987.

Maskey R, Grun-Wollny I, Laatsch H (2005) Sorbicillin analogs and related dimeric compounds from Penicillium notatum (2005) Journal of Natural Products, 68: 865-870.

Matheny P, Gossmann J, Zalar P, Kumar T, Hibbett D (2006) Resolving the phylogenetic position of the Wallemiomycetes: an enigmatic major lineage of Basiodiomycota. Canadian Journal of Botany, 84: 1794-1805.

Matsuda Y, Awakawa A, Abe I (2013) Reconstituted biosynthesis of fungal meroterpenoid andrastin A. Tetrahedron, 69: 8199-8204.

McGregor H, Miller JD, Rand T, Solomon J (2008) Mold ecology: recovery of fungi from certain moldy building materials. In B. Prezant, D. Weekes, \& J. Miller, Recognition, evaluation, and control of indoor mold. Fairfax: American Industrial Hygiene Association.

Mendell MJ, Mirer AG, Cheung K, Tong M, Douwes J (2011) Respiratory and allergic health effects of dampness, mold, and dampness-related agents: a review of the epidemiologic evidence, Environmental Health Perspectives, 119: 748-556.

McMullin DR, Nsiama TK, Miller JD (2014) Isochromans and $\alpha$-pyrones from Penicillium corylophilum. Journal of Natural Products, Accepted.

McMullin DR, Tienabe K. Nsiama and J. David Miller (2014) Secondary metabolites from Penicillium corylophilum isolated from damp buildings. Mycologia. Accepted.

McMullin DR, Sumarah MW, Miller JD (2012) Chaetoglobosins and azaphilones produced by Canadian strains of Chaetomium globosum isolated from the indoor environment. Mycotoxin Research, 29: 47-54. 
McMullin DR, Sumarah MW, Blackwell BA, Miller JD (2013) New azaphilones from Chaetomium globosum isolated from the built environment. Tetrahedron Letters, 54: 568-572.

McRae C, Hocking A, Seppelt RD (1999) Penicillium species from terrestrial habitats in the Windmill Islands, East Antarctica, including a new species, Penicillium antarcticum. Polar Biology, 21: 97-111.

Michael A, Grace E, Kotiw M, Barrow R (2003) Isochromophilone IX, a novel GABAcontaining metabolite isolated from a cultures fungus, Penicillium sp. Australian Journal of Chemistry, 56: 13-15.

Mikkola R, Andersson M, Kredics L, Grigoriev P, Sundell N, Salkinoja-Salonen M (2012) 20Residue and 11-residue peptaibols from the fungus Trichoderma longibrachiatum are synergistic in forming $\mathrm{Na}+/ \mathrm{K}+-$ permeable channels and adverse action towards mammalian cells. FEBS, 279: 4172-4190.

Miller JD (1992) Fungi as contaminants in indoor air. Atmospheric Environment. Vol. 26A. No. 12. 2163-2172.

Miller JD (2011) Foliar endophytes of spruce species found in the Acadian forest: basis and potential for improving the tolerance of the forest to spruce budworm. Endophytes of forest trees: biology and applications. Springer, New York. pp. 237-249.

Miller JD and Day JD (1997) Indoor mold exposure: epidemiology, consequences and immunotherapy, Canadian Journal of Allergy and Clinical Immunology, 2: 25-32.

Miller JD, Duandzic R, Frescura A (2007) Indoor- and outdoor-derived contaminants in urban and rural homes in Ottawa, Ontario, Canada. Journal of Air and Waste Management Association, 57: 297-302.

Miller JD, Greenhalgh R, Wang Y, Lu M (1991) Mycotoxin chemotypes of three Fusarium species. Mycologia, 83: 121-130.

Miller JD, Rand T, Jarvis, B (2003) Stachybotrys chartarum: cause of human disease or media darling? Medical Mycology, 41: 271-291.

Miller JD, Rand T, McGregor H, Solomon J, Yang J (2008) Mold ecology: recovery of fungi from certain moldy building materials. Prezant B, Weekes D, Miller JD (eds) Recognition, Evaluation and Control of Indoor Mold. American Industrial Hygiene Association, Fairfax, VA.

Miller JD, Sun M, Gilyan A, Roy J, Rand TG (2010) Inflammation-associated gene transcription and expression in mouse lungs induced by low molecular weight compounds from fungi from the built environment. Chemico-Biological Interactions, 183: 113-124. 
Ming H, Zhang W, Ding G, Saparpakorn P, Song Y, Hannongbua S, Tan R (2008)

Chaetoglobins A and B, two unusual alkaloids from endophytic Chaetomium globosum culture.

Chemical Communications, 45: 5978-5980.

Mori K and Abe K (1995) Synthetic microbial chemistry XXVIII. Synthesis and absolute configuration of (-)-koninginin A. European Journal of Organic Chemistry, 6: 943-948.

Moss M, Jackson R, Rogers D (1975) The characterization of 6-(pentyl-1-enyl)-alpha pyrone from Trichoderma viride. Phytochemistry Reviews. 14: 2706-2708.

Moule Y, Moreau S, Bousquet J (1977) Relationships between the chemical structure and the biological properties of some eremophilane compounds related to PR toxin. Chemical-Biological Interactions, 17: 185-192.

Muroga Y, Yamada T, Numata A, Tanaka R (2009) Chaetomugilins I-O, new potent cytotoxic metabolites from a marine-fish derived Chaetomium species. Stereochemistry and biological activities. Tetrahedron, 65:7580-7586.

Nakazawa T, Ishiuchi K, Sato M, Tsunematsu Y, Sugimoto S, Gotanda Y, Noguchi H, Hotta K, Watanabe K (2013) Targeted Disruption of Transcriptional Regulators in Chaetomium globosum Activates Biosynthetic Pathways and Reveals Transcriptional Regulator-Like Behavior of Aureonitol. Journal of the Americain Chemical Society, 136: 13446-13455.

National Academy of Science (2000) Clearing the air: Asthma and indoor air exposures, NAS Institute of Medicine, National Academy Press, Washington DC.

Neveu WA, Bernardo E, Allard JL, Nagaleekar V, Wargo MJ, Davis RJ, Iwakura Y, Whittaker LA, Rincon M (2011) Fungal allergen beta-glucans trigger p38 mitogen-activated protein kinasemediated IL-6 translation in lung epithelial cells. American Respiratory Cell and Molecular Biology, 45: 1133-1141.

Nicolaou K, Simonsen K, Vassilikogiannakis G, Baran P, Vidali V, Pitsinos E, Couladouros EA (1999) Biomimetic explorations towards the bisorbicillinoids: total synthesis of isorbicillinol, bisorbibutenolide, and trichodimerol. Angew Chem Int Ed 38:3555-3559

Nielsen KF (2003) Mycotoxins production by indoor molds. Fungal Genetics and Biology, 39: 103-117.

Nielsen KF, Dalsgaard PW, Smedsgaard J, Larsen TO (2005) Andrastins A-D, Penicillium roqueforti metabolites consistently produced in blue-mold-ripened cheese. Journal of Agricultural and Food Chemistry, 53: 2908-2913.

Nielsen KF, Grafenhan T, Zafari D, Thrane U (2005) Trichothecene production by Trichoderma brevicompactum. Agriculture and Food Chemistry, 53: 8190-8196. 
Nielsen KF, Gravesen S, Nielsen P, Andersen B, Thrane U, Frisvad JC (1999) Production of mycotoxins on artificially and naturally infested building materials. Mycopathologia, 145: 43-56.

Nielsen KF, Hansen M, Larsen T, Thrane U (1998) Production of trichothecene mycotoxins on water damaged gypsum boards in Danish buildings. International Biodeterioration and Biodegradation, 42:1-7.

Nielsen KF, Holm G, Uttrup L, Nielsen P (2004) Mould growth on building materials under low water activities. Influence of humidity and temperature on fungal growth and secondary metabolism. International Biodeterioration and Biodegradation, 54: 325-336.

Nielsen KF, Smedsgaard J, Larsen T, Lund F (2003) Chemical identification of fungi: metabolite profiling and metabolomics. Marcel Dekker, Inc. New York, N.Y.

Nielsen KF, Sumarah MW, Frisvad JC, Miller JD (2006) Production of metabolites by species in the Penicillium roqueforti complex. Journal of Agricultural and Food Chemistry, 54: 3756-3763.

Nielsen KF and Thrane U (2001) A fast method for detection of trichothecenes in fungal cultures using gas chromatography tandem mass spectrometry. Journal of Chromatography, 929: 75-87.

Nielsen KF, Thrane U, Larsen TO, Nielsen PA, Gravesen S (1998) Production of mycotoxins on artificially inoculated building materials. International Biodeterioration and Biodegradation, 42: $1-7$.

NIOSH (2012) National Institute for Occupational Safety and Health: preventing occupational respiratory disease from exposures caused by dampness in office buildings, schools, and other nonindustrial buildings. NIOSH Publication \# 2013-102. Cincinnati, OH: NIOSH.

Nonnenmann M, Coronado G, Thompson B, Griffith W, Hanson J, Vesper S, Faustman E (2012) Utilizing pyrosequencing and quantitative PCR to characterize fungal populations among house dust samples. Jounral of Environmental Monitoring, 14: 2038-2043.

O'Brien M, Nielsen K, O'Kiely P, Forristal P, Fuller H, Frisvad J (2006) Mycotoxins and other secondary metabolites produced in vitro by Penicillium paneum Frisvad and Penicillium roqueforti Thom isolated from baled grass silage in Ireland. Journal of Agriculture and Food Chemistry, 54: 9268-9276.

Ohnishi T, Yamada G, Tanaka H, Nakajima K, Tanaka S, Morita-Ichimura S, Takahashi R, Sato M, Shibusa T, Abe S (2002) A case of chronic hypersensitivity pneumonia with elevation of serum SP-D and KL-6. Journal of the Japanese Respiratory Society, 40: 66-70.

Ohtsubo K, Saito M, Sekita S, Yoshihira K, Natori S (1978) Acute toxic effects of chaetoglobosin A, a new cytochalasan compound produced by Chaetomium globosum, on mice and rats. Japanese Journal of Experimental Medicine, 48: 105-110. 
Osmanova N, Schultze W, Ayoub N (2010) Azaphilones: a class of fungal metabolites with diverse biological activity. Phytochemistry Reviews, 9: 315-342.

Overy DP, Larsen TO, Dalsgaard PW, Frydenvang K, Phipps R, Munro MH, Christophersen C (2005) Andrastin A and barceloneic acid metabolites, protein farnesyl transferase inhibitors from Penicillium albocoremium chemotaxonomic significance and pathological implications. Mycological Research, 109: 1243-1249.

Padamsee M, Kumar T, Riley R, Binder M, Boyd A, Calvo A,... Aime M (2012) The genome of the xerotolerant mold Wallemia sebi reveals adaptations to osmotic stress and suggests cryptic sexual reproduction. Fungal Genetics and Biology, 49: 217-226.

Park JH, Choi GJ, Jang KS, Lim HK, Kim HT, Cho KY, Kim JC (2005) Antifungal activity against plant pathogenic fungi of chaetoviridins isolated from Chaetomium globosum. FEMS Microbiology Letters, 252: 309-313.

Parker SR, Cutler HG, Schreiner PR (1995) Koninginin E: isolation of a biologically active natural product from Trichoderma koningii. Bioscience, Biotechnology and Biochemistry, 59: 1747-1749.

Peng X, Wang L, Liu P, Hong K, Chen H, Yin X, Zhu W (2011) Aromatic compounds from the halotolerant fungal strain of Wallemia sebi PXP-89 in a hypersaline medium. Archives in Pharmaceutical Research, 34: 907-912.

Pieckova E (2003) In vitro toxicity of indoor Chaetomium Kunze ex Fr. Annals of Agricultural and Environmental Medicine, 10: 9-14.

Pitt J (1979) The genus Penicillium and its teleomorphic states Eupenicillium and Talaromyces. London, UK: Academic Press. pp. 634.

Pitt J and Hocking A (2009) Fungi and food spoilage. New York: Springer.

Pitt J and Hocking A (1977) Influence of solute and hydrogen ion concentration on the water relations of some xerophilic fungi. Journal of General Microbiology, 101: 35-40.

Polizzi V, Delmulle B, Adams A, Moretti A, Susca A, Picco A, Rosseel Y, Kindt R, Bocxlaer J, De Kimpe N, Van Peteghem C (2009) JEM Spotlight: Fungi, mycotoxins and microbial volatile organic compounds in mouldy interiors from water-damaged buildings. Journal of Environmental Monitoring, 11: 1849-1858.

Provost N, Shi C, She Y, Cyr T, Miller JD (2013) Characterization of an antigenic chitosanase from the cellulolytic fungus Chaetomium globosum. Medical Mycology, 51: 290-299. 
Purokivi M, Hirvonen MR, Randell J, Roponen M, Meklin T, Nevalainen A, Husman T, Tukiainen H (2001) Changes in pro-inflammatory cytokines in association with exposure to moisture-damaged building microbes. European Journal of Epidemiology, 18: 951-958.

Qin J, Zhang Y, Gao J, Bai M, Yang S, Laatsch H, Zhang A (2009) Bioactive metabolites produced by Chaetomium globosum, an endophytic fungus isolated from Ginkgo biloba. Bioorganic and Medicinal Chemistry Letters, 19: 1572-1574.

Quansah R, Jaakkola MS, Hugg TT, Heikkinen SA, Jaakkola JJ (2012) Residential dampness and molds and the risk of developing asthma: a systematic review and meta-analysis. PLOS ONE, 7: e47526.

Rahbaek L, Sperry S, Frisvad J, Larsen T. 2003. PC-2, LL-P888gamma and some novel analogue $\alpha$-pyrones from Penicillium nordicum, $P$. verrucosum and $P$. olsonii. Biochem Syst Ecol 31:313-317.

Rand TG, DiPenta J, Robbins C, Miller JD (2011) Effects of low molecular weight fungal compounds on inflammatory gene transcription and expression in mouse alveolar macrophages. Chemico-Biological Interactions, 190:139-147.

Rand TG, DiPenta J, Robbins C, Miller JD (2011) Inflammation associated gene modulation in mouse alveolar macrophages induced by low molecular weight compounds from fungi associated with damp building environments. Chemico-Biological Interactions, 190: 139-147.

Rand TG, Flemming J, Giles S, Miller JD, Puniani E (2005) Inflammatory and cytotoxic responses in mouse lungs exposed to purified toxins from building isolated Penicillium brevicompactum Dierckx and P. chrysogenum Thom. Toxicological Science, 87: 213-222.

Rand TG, Flemming J, Miller JD, Womiloju T (2006) Comparison of the inflammatory responses in mouse lungs exposed to atranones A and C from Stachybotrys chartarum. Journal of Toxicologogy and Environmental Health, Part A, 69: 1239-1251.

Rand TG, Robbins C, Rajaraman D, Sun M, Miller JD (2013) Induction of Dectin-1 and asthmaassociated signal transduction pathways in RAW 264.7 cells by a triple-helical $(1,3)-\beta$-D glucan, curdlan. Archives of Toxicology, 87: 1841-1850.

Rand TG, Sun M, Gilyan A, Downey J, Miller JD (2010) Dectin-1 and inflammation-associated gene transcription and expression in mouse lungs by a toxic $(1,3)$-beta-D- glucan. Archives of Toxicology 84: 205-220.

Raper RB and Thom C (1949) A manual of the penicillia. Baltimore: Williams \& Wilkins. pp. 875.

Rasmussen R, Storm I, Rasmussen P, Smedsgaard J, Nielsen KF (2010) Multi-mycotoxin analysis of maize silage by LC-MS/MS. Analytical and Bioanalytical Chemistry, 379: 765-776. 
Reboux G, Piarroux R, Mauny F, Madroszyk A, Millon L, Bardonnet K, Dalphin JC (2001) Role of molds in farmer's lung disease in Eastern France. American Journal of Respiratory Critical Care Medicine, 163: 1534-1539.

Reino J, Guerro R, Hernandez R, Collado I (2008) Secondary metabolites from species of the biocontrol agent Trichoderma. Phytochemistry Reviews, 7: 89-123.

Richard J, Plattner R, Mary J, Liska S (1999) The occurrence of Ochratoxin A in dust collected from a problem household. Mycopathologia, 146: 99-103.

Riche C and Pascard-Billy C (1975) The crystal and molecular structure of phomenone. Acta Crystallographica Section B, 31:1 476-1477.

Rodel T and Gerlach H (1997) Enantioselective synthesis of $(R)-(+)$-pulvilloric acid. European Journal of Organic Chemistry, 197: 213-216.

Safe S and Taylor A (1972) Sporidesmins. 8. Ovine ill-thrift in NovaScotia. 3. The characterisation of chetomin a toxic metabolite of Chaetomium cochliodes and Chaetomium globosum. Journal of the Chemical Society Perkin Trans 1: 472-479.

Sakabe N, Goto T, Hirata Y (1977) Structure of citreoviridin, a mycotoxin produced by Penicillium citreo-viride. Tetrahedron, 33: 3077-3081.

Sakamoto T, Urisu A, Yamada M, Y, Tanaka K, Torii S (1989) Studies on the osmophilic fungus Wallemia sebi as an allergen evaluated by skin prick test and radioallergosorbent test. International Archives of Allergy and Applied Immunology, 90: 368-372.

Samson RA, Houbraken J, Thrane U, Frisvad JC, Andersen B (2010) Food and indoor fungi. Utrecht: CBS-KNAW Fungal Biodiversity Centre. pp. 390.

Salares V, Hinde C, Miller JD (2009) Analysis of settled dust in homes and fungal glucan in air particulate collected during HEPA vacuuming. Indoor Built Environment, 18:485-491. Samuels GJ (1996) Trichoderma: a review of biology and systematics of the genus. Mycological Research, 100: 923-935.

Samuels GJ, Dodd S, Lu B, Petrini O, Schroers H, Druzhinina I (2006) The Trichoderma koningii aggregate species. Studies in Mycology, 56: 67-133.

Scherlach K, Boettger D, Remme N, Hertweck C (2010) The chemistry and biology of cytochalasans. Natural Product Reports, 27:869-886.

Scott J, Untereiner WA, Wong B, Strauss NA, Malloch D (2004) Genotypic variation in Penicillium chrysogenum from indoor environments. Mycologia, 96: 1095-1105. 
Sekita S, Yoshihira K, Natori S, Kuwano H (1973) Structures of chaetoglobosin A and B, cytotoxic metabolites of Chaetomium globosum. Tetrahedron Letters, 1973: 2109-2112.

Sekita S, Yoshihira K, Natori S, Kuwano H (1976) Structures of chaetoglobosins C, chaetoglobosin D, chaetoglobosin E, chaetoglobosin F, cytotoxic indol-3-yl cytochalasans from Chaetomium globosum. Tetrahedron Letters, 1976: 1351-1354.

Sekita S, Yoshihira K, Natori S, Udagawa S, Muroi T, Sugiyamha Y, Kurata H, Umeda M (1981) Mycotoxin production by Chaetomium spp. and related fungi. Canadian Journal of Microbiology, 27: 766-772.

Shi C, Smith M, Miller JD (2011) Characterization of human antigenic proteins SchS21 and SchS34 from Stachybotrys chartarum. International Archives of Allergy and Applied Immunology, 155: 74-85.

Shi C and Miller JD (2011) Characterization of the $41 \mathrm{kDa}$ allergen Asp v 13, a subtilisin-like serine protease from Aspergillus versicolor. Molecular Immunology, 48: 1827-1834.

Shiomi K, Uchida R, Inokoshi J, Tanaka H, Iwai Y, Omura S (1996) Andrastins A-C, new protein farnesyltransferase inhibitors, produced by Penicillium sp. FO-3929. Tetrahedron Letters, 37: $1265-1268$.

Shirota O, Pathak V, Hossain CF, Sekita S, Takatori K, Satake M (1997) Structural elucidation of trichotetronines: polyketides possessing a bicycle [2.2.2] octane skeleton with a tetronic acid moiety isolated from Trichoderma sp. Journal of the Chemical Society, Perkin Transactions 1, 20: 2961-2964.

Silva M, Furtado N, Pupo M, Fonseca M, Said S, da Silva Filho A, Bastos J (2004) Antibacterial activity from Penicillium corylophilum Dierckx. Microbiology Research, 159: 317-322.

Sivithamparam K and Ghisalberti E (1998) Secondary metabolism in Trichoderma and Gliocladium. In: Trichoderma and Gliocladium: Basic Biology, Taxonomy and Genetics, Volume 1. Taylor and Francis, Brisol, PA.

Simon-Nobbe B, Denk U, Pöll V, Rid R, Breitenbach M (2007) The spectrum of fungal allergy. International Archives of Allergy and Applied Immunology, 145: 58-86.

Simon A, Dunlop R, Ghisalberti E, Sivasithamparam K (1988) Trichoderma koningii produces a pyrone compound with antibiotic properties. Soil Biology and Biochemistry, 20: 263-264.

Staunton J and Weissman KJ (2001) Polyketide biosynthesis: a millennium review. Natural Product Reports, 18: 380-416. 
Slack GJ, Puniani E, Frisvad JC, Samson RA, Miller JD. 2009. Secondary metabolites from Eurotium species, A. calidoustus and A. insuetus common in Canadian homes with a review of their chemistry and biological activities. Mycological Research, 113: 480-490.

Slater GP, Haskins RH, Hogge LR, Nesbitt LR (1967) Metabolic products from a Trichoderma viride. Canadian Journal of Chemistry, 45: 92-96.

Smedsgaard J (1997) Micro-scale extraction procedure for standardized screening of fungal metabolite production in cultures. Journal of Chromatography, 760: 264-270.

Smith J, Berry D (1975) The filamentous fungi. Volume 1. Industrial Mycology. Edward Arnold Ltd.

Sonjak S, Frisvad JC, Gunde-Cimerman N (2005) Comparison of secondary metabolite production by Penicillium crustosum strains, isolated from Arctic and other various ecological niches. FEMS Microbiology Ecology, 53: 51-60.

Souza A, Rodrigues-Filho E, Souza A, Pereira J, Calgarotto A, Maso V, Marangoni S, Da Silva S (2008) Koninginins, phospholipase A2 inhibitors from endophytic fungus Trichoderma koningii. Toxicon, 51: 240-250.

Sugaya K, Koshino H, Hongo Y, Yasunaga K, Onose J, Yoshikawa K, Abe N (2008) The biosynthesis of sorbicilinoids in Trichoderma sp. USF-2690: prospects for the existence of a common precursor to sorbicillinol and 5-epihydroxyvertinolide, a new sorbicillinoid member. Tetrahedron Letters, 49: 654-657.

Sumarah MW and Miller JD (2009) Anti-insect metabolites from foliar fungal endophytes of conifer trees. Natural Products Communications, 4: 1497-1504.

Sun C, Pang R, Zhang H, Yang M (2005) Design, synthesis, and biological evaluation of novel 4-hydroxypyrone derivatives as HIV-1 protease inhibitors. Bioorganic \& Medicinal Chemistry Letters, 15: 3257-3262.

Sy-Cordero A, Pearce C, Oberlies N (2012) Revisiting the enniatins: a review of their isolation, biosynthesis, structure determination and biological activities. Journal of Antibiotics (Tokyo), 65: 541-549.

Takahashi T (1997) Airborne fungal colony-forming unites in outdoor and indoor environments in Yokohama, Japan. Mycopathologia, 139: 23-33.

Takahashi M, Koyama K, Natori S (1990) Four new azaphilones from Chaetomium globosum var. flavo-viridae. Chemical and Pharmaceutical Bulletin, 38: 625-628.

Takahashi I, Maruta R, Ando K, Yoshida M, Iwasaki T, Kanazawa J, Okabe M, Tamaoki T (1993) UCA1064-B, a new antitumor antibiotic isolated from Wallemia sebi: production, isolation and structural determination, Journal of Antibiotics (Tokyo), 46: 1312-1314. 
Takaiwa A and Yamashita K (1983) Stereochemistry of (-)-vertinolide. Agricultural and Biological Chemistry, 47: 429-430.

Tamura A, Kotani, H. and Naruto S (1975) Trichoviridin and dermadin from Trichoderma sp. TK-1. Journal of Antibiotics, 28: 161-162.

Täubel M, Sulyok M, Vishwanath V, Bloom E, Turunen M, Järvi K, Kauhanen E, Krska R, Hyvärinen A, Larsson L, Nevalainen A (2011) Co-occurrence of toxic bacterial and fungal secondary metabolites in moisture-damaged indoor environments. Indoor Air 21: 368-375.

Taylor A (1985) Some aspects of the chemistry and biology of the genus Hypocrea and its anamorphs, Trichoderma and Gliocladium. Proceedings of the Nova Scotian Institute of Science, 36: $27-58$.

Thrane U, Poulsen SB, Nirenberg HI, Lieckfeldt E (2001) Identification of Trichoderma strains by image analysis of HPLC chromatograms. FEMS Microbiology Letters 203: 249-255.

Tomoda H, Matsushima C, Tabata N, Namatame I, Tanaka H, Bamberger MJ, Arai H, Fukazawa M, Inoue K, Omura S (1999) Structure specific inhibition of cholesteryl ester transfer protein by azaphilones. Journal of Antibiotics (Tokyo), 52: 160-170.

Torssell K (1987) Natural product chemistry. New York, John Wiley and Sons Ltd.

Trifonov L, Bieri J, Prewo R, Dreding S, Rast D, Hoesh L (1982) The constitution of vertinolide, a new derivative of tetronic acid, produced by Verticillium intertextum. Tetrahedron 3: 397-403.

Trifonov L, Hilpert H, Floersheim P, Dreiding A, Rast D, Skrivanova R, Hoesch (1986) Bisvertinols: a new group of dimeric vertinoids from Verticillium intertextum. Tetrahedron, 42 : 3157-3179.

Trown PW (1968) Antiviral activity of N, N'-dimethyl-epidithiapiperazinedione, a synthetic compound related to the gliotoxins, LLS88alpha and beta, chetomin and the sporidesmins. Biochemical and Biophysical Research Communications, 8:402-407.

Tuomi T, Reijula K, Johnsson T, Hemminki K, Hintikka E-L, Lindroos O, Kalso S, KoukilaKahkola P, Mussalo-Rauhamaa H, Haahtela T (2000) Mycotoxins in crude building materials from water-damaged buildings. Applied Environmental Microbiology, 66: 1899-1904.

Twine S, Mykytczuk N, Petit M, Tremblay T, Conlan W, Kelly J (2005) Francisella tularensis Proteome: Low Levels of ASB-14 Facilitate the Visualization of Membrane Proteins in Total Protein Extracts. Journal of Proteome Research, 4: 1848-1854. 
Udagawa S, Muroi T, Kurata H, Sekita S, Yoshihira K, Natori S (1978) The production of chaetoglobosins, sterigmatocystin, O-methylsterigmatocystin, and chaetocin by Chaetomium spp. and related fungi. Canadian Journal of Microbiology, 25: 170-177.

Umeda M, Ohtsubo K, Saito M, Sekita S, Yoshira K, Natori S, Udagawa S, Sakabe, Kurata H (1975) Cytotoxicity of new cytochalasans from Chaetomium globosum. Experientia, 31: 435438.

Unoura K, Miyazaki Y, Sumi Y, Tamaoka M, Sugita T, Inase N (2011) Identification of fungal DNA in BALF from patients with home-related hypersensitivity pneumonitis. Respiratory Medicine, 105: 1696-1703.

Vinale F, Marra R, Scala F, Ghisalberti EL, Lorito M, Sivasithamparam K (2006) Major secondary metabolites produced by two commercial Trichoderma strains active against different phytopathogens. Letters in Applied Microbiology, 43: 143-148.

Vishwanath V, Sulyok M, Labuda R, Bicker W, Krska R (2009) Simultaneous determination of 186 fungal and bacterial metabolites in indoor matrices by liquid chromatography tandem mass spectrometry. Analytical and Bioanalytical Chemistry, 395: 1355-1372.

Von Arx JA, Guarro J, Figueras MJ (1986) The ascomycete genus Chaetomium. Nova Hedwig, 84: 1-162.

Wang Y, Xu L, Ren W, Zhao D, Zhu Y, Wu X (2012) Bioactive metabolites from Chaetomium globosum L18, an endophytic fungus in the medicinal plant Curcuma wenyujin. Phytomedicine, 19: 364-368.

Washida K, Abe N, Sugiyama Y, Hirota A (2009) Novel secondary metabolites, spirosorbicillinols A, B and C, from a fungus. Bioscience, Biotechnology and Biochemistry, 73: 1355-1361.

Watling R (2003) Fungi. The Natural History Museum. London.

Wheeler K, Hocking A, Pitt JI (1988) Effects of temperature and water activity on germination and growth of Wallemia sebi. Transactions of the British Mycological Society, 90: 365-368.

Wicklow D (1981) Interference competition and the organization of fungal communities. In: Wicklow D, Carroll, G. The fungal community, its organization and role in the ecosystem. Marcel Dekker Inc.

Wilson AM, Luo W, Miller JD (2009) Using human sera to identify a $52 \mathrm{kDa}$ exoantigen of Penicllium chrysogenum and implications of polyphasic taxonomy of anamorphic ascomycetes in the study of allergens. Mycopathologia, 168: 213-226. 
Winter J, Sato M, Sugimoto S, Chiou G, Garg N, Tang Y, Watanabe K (2012) Identification and characterization of the chaetoviridin and chaetomugilin gene cluster in Chaetomium globosum reveal dual functions of an iterative highly reducing polyketide synthase. Journal of the American Chemical Society, 134: 17900-17903.

Wood G, Mann P, Reid W, Moss M (1990) Studies on a toxic metabolite from the mould Wallemia. Food Additives and Contaminants, 7: 69-77.

World Health Organization (2009) WHO guidelines for indoor air quality: dampness and mould. WHO, Regional Office for Europe, DK-2100. Copenhagen, Denmark.

Wrobel J and Ganem B (1983) Total Synthesis of (-)-vertinolide. A general approach to chiral tetronic acids and butenolides from allylic alcohols. Journal of Organic Chemistry, 48: 37613764.

Xu J, Jensen J, Liang Y, Belisle D, Miller JD.(2007) The biology and immogenicity of a $34 \mathrm{kDa}$ antigen of Stachybotrys chartarum sensu latto. International Journal of Biodegradation and Biodeterioration, 60: 308-318.

Xu J, Liang Y, Belisle D, Miller JD (2008) Characterization of monoclonal antibodies to an antigenic protein from Stachybotrys chartarum and its measurement in house dust. Journal of Immunological Methods, 332: 121-128.

$\mathrm{Xu} \mathrm{X}$ and Zhu Y (1995) Total synthesis of koninginin A and its diastereoisomer. Tetrahedron Letters, 36: 9173-9176.

Yasuhide M, Yamada T, Numata A, Tanaka T (2008) Chaetomugilins, new selectively cytotoxic metabolites, produced by a marine fish derived Chaetomium species. Journal of Antibiotics, 61: 615-622.

Zhang D, Li X, Kang J, Choi H, Son B (2007) A new alpha pyrone derivative, 6-[(E)-Hept-1enyl]-alpha-pyrone, with tyrosine inhibitory activity from a marine isolate of the fungus Botryis. Bulletins of the Korean Chemical Society, 28: 887-888.

Zalar P, de Hoog G, Schroers H., Frank J, Gunde-Cimerman N (2005) Taxonomy and phylogeny of the xerophilic genus Wallemia (Wallemiomycetes and Wallemiales, cl. et ord. nov.). Antonie van Leeuwenhoek. 87: 311-328.

Zhang Q, Li H, Zong S, Gao J, Zhang A (2012) Chemical and bioactive diversities of the genus Chaetomium secondary metabolites. Mini-Reviews in Medicinal Chemistry, 12: 127-148.

Zhou H, Qiao K, Gao Z, Meehan M, Li, J, Zhao X, Dorrestein P, Vederas J, Tang Y (2010) Enzymatic synthesis of resorcyclic acid lactones by cooperation of fungal iterative polyketide synthases involved in hypothemycin biosynthesis. Journal of the American Chemical Society, 132: 4530-4531. 
APPENDIX I - ${ }^{1} \mathrm{H}$ and ${ }^{13} \mathrm{C}$ NMR spectra for isolated secondary metabolites 
${ }^{1} \mathrm{H}-\mathrm{NMR}\left(\mathrm{CD}_{3} \mathrm{CN}, 400 \mathrm{MHz}\right)$ spectrum of compound $\mathbf{2 . 1}$

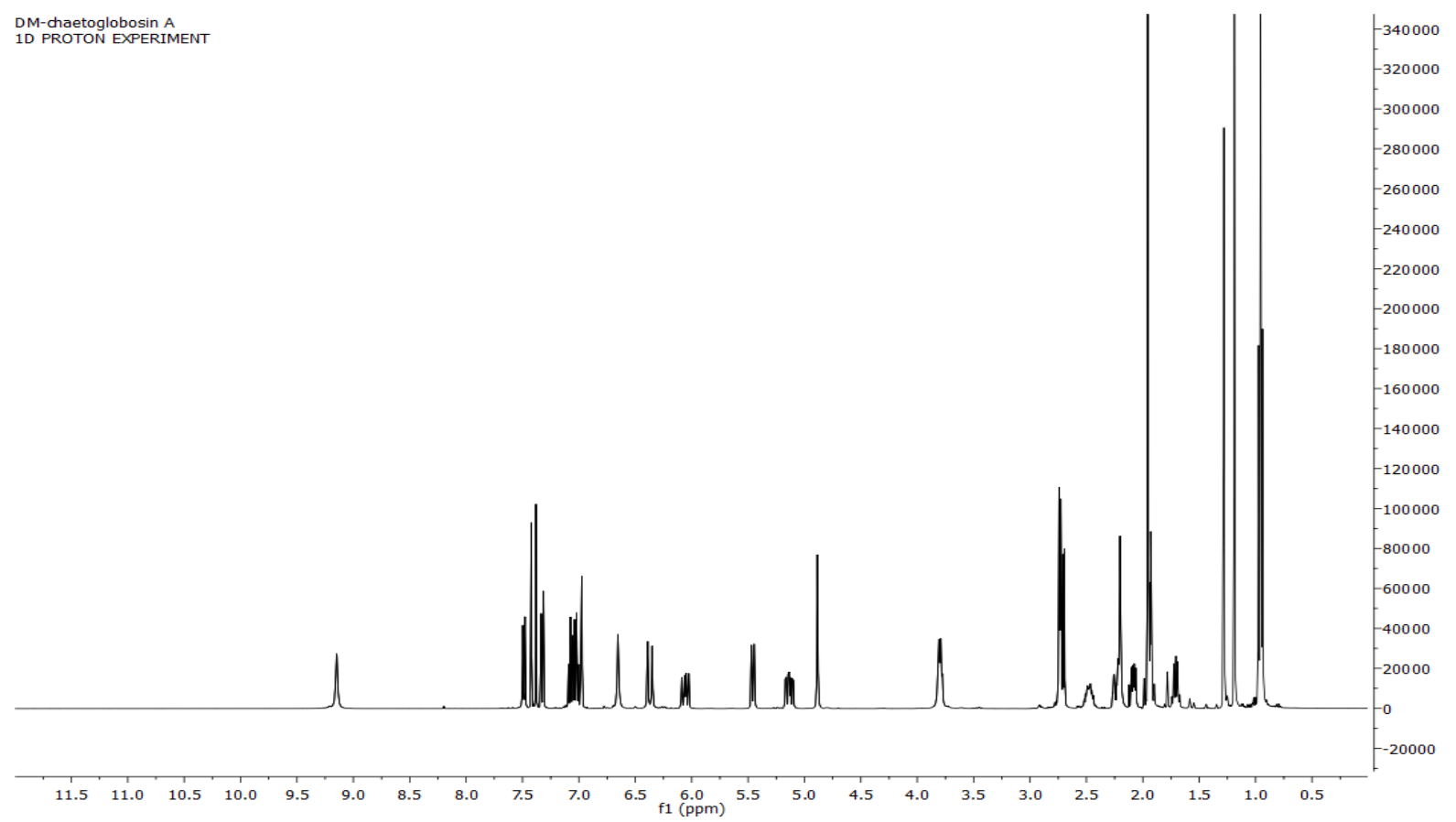

${ }^{13} \mathrm{C}-\mathrm{NMR}\left(\mathrm{CD}_{3} \mathrm{CN}, 100 \mathrm{MHz}\right)$ spectrum of compound $\mathbf{2 . 1}$

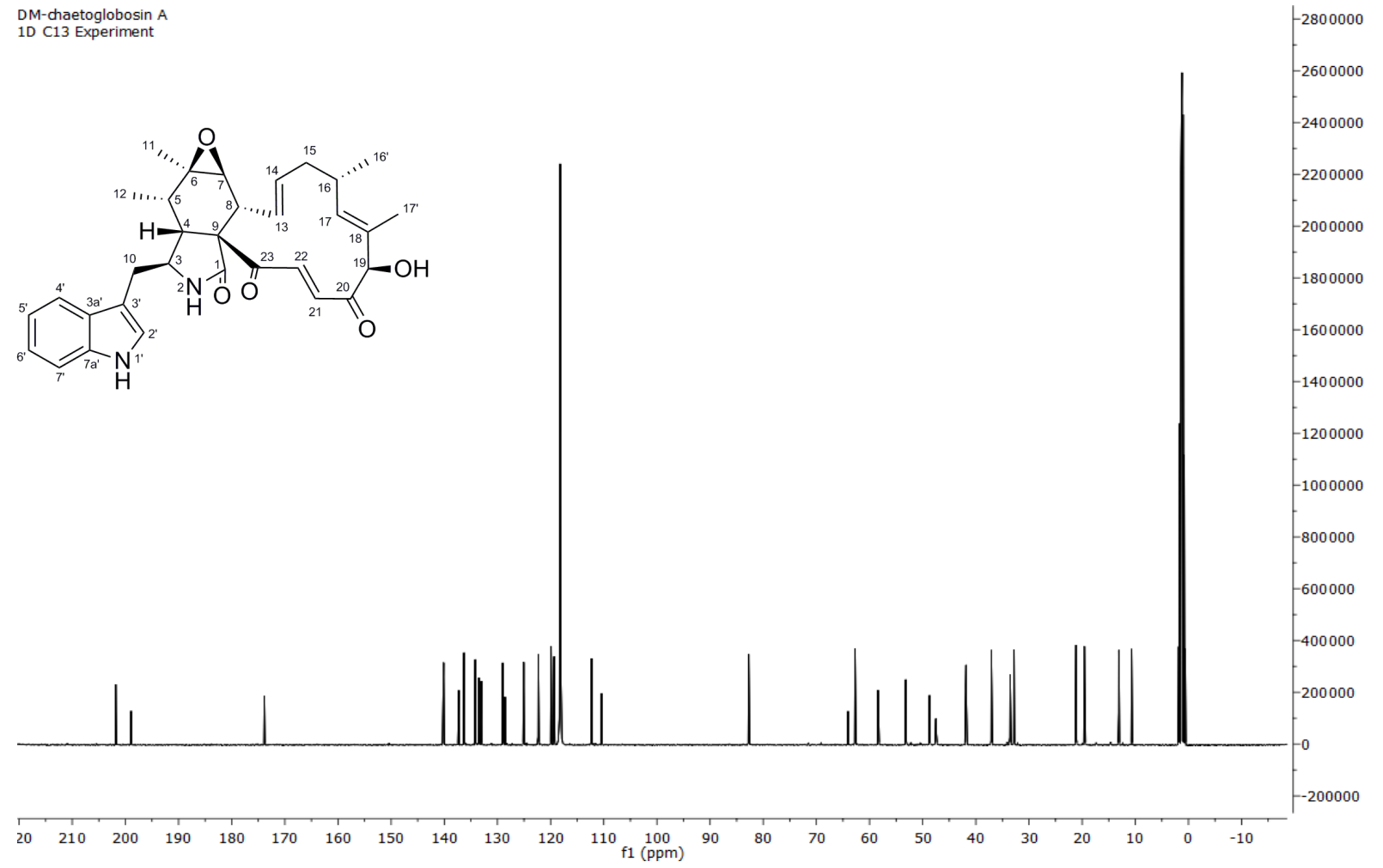


${ }^{1} \mathrm{H}-\mathrm{NMR}\left(\mathrm{CDCl}_{3}, 400 \mathrm{MHz}\right)$ spectrum of compound $\mathbf{2 . 2}$

DM-Chaetoglobosin 530-Sept/Proton

1D PROTON EXPERIMENT

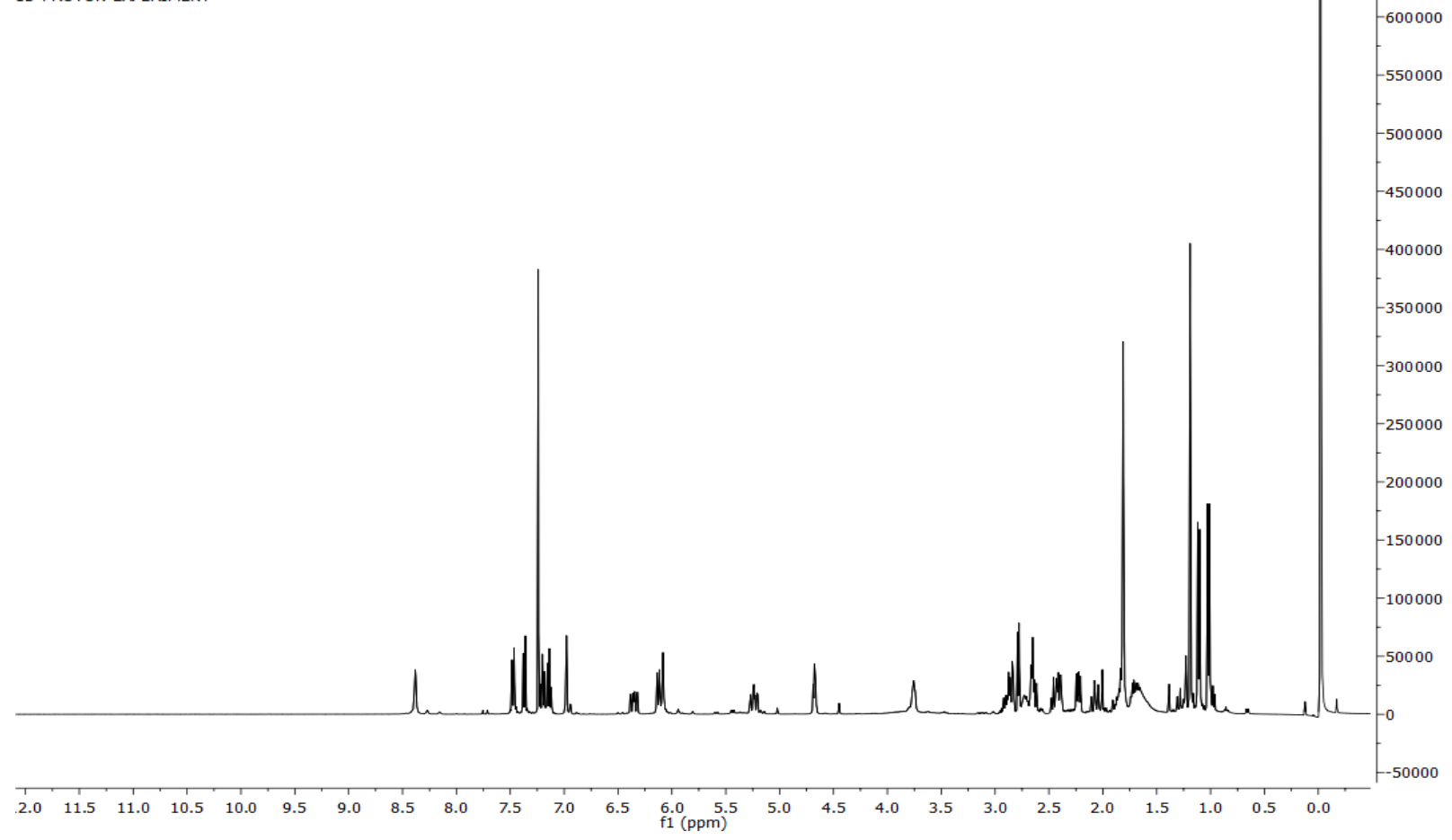

${ }^{13} \mathrm{C}$-NMR $\left(\mathrm{CDCl}_{3}, 100 \mathrm{MHz}\right)$ spectrum of compound $\mathbf{2 . 2}$

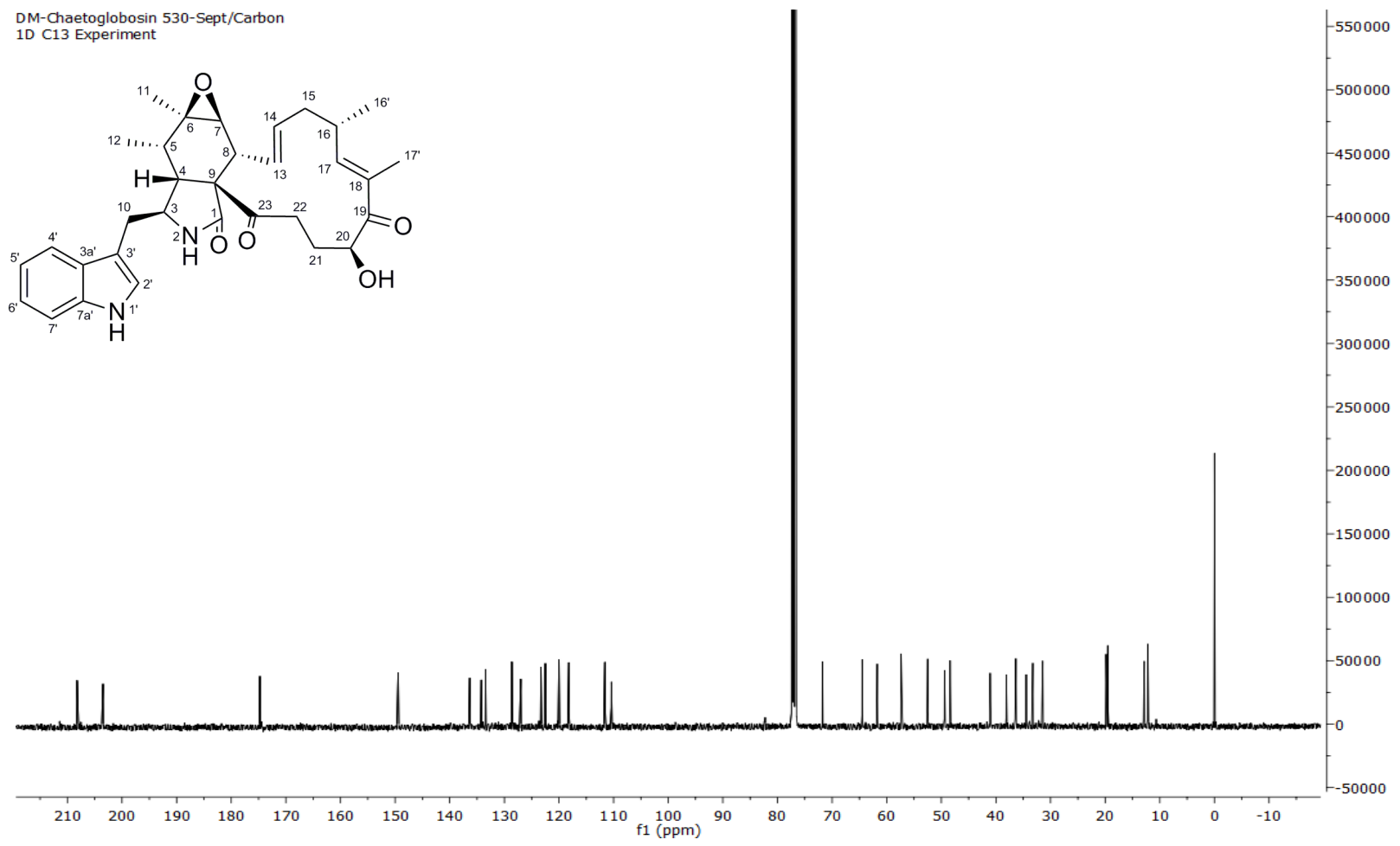


${ }^{1} \mathrm{H}-\mathrm{NMR}\left(\mathrm{CD}_{3} \mathrm{OD}, 400 \mathrm{MHz}\right)$ spectrum of compound $\mathbf{2 . 3}$

$\mathrm{cg}-3-\mathrm{c}$
1D PROTON EXPERIMENT
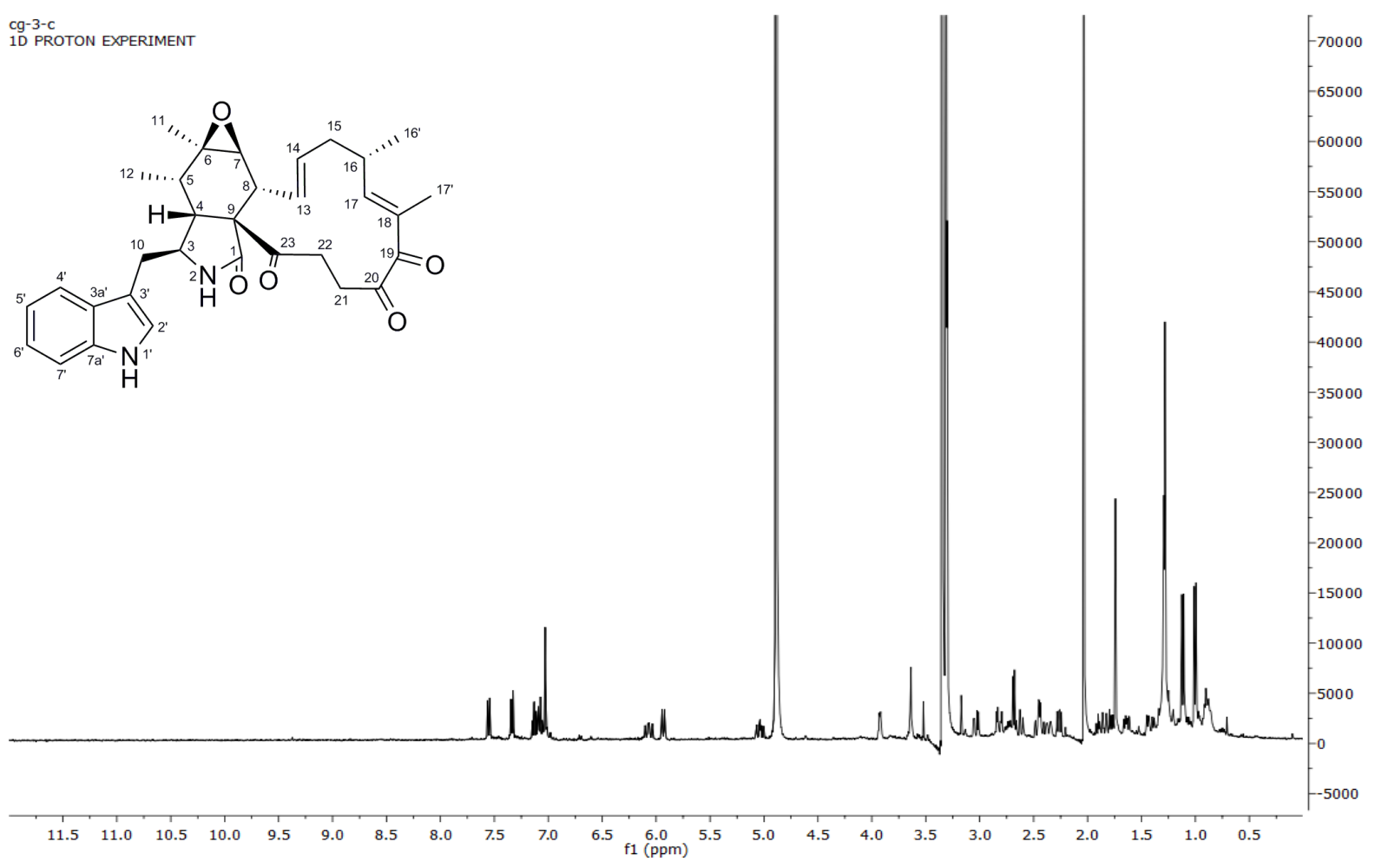
${ }^{1} \mathrm{H}-\mathrm{NMR}\left(\mathrm{CD}_{3} \mathrm{CN}, 400 \mathrm{MHz}\right)$ spectrum of compound $\mathbf{2 . 4}$

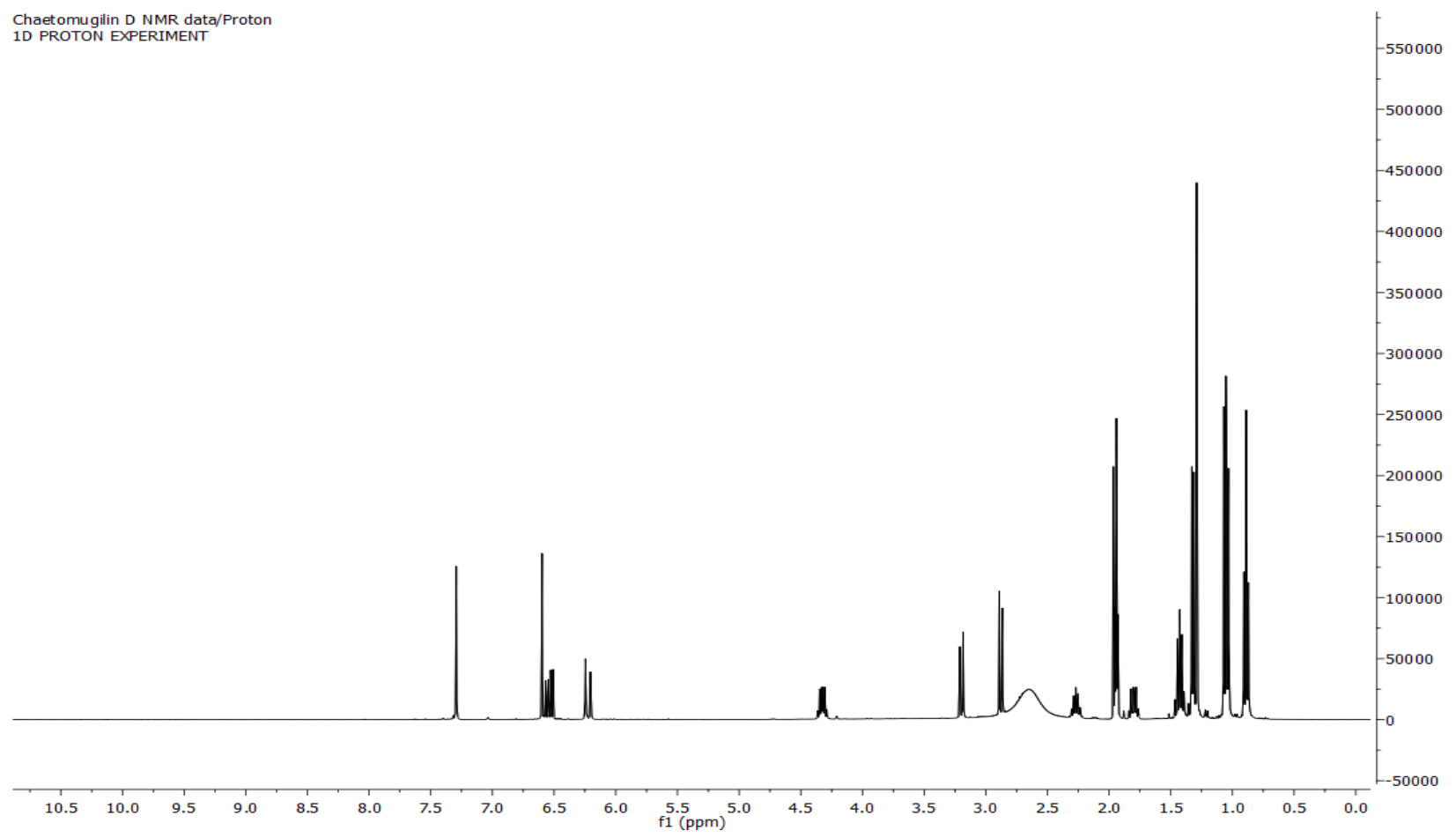

\section{${ }^{13} \mathrm{C}$-NMR $\left(\mathrm{CD}_{3} \mathrm{CN}, 100 \mathrm{MHz}\right)$ spectrum of compound $\mathbf{2 . 4}$}

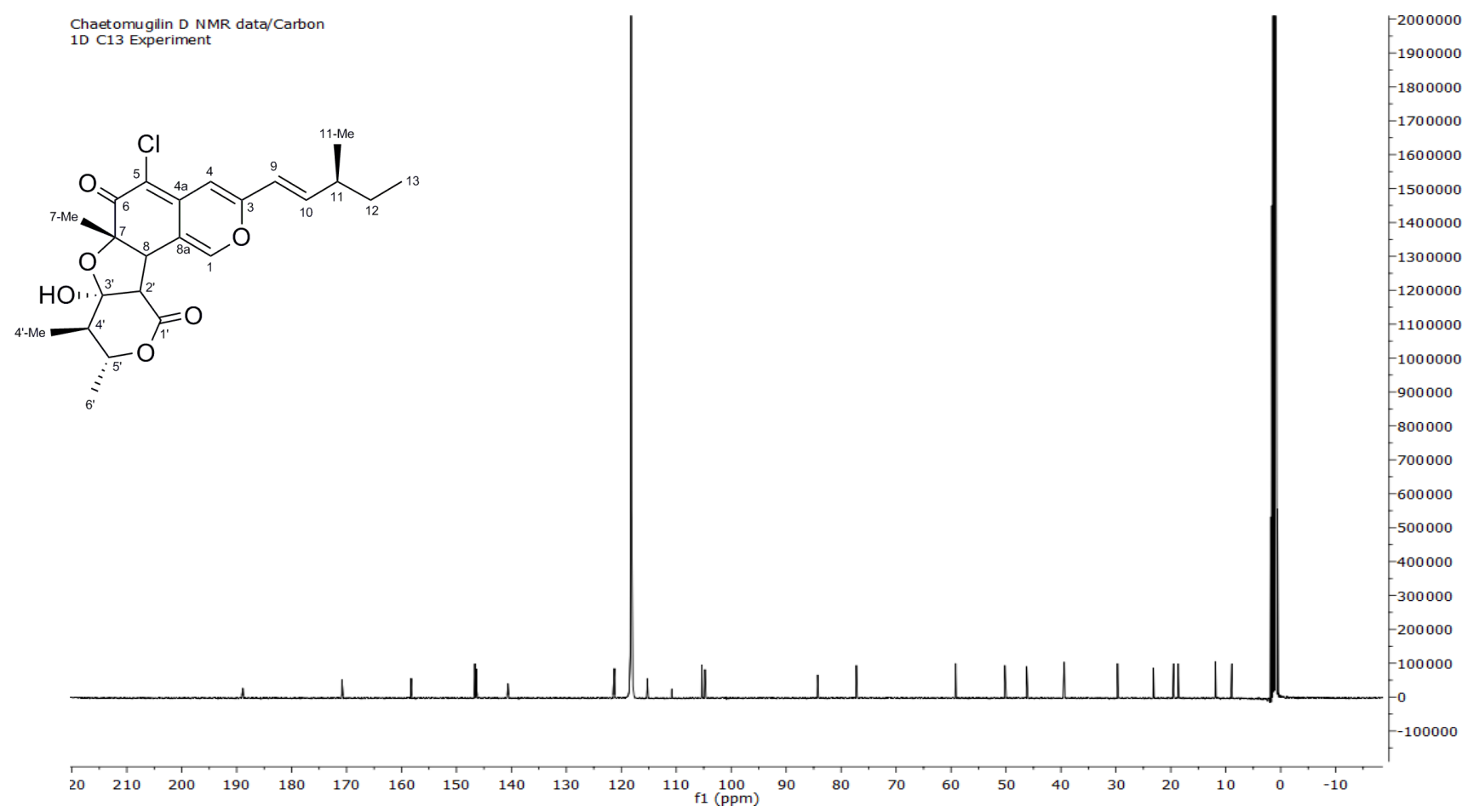


${ }^{1} \mathrm{H}-\mathrm{NMR}\left(\mathrm{CD}_{3} \mathrm{CN}, 400 \mathrm{MHz}\right)$ spectrum of compound $\mathbf{2 . 5}$

DM-c.glob-432-NMR spectra/Proton

1D PROTON EXPERIMENT

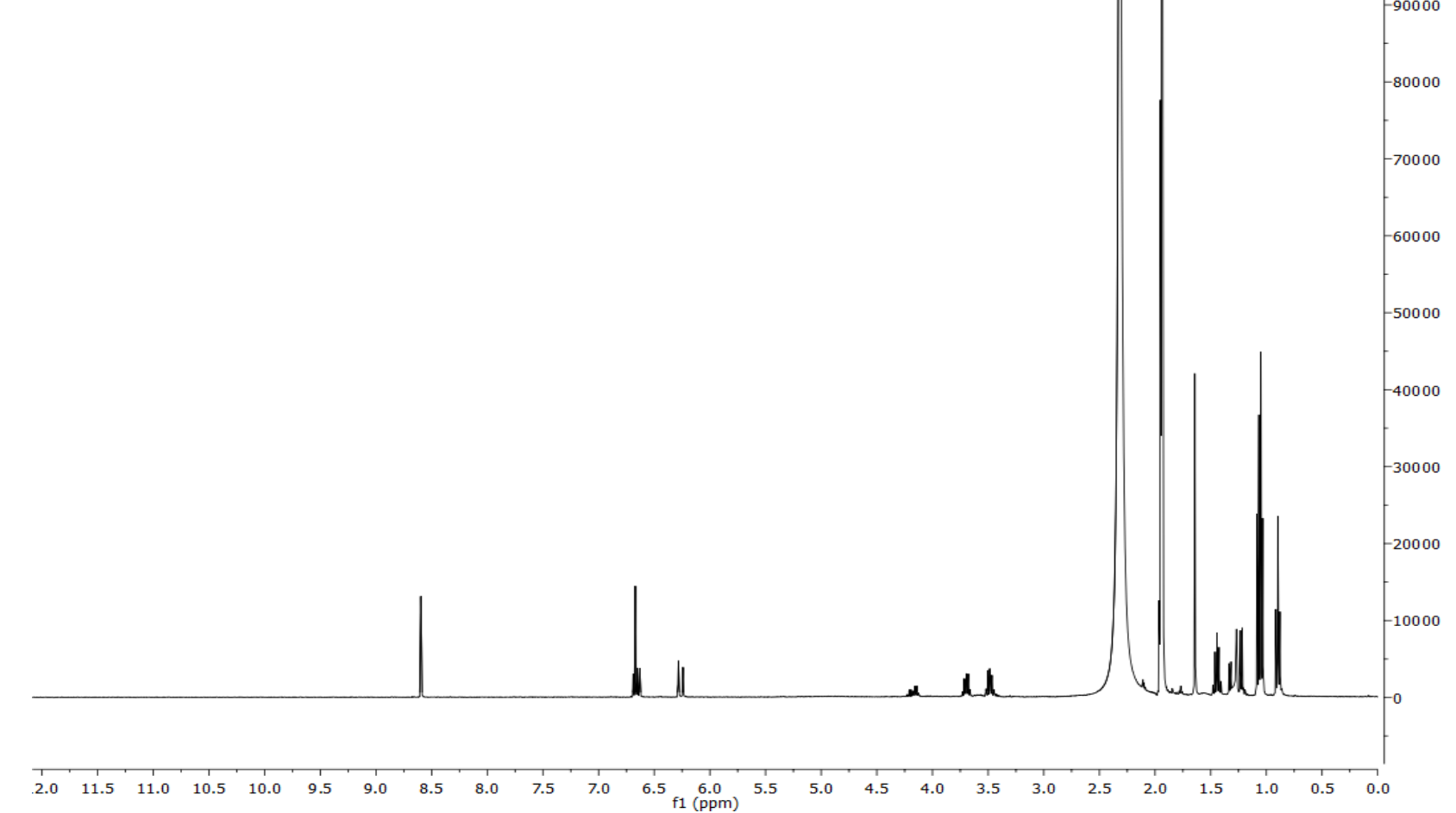

${ }^{13} \mathrm{C}-\mathrm{NMR}\left(\mathrm{CD}_{3} \mathrm{CN}, 100 \mathrm{MHz}\right)$ spectrum of compound $\mathbf{2 . 5}$

Chaetoviridin A-Auqust/Carbon<smiles>CC[C@H](C)/C=C/C1=CC2=C(Cl)C(=O)[C@]3(C)OC(=O)C4=C3C2=C(O1)[C@H]([C@@H](C)O)C4=O</smiles>

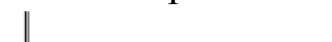

| 
${ }^{1} \mathrm{H}-\mathrm{NMR}\left(\mathrm{CD}_{3} \mathrm{CN}, 400 \mathrm{MHz}\right)$ spectrum of compound $\mathbf{2 . 6}$

DM-red compound carbon/Proton

1D PROTON EXPERIMENT

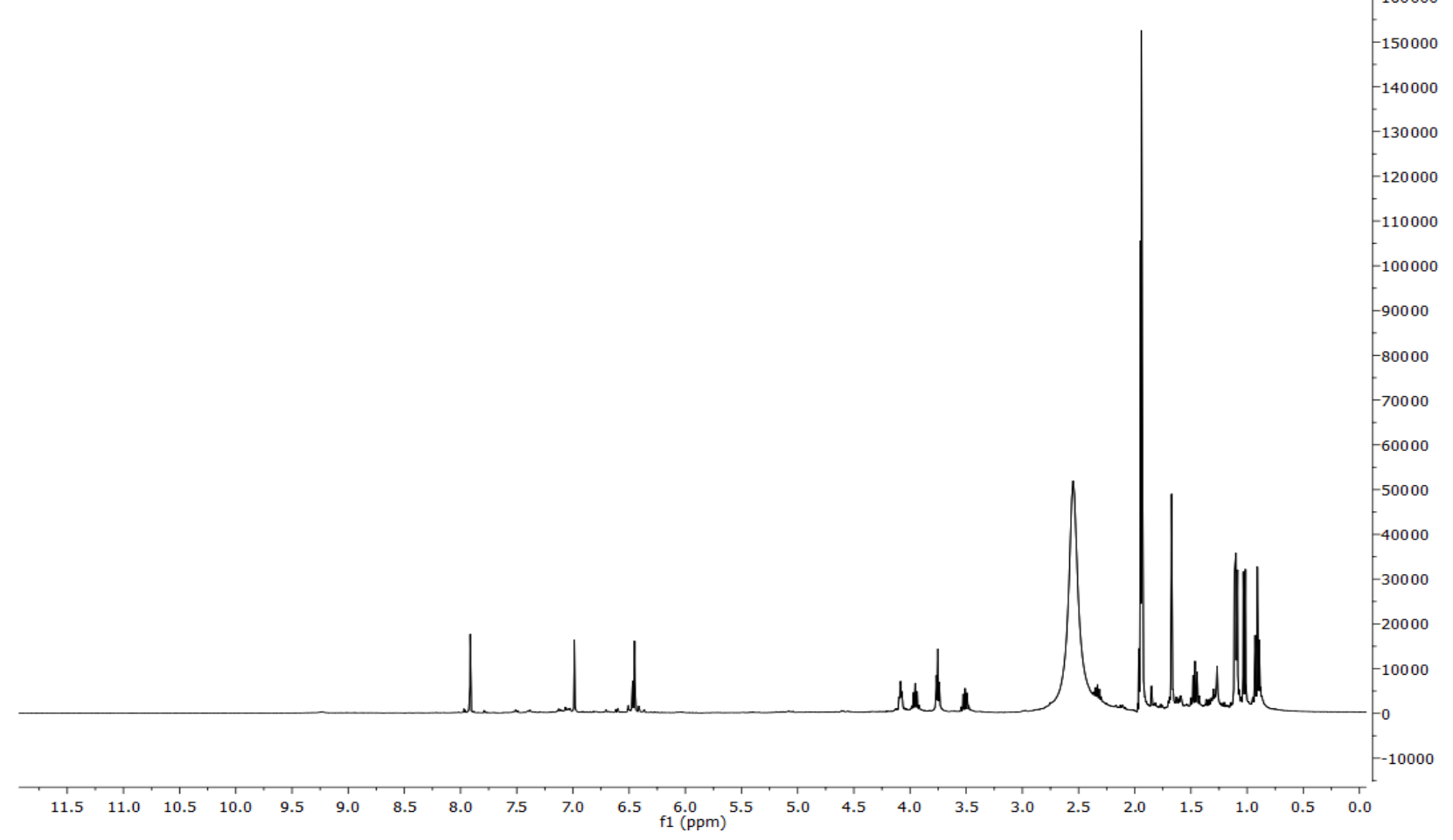

${ }^{13} \mathrm{C}$-NMR $\left(\mathrm{CD}_{3} \mathrm{CN}, 100 \mathrm{MHz}\right)$ spectrum of compound $\mathbf{2 . 6}$

DM-red compound carbon/Carbon
1D C13 Experiment

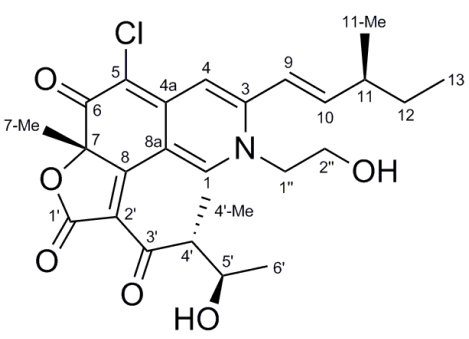

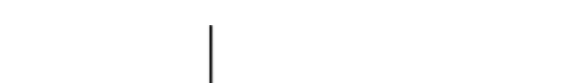


${ }^{1} \mathrm{H}-\mathrm{NMR}\left(\mathrm{CD}_{3} \mathrm{OD}, 400 \mathrm{MHz}\right)$ spectrum of compound $\mathbf{2 . 7}$

DM-499 comp meoh/1

1D PROTON EXPERIMENT

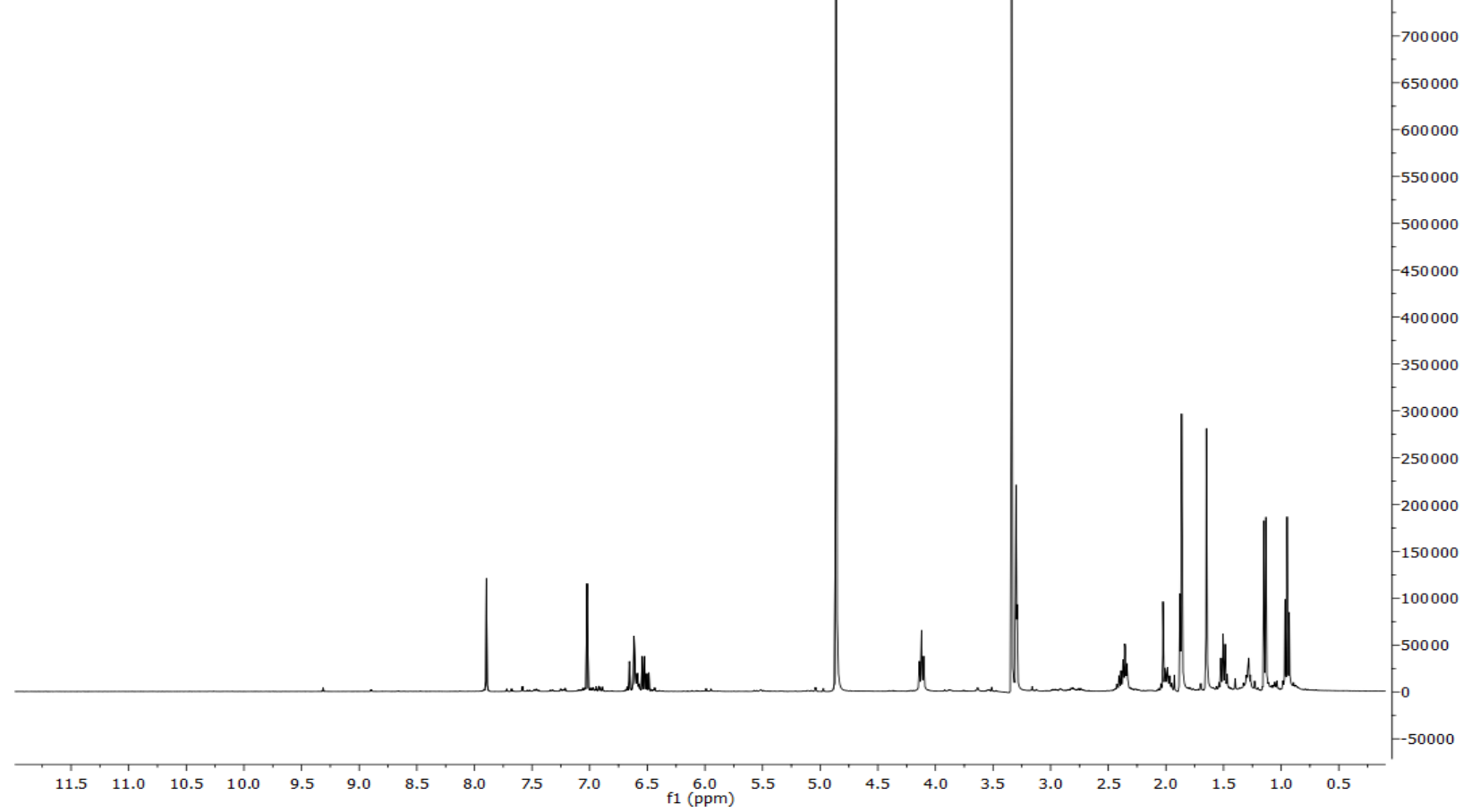

${ }^{13} \mathrm{C}$-NMR $\left(\mathrm{CD}_{3} \mathrm{OD}, 100 \mathrm{MHz}\right)$ spectrum of compound $\mathbf{2 . 7}$

DM-499 comp meoh/
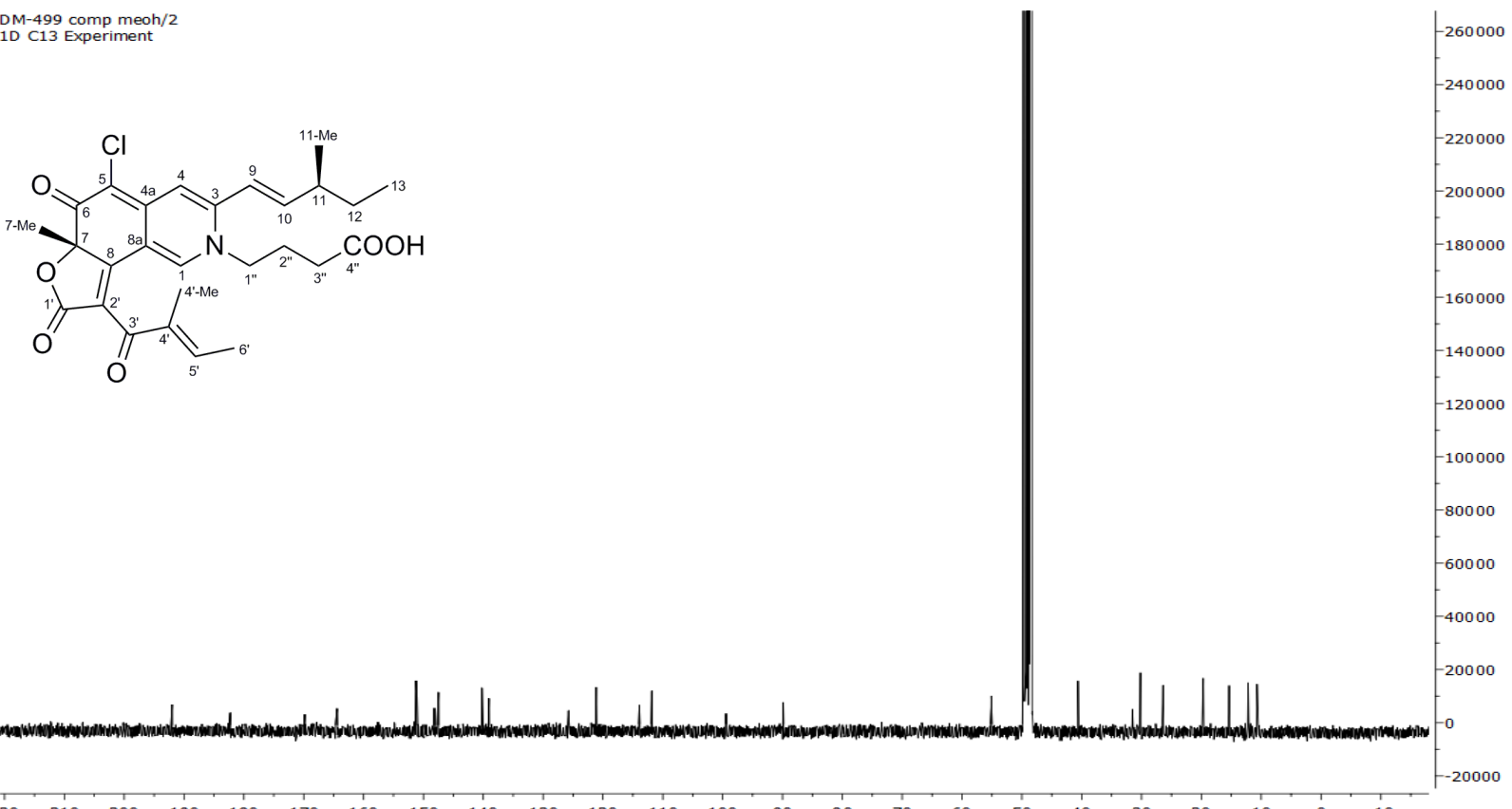

$\begin{array}{llllllllllllllllllllllll}20 & 210 & 200 & 190 & 180 & 170 & 160 & 150 & 140 & 130 & 120 & 110 & 100 & 90 & 80 & 70 & 60 & 50 & 40 & 30 & 20 & 10 & 0 & -10\end{array}$ 


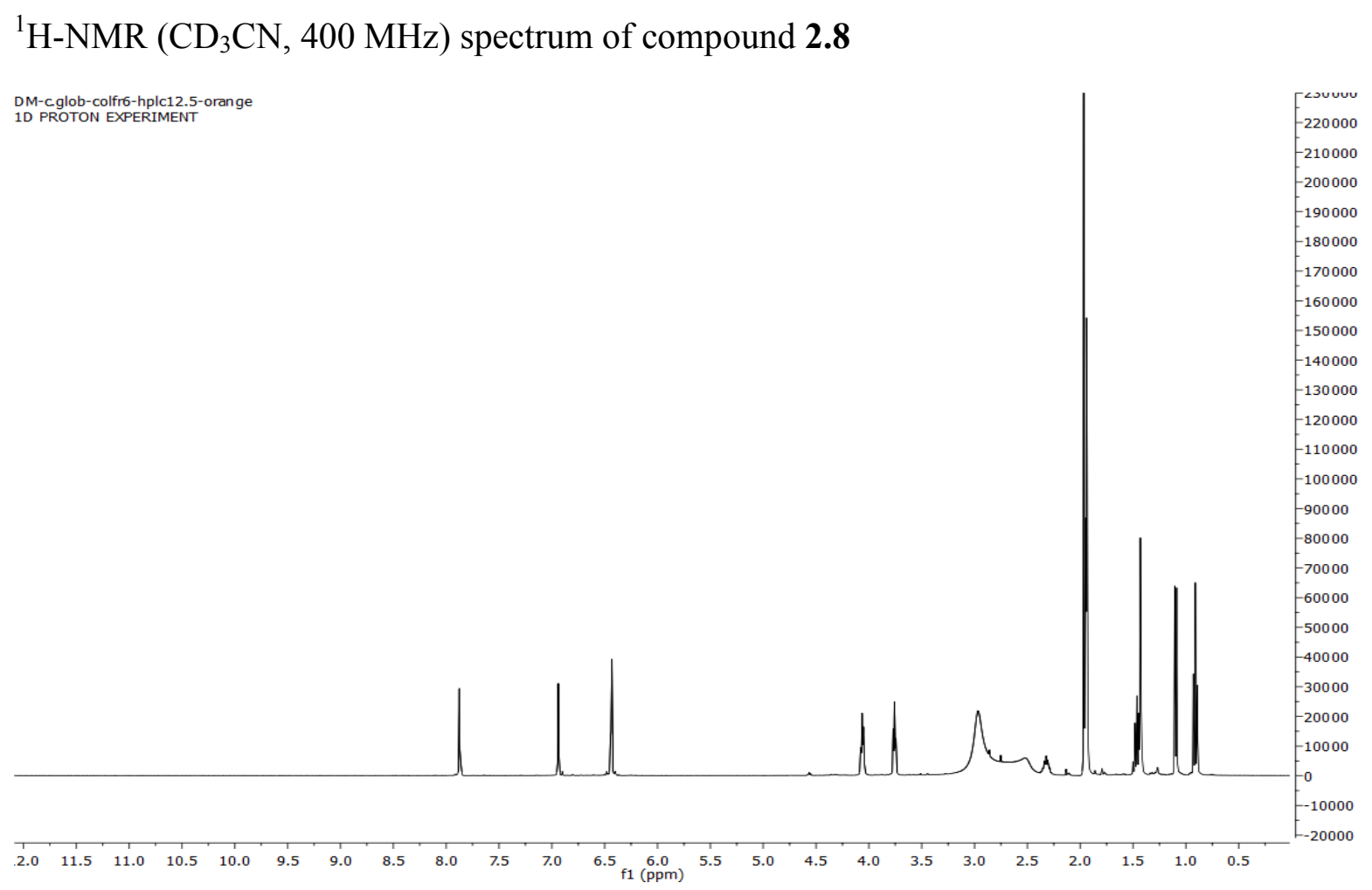

${ }^{13} \mathrm{C}-\mathrm{NMR}\left(\mathrm{CD}_{3} \mathrm{CN}, 100 \mathrm{MHz}\right)$ spectrum of compound $\mathbf{2 . 8}$

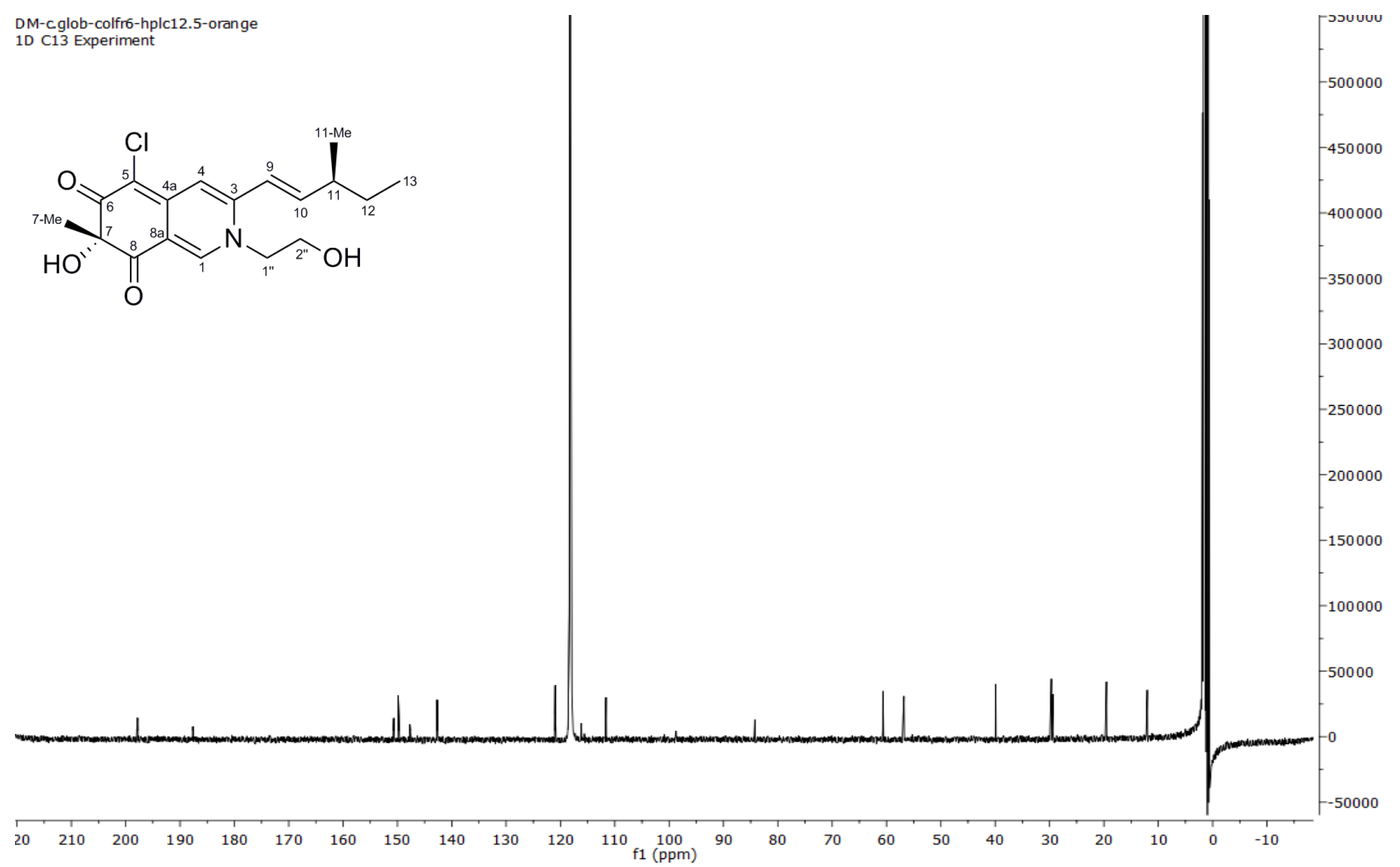




\section{${ }^{1} \mathrm{H}-\mathrm{NMR}\left(\mathrm{CD}_{3} \mathrm{OD}, 700 \mathrm{MHz}\right)$ spectrum of compound $\mathbf{3 . 1}$}

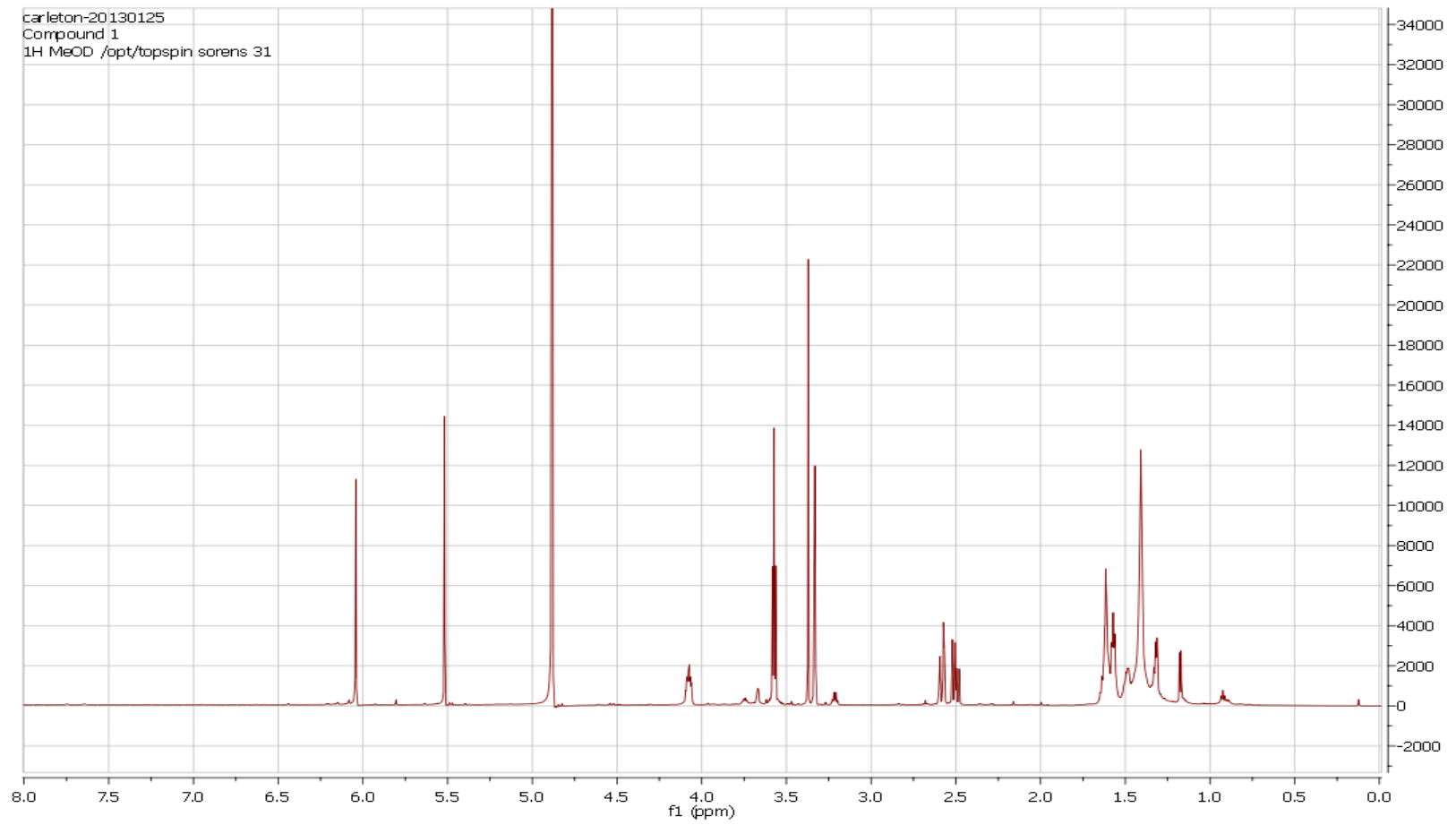

${ }^{13} \mathrm{C}$-NMR $\left(\mathrm{CD}_{3} \mathrm{OD}, 175 \mathrm{MHz}\right)$ spectrum of compound $\mathbf{3 . 1}$

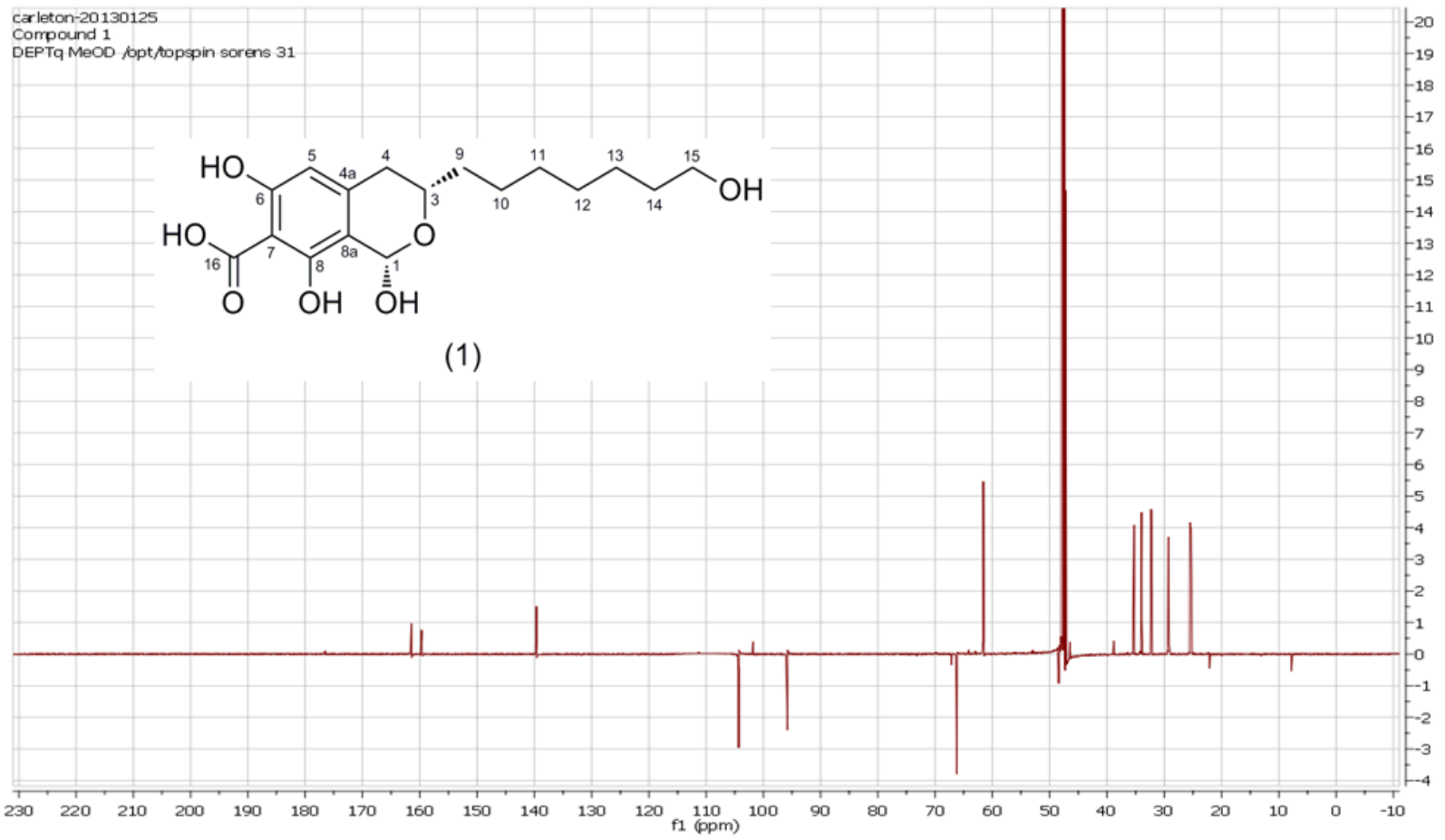


${ }^{1} \mathrm{H}-\mathrm{NMR}\left(\mathrm{CD}_{3} \mathrm{OD}, 700 \mathrm{MHz}\right)$ spectrum of compound $\mathbf{3 . 2}$

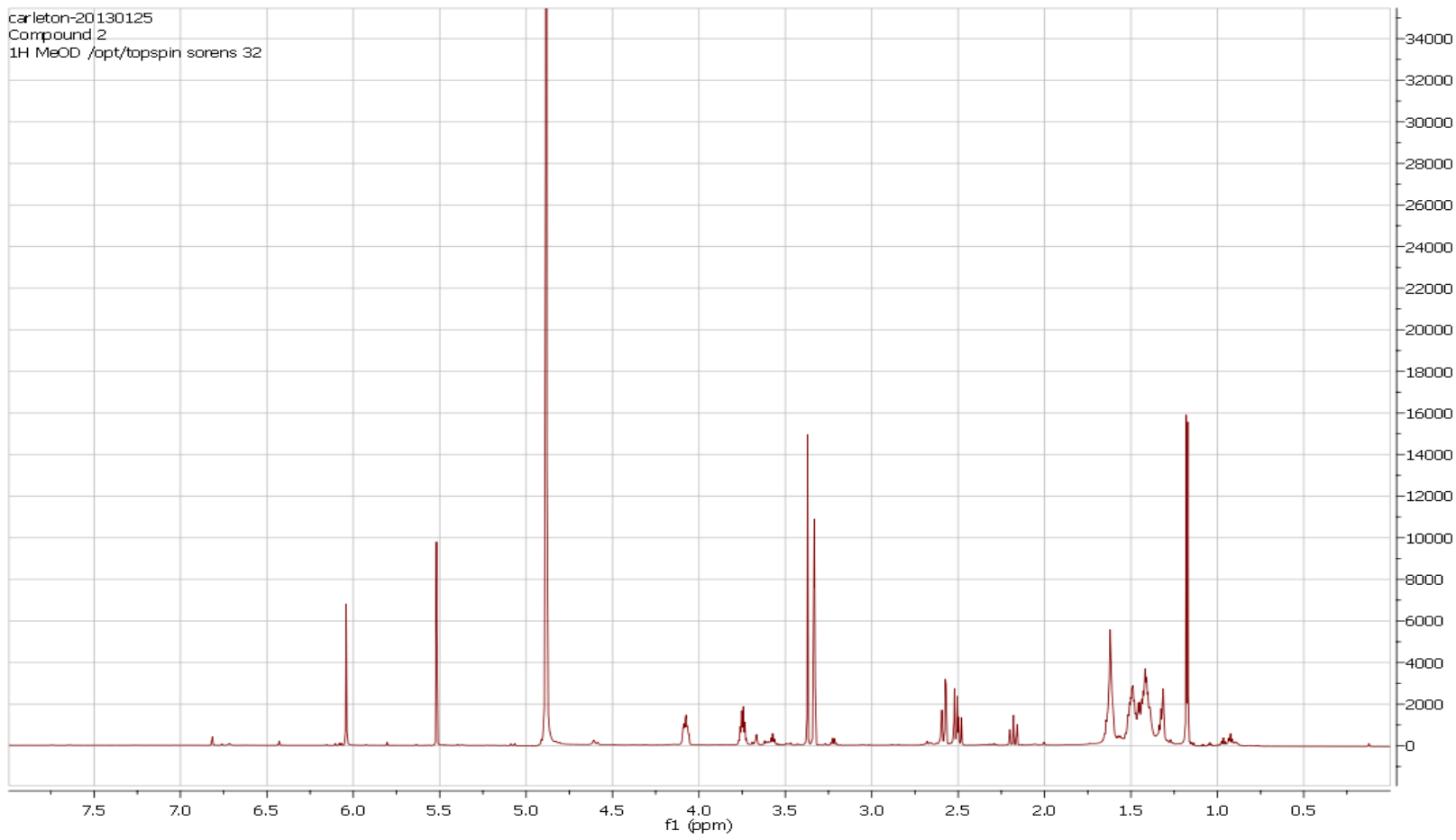

${ }^{13} \mathrm{C}-\mathrm{NMR}\left(\mathrm{CD}_{3} \mathrm{OD}, 175 \mathrm{MHz}\right)$ spectrum of compound $\mathbf{3 . 2}$

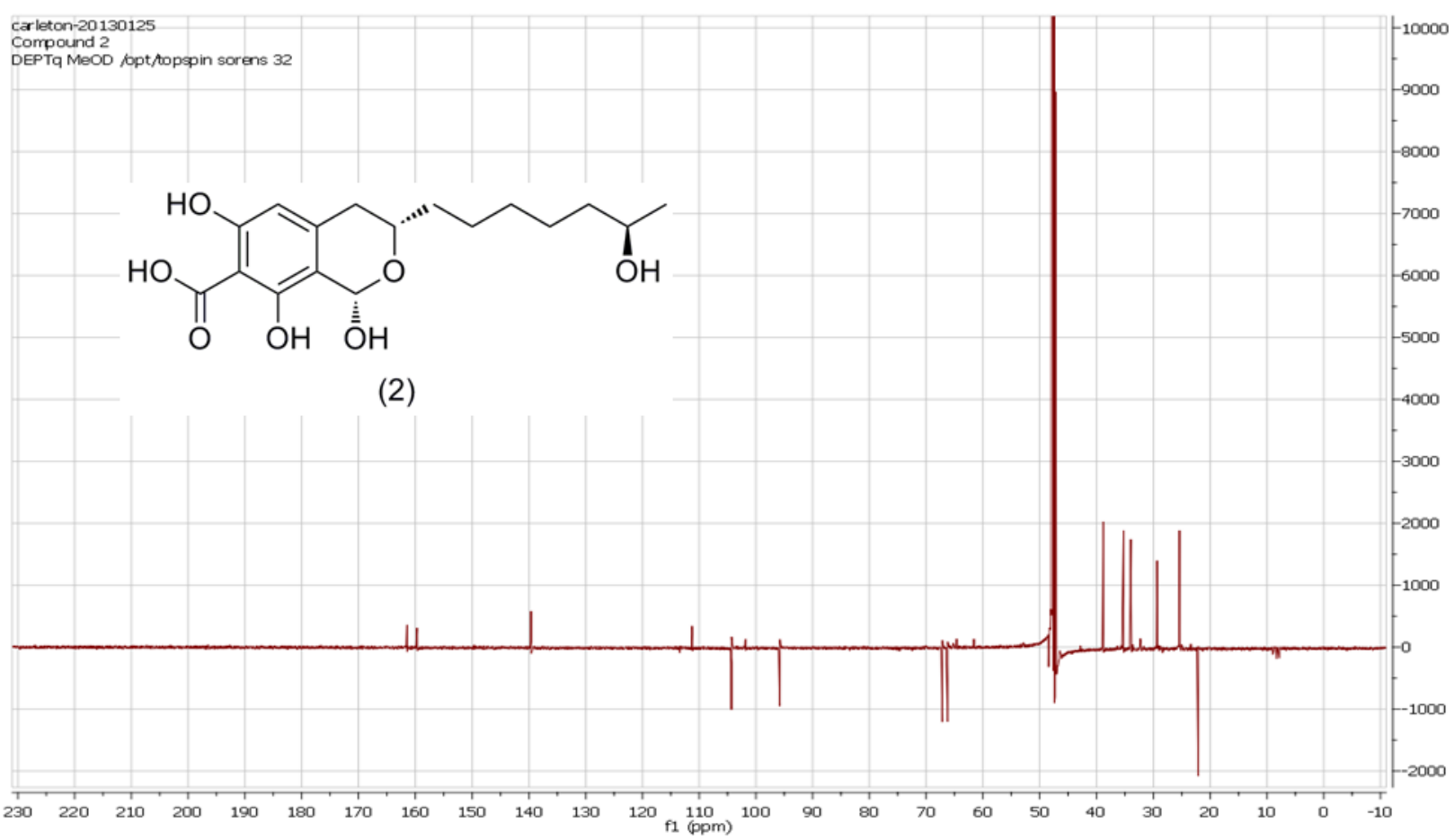




\section{${ }^{1} \mathrm{H}-\mathrm{NMR}\left(\mathrm{CD}_{3} \mathrm{OD}, 700 \mathrm{MHz}\right)$ spectrum of compound $\mathbf{3 . 3}$}

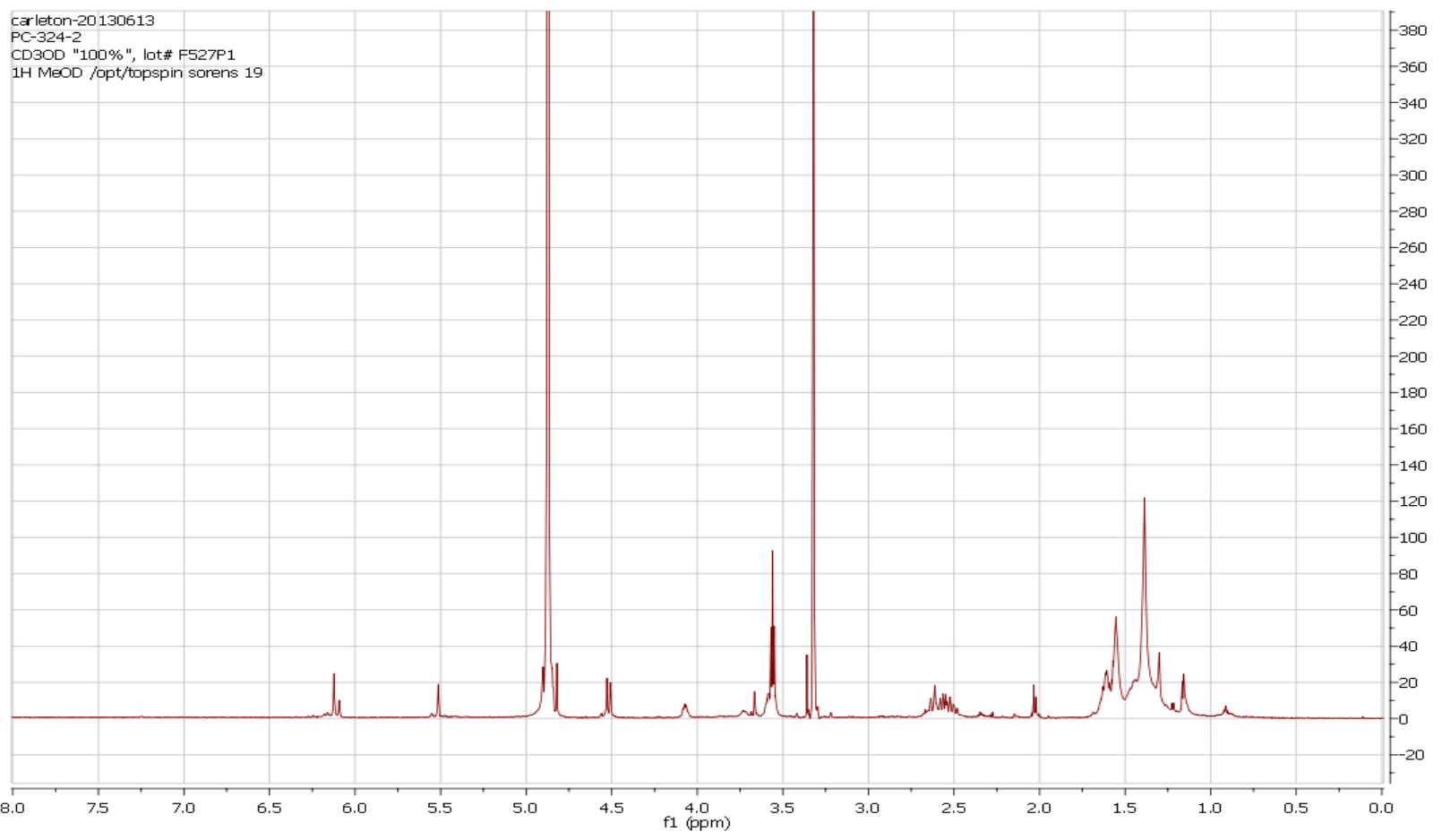

${ }^{13} \mathrm{C}-\mathrm{NMR}\left(\mathrm{CD}_{3} \mathrm{OD}, 175 \mathrm{MHz}\right)$ spectrum of compound $\mathbf{3 . 3}$

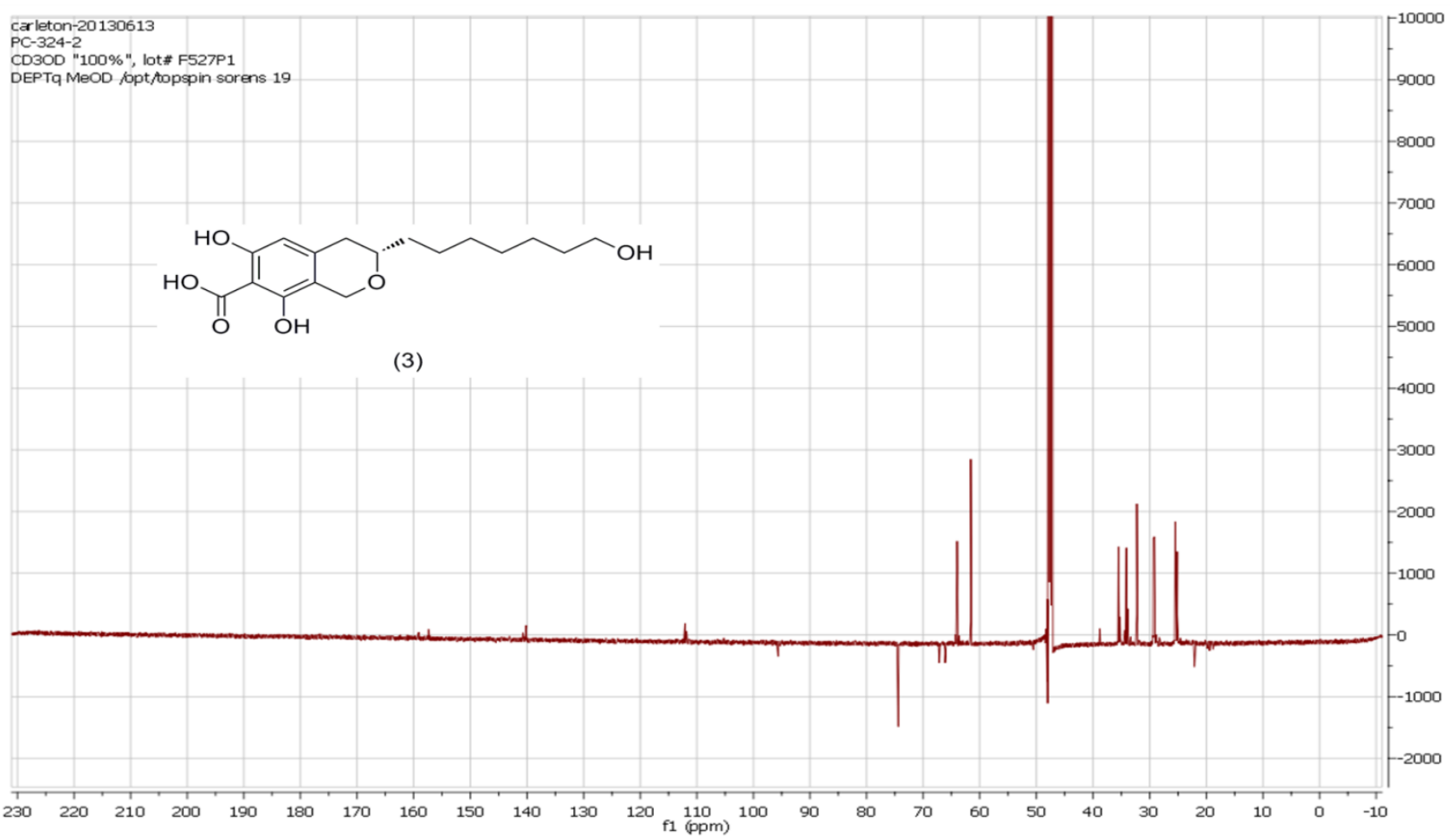


${ }^{1} \mathrm{H}-\mathrm{NMR}\left(\mathrm{CD}_{3} \mathrm{OD}, 700 \mathrm{MHz}\right)$ spectrum of compound $\mathbf{3 . 4}$

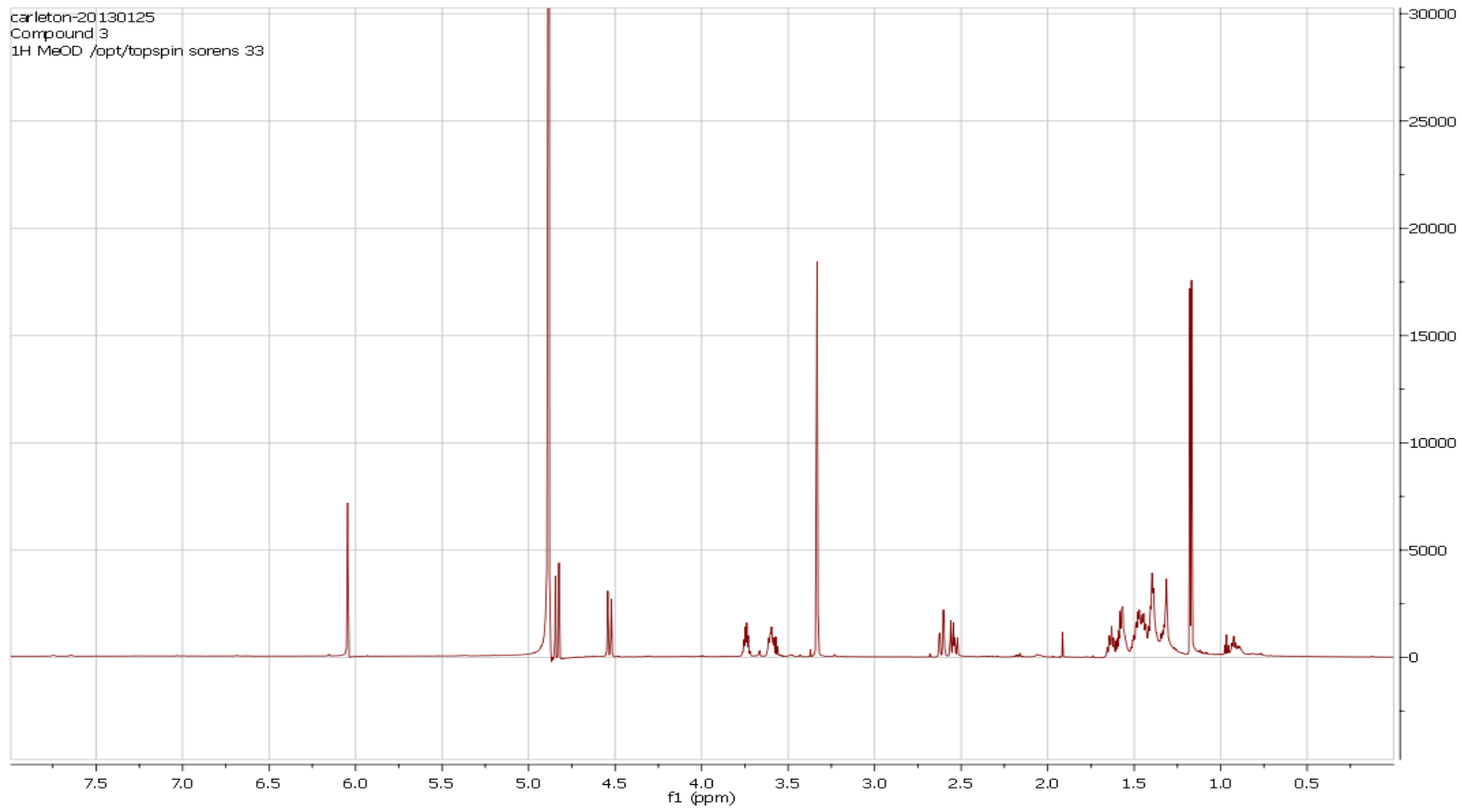

${ }^{13} \mathrm{C}-\mathrm{NMR}\left(\mathrm{CD}_{3} \mathrm{OD}, 175 \mathrm{MHz}\right)$ spectrum of compound $\mathbf{3 . 4}$

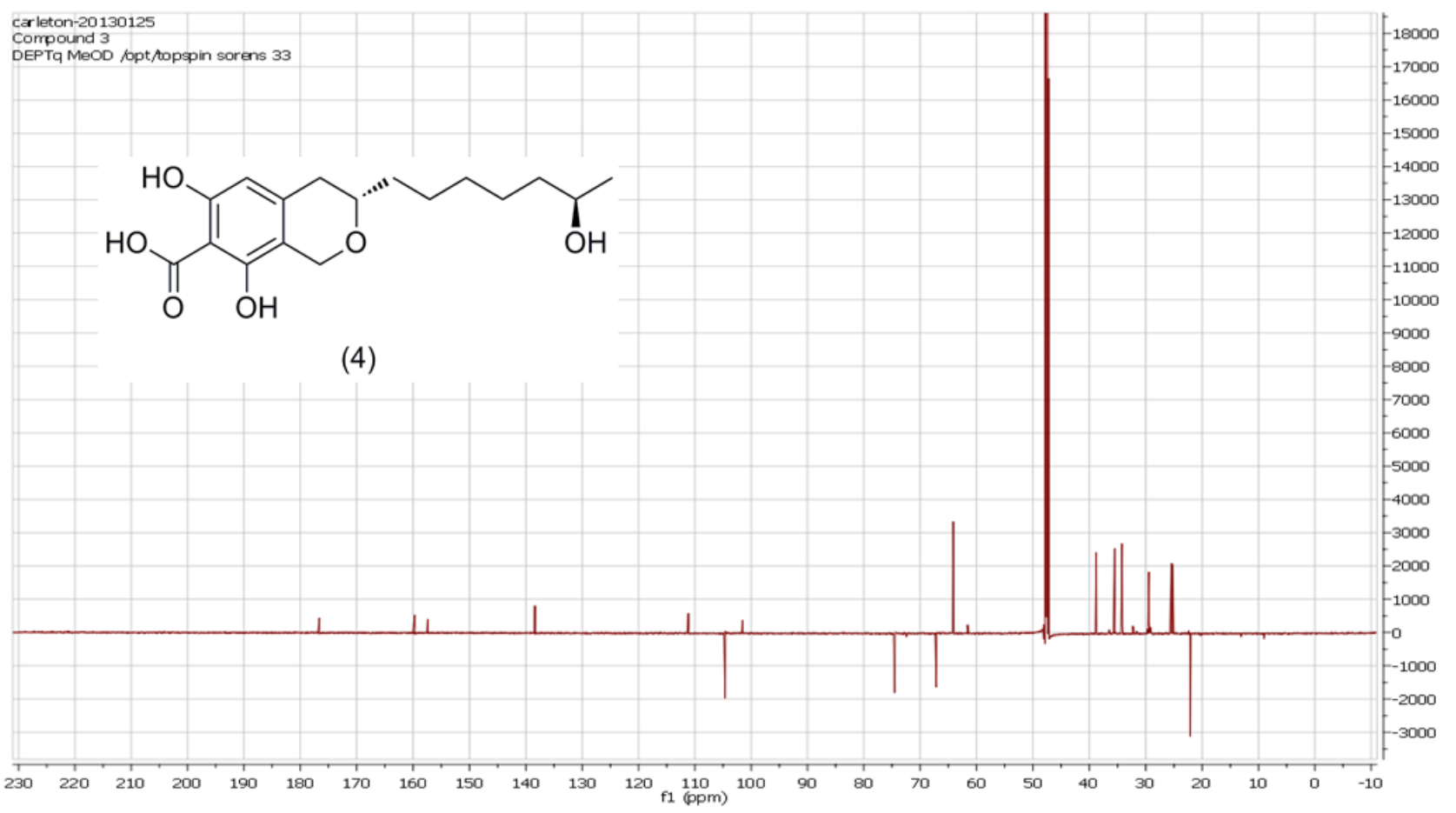


${ }^{1} \mathrm{H}-\mathrm{NMR}\left(\mathrm{CD}_{3} \mathrm{OD}, 700 \mathrm{MHz}\right)$ spectrum of compound 3.6

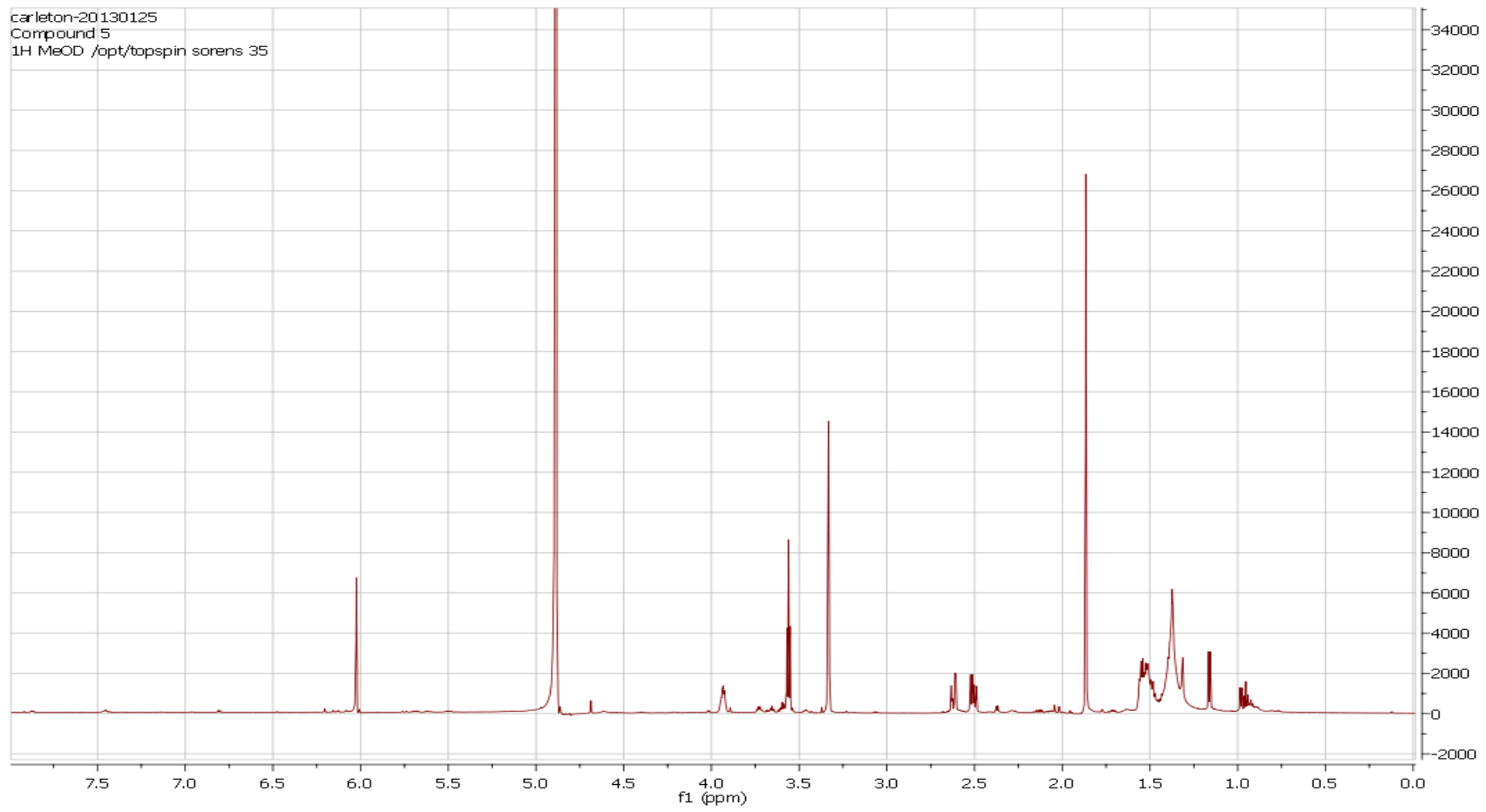

${ }^{13} \mathrm{C}-\mathrm{NMR}\left(\mathrm{CD}_{3} \mathrm{OD}, 175 \mathrm{MHz}\right)$ spectrum of compound $\mathbf{3 . 6}$

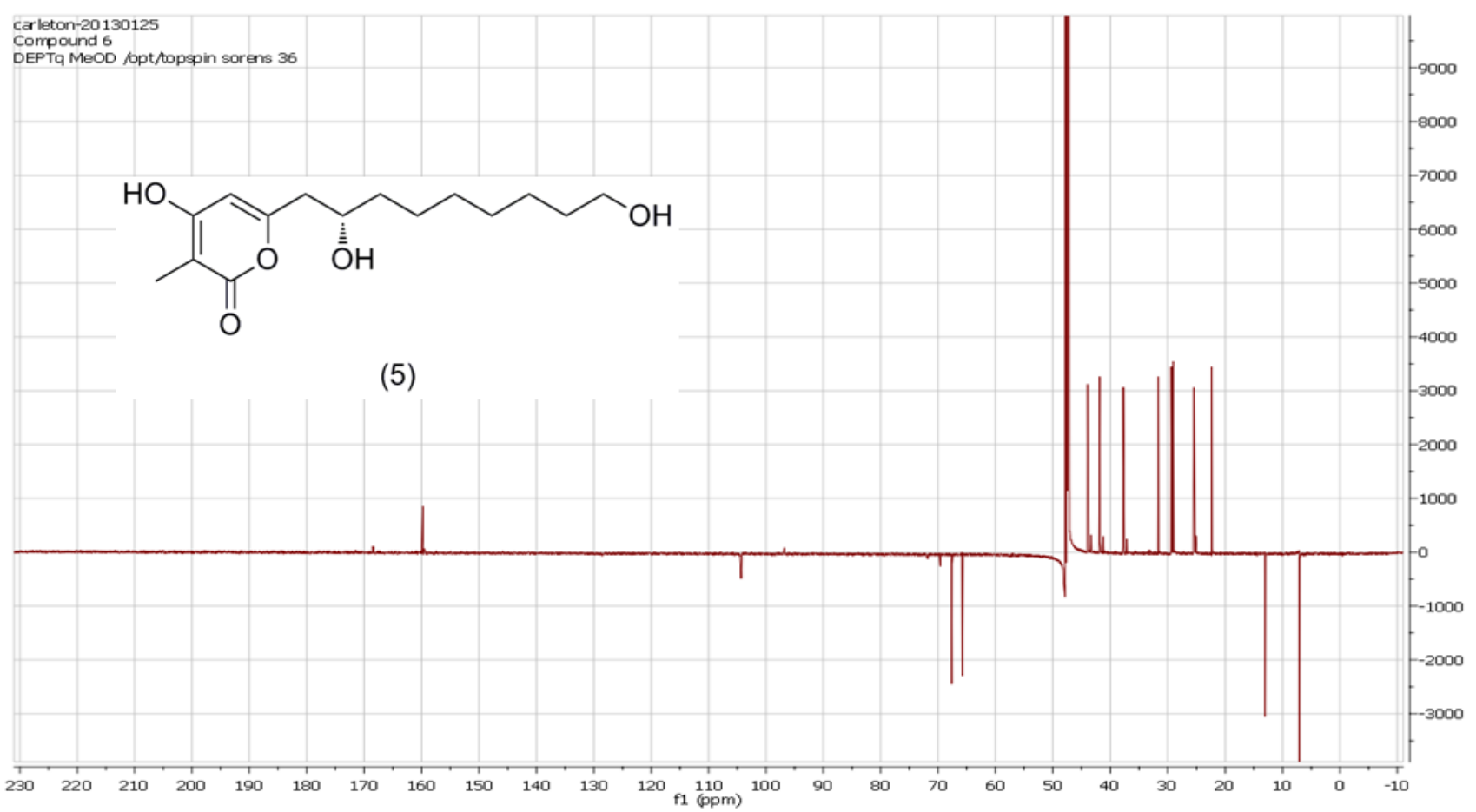




\section{${ }^{1} \mathrm{H}-\mathrm{NMR}\left(\mathrm{CD}_{3} \mathrm{OD}, 700 \mathrm{MHz}\right)$ spectrum of compound $\mathbf{3 . 7}$}

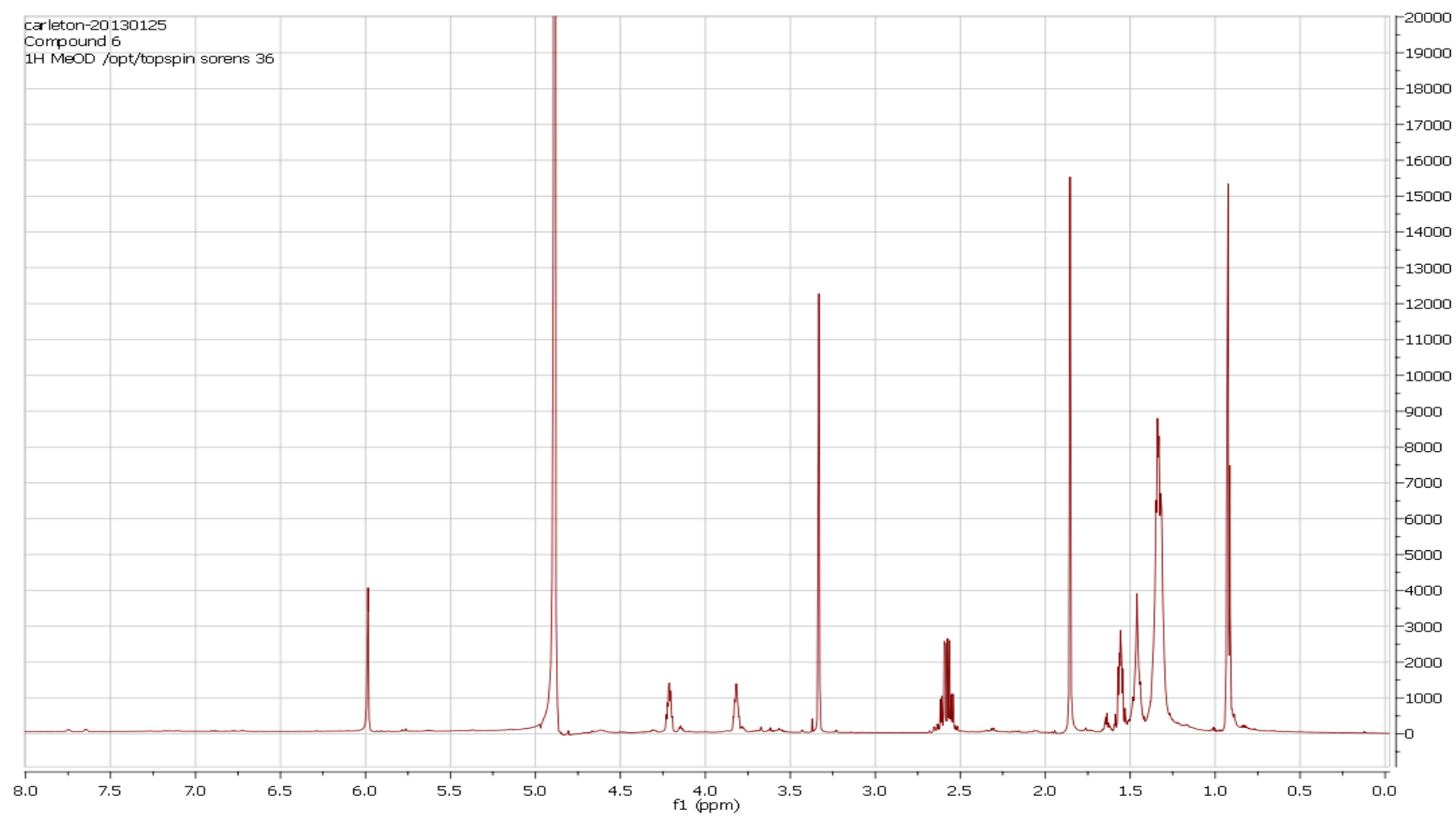

${ }^{13} \mathrm{C}-\mathrm{NMR}\left(\mathrm{CD}_{3} \mathrm{OD}, 175 \mathrm{MHz}\right)$ spectrum of compound 3.7

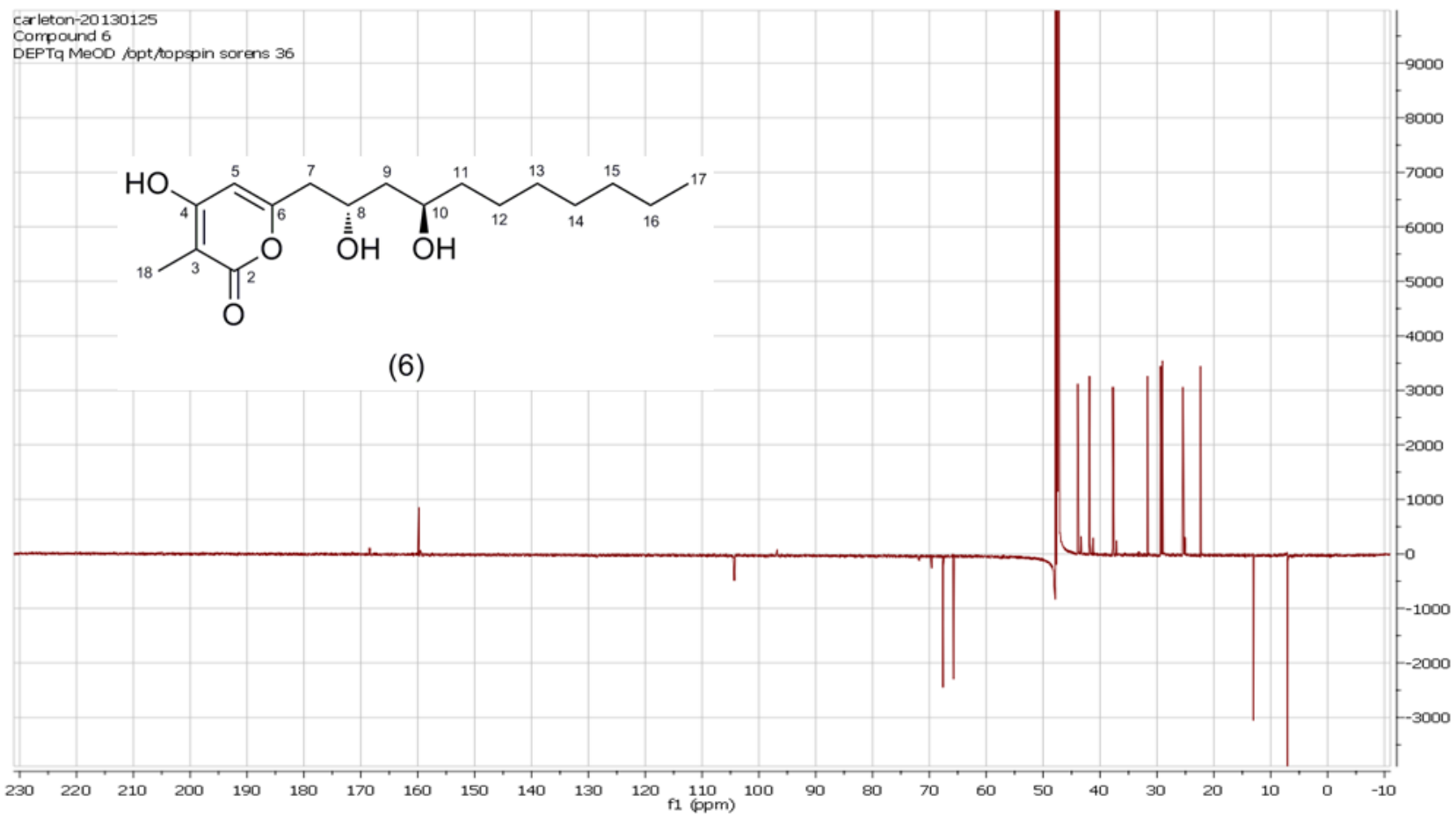


${ }^{1} \mathrm{H}-\mathrm{NMR}\left(\mathrm{CD}_{3} \mathrm{OD}, 700 \mathrm{MHz}\right)$ spectrum of compound $\mathbf{3 . 8}$

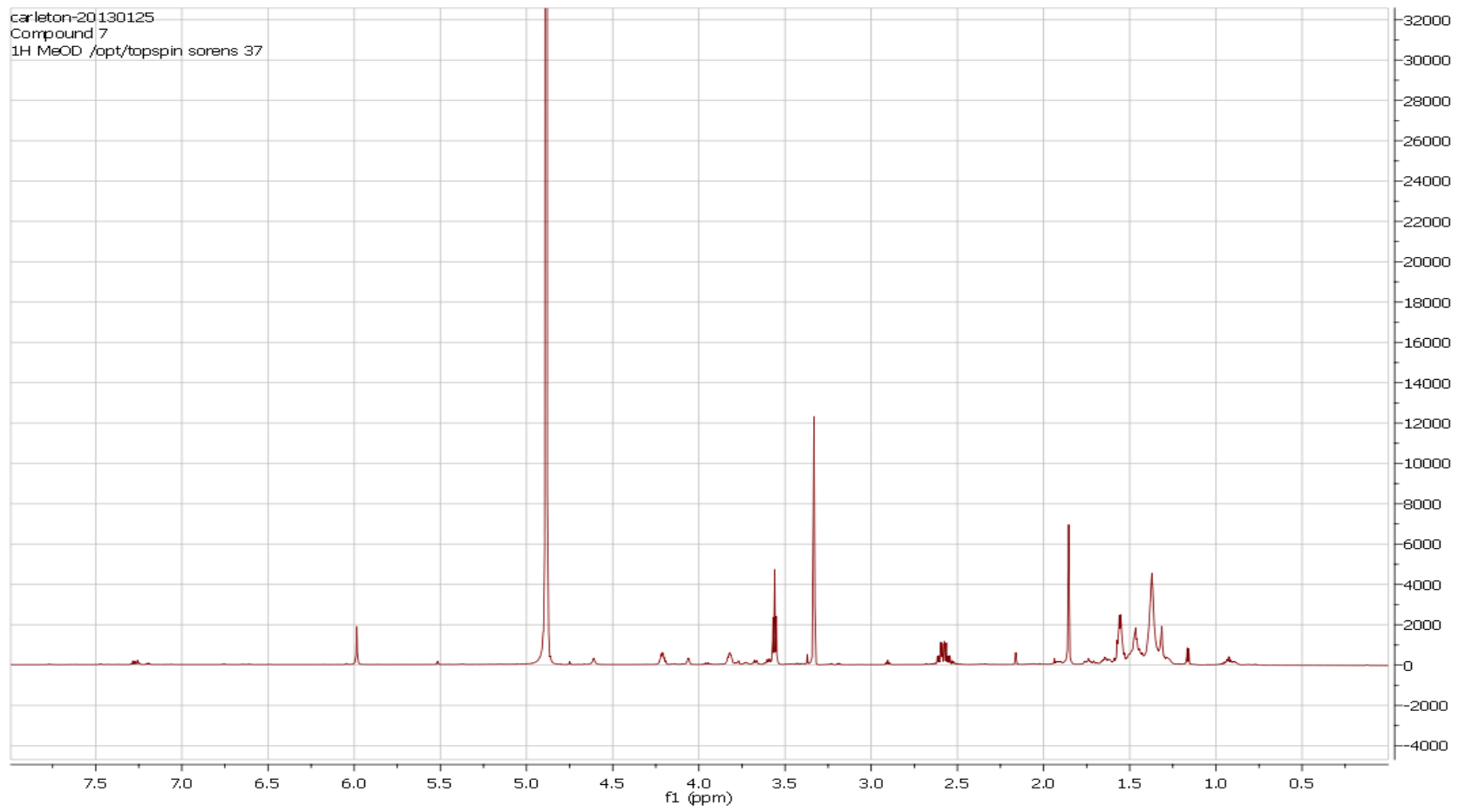

${ }^{13} \mathrm{C}-\mathrm{NMR}\left(\mathrm{CD}_{3} \mathrm{OD}, 175 \mathrm{MHz}\right)$ spectrum of compound $\mathbf{3 . 8}$

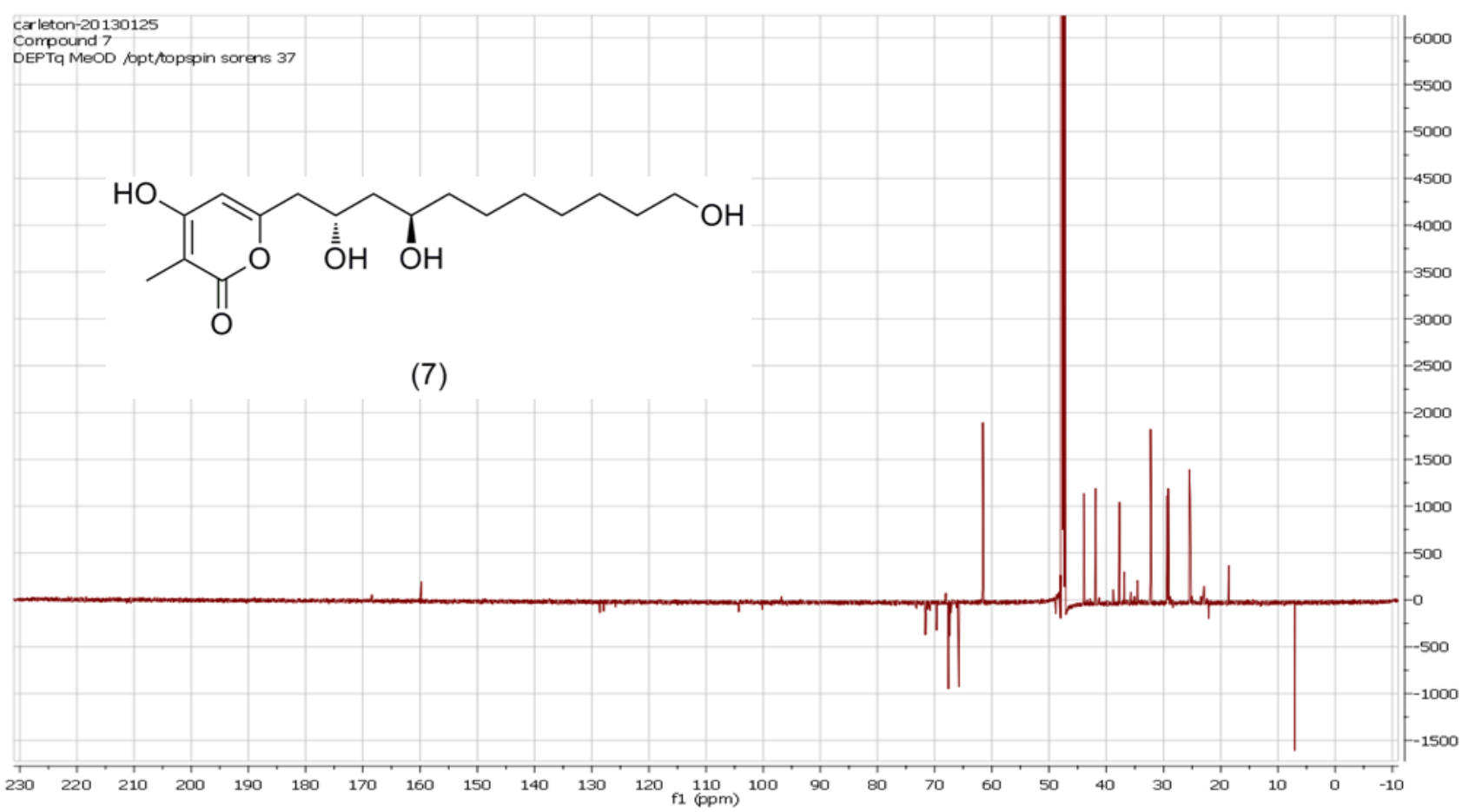


${ }^{1} \mathrm{H}-\mathrm{NMR}\left(\mathrm{CD}_{3} \mathrm{OD}, 400 \mathrm{MHz}\right)$ spectrum of $\mathbf{3 . 5}$

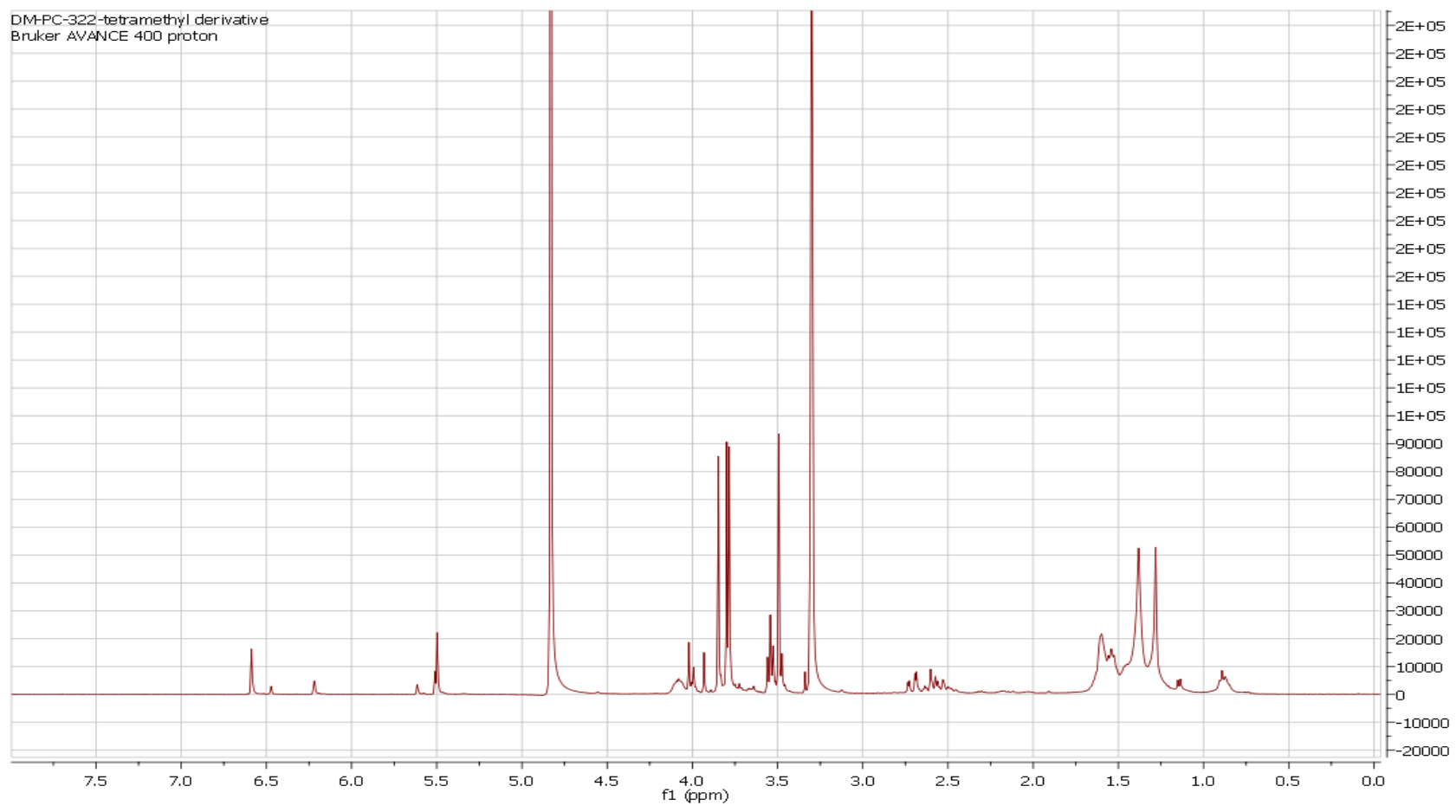

${ }^{13} \mathrm{C}-\mathrm{NMR}\left(\mathrm{CD}_{3} \mathrm{OD}, 100 \mathrm{MHz}\right)$ spectrum of $\mathbf{3 . 5}$

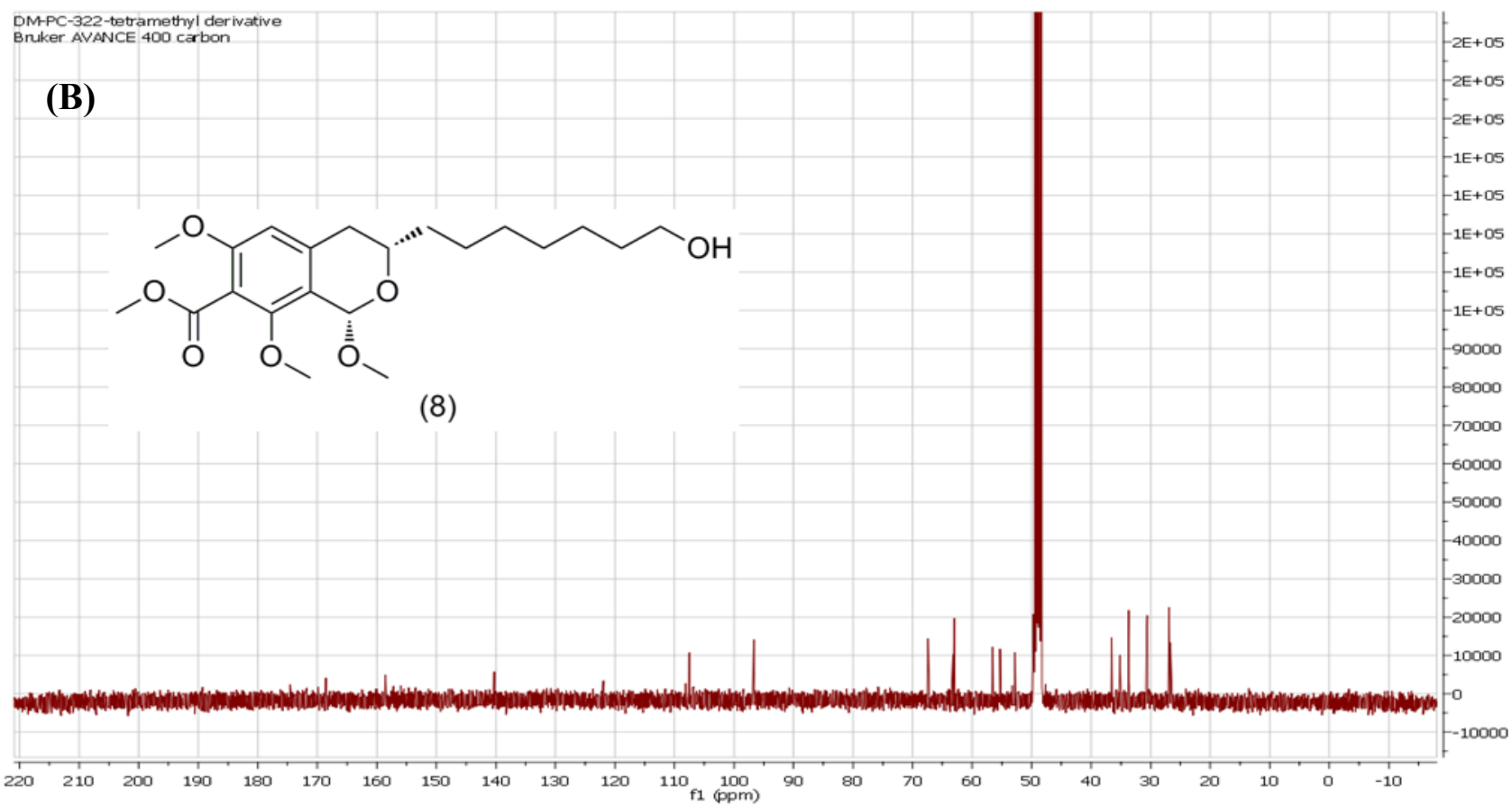


${ }^{1} \mathrm{H}-\mathrm{NMR}\left(\mathrm{CD}_{3} \mathrm{OD}, 700 \mathrm{MHz}\right)$ spectrum of 1:1 mixture of compounds $\mathbf{3 . 1}$ and $\mathbf{3 . 2}$

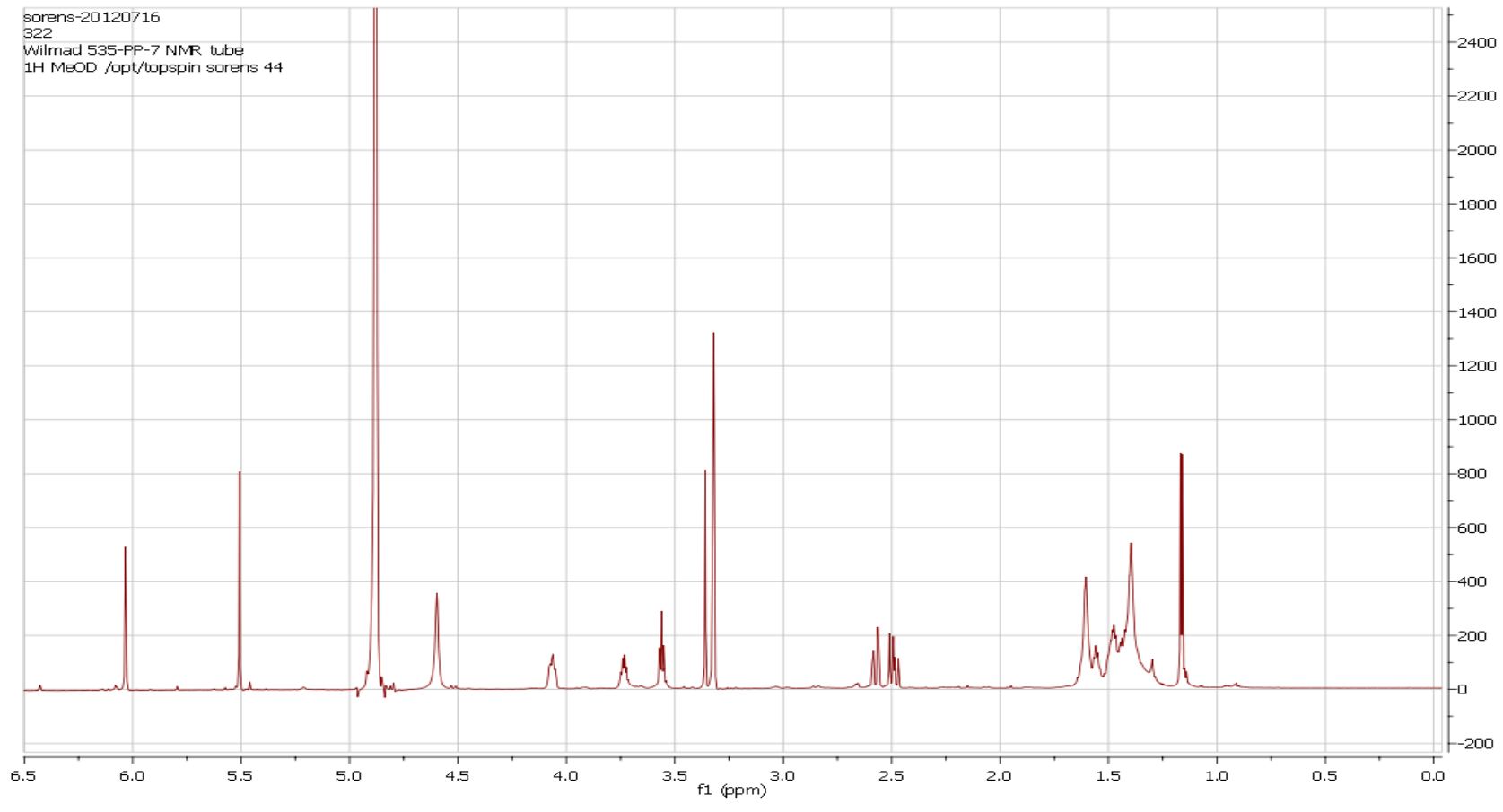

${ }^{13} \mathrm{C}-\mathrm{NMR}\left(\mathrm{CD}_{3} \mathrm{OD}, 175 \mathrm{MHz}\right)$ spectrum of a 1:1 mixture of compounds $\mathbf{3 . 1}$ and $\mathbf{3 . 2}$

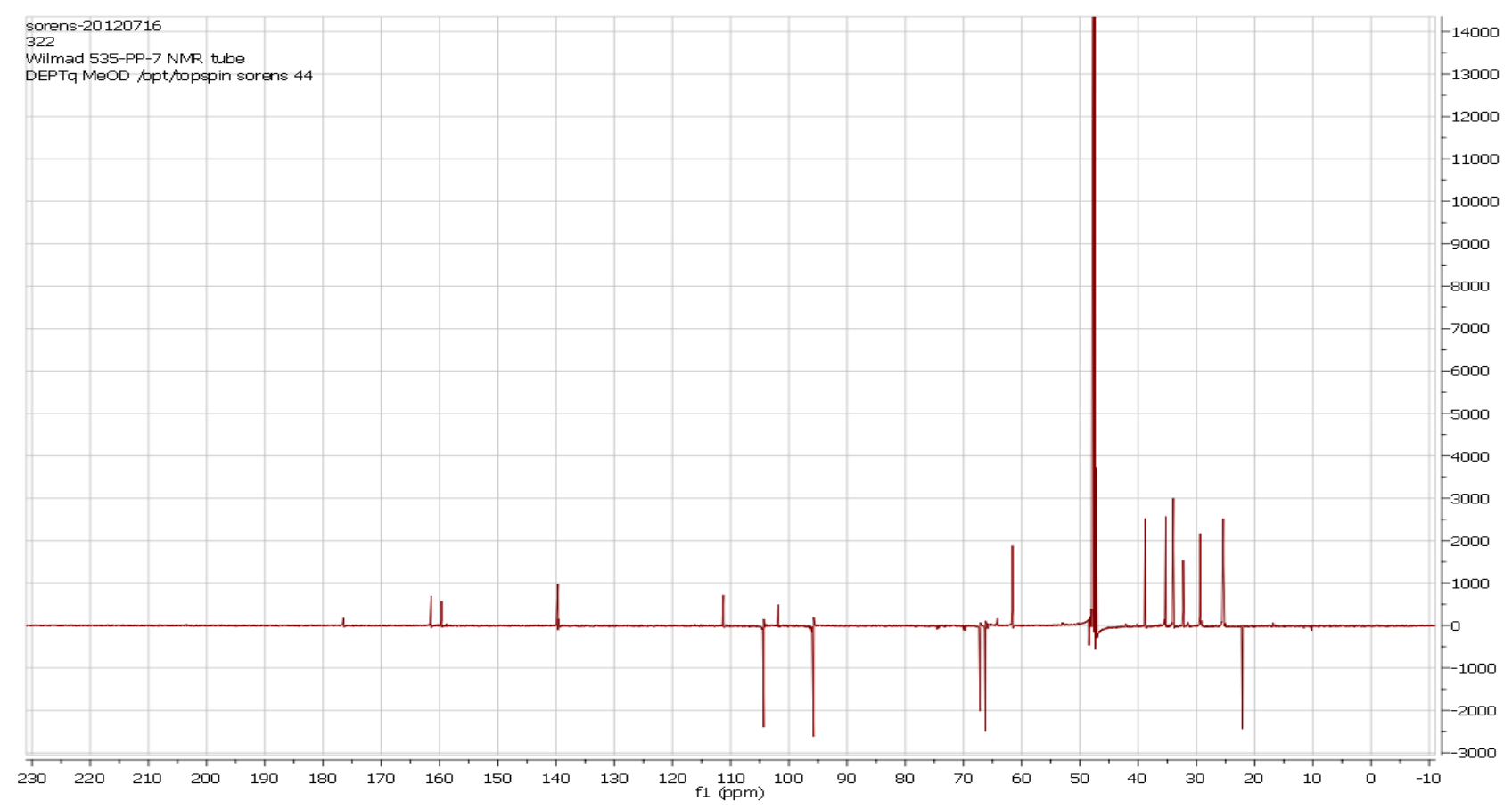




\section{${ }^{1} \mathrm{H}-\mathrm{NMR}\left(\mathrm{CD}_{3} \mathrm{OD}, 400 \mathrm{MHz}\right)$ spectrum of $\mathbf{3 . 9}$}

DM-P. cory 264 comp (Phomenone)/1 1D PROTON EXPERIMENT

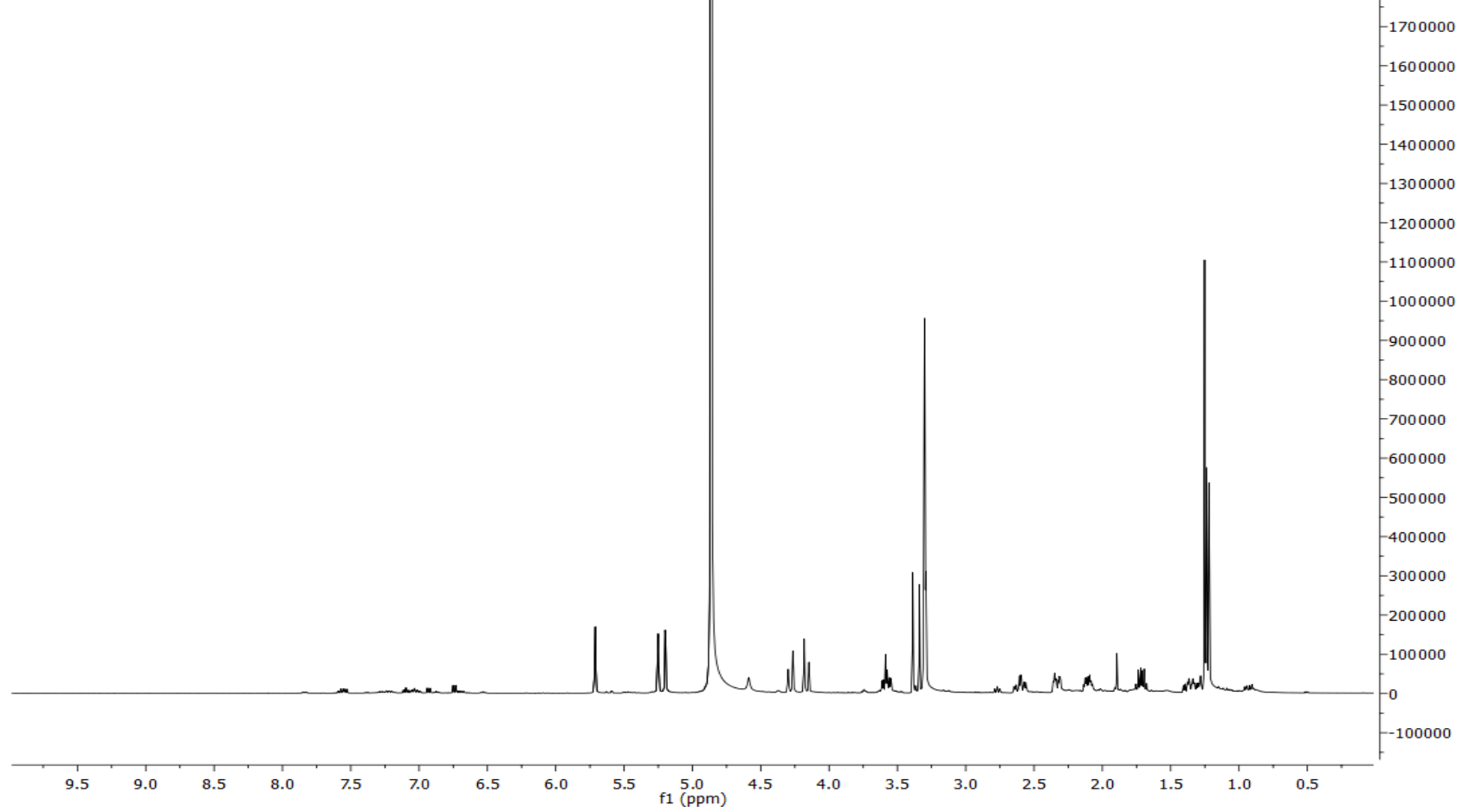

${ }^{13} \mathrm{C}-\mathrm{NMR}\left(\mathrm{CD}_{3} \mathrm{OD}, 100 \mathrm{MHz}\right)$ spectrum of $\mathbf{3 . 9}$ DM-P. cory 264 cmp (Phomenone)/2
1D C13 Experiment

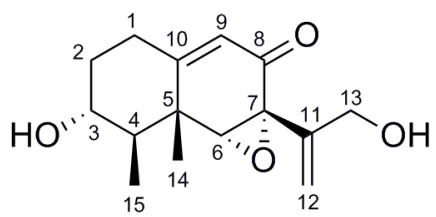

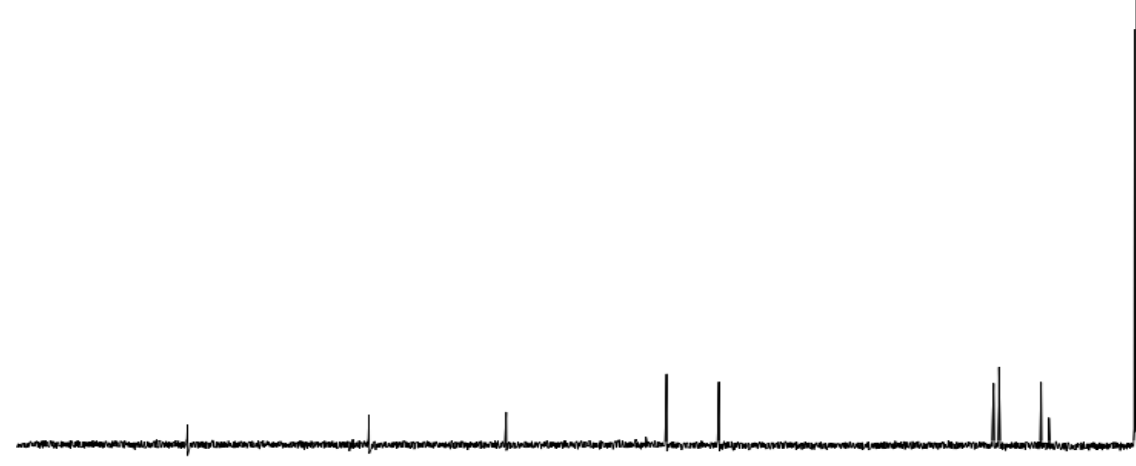

$-300000$

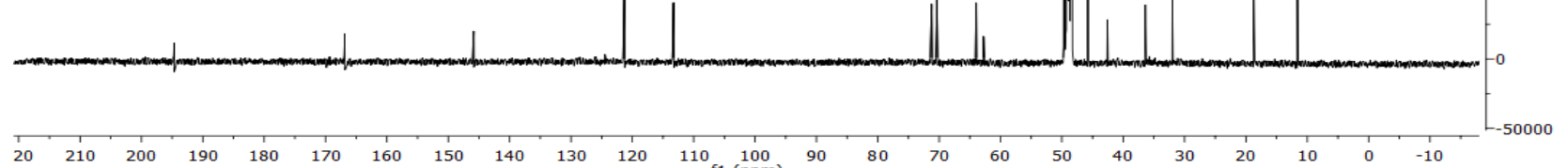


${ }^{1} \mathrm{H}-\mathrm{NMR}\left(\mathrm{CD}_{3} \mathrm{OD}, 400 \mathrm{MHz}\right)$ spectrum of $\mathbf{3 . 1 0}$

DM- P.cory 486/Proton

1D PROTON EXPERIMENT

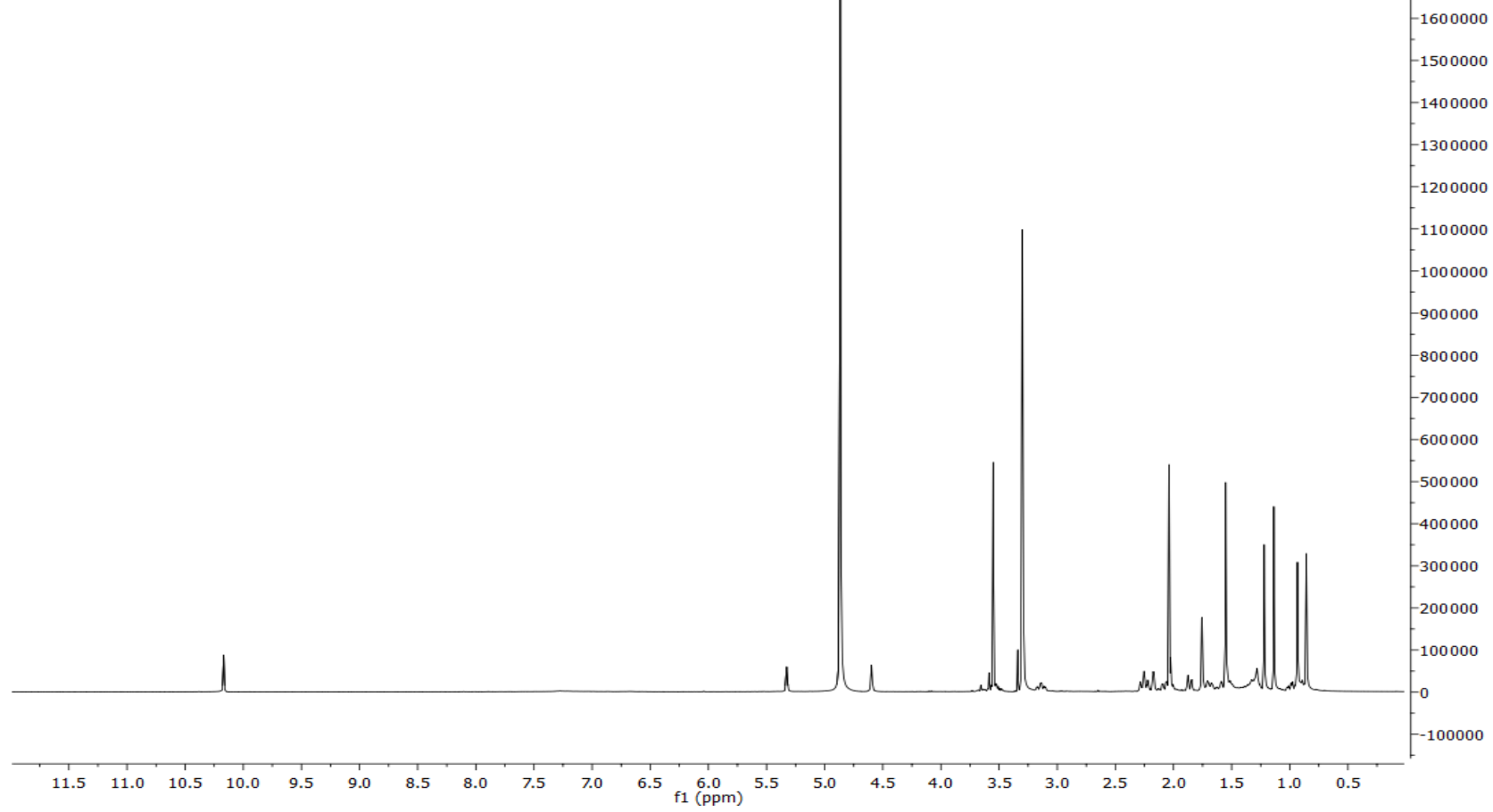

${ }^{13} \mathrm{C}-\mathrm{NMR}\left(\mathrm{CD}_{3} \mathrm{OD}, 100 \mathrm{MHz}\right)$ spectrum of $\mathbf{3 . 9}$

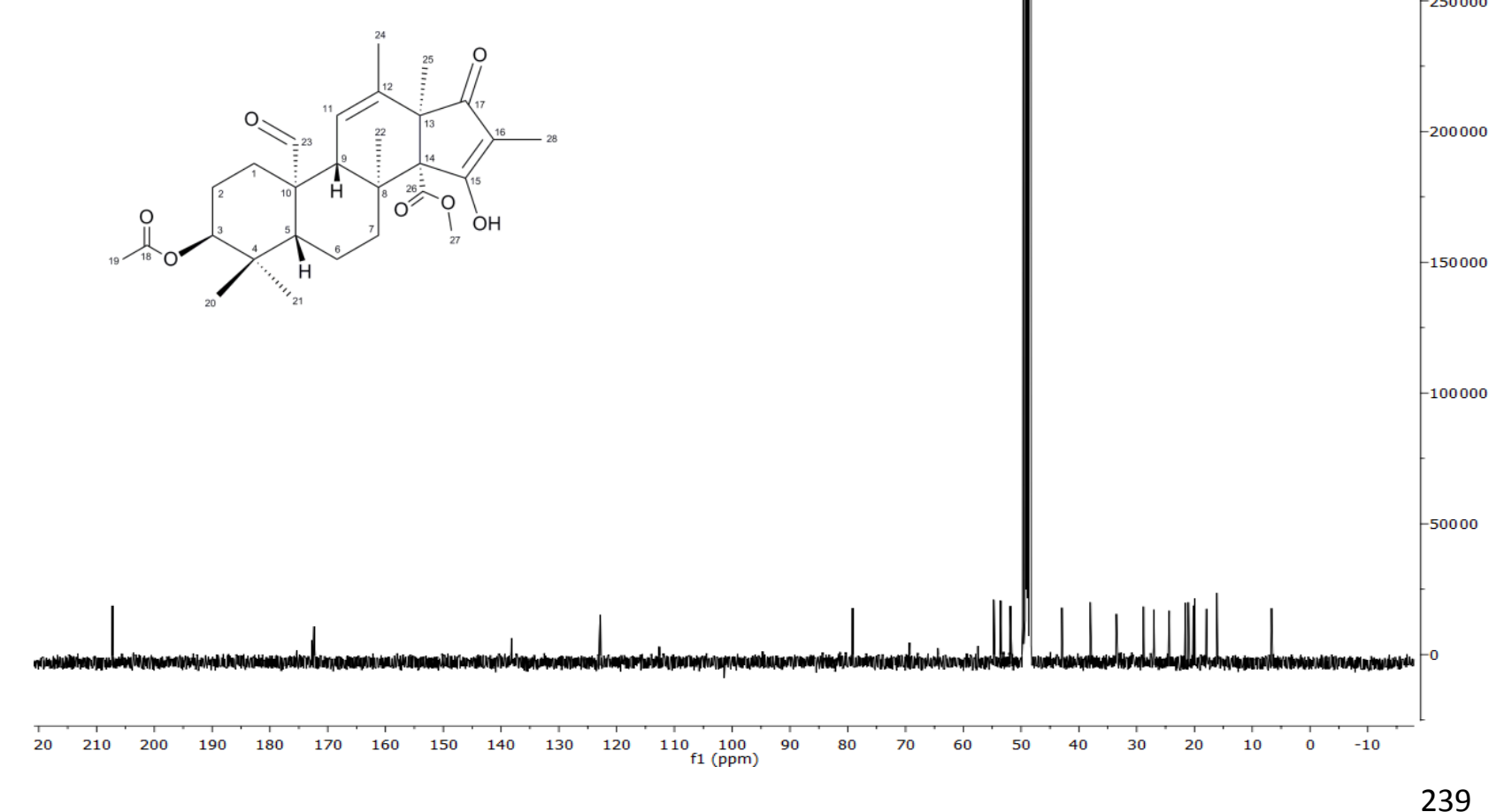




\section{${ }^{1} \mathrm{H}-\mathrm{NMR}\left(\mathrm{CD}_{3} \mathrm{OD}, 400 \mathrm{MHz}\right)$ spectrum of $\mathbf{3 . 1 1}$}

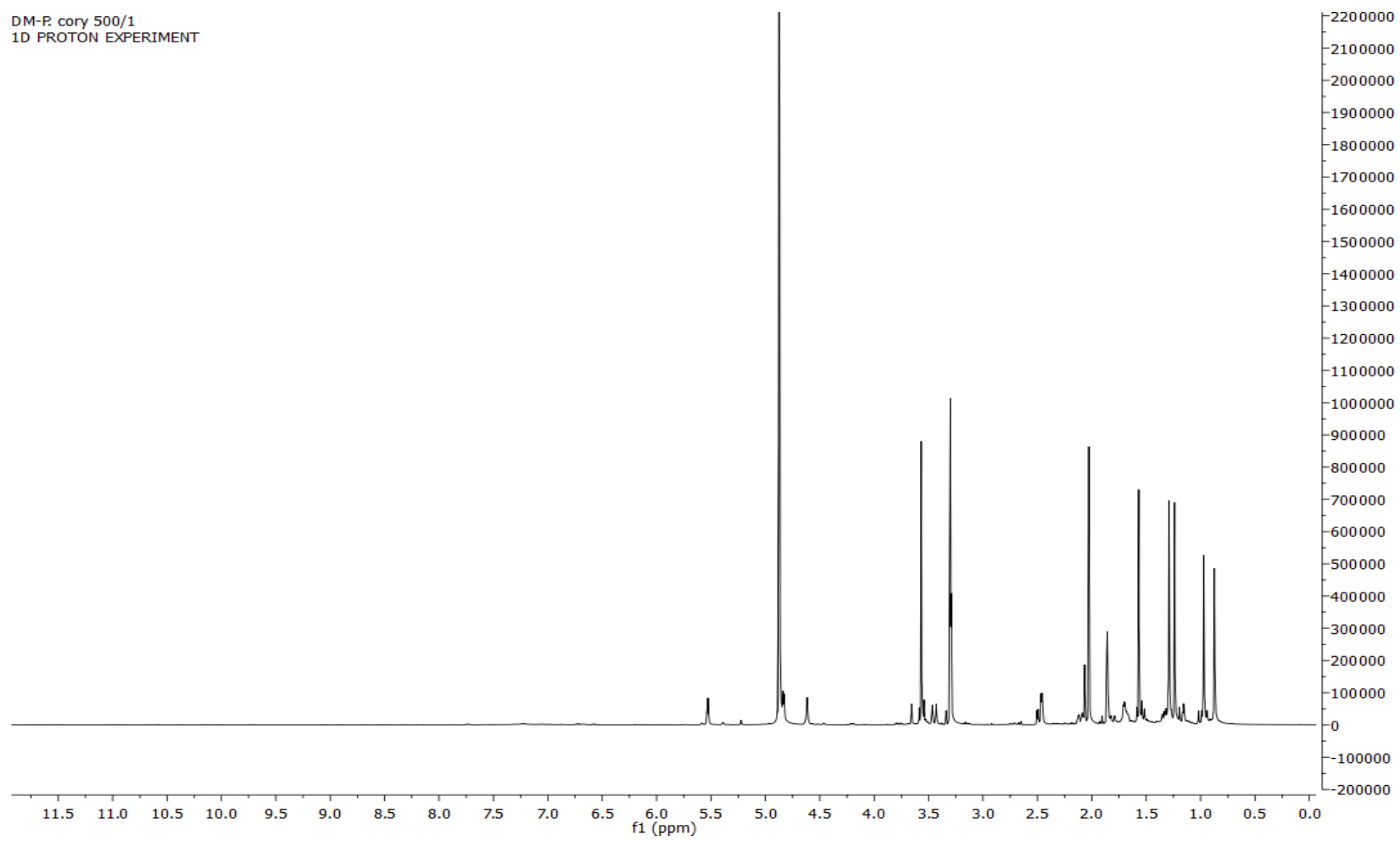

${ }^{13} \mathrm{C}-\mathrm{NMR}\left(\mathrm{CD}_{3} \mathrm{OD}, 100 \mathrm{MHz}\right)$ spectrum of $\mathbf{3 . 1 1}$

DM-P. cory 500/2

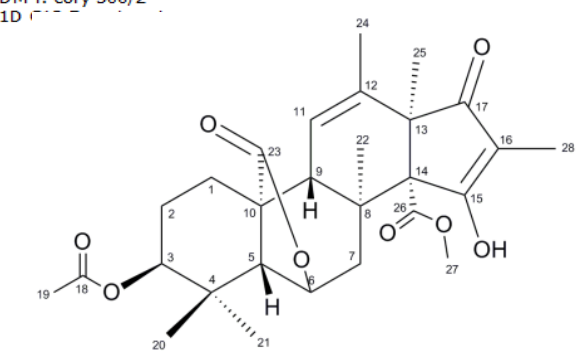




\section{${ }^{1} \mathrm{H}-\mathrm{NMR}\left(\mathrm{CD}_{3} \mathrm{OD}, 400 \mathrm{MHz}\right)$ spectrum of $\mathbf{3 . 1 2}$}

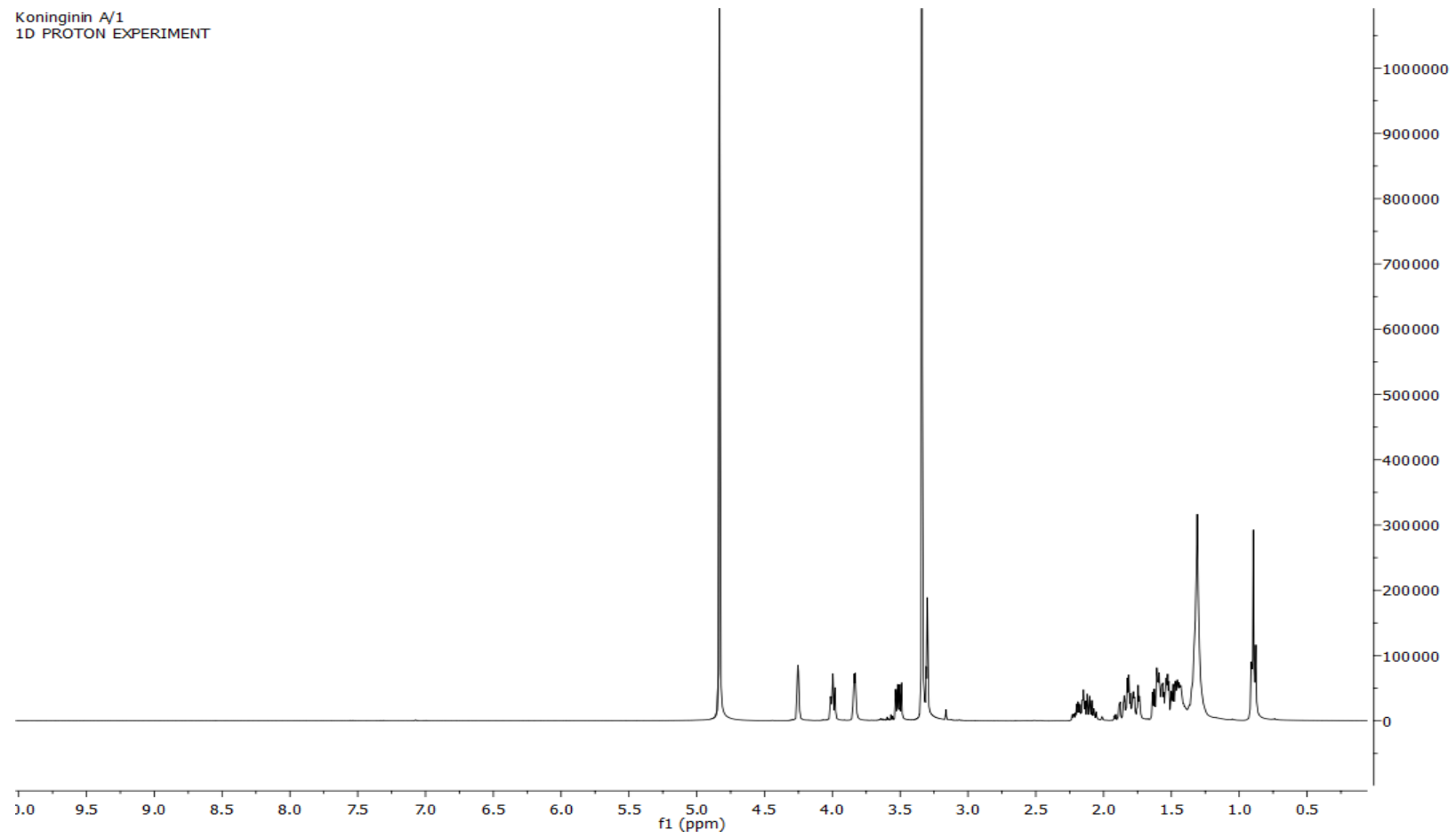

${ }^{13} \mathrm{C}-\mathrm{NMR}\left(\mathrm{CD}_{3} \mathrm{OD}, 100 \mathrm{MHz}\right)$ spectrum of $\mathbf{3 . 1 2}$

Koninginin $\mathrm{A} / 2$
$1 \mathrm{D}$ C13 Experiment
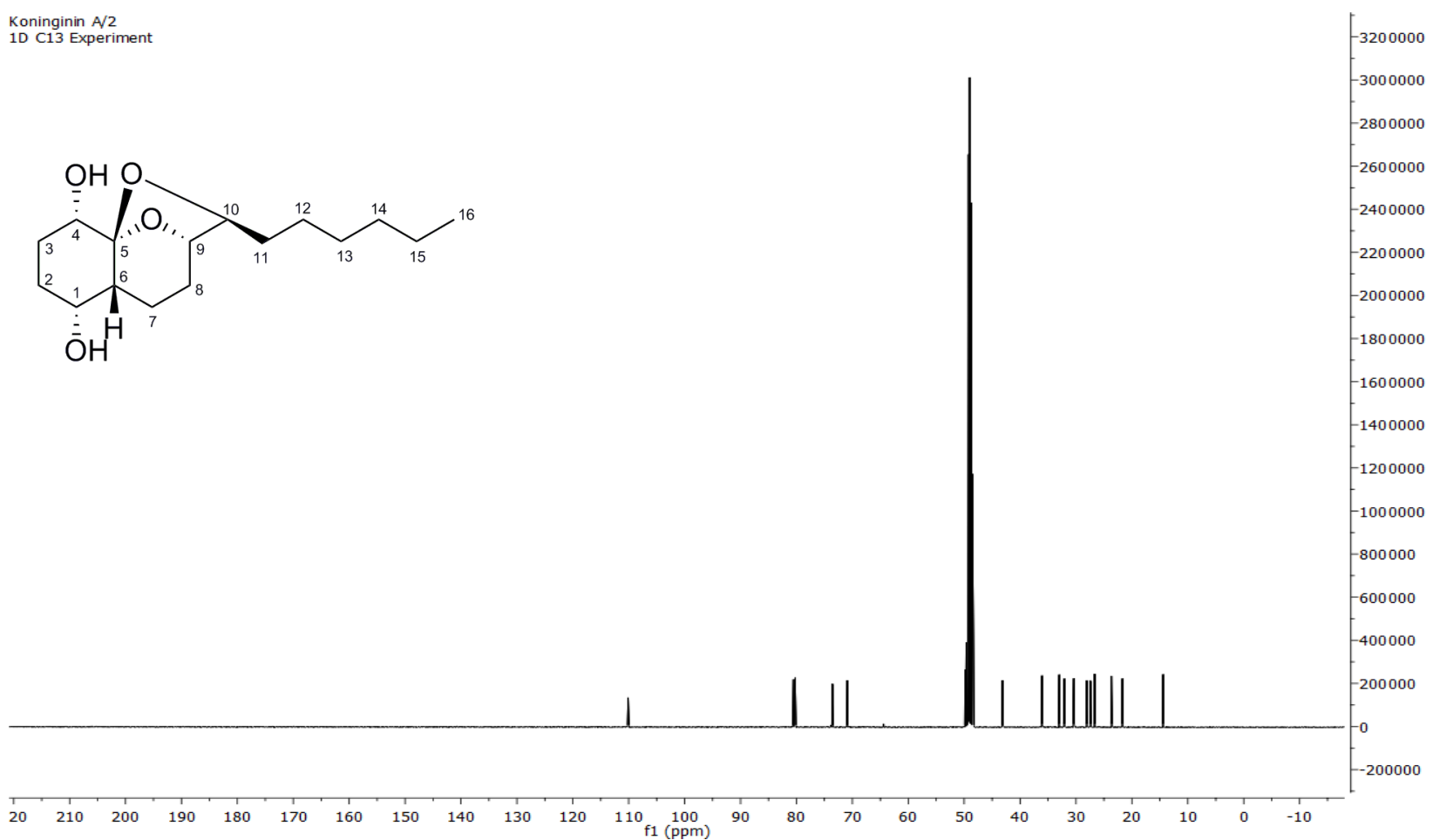


\section{${ }^{1} \mathrm{H}-\mathrm{NMR}\left(\mathrm{CD}_{3} \mathrm{OD}, 400 \mathrm{MHz}\right)$ spectrum of $\mathbf{3 . 1 3}$}

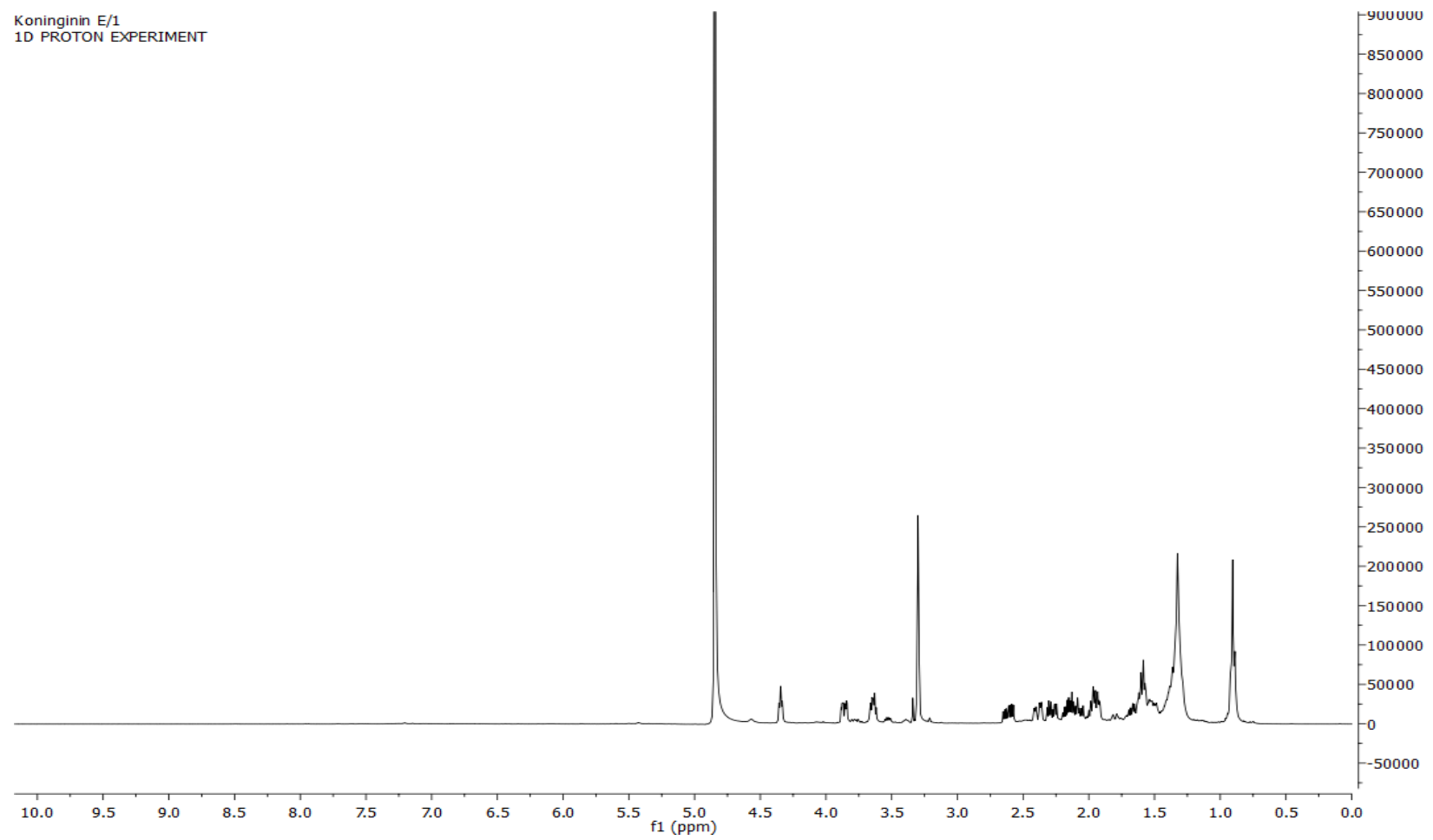

${ }^{13} \mathrm{C}-\mathrm{NMR}\left(\mathrm{CD}_{3} \mathrm{OD}, 100 \mathrm{MHz}\right)$ spectrum of $\mathbf{3 . 1 3}$

Koninginin $E / 2$

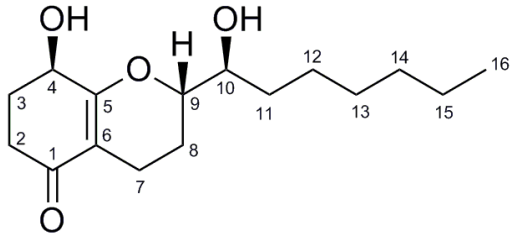

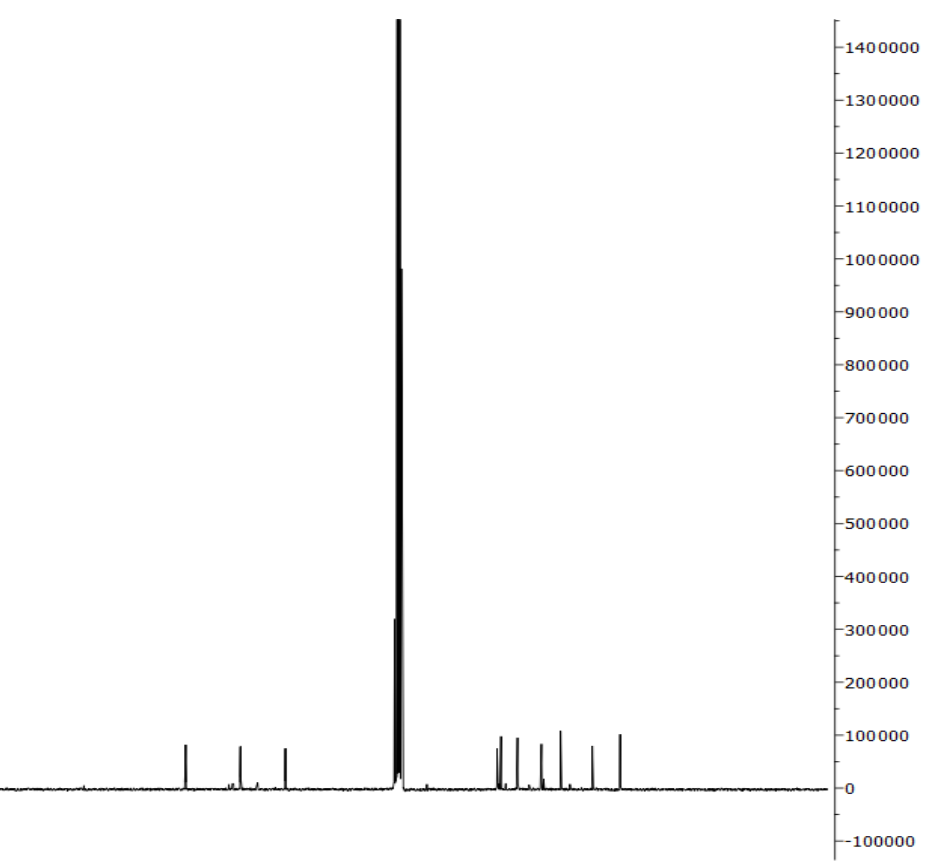

$\begin{array}{llllllllllllllllllllllllll}20 & 210 & 200 & 190 & 180 & 170 & 160 & 150 & 140 & 130 & 120 & 110 & 100 & 90 & 80 & 70 & 60 & 50 & 40 & 30 & 20 & 10 & 0 & -10 & \end{array}$ 


\section{${ }^{1} \mathrm{H}-\mathrm{NMR}\left(\mathrm{CD}_{3} \mathrm{OD}, 400 \mathrm{MHz}\right)$ spectrum of $\mathbf{3 . 1 4}$}

Koninginin G/1

1D PROTON EXPERIMENT

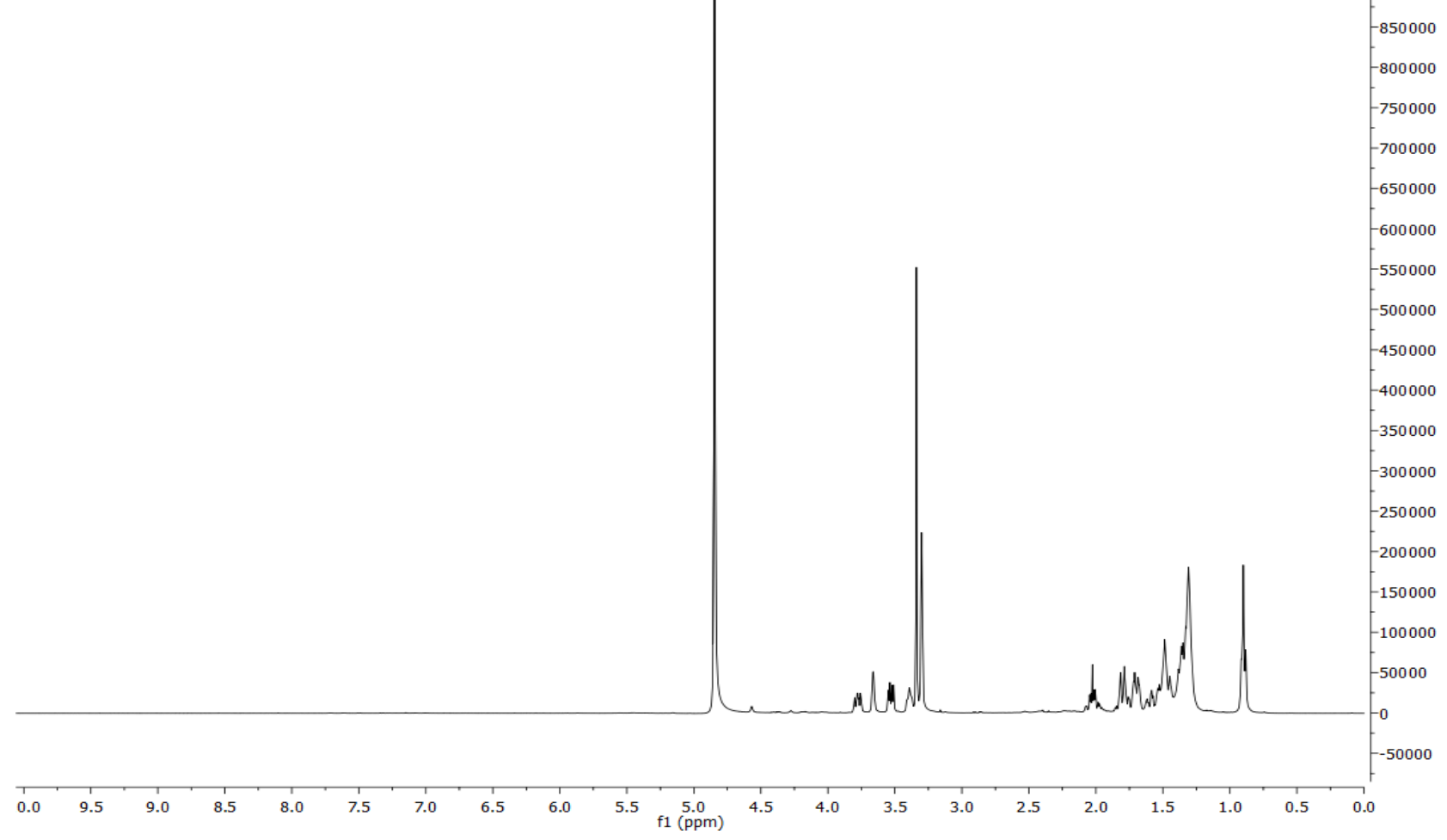

${ }^{13} \mathrm{C}-\mathrm{NMR}\left(\mathrm{CD}_{3} \mathrm{OD}, 100 \mathrm{MHz}\right)$ spectrum of $\mathbf{3 . 1 4}$

Koninginin $G / 2$

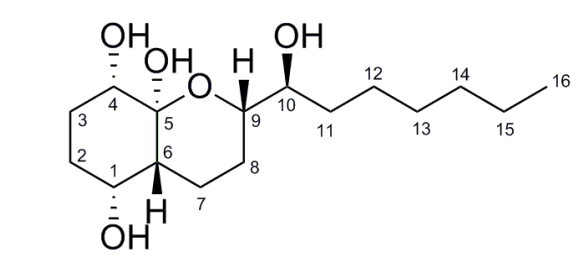


${ }^{1} \mathrm{H}-\mathrm{NMR}\left(\mathrm{CD}_{3} \mathrm{OD}, 400 \mathrm{MHz}\right)$ spectrum of $\mathbf{4 . 1}$

DM-WS-252-œmplete/1

Bruker AVANCE 400 proton

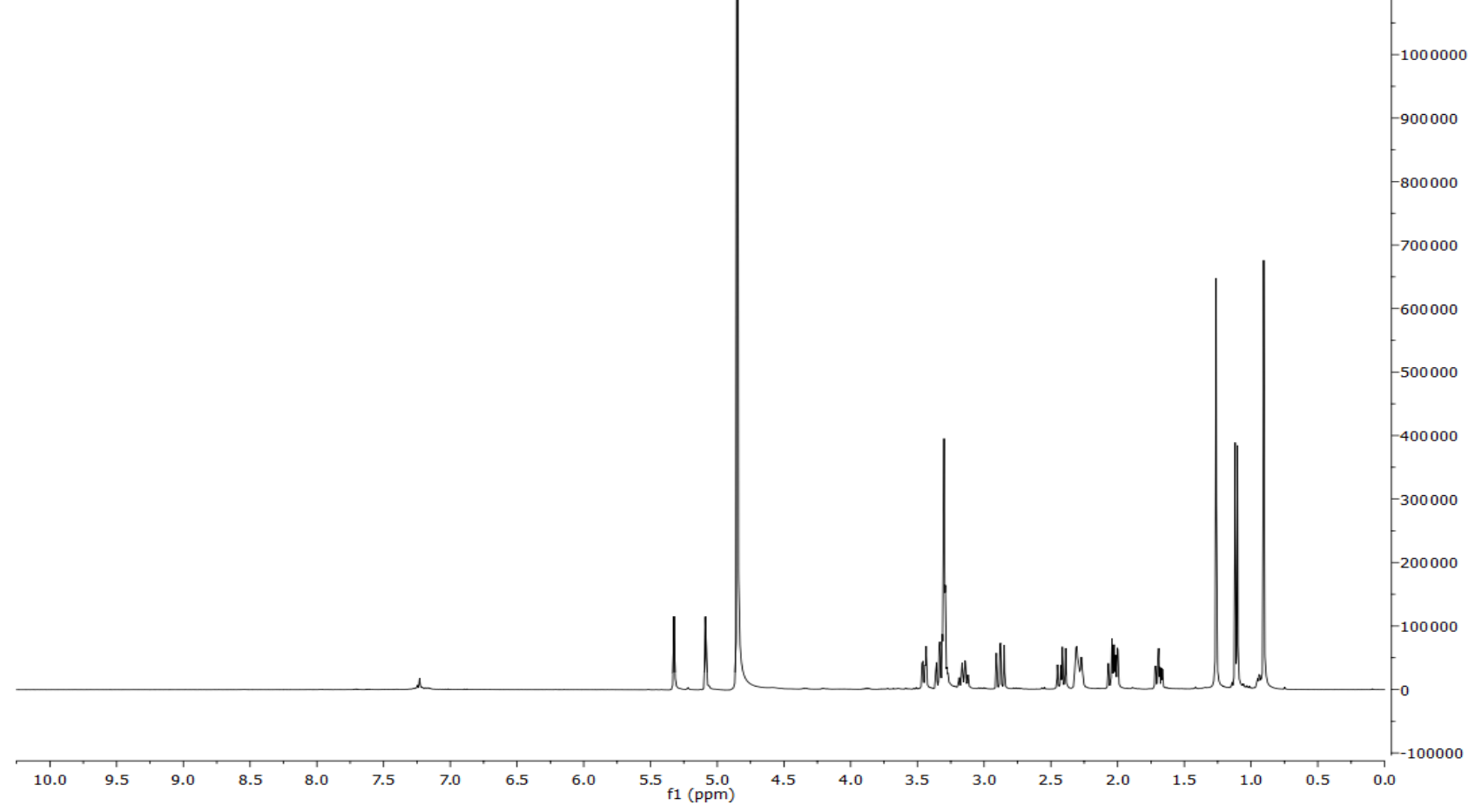

${ }^{13} \mathrm{C}-\mathrm{NMR}\left(\mathrm{CD}_{3} \mathrm{OD}, 100 \mathrm{MHz}\right)$ spectrum of 4.1

DM-WS-252-complete/2
Bruker AVANCE 400 carbon

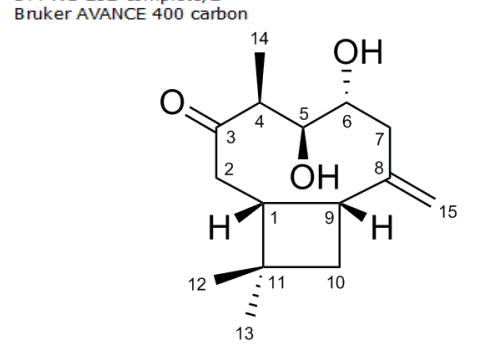

) spectrum of 4.1 


\section{${ }^{1} \mathrm{H}-\mathrm{NMR}\left(\mathrm{CD}_{3} \mathrm{OD}, 400 \mathrm{MHz}\right)$ spectrum of $\mathbf{4 . 2}$}

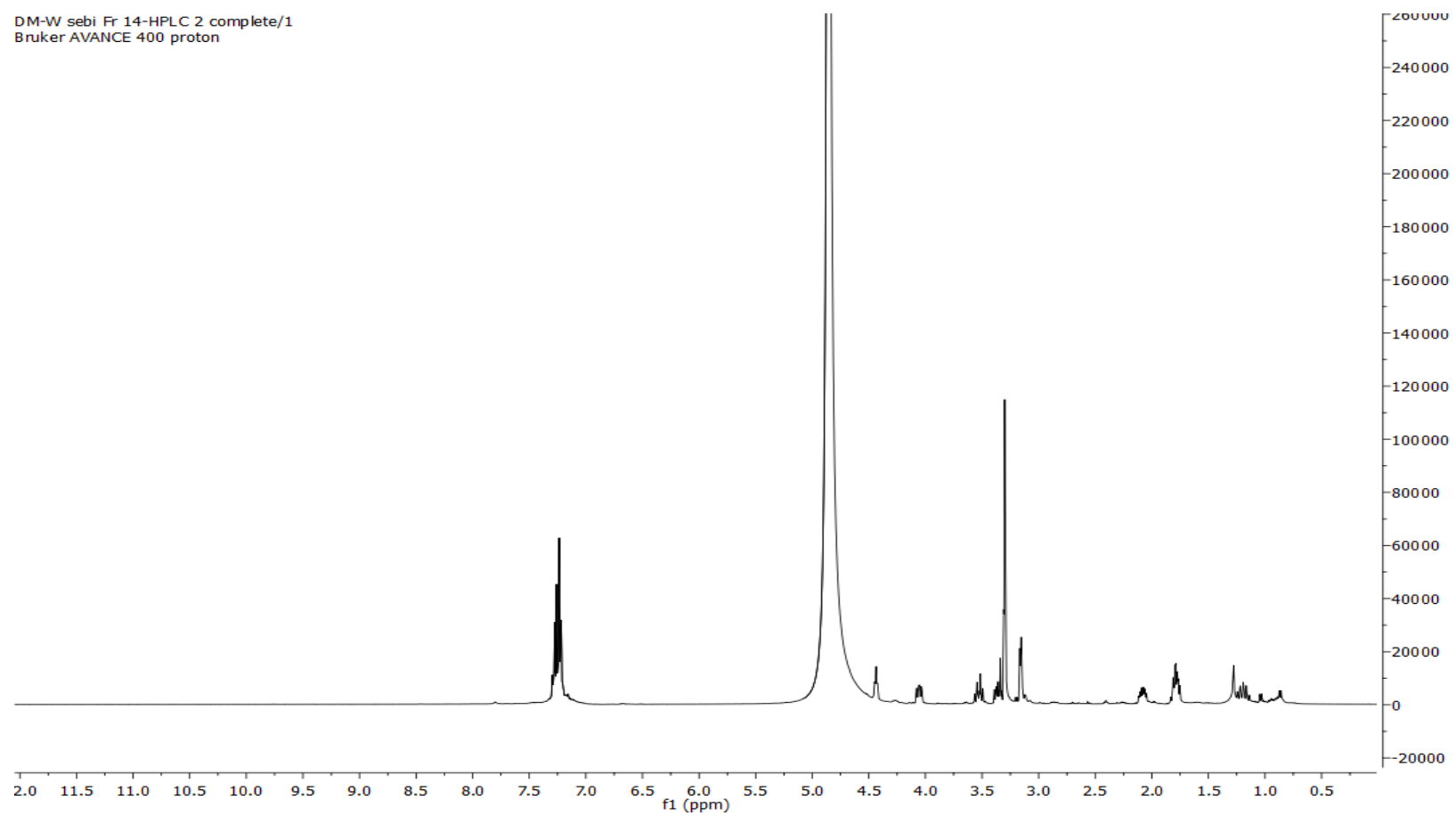

${ }^{13} \mathrm{C}-\mathrm{NMR}\left(\mathrm{CD}_{3} \mathrm{OD}, 100 \mathrm{MHz}\right)$ spectrum of $\mathbf{4 . 2}$

DM-W sebi Fr 14-HPLC 2 complete/2 Bruker AVANCE 400 carbon
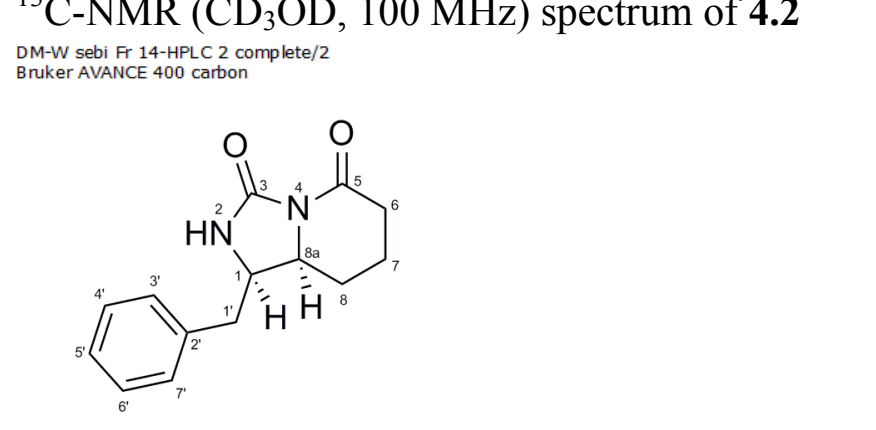


\section{${ }^{1} \mathrm{H}-\mathrm{NMR}\left(\mathrm{CD}_{3} \mathrm{OD}, 400 \mathrm{MHz}\right)$ spectrum of $\mathbf{4 . 3}$}

DM-tryptophol data

1D PROTON EXPERIMENT

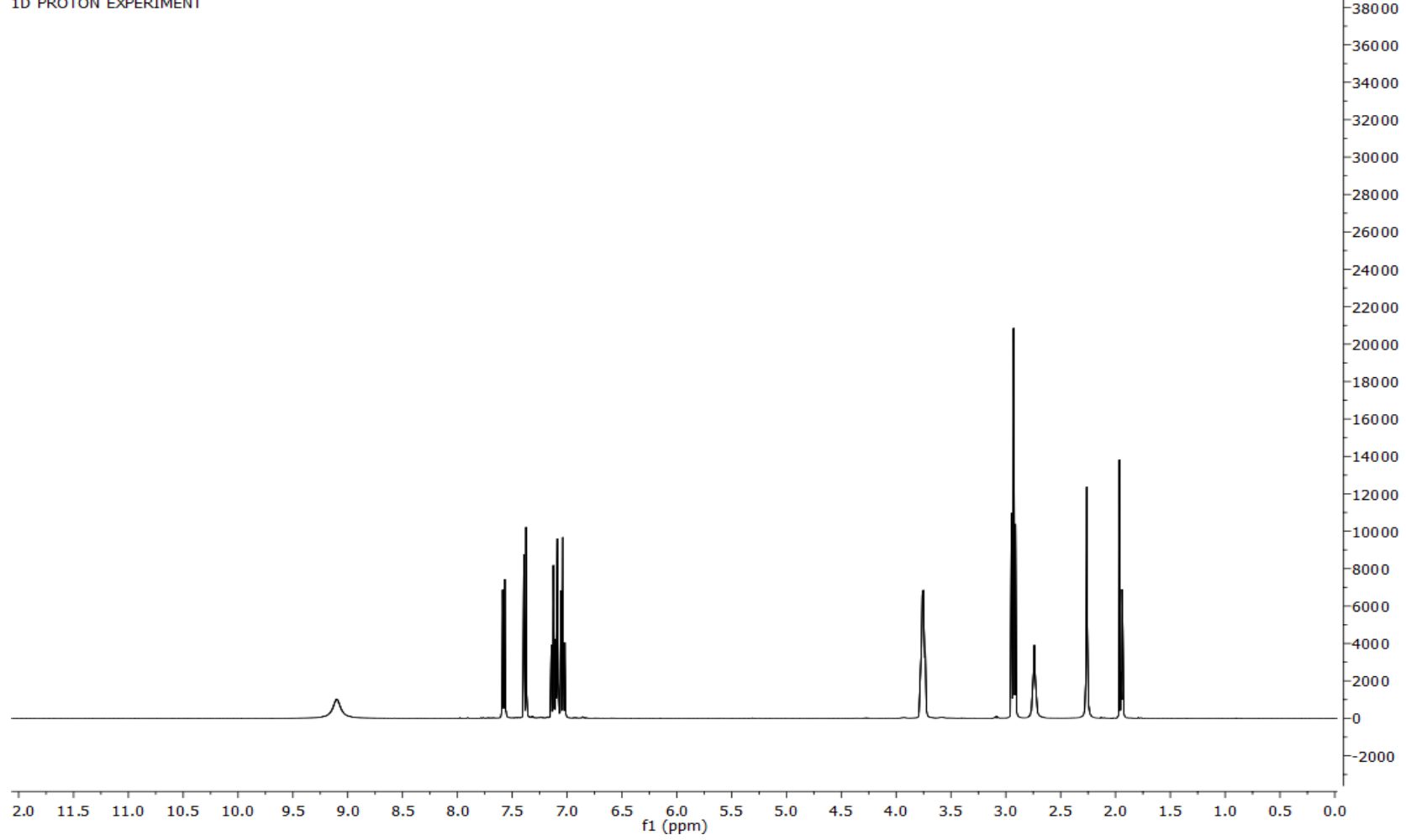

${ }^{13} \mathrm{C}-\mathrm{NMR}\left(\mathrm{CD}_{3} \mathrm{OD}, 100 \mathrm{MHz}\right)$ spectrum of $\mathbf{4 . 3}$

DM-trytophol data
10 C C13 Experiment
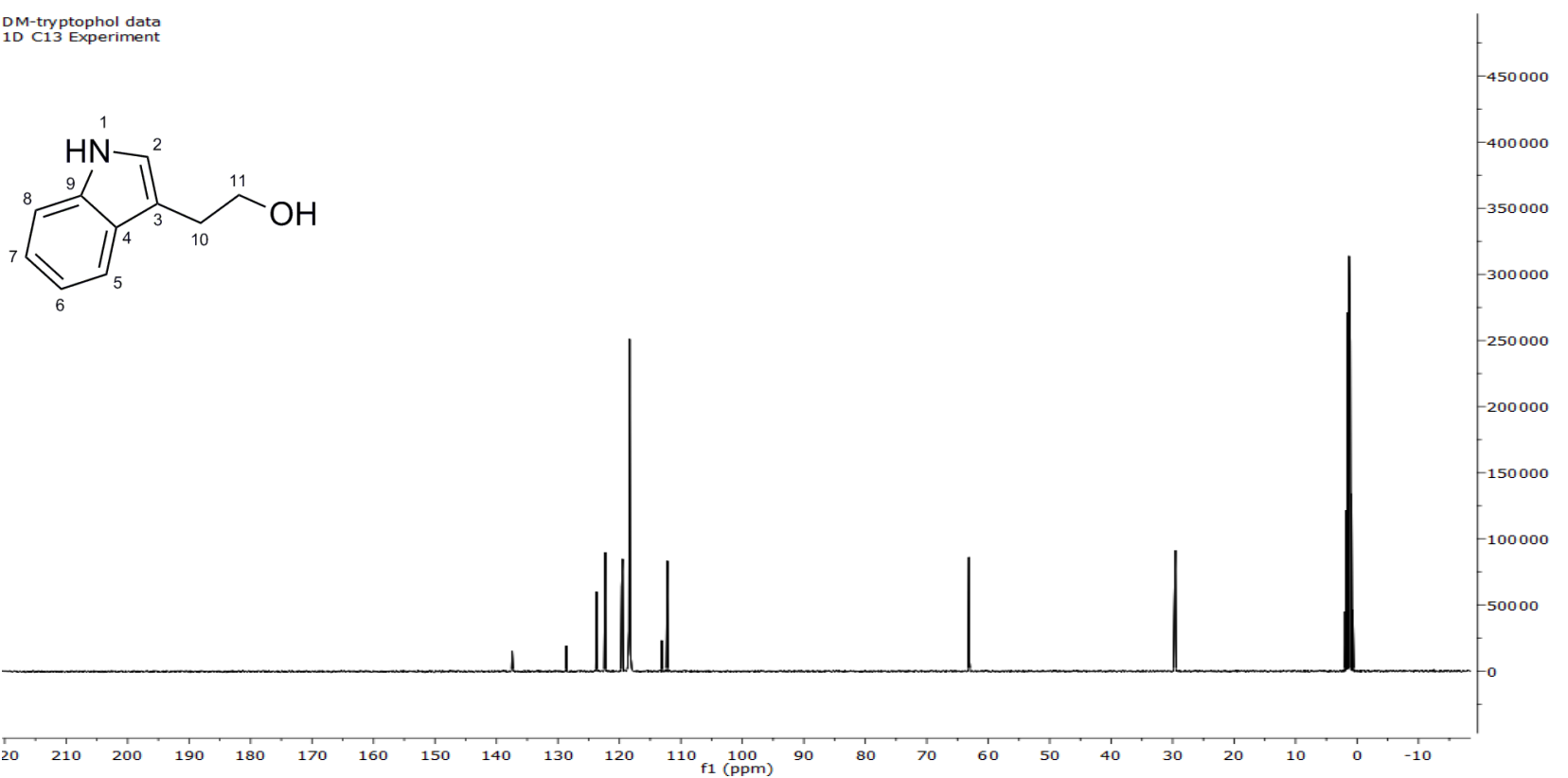
${ }^{1} \mathrm{H}\left(\mathrm{CD}_{3} \mathrm{OD}, 400 \mathrm{MHz}\right)$ and ${ }^{13} \mathrm{C}-\mathrm{NMR}(100 \mathrm{MHz})$ spectra for 4.4

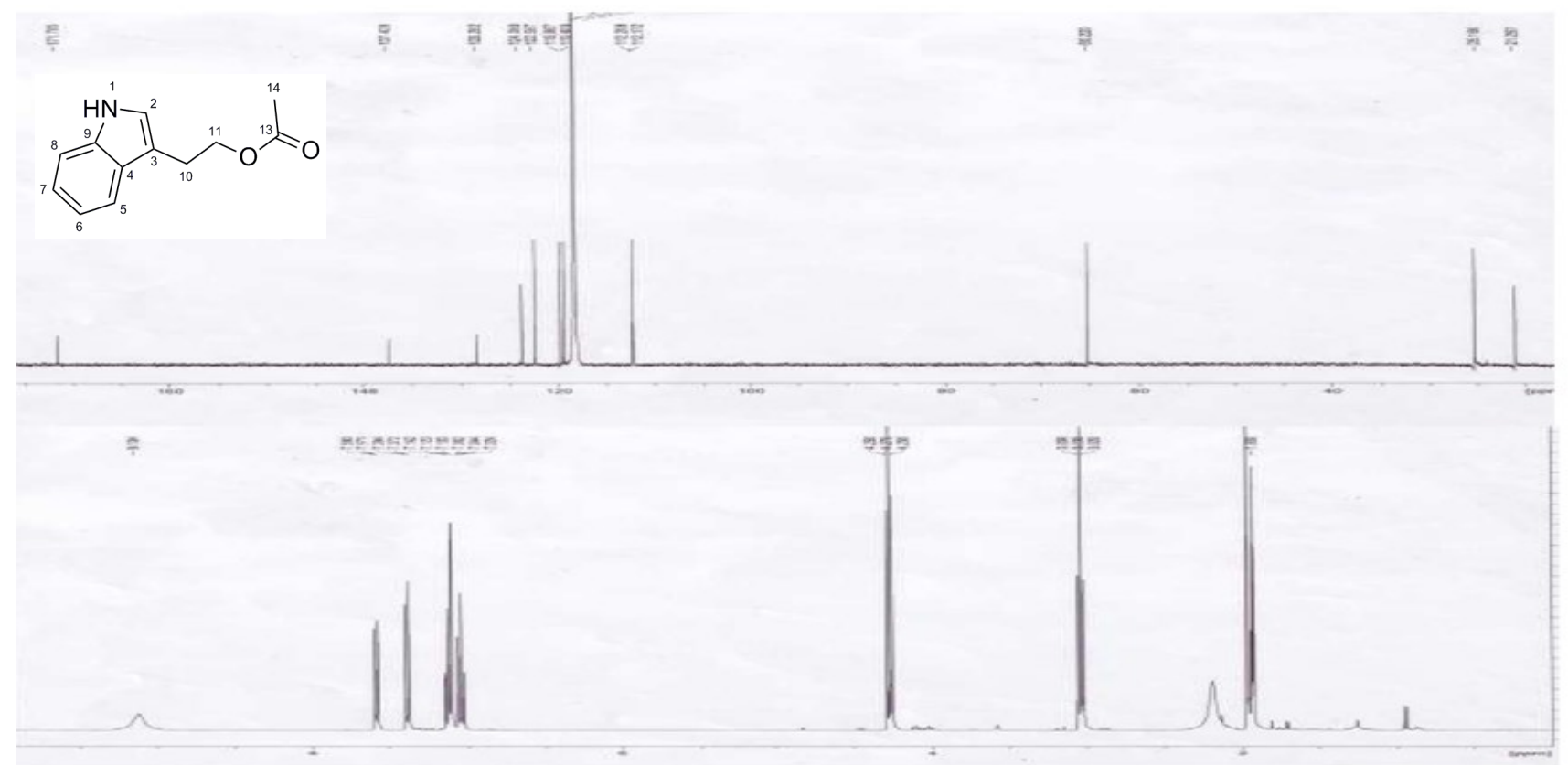

${ }^{1} \mathrm{H}\left(\mathrm{CD}_{3} \mathrm{OD}, 400 \mathrm{MHz}\right)$ and ${ }^{13} \mathrm{C}-\mathrm{NMR}(100 \mathrm{MHz})$ spectra for $\mathbf{4 . 5}$

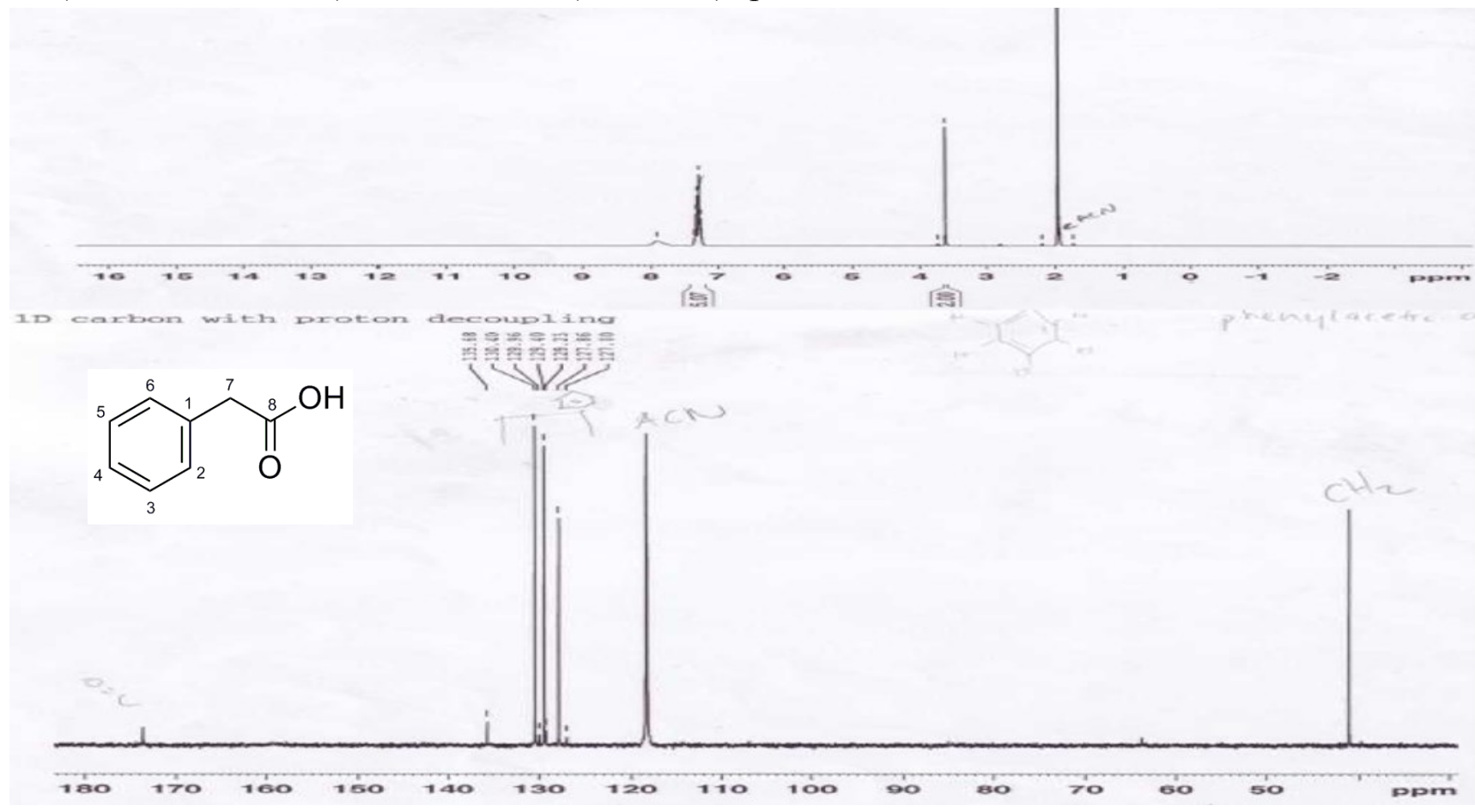


${ }^{1} \mathrm{H}\left(\mathrm{CD}_{3} \mathrm{OD}, 400 \mathrm{MHz}\right)$ and ${ }^{13} \mathrm{C}-\mathrm{NMR}(100 \mathrm{MHz})$ spectra for 4.6

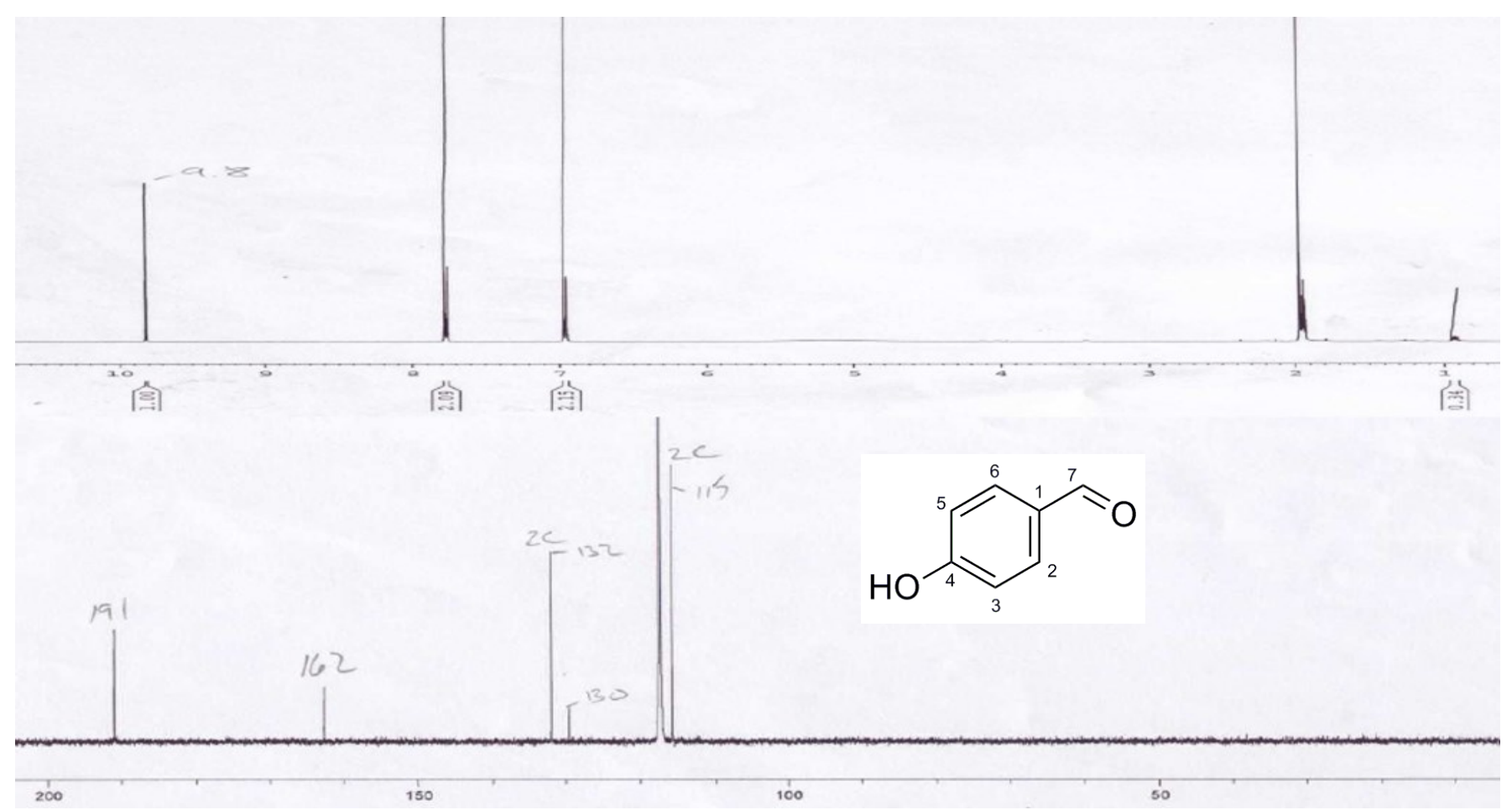




\section{${ }^{1} \mathrm{H}-\mathrm{NMR}\left(\mathrm{CD}_{3} \mathrm{OD}, 400 \mathrm{MHz}\right)$ spectrum of $\mathbf{5 . 1}$}

DM-TC-Fr 7- compound 2- Vertinolide/1 Bruker AVANCE 400 proton

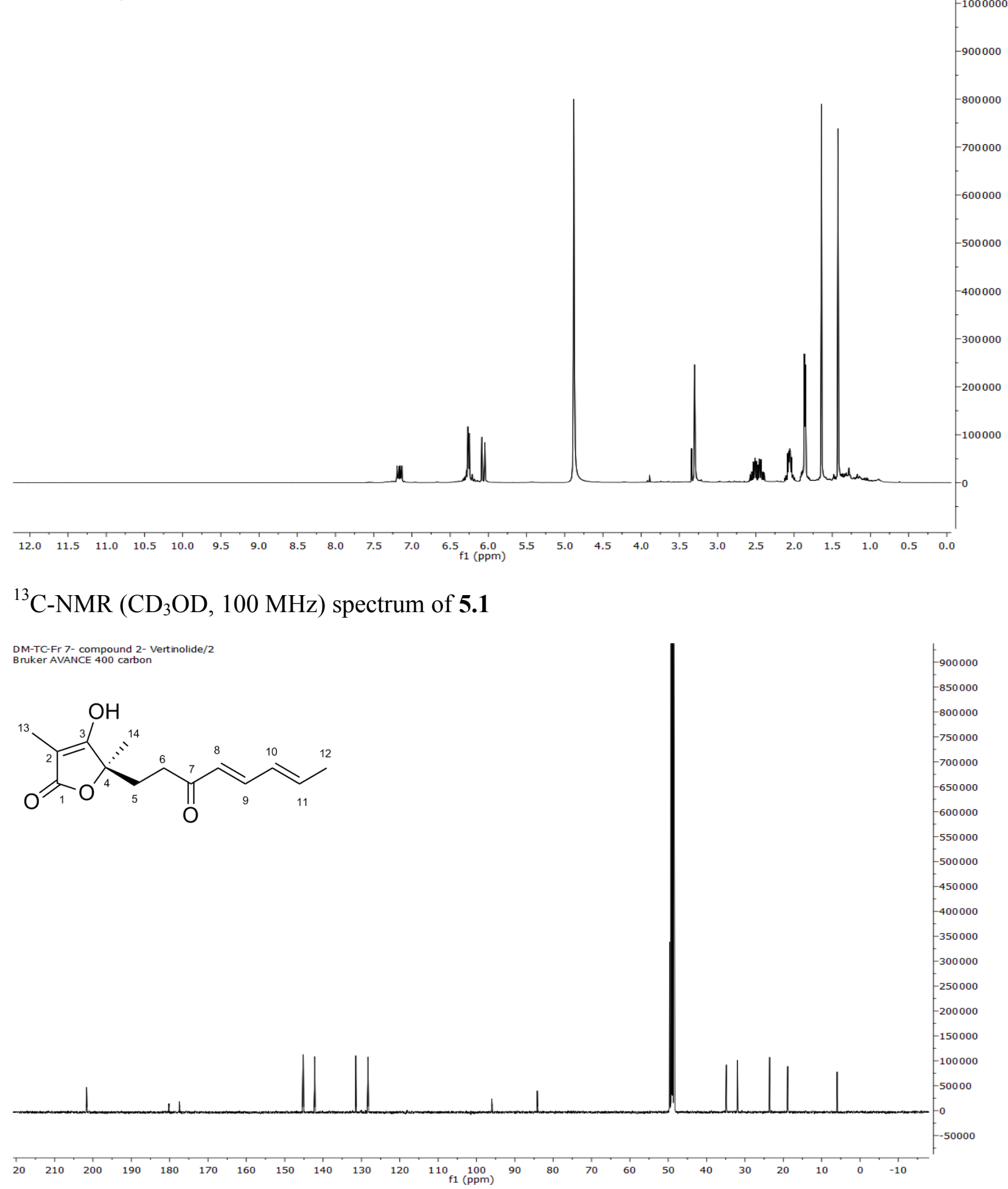


${ }^{1} \mathrm{H}-\mathrm{NMR}\left(\mathrm{CD}_{3} \mathrm{OD}, 400 \mathrm{MHz}\right)$ spectrum of $\mathbf{5 . 2}$

DM-TC-Fr 8-3 maybe/1 Bruker AVANCE 400 proton

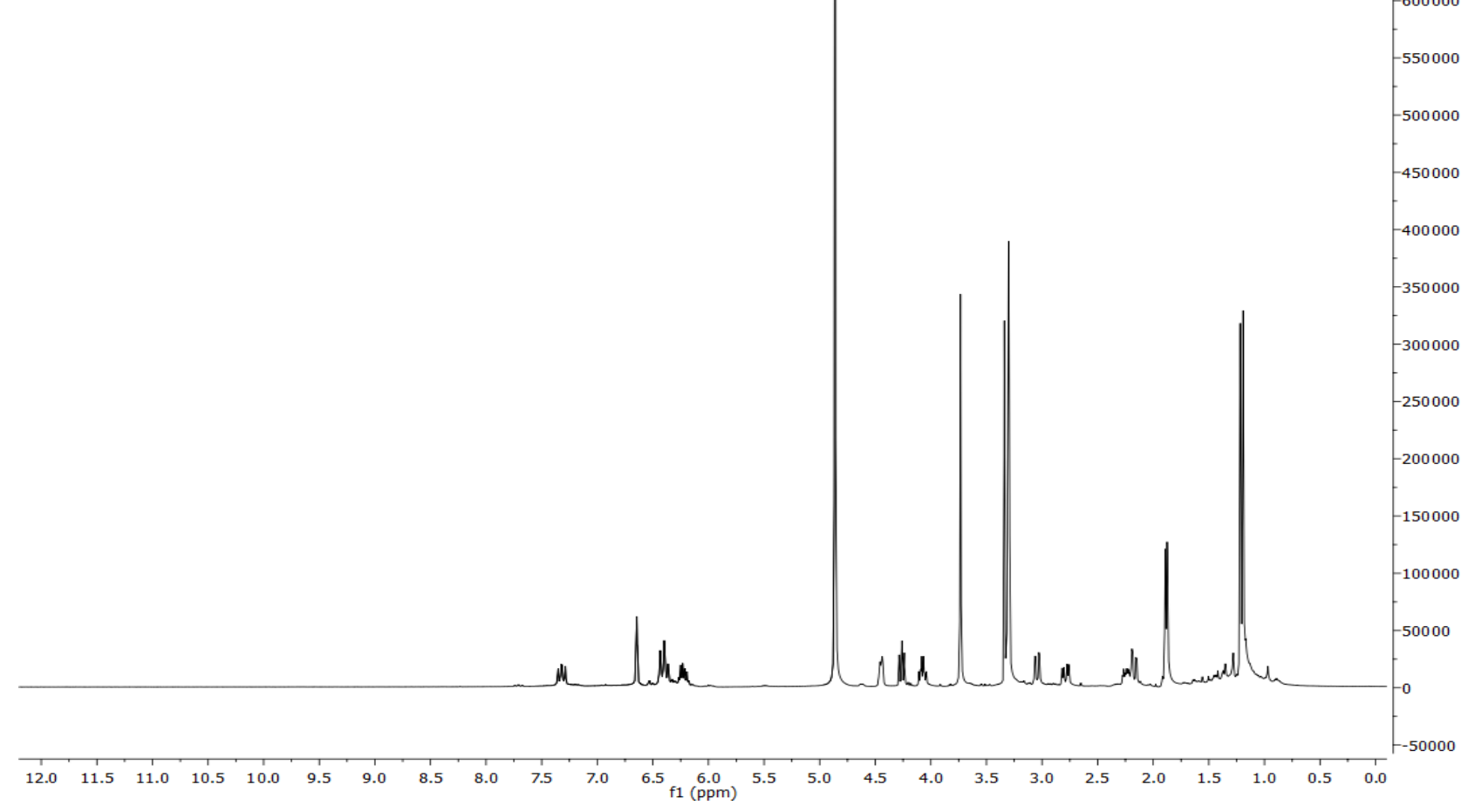

${ }^{13} \mathrm{C}-\mathrm{NMR}\left(\mathrm{CD}_{3} \mathrm{OD}, 100 \mathrm{MHz}\right)$ spectrum of $\mathbf{5 . 2}$

DM-TC-Fr 8-3 maybe/2

Bruker AVANCE 400 carbon
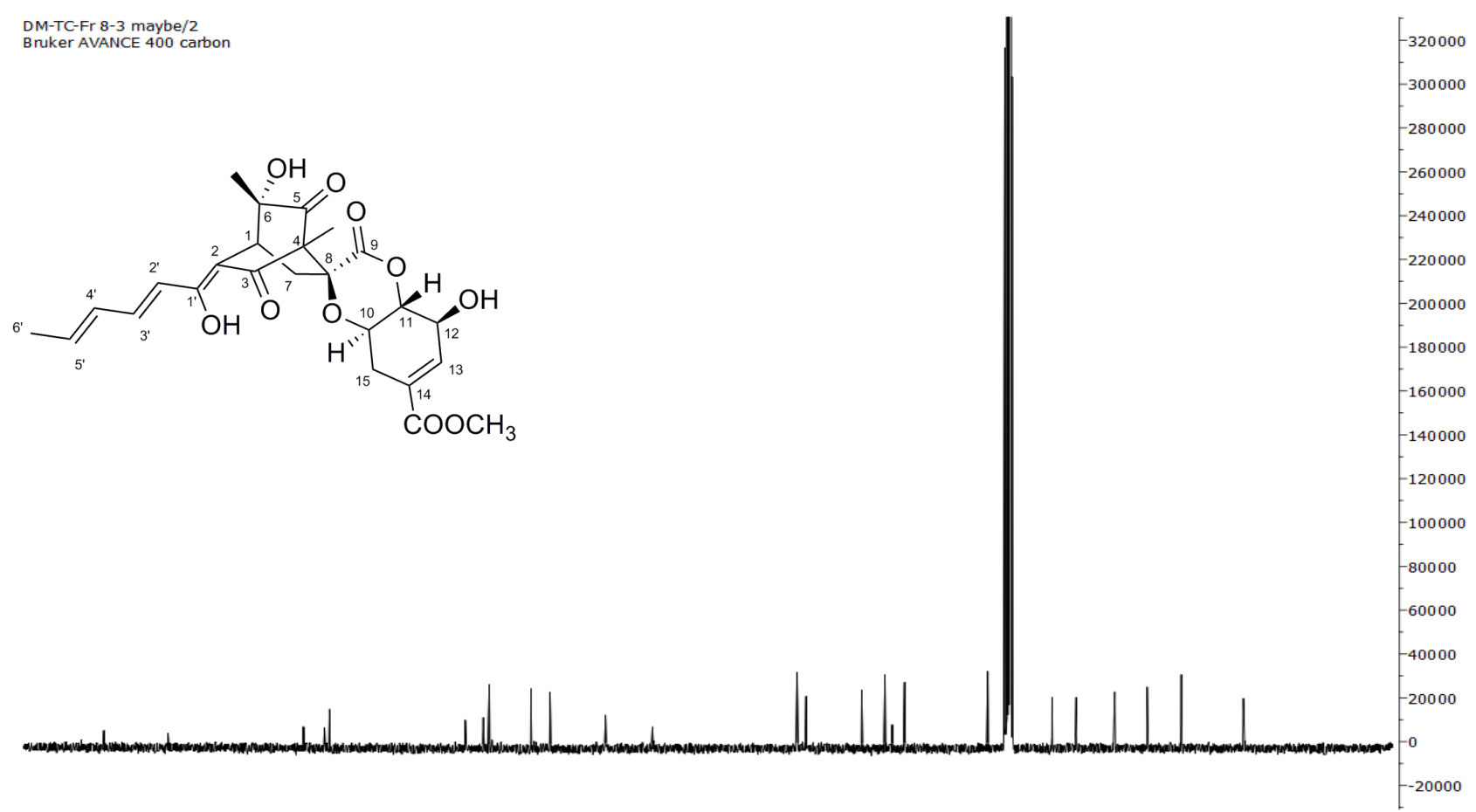

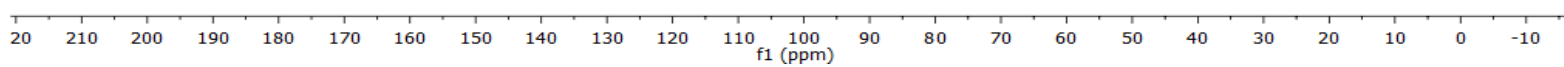


${ }^{1} \mathrm{H}-\mathrm{NMR}\left(\mathrm{CD}_{3} \mathrm{OD}, 400 \mathrm{MHz}\right)$ spectrum of $\mathbf{5 . 3}$

DM-TC-Fr 8-2/1

Bruker AVANCE 400 proton

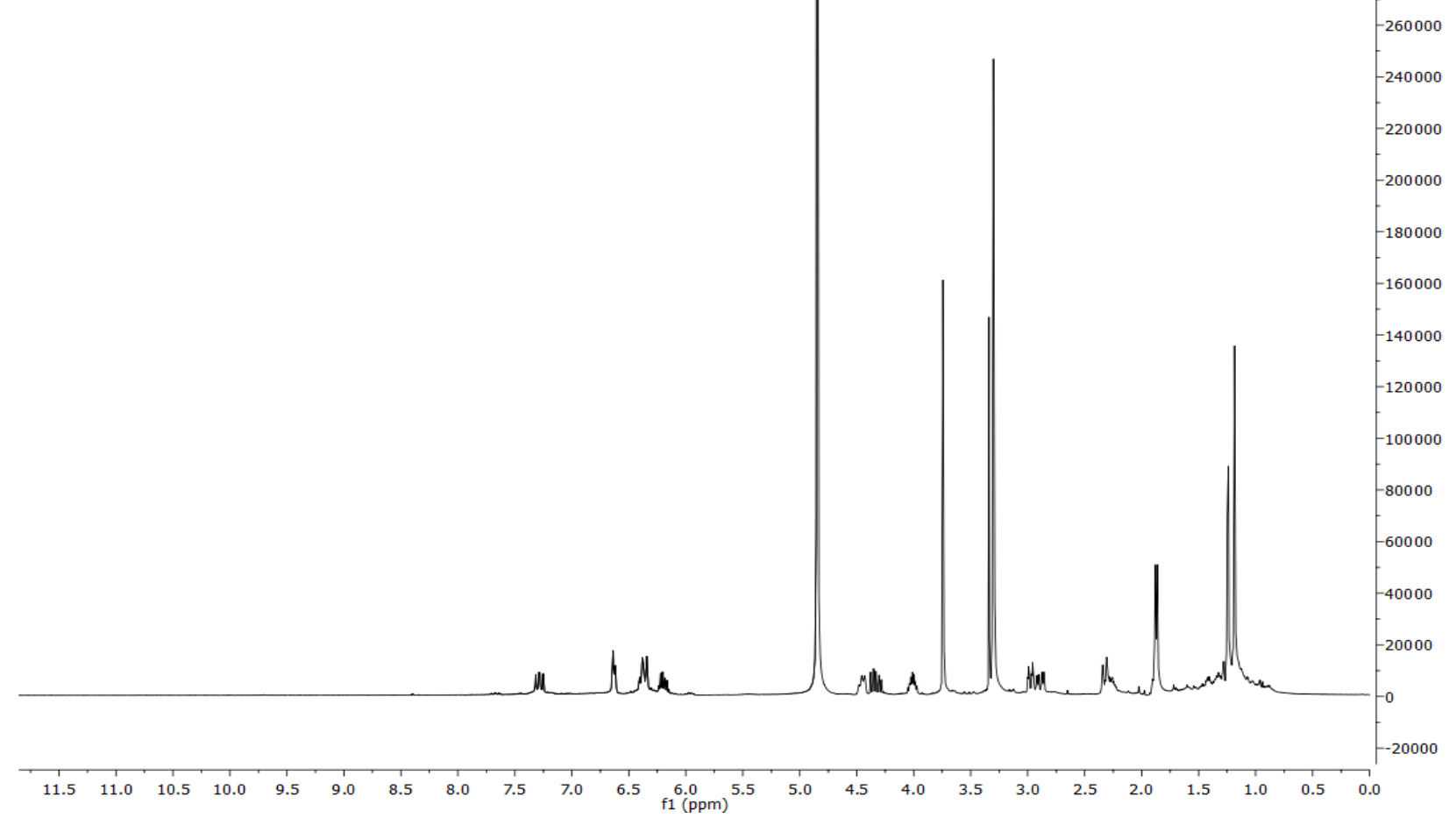

${ }^{13} \mathrm{C}-\mathrm{NMR}\left(\mathrm{CD}_{3} \mathrm{OD}, 100 \mathrm{MHz}\right)$ spectrum of $\mathbf{5 . 3}$
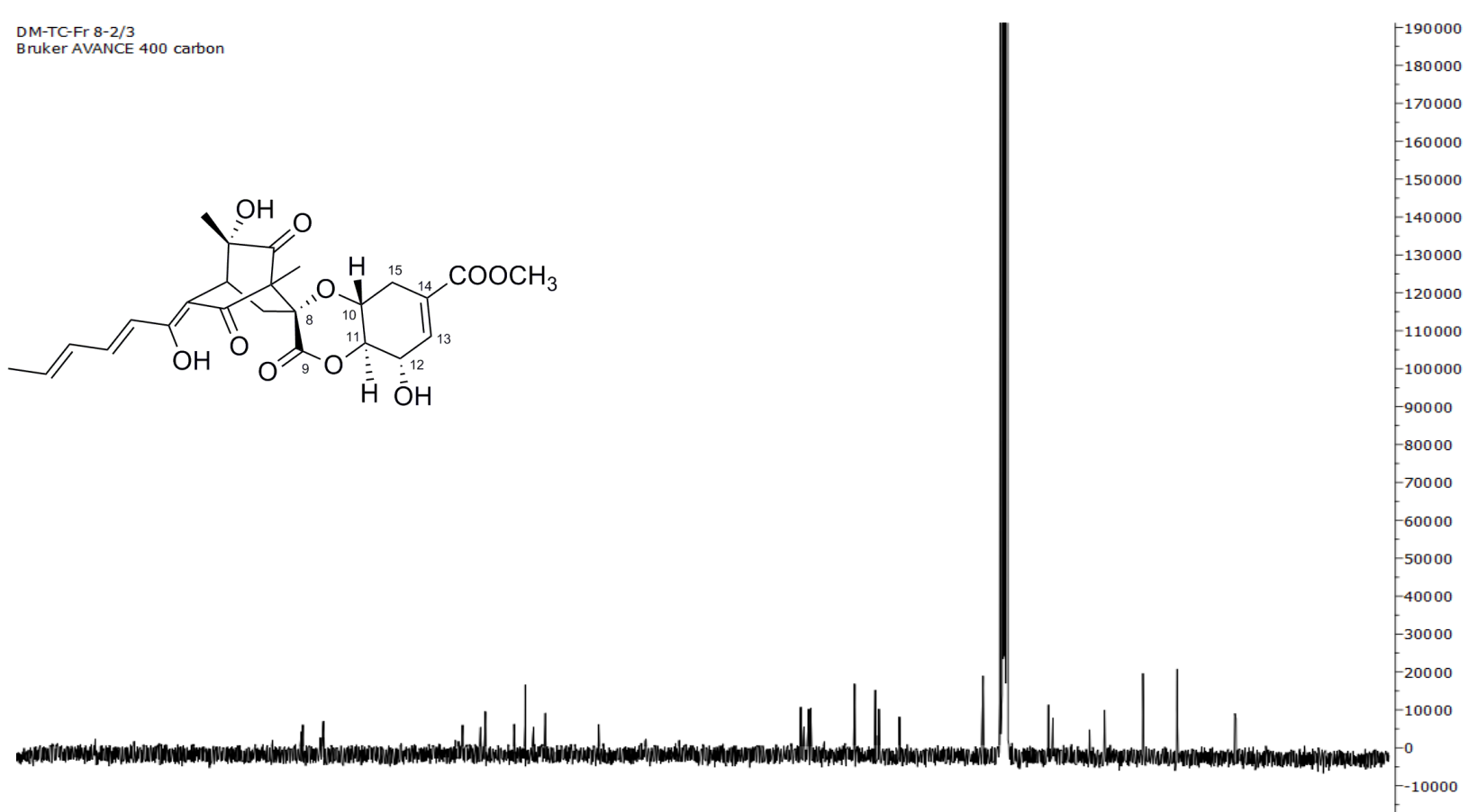

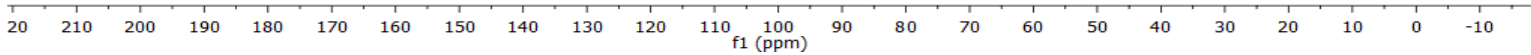


${ }^{1} \mathrm{H}-\mathrm{NMR}\left(\mathrm{CD}_{3} \mathrm{OD}, 400 \mathrm{MHz}\right)$ spectrum of $\mathbf{5 . 4}$

DM-TC-Fr7-3/2

Bruker AVANCE 400 proton

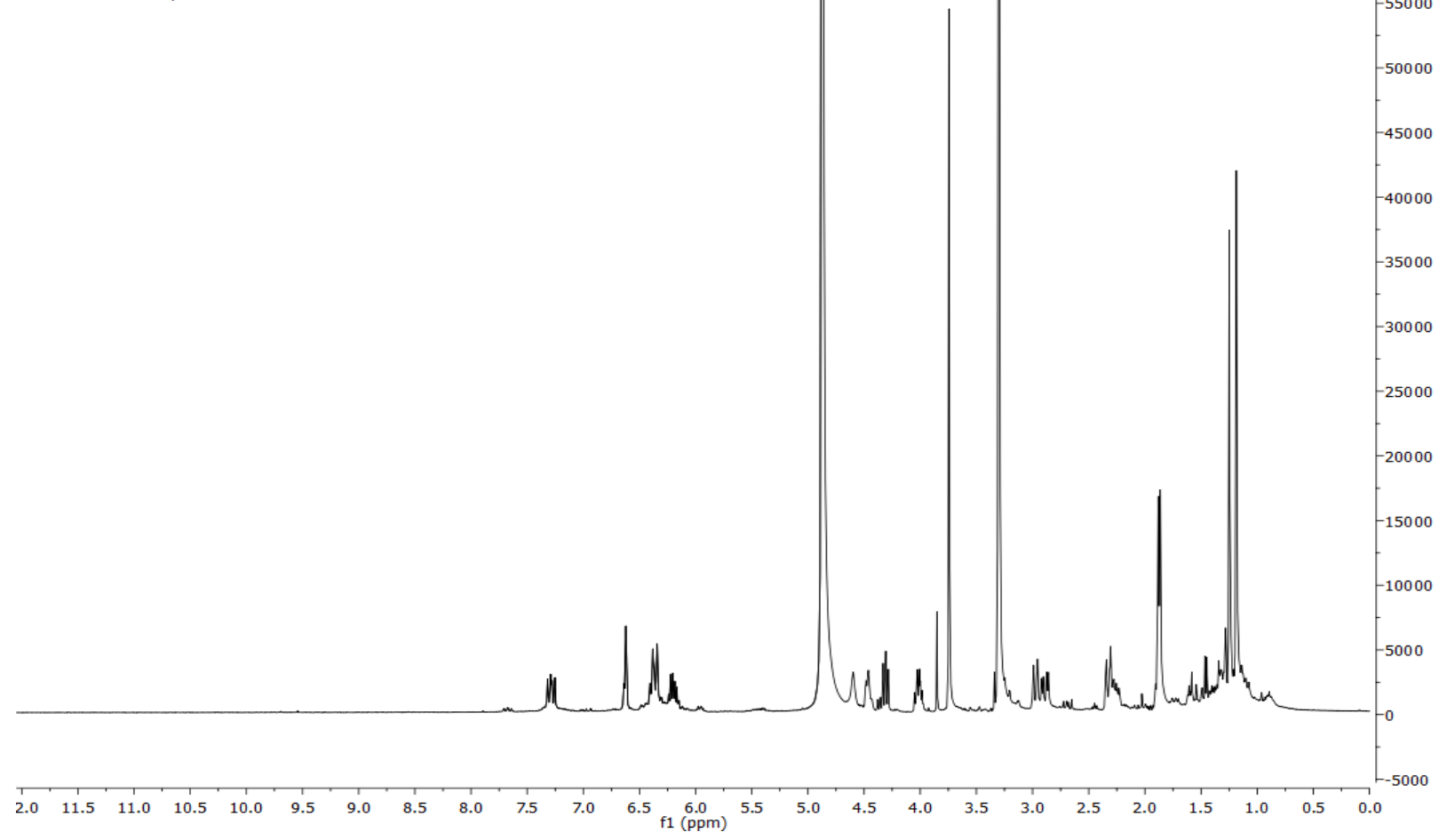

${ }^{13} \mathrm{C}-\mathrm{NMR}\left(\mathrm{CD}_{3} \mathrm{OD}, 100 \mathrm{MHz}\right)$ spectrum of $\mathbf{5 . 4}$

DM-TC-Fr7-3/3

Bruker AVANCE 400 carbon
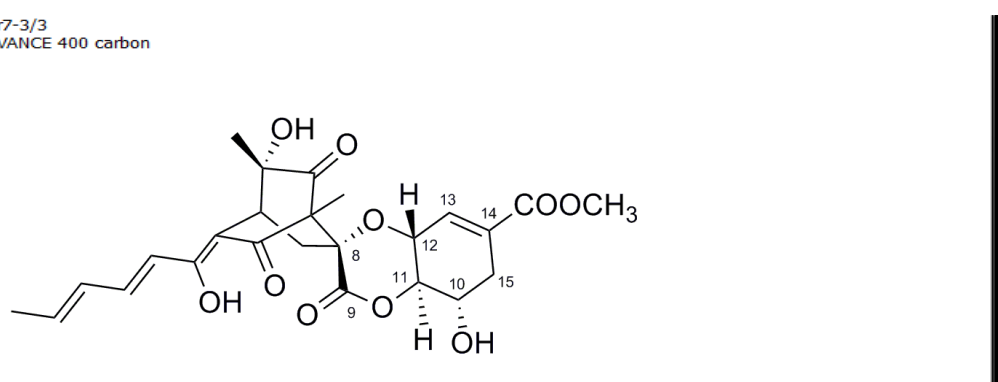
${ }^{1} \mathrm{H}-\mathrm{NMR}\left(\mathrm{CD}_{3} \mathrm{OD}, 400 \mathrm{MHz}\right)$ spectrum of $\mathbf{5 . 5}$

DM-TC-Fr8-4/1

Bruker AVANCE 400 proton

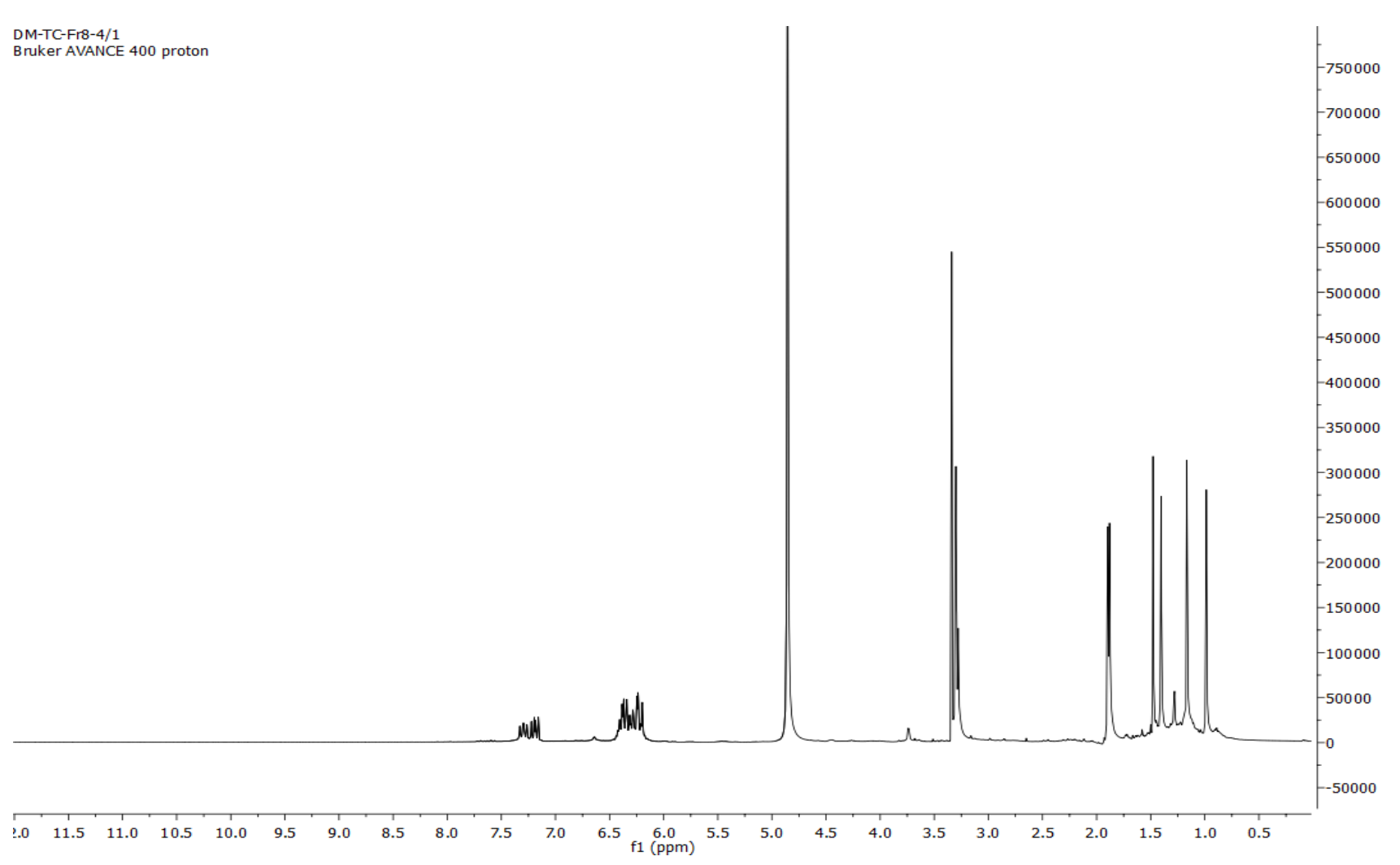

${ }^{13} \mathrm{C}-\mathrm{NMR}\left(\mathrm{CD}_{3} \mathrm{OD}, 100 \mathrm{MHz}\right)$ spectrum of $\mathbf{5 . 5}$

DM-TC-Fr8-4/2

Bruker AVANCE 400 carbon

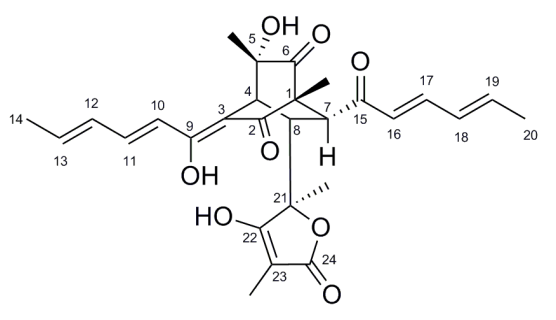


${ }^{1} \mathrm{H}-\mathrm{NMR}\left(\mathrm{CD}_{3} \mathrm{OD}, 400 \mathrm{MHz}\right)$ spectrum of $\mathbf{5 . 6}$

DM-TC-Fr 7-6 (498 Da)/1 Bruker AVANCE 400 proton

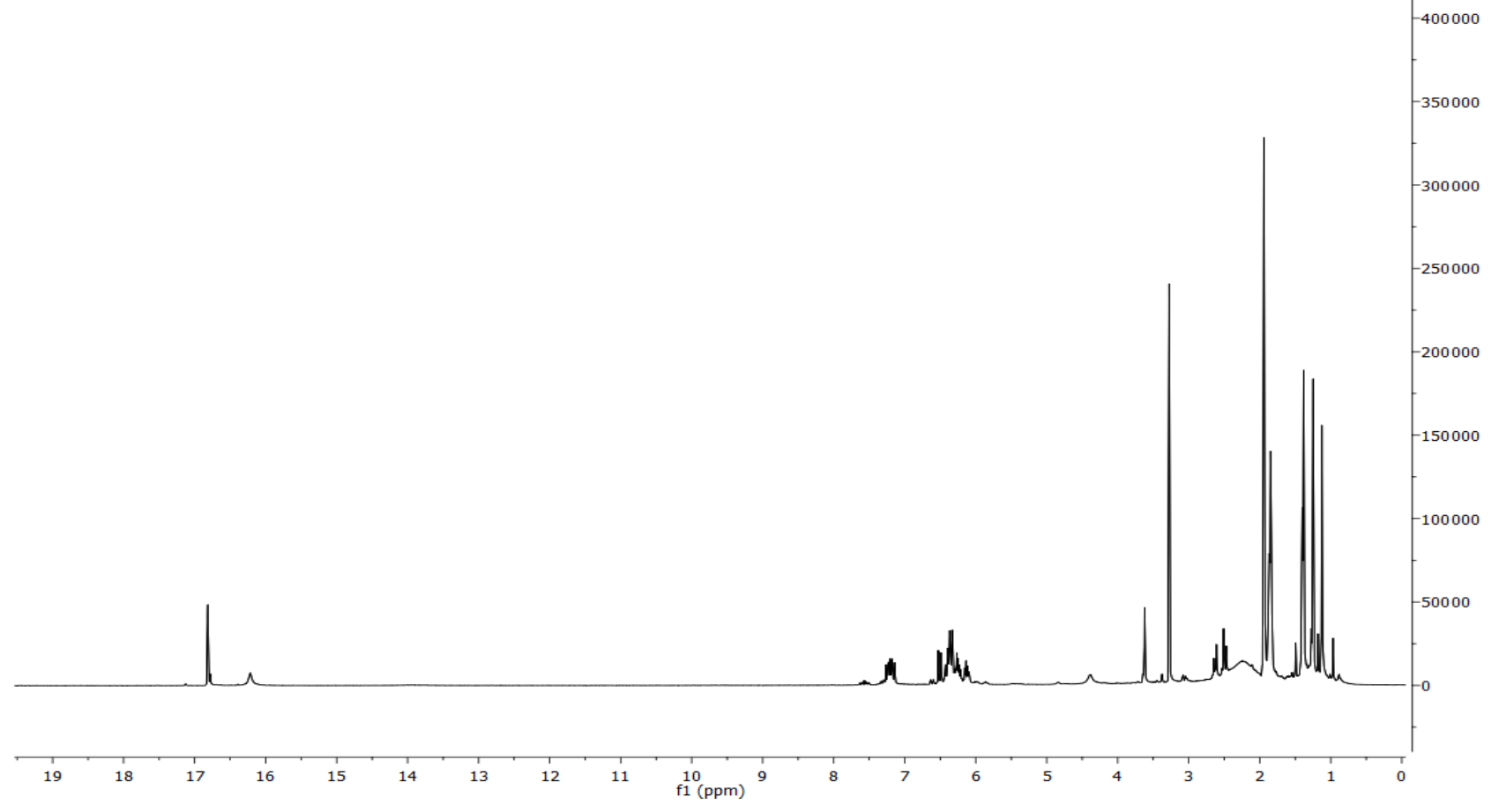

${ }^{13} \mathrm{C}-\mathrm{NMR}\left(\mathrm{CD}_{3} \mathrm{OD}, 100 \mathrm{MHz}\right)$ spectrum of $\mathbf{5 . 6}$

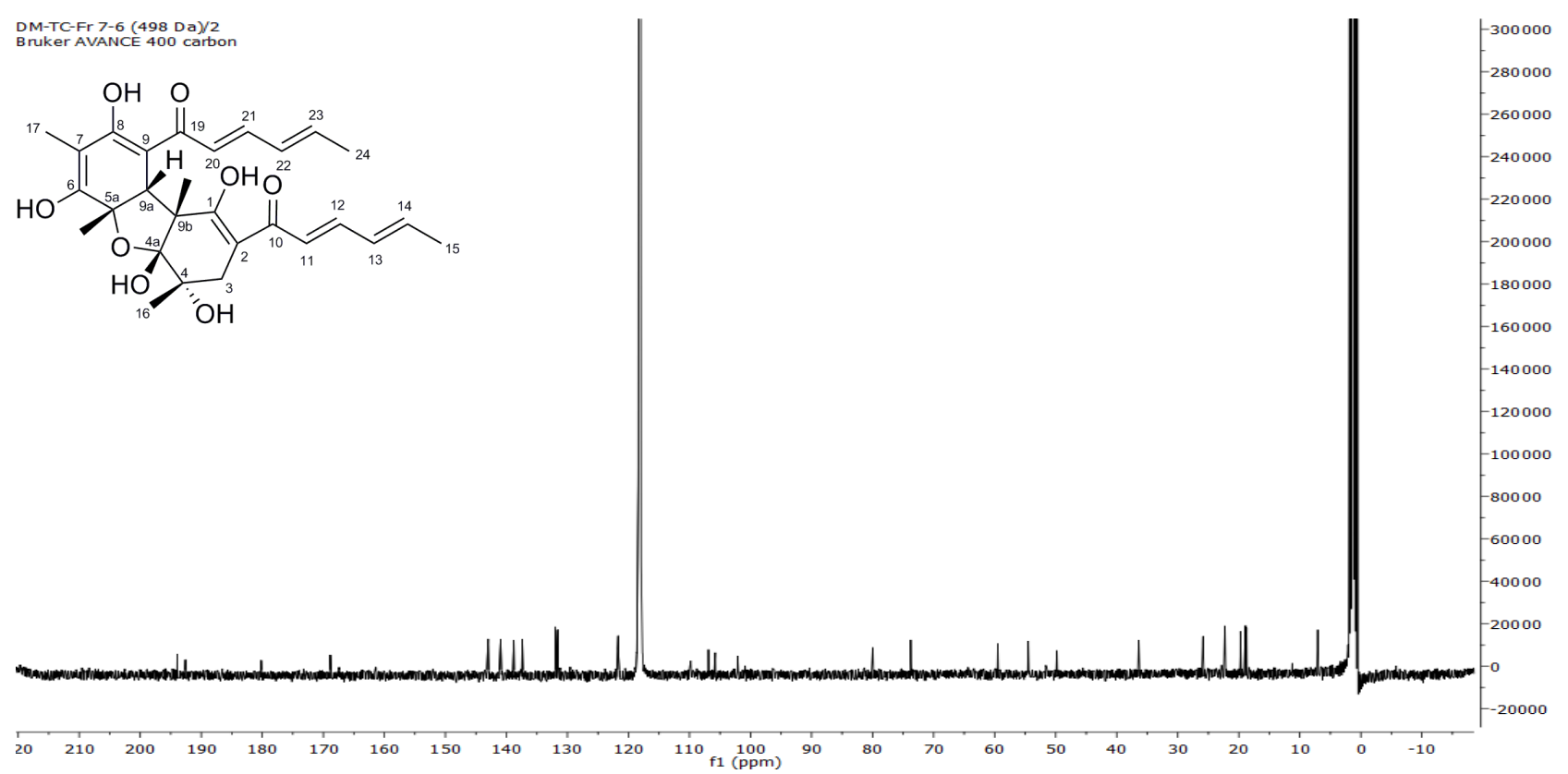




\section{${ }^{1} \mathrm{H}-\mathrm{NMR}\left(\mathrm{CD}_{3} \mathrm{OD}, 400 \mathrm{MHz}\right)$ spectrum of $\mathbf{5 . 7}$}

DM-TK- 284 MW A/1

Bruker AVANCE 400 proton

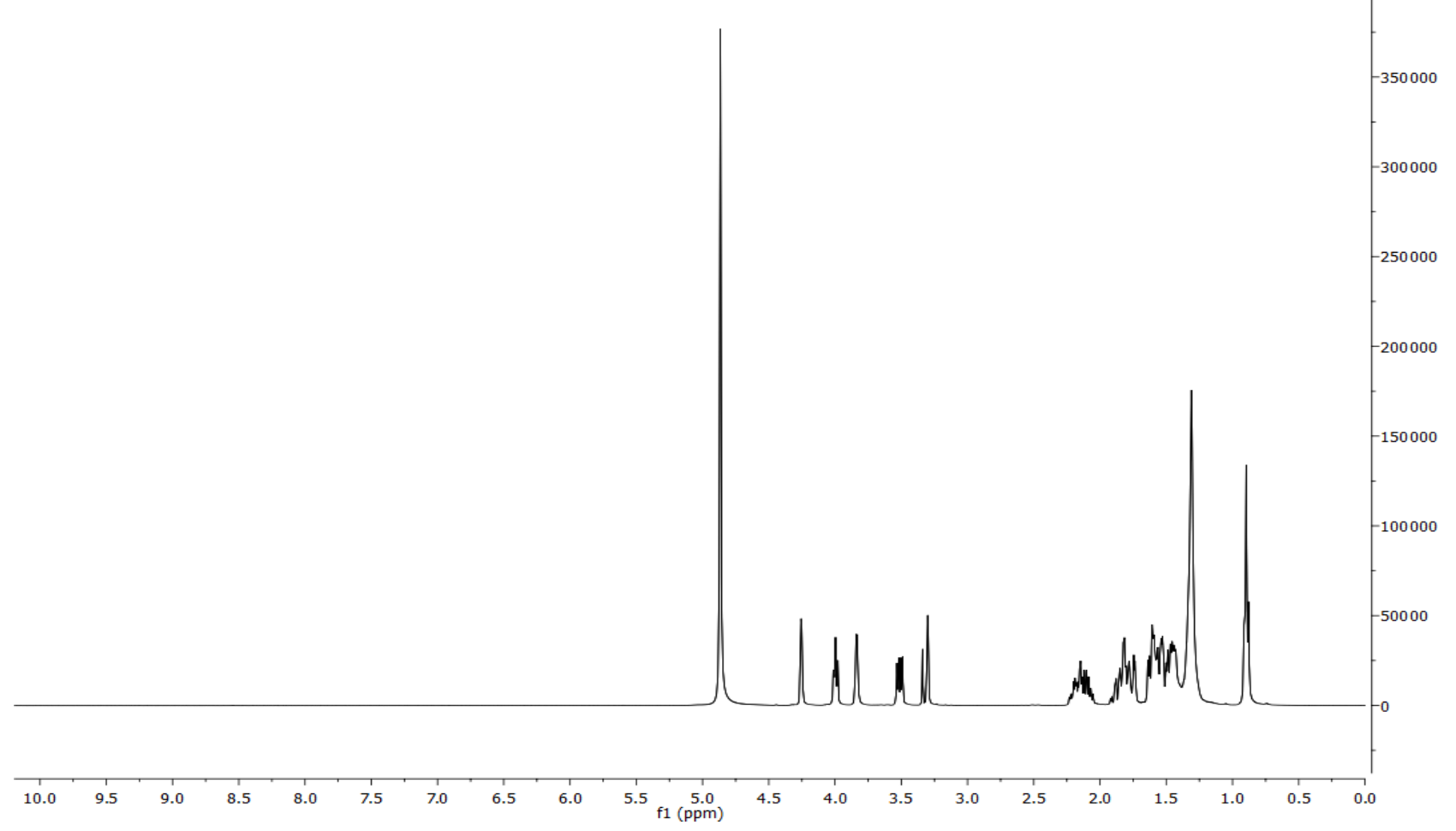

${ }^{13} \mathrm{C}-\mathrm{NMR}\left(\mathrm{CD}_{3} \mathrm{OD}, 100 \mathrm{MHz}\right)$ spectrum of $\mathbf{5 . 7}$

DM-TK- 284 MW A/2
Bruker AVANCE 400 carbon<smiles>CCCCCCC1O[C@@]23O[C@@H]1CC[C@H]2[C@H](O)CC[C@H]3O</smiles>

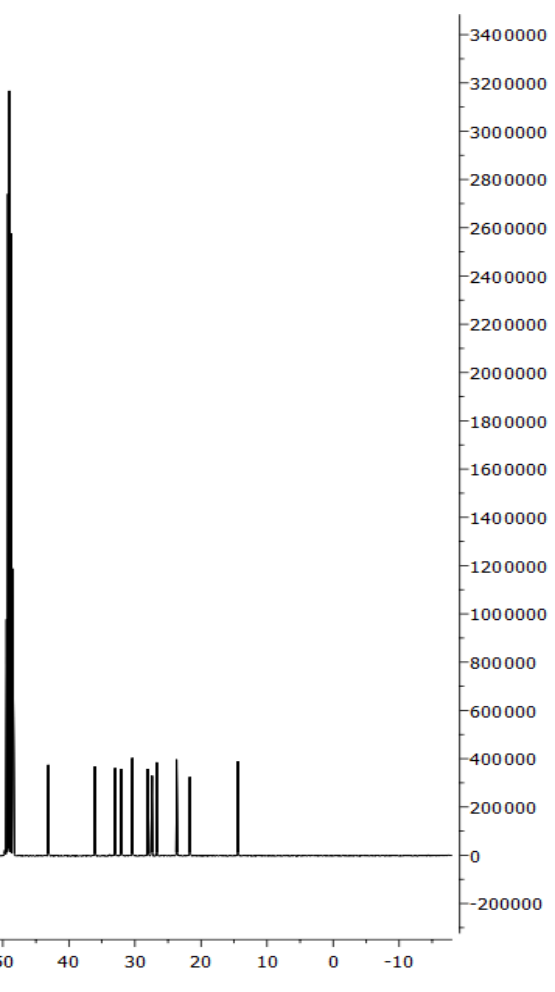

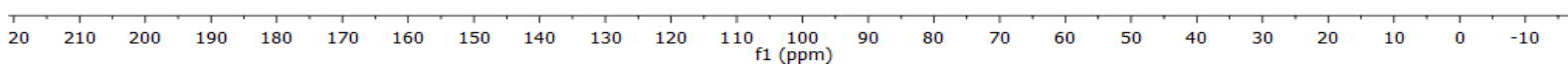


${ }^{1} \mathrm{H}-\mathrm{NMR}\left(\mathrm{CD}_{3} \mathrm{OD}, 400 \mathrm{MHz}\right)$ spectrum of $\mathbf{5 . 8}$

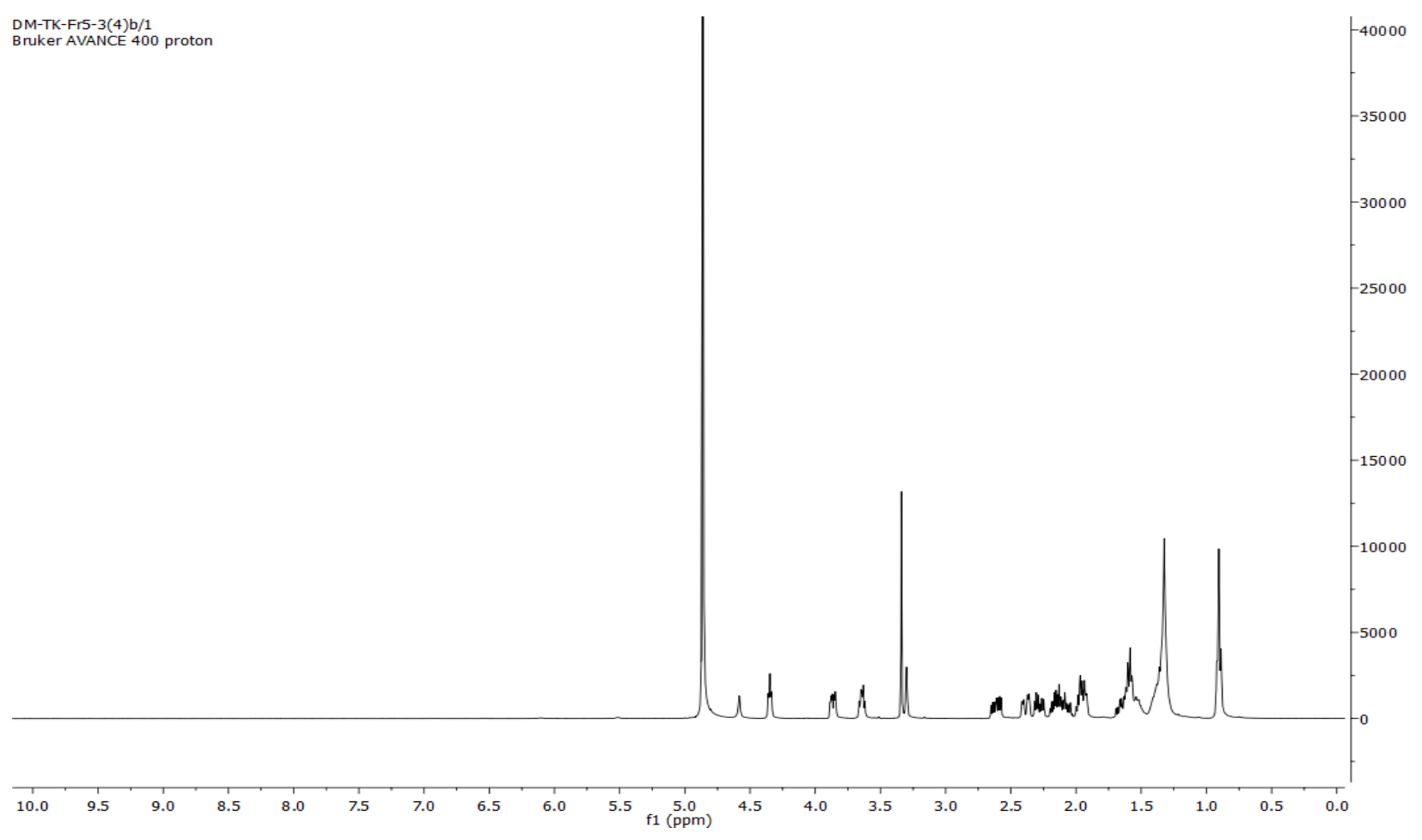

${ }^{13} \mathrm{C}-\mathrm{NMR}\left(\mathrm{CD}_{3} \mathrm{OD}, 100 \mathrm{MHz}\right)$ spectrum of $\mathbf{5 . 8}$

DM-TK-Fr5-3(4)b/2

Bruker AVANCE 400 carbon
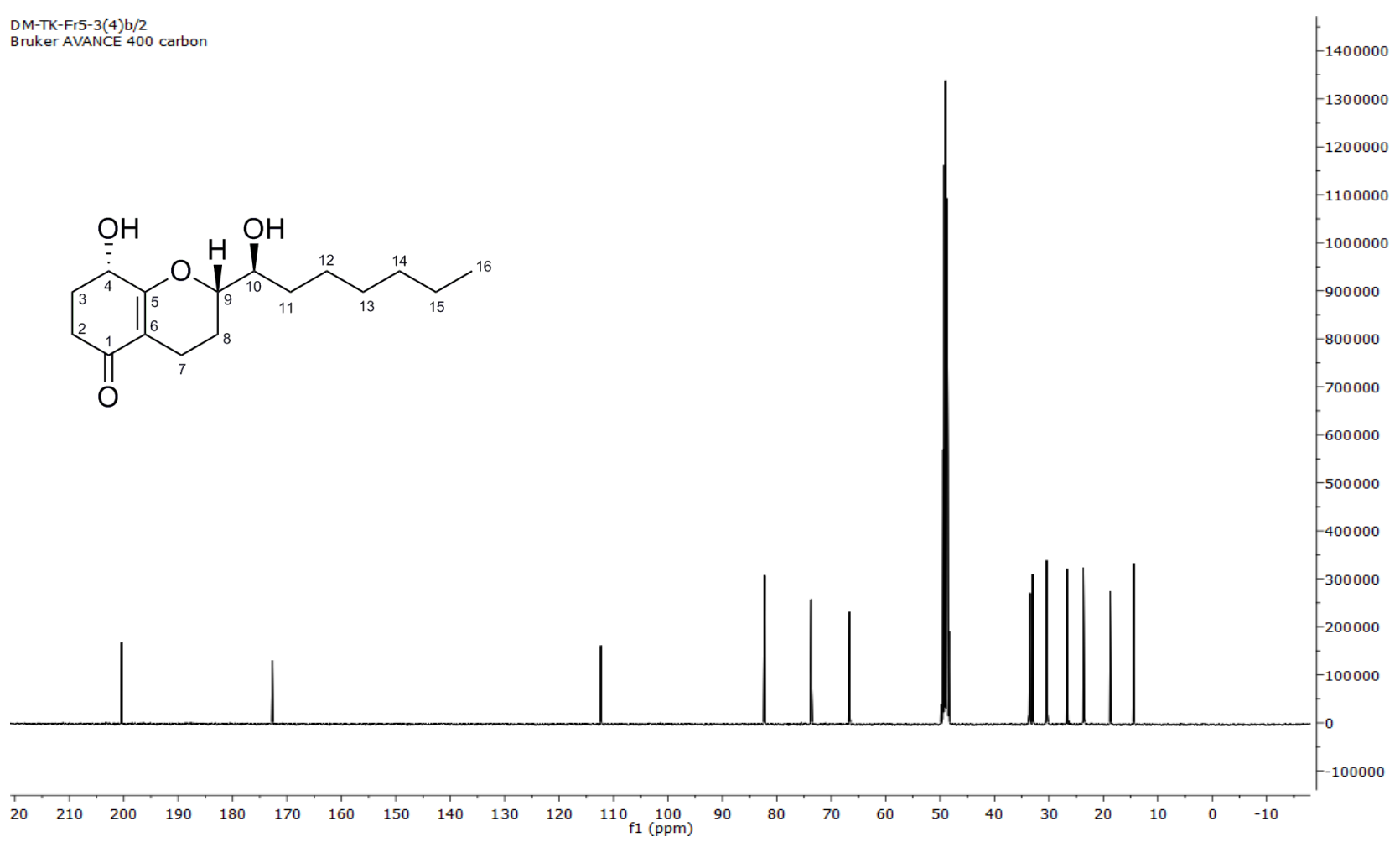

256 
${ }^{1} \mathrm{H}-\mathrm{NMR}\left(\mathrm{CD}_{3} \mathrm{OD}, 400 \mathrm{MHz}\right)$ spectrum of $\mathbf{5 . 9}$

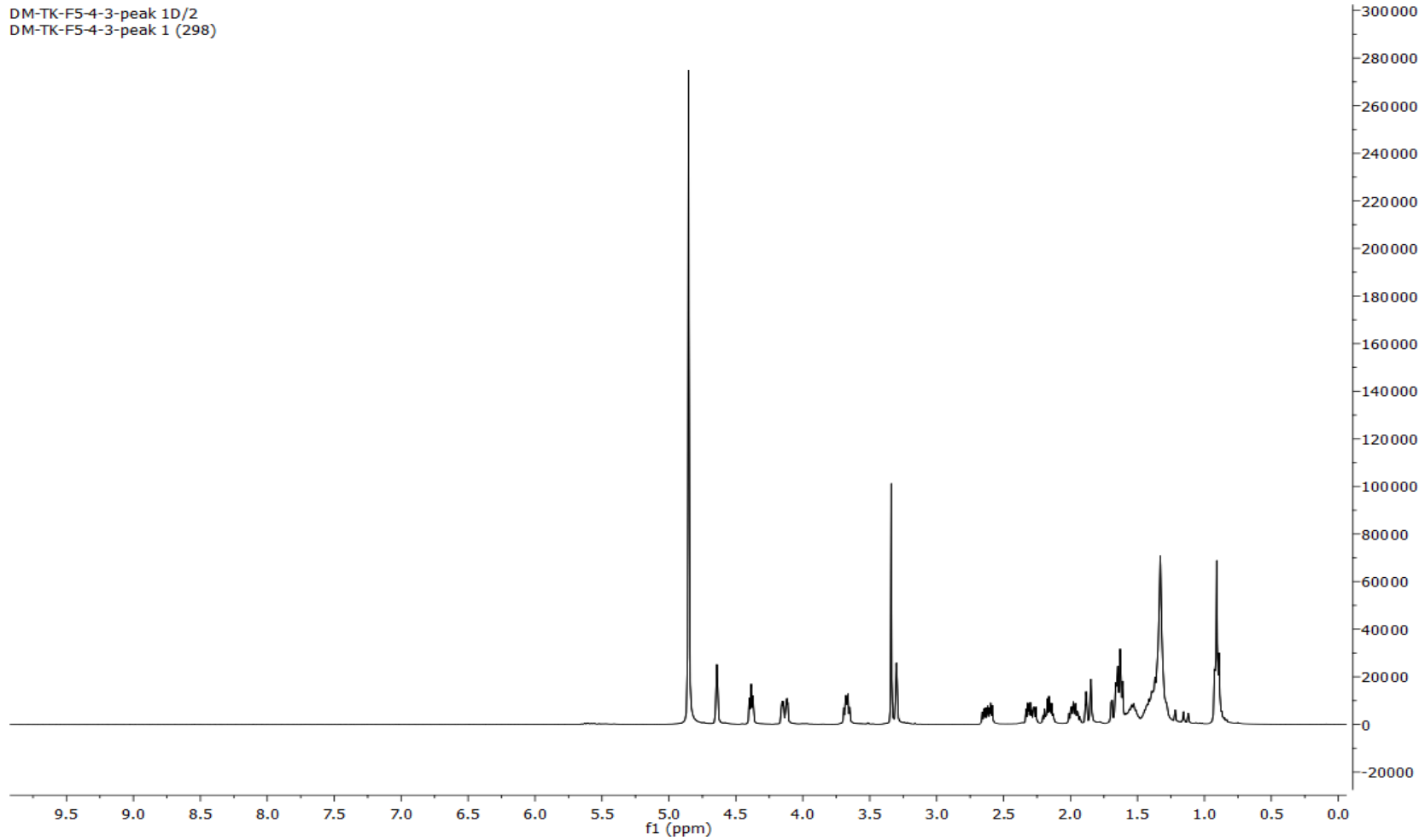

${ }^{13} \mathrm{C}-\mathrm{NMR}\left(\mathrm{CD}_{3} \mathrm{OD}, 100 \mathrm{MHz}\right)$ spectrum of $\mathbf{5 . 9}$

DM-TK-F5-4-3-peak 1D/3
Bruker AVANCE 400 carbon<smiles>CCCCCC[C@@H](O)[C@H]1C[C@@H](O)C2=C(O1)[C@H](O)CCC2=O</smiles>

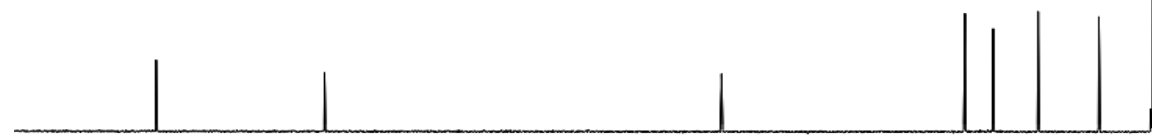




\section{${ }^{1} \mathrm{H}-\mathrm{NMR}\left(\mathrm{CD}_{3} \mathrm{OD}, 400 \mathrm{MHz}\right)$ spectrum of $\mathbf{5 . 1 0}$}

DM-TK-Fr5-4-3-peak 2F/1 Bruker AVANCE 400 proto

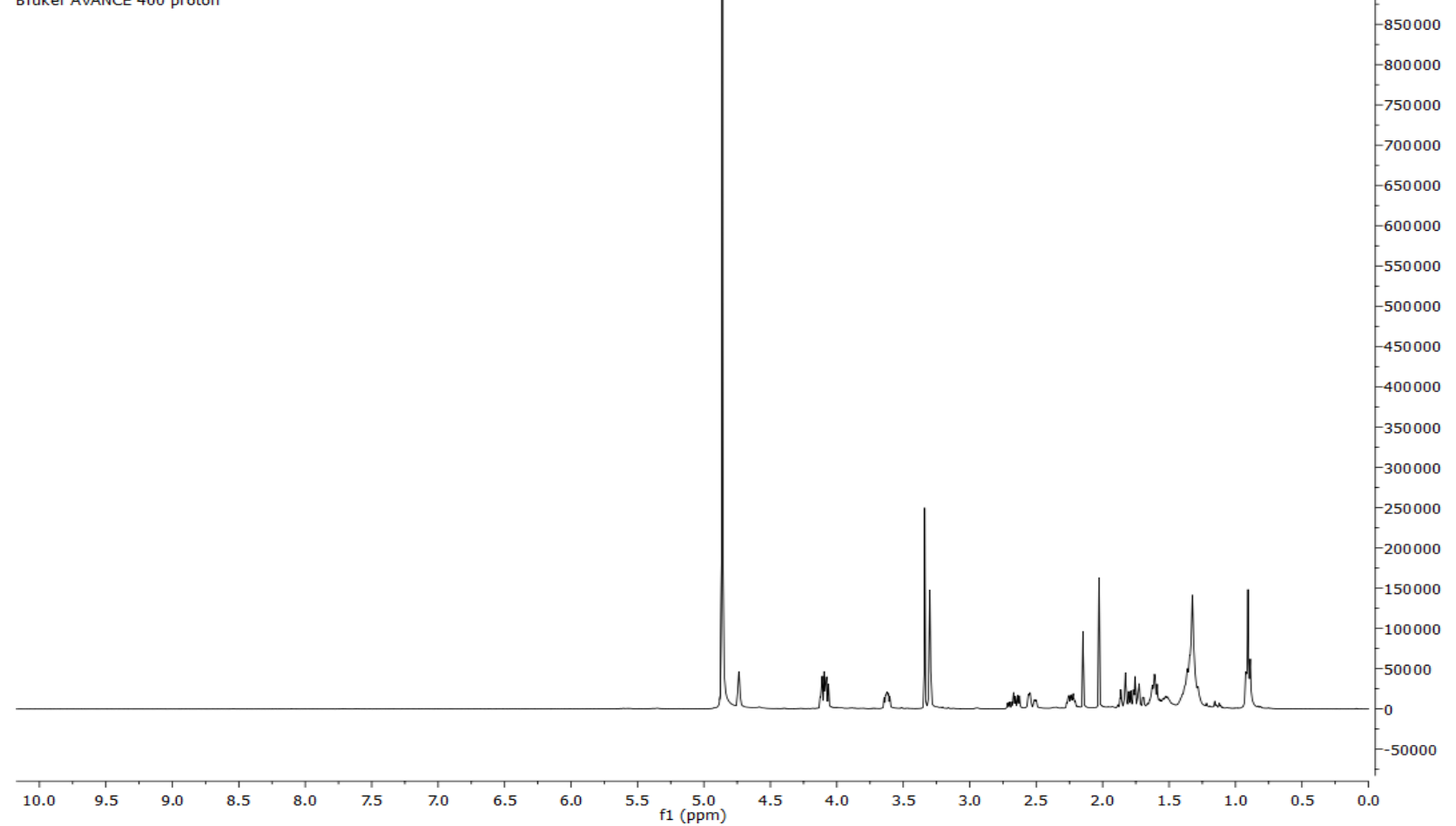

${ }^{13} \mathrm{C}-\mathrm{NMR}\left(\mathrm{CD}_{3} \mathrm{OD}, 100 \mathrm{MHz}\right)$ spectrum of $\mathbf{5 . 1 0}$

DM-TK-Fr5-4-3-peak 2F/2 Bruker AVANCE 400 carbon<smiles>CCCCCC[C@@H](O)[C@H]1C[C@@H](O)C2=C(CC[C@@H](O)C2=O)O1</smiles>

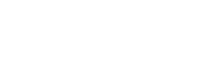

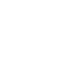


${ }^{1} \mathrm{H}-\mathrm{NMR}\left(\mathrm{CD}_{3} \mathrm{OD}, 400 \mathrm{MHz}\right)$ spectrum of $\mathbf{5 . 1 1}$

DM-TA-Fr 3-HPLC 5-major compound/1

Bruker AVANCE 400 proton

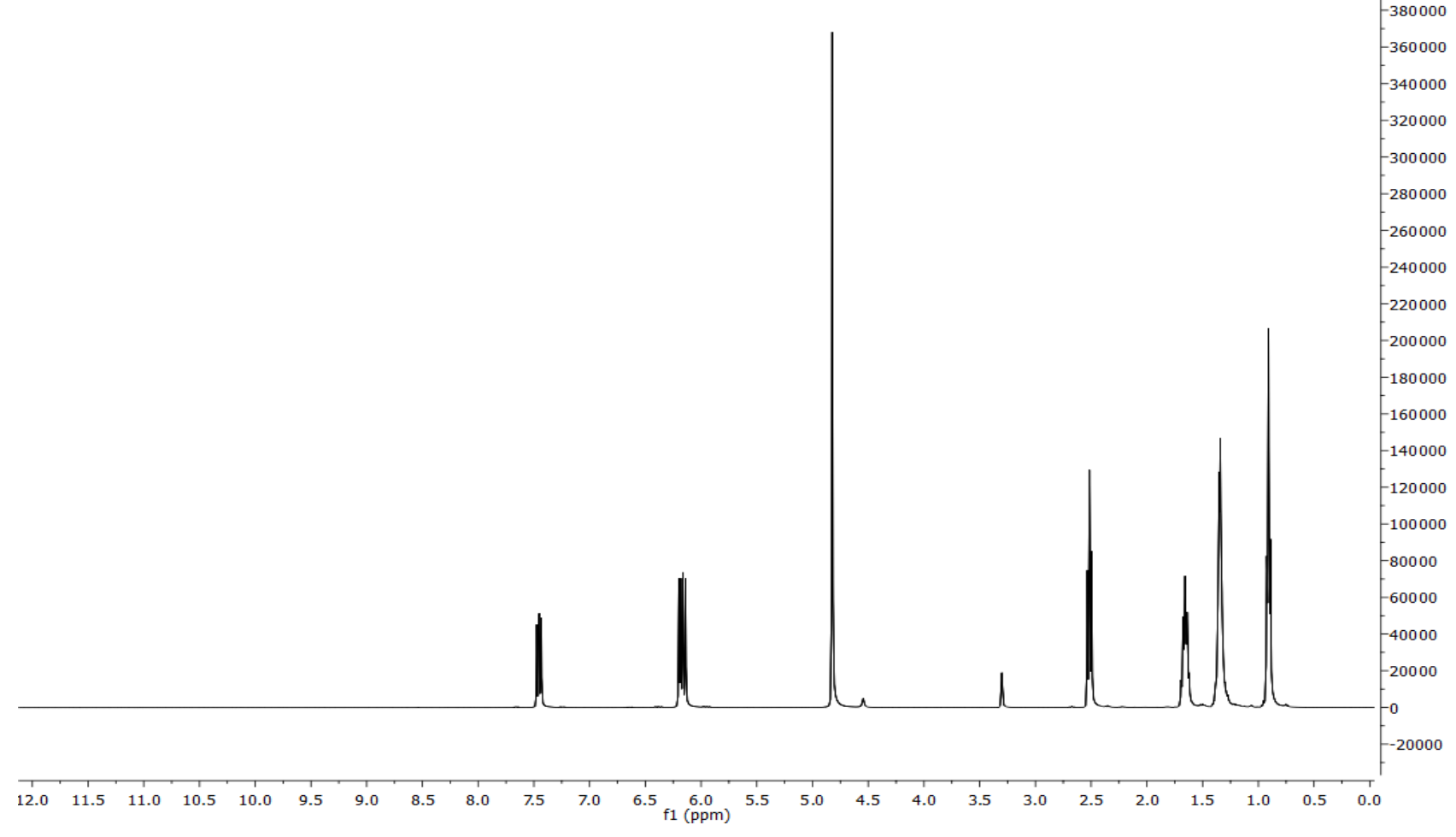

${ }^{13} \mathrm{C}-\mathrm{NMR}\left(\mathrm{CD}_{3} \mathrm{OD}, 100 \mathrm{MHz}\right)$ spectrum of $\mathbf{5 . 1 1}$

DM-TA-Fr 3-HPLC 5-major compound/2

Bruker AVANCE 400 carbon
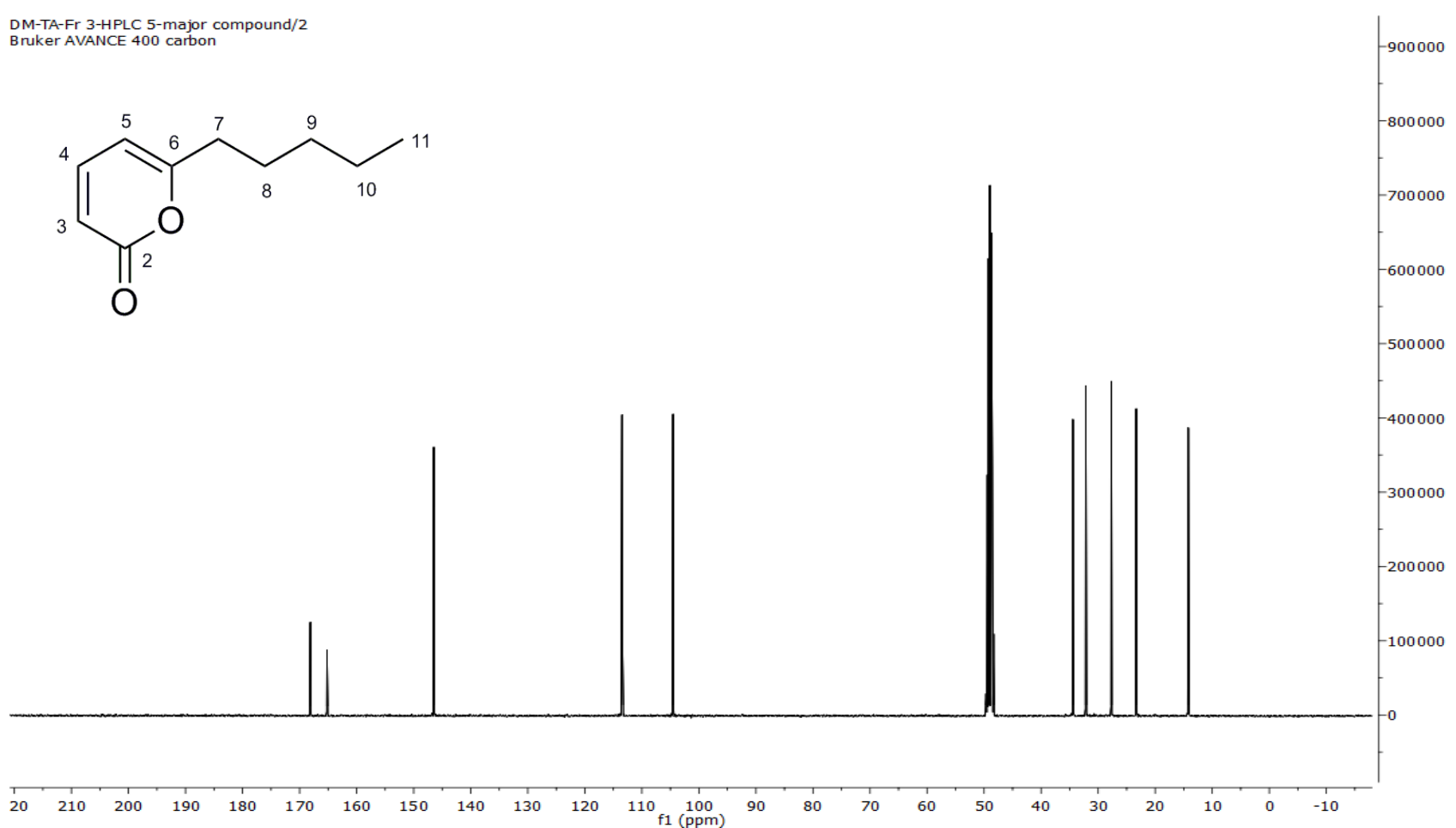

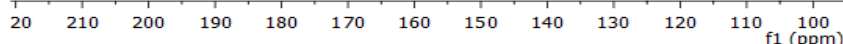




\section{${ }^{1} \mathrm{H}-\mathrm{NMR}\left(\mathrm{CD}_{3} \mathrm{OD}, 400 \mathrm{MHz}\right)$ spectrum of $\mathbf{5 . 1 2}$}

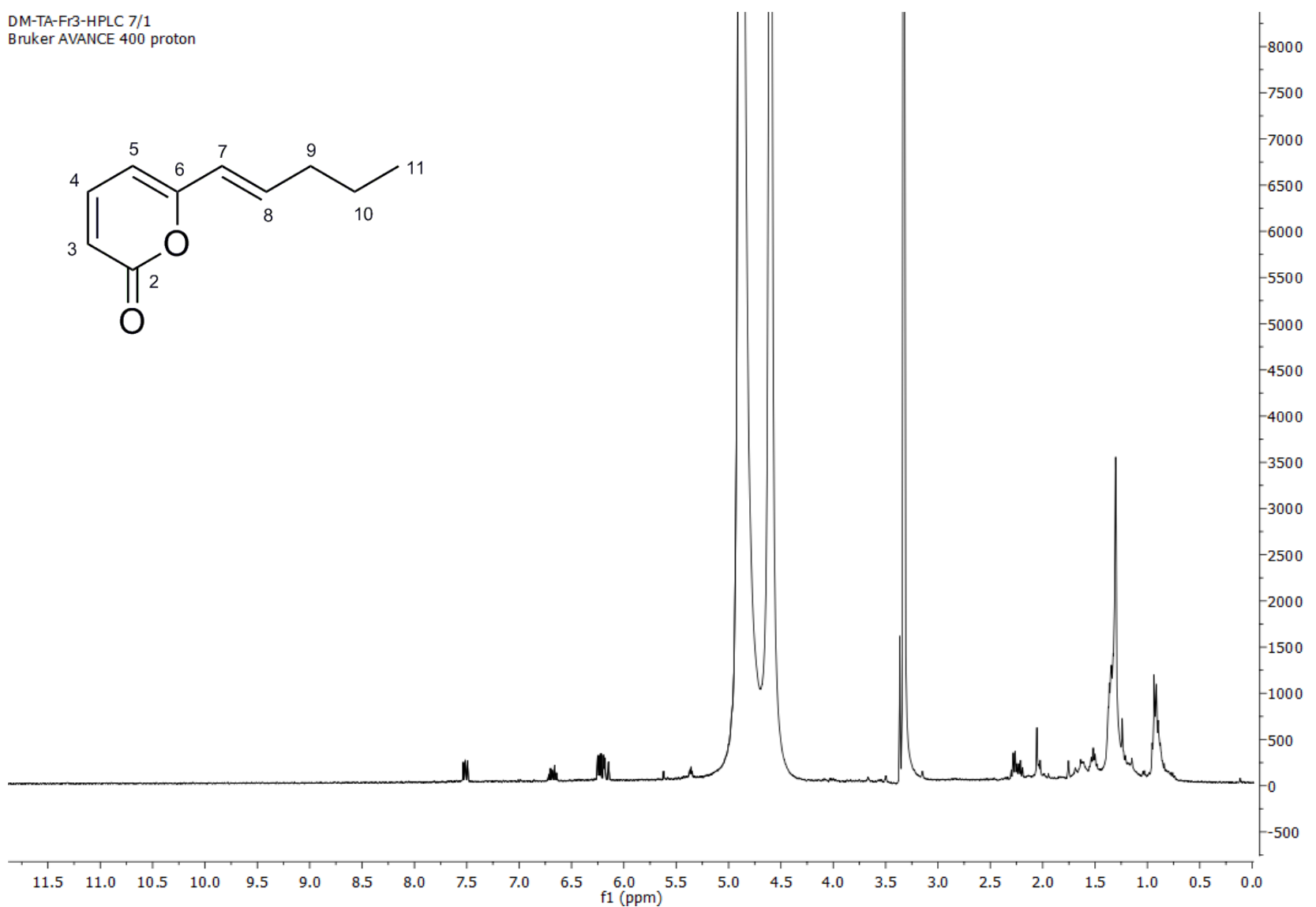


APPENDIX II - Characterization of a $W$. sebi Antigenic Cellulase 
Indirect ELISA were performed as a preliminary means of assessing the reactivity between crude extracellular extracts of the six strains of $W$. sebi tested and the human polyclonal antibodies in the sera from 84 atopic patients (Provost et al. 2013). Of these, 66 sera produced adequate response for subsequent immunoblotting to visualize proteins from culture extracts from each $W$. sebi strain. Based on these data, a $\sim 47 \mathrm{kDa}$ protein was selected for further study as it was present in all strains tested and gave a strong immunoblot response. Additionally, this protein was observed in the extracellular protein extracts and arthrospores of all six $W$. sebi strains studied. Rabbit polyclonal antibodies produced to the target protein responded to the purified target protein as well as with the intracellular proteins and spore protein extracts on Western immunoblots and with a capture ELISA. This confirmed the antigenicity of the target protein in rabbits and humans but most importantly, confirmed the antigens presence in large amounts within the $W$. sebi arthrospores. The $47 \mathrm{kDa}$ protein had an isoelectric point (pI) of 4.9 and was glycosylated. The de-glycosylation procedure revealed that the extent of glycosylation was $\sim 5 \%$ by weight (Desroches et al. 2014).

The semi-purified protein sample was separated by two dimensional SDS-PAGE and visualized by western blot with enhanced anti- $W$. sebi rabbit polyclonal antibodies. Proteins were applied to two IPG strips with a pH range of 4-7 $(7 \mathrm{~cm})$ and left overnight to rehydrate. The IPG strips were focused for 25, $000 \mathrm{~V}$ hours on a Protean IEF cell and loaded onto two SDS-PAGE gels for separation in the second dimension. Proteins were transferred by immuno-blot to a PVDF membrane and stained with $\operatorname{IgG}$ specific antibodies to confirm the presence of the targeted antigenic protein on the first gel. The second gel was silver stained and the corresponding protein was excised, digested with trypsin in-gel and analyzed by nano liquid chromatographic tandem 
mass spectrometry (nLC-ESI-MSMS; table A.1). A Mascot database search performed on the peptide MSMS spectra identifying a cellulase (gi|388581511) from $W$. sebi CBS 633.66. When using a Mascot score greater than 30, seven peptides were observed accounting for $24 \%$ sequence coverage of the full length protein. When setting the Mascot score to 20, the sequence coverage increased to $35 \%$ due to the addition of amino acids $171-213$. This is most likely due to the proline at position 185 inducing conformational restriction in the peptide chain located directly after lysine 184 , the only trypsin cleavage site between these amino acids. The experimentally determined pI of 4.9 compared favorably to the predicted pI of 4.97. Based on analysis with SignalP 4.1, the antigenic cellulase contained a 23 amino acid signal peptide indicating this is an extracellular protein (figure A.1).

The full sequence of $W$. sebi CBS 633.66 was instrumental for the identification of the target antigen as a cellulase (Padamsee et al. 2012). Peptide spectra were additionally verified by manual de novo sequencing. The prevalence of patient sera that respond to fungal allergens has generally correlated with the frequency of the species found in damp buildings in the USA and Canada(Andersen et al. 2011; Miller et al. 2008). P. chrysogenum (P. rubens), the most common species of Penicillium on damp building materials, just over $50 \%$ of the patient sera in from the Miller lab reacted to the allergen (Wilson et al. 2009). Similar results were observed for $C$. globosum, where approximately $50 \%$ of the sera tested responded to the antigenic chitosanase (Provost et al. 2013). Approximately 20\% responded to A. versicolor (Liang et al. 2011) and approximately $10 \%$ for the $S$. chartarum sensu latto antigen (Xu et al. 2007). For W. sebi, 36\% of the sera tested were found to react demonstrating how common this xerophilic species is 
within damp buildings in North America. The $47 \mathrm{kDa}$ excreted cellulase is present in high concentrations in athrospores and is antigenic in humans and rabbits (Desroche et al. 2014).

\section{Protein Identification}

Proteins were separated by two-dimensional gel electrophoresis, digested with trypsin and identified according to Twine et al. (2005). Briefly, extracted proteins from the culture filtrate were separated using linear $\mathrm{pH}$ 4-7, $7 \mathrm{~cm},($ Biorad) immobilized $\mathrm{pH}$ gradient (IPG) strips. $60 \mu \mathrm{g}$ of protein extract was diluted with a solubilization buffer consisting of 4\% CHAPS, 2\% SB3-10, 0.5\% [v/v] pH 3-10 Biolytes (Biorad) and 0.003\% [v/v] Orange G. Proteins were loaded onto IPG strips and allowed to rehydrate overnight. Isoelectric focusing was achieved using a Protein IEF Cell (Biorad) using the following conditions: 200V for 30 minutes, $500 \mathrm{~V}$ for 30 minutes, 2 hour ramp to $6500 \mathrm{~V}$ and 3 hours of focusing at $6500 \mathrm{~V}$ for a total of 20000 to $25000 \mathrm{~V}$ hours. IPG strips were equilibrated for approximately 30 minutes in equilibration buffer consisting of 2\% SDS, $50 \mathrm{mM}$ Tris/HCl pH 8.8, $6 \mathrm{M}$ urea, 30\% glycerol and 1\% DTT followed by the same solution replacing DTT with 4\% iodoacetamide. Proteins were separated in the second dimension on $10 \%$ polyacrylamide gels $(8 \times 10 \mathrm{~cm})$ using a PowerPac $1000($ Biorad) at $150-200 \mathrm{~V}$ for 45 minutes. Gels were fixed with 50\% ethanol, 5\% acetic acid and subsequently stained with silver nitrate to visualize proteins. Protein spots of interest were manually excised from the silver stained gel, de-stained with $15 \mathrm{mM}$ potassium hexacyanoferrate and $50 \mathrm{mM}$ sodium thiosulfate.

Each spot was individually digested with $20 \mathrm{ng} \mu \mathrm{L}^{-1}$ trypsin (Promega) in $50 \mathrm{mM}$ ammonium biocarbonate at $37^{\circ} \mathrm{C}$ overnight. Peptides were extracted from gel spots with $5 \%$ acetonitrile $(\mathrm{ACN})$ in $1 \%$ acetic acid. Peptides were separated by nano-liquid chromatography using a 
"CapLC" capillary chromatography system (Waters) and analyzed by tandem mass spectrometry using a QTOF Ultima hybrid quadrupole time-of-flight mass spectrometer (Waters). Tryptically digested peptides were injected on a reverse phase PepMap $\mathrm{C}_{18}$ nano-column $(75 \mu \mathrm{m} \times 150 \mathrm{~mm})$ (Dionex) and resolved by a linear gradient programmed from 5-75\% solvent B (ACN, $0.12 \%$ formic acid) over 30 minutes at a flow rate of $\sim 350 \mathrm{~nL} \mathrm{~min}^{-1}$. Peptide tandem MS spectra were obtained on the most abundant doubly, triply and quadruply charged ions. Mascot Daemon (Matrix Science) was utilized to search the genome strain Wallemia sebi CBS633.66 and the antigenic protein was identified based on matching peptide sequences derived from the MS/MS spectra. MS/MS spectra of peptides were accessed based on a Mascot score greater than 30 and by manual de novo sequencing. 
Table A.1: Sequence fragments from the nLC-ESI-MS/MS analyses of the $47 \mathrm{kDa} W$. sebi antigenic cellulase (gi|388581511).

\begin{tabular}{llllll}
\hline Observed $(\mathrm{m} / \mathrm{z})$ & MR $($ expt.) & MR (calc.) & Error $(\mathrm{Da})$ & Peptide & Sequence \\
\hline $434.2438\left(2^{+}\right)$ & 866.4730 & 866.4722 & 0.0008 & $90-97$ & K.NAGLTHVR.I \\
$1123.5829\left(2^{+}\right)$ & 2245.1512 & 2245.1532 & -0.0019 & $98-117$ & R.IPVGFWAIETQGEPYIVGNR.L \\
$1103.5541\left(2^{+}\right)$ & 2205.0935 & 2205.0926 & 0.0009 & $135-155$ & K.VWIDLHGAPGSQNGLDNSGLR.T \\
$920.9195\left(2^{+}\right)$ & 1839.8244 & 1839.8248 & -0.0004 & $156-170$ & R.TNNVQWHTDQNNVDR.S \\
$819.8492\left(2^{+}\right)$ & 1637.6838 & 1637.7256 & -0.0418 & $353-366$ & R.QMWNTQVDAFEGGR.G \\
$567.7496\left(2^{+}\right)$ & 1133.4845 & 1133.5334 & -0.0489 & $367-374$ & R.GYFFWTWK.N \\
$684.7692\left(2^{+}\right)$ & 1367.5238 & 1367.5742 & -0.0503 & $375-385$ & K.NEEAADWSYQR.L \\
\hline
\end{tabular}

$\begin{array}{llllll}\text { MKFNILALAA } & \text { AVAASPLQRR } & \text { YGDLIRGVNI } & \text { GGLFVLEPWI } & \text { TPSVFDQTGN } & 50 \\ \text { PAIVDEWTFG } & \text { QYQDHAQAES } & \text { AINSHLETFF } & \text { TYDDFQQIKN } & \text { AGLTHVRIPV } & 100 \\ \text { GFWAIETQGE } & \text { PYIVGNRLNK } & \text { LKEVVRWCRD } & \text { IGLKVWIDLH } & \text { GAPGSQNGLD } & 150 \\ \text { NSGLRTNNVQ } & \text { WHTDQNNVDR } & \text { SLSYIQTLTD } & \text { EFTKPEYGAI } & \text { VEAIELLNEP } & 200 \\ \text { QSATHPEMLG } & \text { TLKSFYQNGY } & \text { GIVSQKAATA } & \text { IHDGFLDVNQ } & \text { WNDFLTSPQE } & 250 \\ \text { NVYLDTHKYQ } & \text { VFSDQQLQSS } & \text { DEQRTGAICQ } & \text { FKDKFAEHTA } & \text { NQHWVITGEW } & 300 \\ \text { SLATTDCARY } & \text { LNGRGIGARY } & \text { DGSYSGSSYV } & \text { GSCQGKTGDG } & \text { SDWSEEYKNQ } & 350 \\ \text { LRQMWNTQVD } & \text { AFEGGRGYFF } & \text { WTWKNEEAAD } & \text { WSYQRLLQLG } & \text { IIPQDPNSYQ } & 400 \\ \text { HGICG } & & & & & 405\end{array}$

Figure A.2: Amino acid sequence of the extracellular W. sebi antigenic cellulase isolated from UAMH7897. Underlined is the 23 amino acid signal peptide and sequenced peptides are indicated in bold when utilizing a Mascot score greater than 30. 